\section{U.S. Department of Commerce}

National E
of Standar INST. OF STAND \& TECH A $1306 \quad 173796$

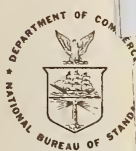

NBS

PUBLICATIONS
NBS Spectal Publícation 260

NBS Standard Reference Materials Catalog 1981-83 Edition

Four 1906 cast irons (right), and nearly 1,000 SRM's of today

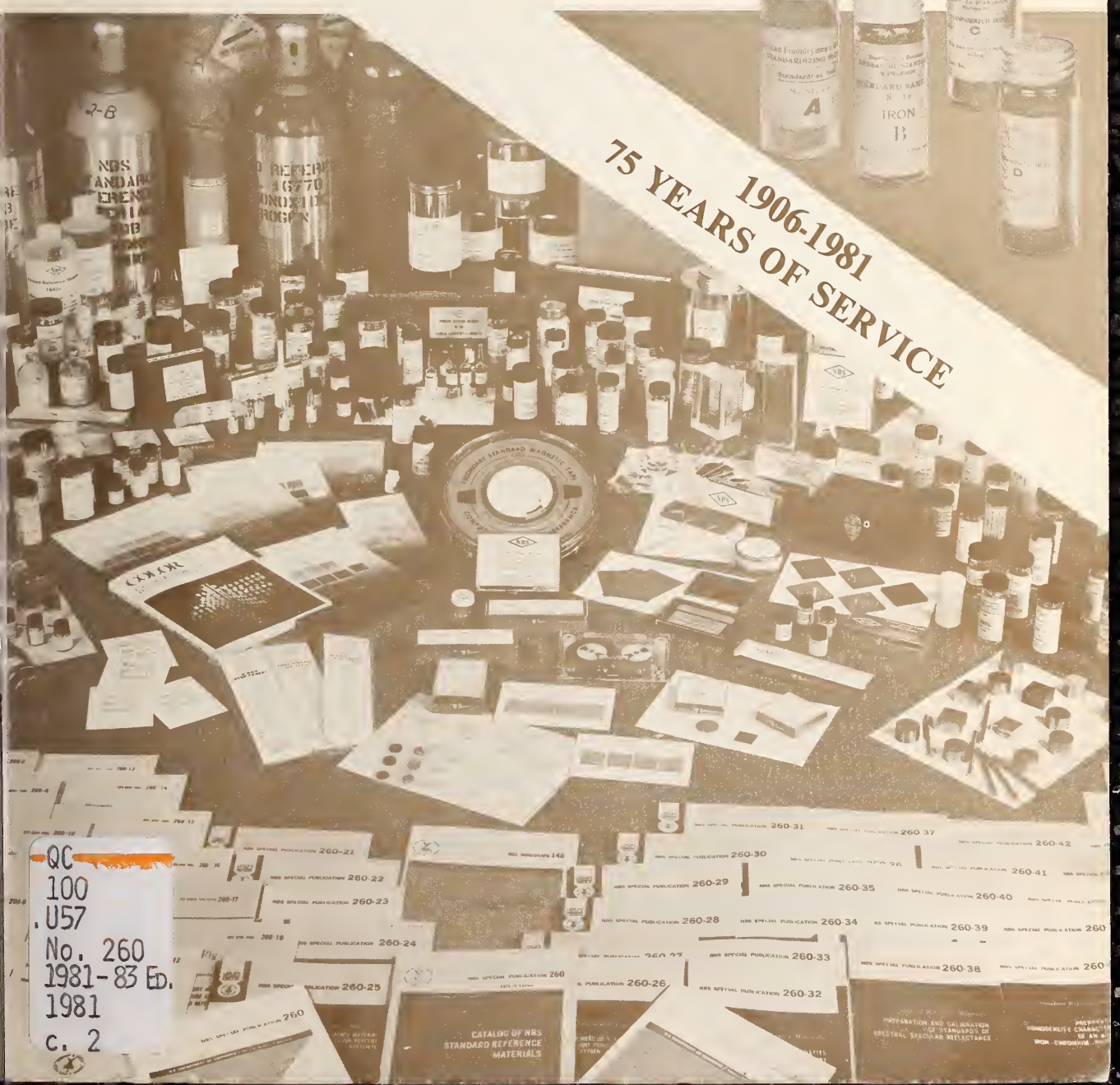




\section{NATIONAL BUREAU OF STANDARDS}

The National Bureau of Standards' was established by an act of Congress on March 3, 1901. The Bureau's overall goal is to strengthen and advance the Nation's science and technology and facilitate their effective application for public benefit. To this end, the Bureau conducts rescarch and provides: (1) a basis for the Nation's physical measurement system, (2) scientific and technological services for industry and government, (3) a technical basis for equity in trade, and (4) technical services to promote public safety. The Bureau's technical work is performed by the National Measurement Laboratory, the National Engineering Laboratory, and the Institute for Computer Sciences and Technology.

THE NATIONAL MEASUREMENT LABORATORY provides the national system of physical and chemical and materials measurement; coordinates the system with measurement systems of other nations and furnishes essential services leading to accurate and uniform physical and chemical measurement throughout the Nation's scientific community, industry, and commerce; conducts materials research leading to improved methods of measurement, standards, and data on the properties of materials needed by industry, commerce, educational institutions, and Government; provides advisory and research services to other Government agencies; develops, produces, and distributes Standard Reference Materials; and provides calibration services. The Laboratory consists of the following centers:

Absolute Physical Quantities ${ }^{2}$ - Radiation Research - Thermodynamics and Molecular Science - Analytical Chemistry - Materials Science.

THE NATIONAL ENGINEERING LABORATORY provides technology and technical services to the public and private sectors to address national needs and to solve national problems; conducts research in engineering and applied science in support of these efforts; builds and maintains competence in the necessary disciplines required to carry out this research and technical service; develops engineering data and measurement capabilities; provides engineering measurement traceability services; develops test methods and proposes engineering standards and code changes; develops and proposes new engineering practices; and develops and improves mechanisms to transfer results of its research to the ultimate user. The Laboratory consists of the following centers:

Applied Mathematics - Electronics and Electrical Engineering ${ }^{2}$ - Mechanical

Engineering and Process Technology $y^{2}$ - Building Technology - Fire Research -

Consumer Product Technology - Field Methods.

THE INSTITUTE FOR COMPUTER SCIENCES AND TECHNOLOGY conducts research and provides scientific and technical services to aid Federal agencies in the selection, acquisition, application, and use of computer technology to improve effectiveness and economy in Government operations in accordance with Public Law 89-306 (40 U.S.C. 759), relevant Executive Orders, and other directives; carries out this mission by managing the Federal Information Processing Standards Program, developing Federal ADP standards guidelines, and managing Federal participation in ADP voluntary standardization activities; provides scientific and technological advisory services and assistance to Federal agencies; and provides the technical foundation for computer-related policies of the Federal Government. The Institute consists of the following centers:

Programming Science and Technology-Computer Systems Engineering.

'Headquarters and Laboratories at Gaithersburg, MD, unless otherwise noted; mailing address Washington, DC 20234.

${ }^{2}$ Some divisions within the center are located at Boulder, CO 80303. 


\section{NBS Special Publication 260 \\ NBS Standard \\ Reference Materials \\ Catalog \\ 1981-83 Edition}

Office of Standard Reference Materials

National Measurement Laboratory

National Bureau of Standards

Washington, DC 20234

CAUTION: The values given in the following sections are listed primarily as a guide to purchase. The values shown are nominal and may differ from those shown on the certificates. Space limitations have required that some values be omitted. For these reasons, the certificates issued with the standards should always be consulted to obtain the certified values.

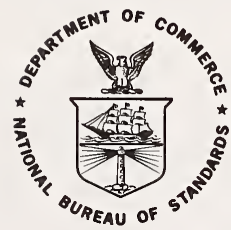

U.S. DEPARTMENT OF COMMERCE

Malcolm Baldrige, Secretary

National Bureau of Standards

Ernest Ambler, Director

Issued November 1981 
National Bureau of Standards Special Publication 260

Supersedes NBS Spec. Publ. 260-1979-80 Edition

Nat. Bur. Stand. (U.S.), Spec. Publ. 260-1981-83 catalog, 122 pages (Nov. 1981)

CODEN: XNBSAV

U.S. GOVERNMENT PRINTING OFFICE WASHINGTON: 1981

For sale by the Superintendent of Documents, U.S. Government Printing Office, Washington, DC 20402 Price

(Add 25 percent for other than U.S. mailing). 


\section{OTHER NBS PUBLICATIONS IN THIS SERIES}

Catalog of NBS Standard Reference Materials (1979-80 edition), R. W. Seward, ed., NBS Spec. Publ. 260 (April 1979) \$3.00* SN003-00302048-6

Michaelis, R. E., and Wyman, L. L., Standard Reference Materials: Preparation of White Cast Iron Spectrochemical Standards, NBS Misc. Publ. 260-1 (June 1964). COM74-11061**

Michaelis, R. E., Wyman, L. L., and Flitsch, R., Standard Reference Materials: Preparation of NBS Copper-Base Spectrochemical Standards, NBS Misc. Publ. 260-2 (October 1964). COM74$11063 * *$

Michaelis, R. E., Yakowitz, H., and Moore, G. A., Standard Reference Materials: Metallographic Characterization of an NBS Spectrometric LowAlloy Steel Standard, NBS Misc. Publ. 260-3 (October 1964). COM74-1 1060**

Hague, J. L., Mears, T. W., and Michaelis, R. E., Standard Reference Materials: Sources of Information, NBS Misc. Publ. 260-4 (February 1965). COM74-11059

Alvarez, R., and Flitsch, R., Standard Reference Materials: Accuracy of Solution X-Ray Spectrometric Analysis of Copper-Base Alloys, NBS Misc. Publ. 260-5 (March 1965). PBl68068**

Shultz, J. I., Standard Reference Materials: Methods for the Chemical Analysis of White Cast Iron Standards, NBS Misc. Publ. 260-6 (July 1975). COM74-1 1068**

Bell, R. K., Standard Reference Materials: Methods for the Chemical Analysis of NBS Copper-Base Spectrochemical Standards, NBS Misc. Publ. 260-7 (October 1965). COM74$11067^{* *}$

Richmond, M. S., Standard Reference Materials: Analysis of Uranium Concentrates at the National Bureau of Standards, NBS Misc. Publ. 260-8 (December 1965). COM74-11066**

Anspach, S. C., Cavallo, L. M., Garfinkel, S. B., Hutchinson, J. M. R., and Smith, C. N., Standard Reference Materials: Half Lives of Materials Used in the Preparation of Standard Reference Materials of Nineteen Radioactive Nuclides Issued by the National Bureau of Standards, NBS Misc. Publ. 260-9 (November 1965). COM74-11065**
Yakowitz, H., Vieth, D. L., Heinrich, K. F. J., and Michaelis, R. E., Standard Reference Materials: Homogeneity Characterization on NBS Spectrometric Standards II: Cartridge Brass and LowAlloy Steel, NBS Misc. Publ. 260-10 (December 1965). COM74-11064**

Napolitano, A., and Hawkins, E. G., Standard Reference Materials: Viscosity of Standard Lead-Silica Glass, NBS Misc. Publ. 260-11 (November 1966). NBS Misc. Publ. 260-11**

Yakowitz, H., Vieth, D. L., and Michaelis, R. E., Standard Reference Materials: Homogeneity Characterization of NBS Spectrometric Standards III: White Cast Iron and Stainless Steel Powder Compact, NBS Misc. Publ. 260-12 (September 1966). NBS Misc. Publ. 260-12**

Spijkerman, J. L., Snediker, D. K., Ruegg, F. C., and DeVoe, J. R., Standard Reference Materials: Mossbauer Spectroscopy Standard for the Chemical Shift of Iron Compounds, NBS Misc. Publ. 260-13 (July 1967). NBS Misc. Publ. 260-13**

Menis, O., and Sterling, J. T., Standard Reference Materials: Determination of Oxygen in Ferrous Materials - SRM 1090, 1091, and 1092, NBS Misc. Publ. 260-14 (September 1966). NBS Misc. Publ. 260-14**

Passaglia, E., and Shouse, P. J. Standard Reference Materials: Recommended Method of Use of Standard Light-Sensitive Paper for Calibrating Carbon Arcs Used in Testing Textiles for Colorfastness to Light, NBS Misc. Publ. 260-15 (June 1967). (Replaced by NBS Spec. Publ. 260-41.)

Yakowitz, H., Michaelis, R. E., and Vieth, D. L., Standard Reference Materials: Homogeneity Characterization of NBS Spectrometric Standards IV: Preparation and Microprobe Characterization of W-20\% MO Alloy Fabricated by Powder Metallurgical Methods, NBS Spec. Publ. 260-16 (January 1969). COM74-1 1062**

Catanzaro, E. J., Champion, C. E., Garner, E. L., Marinenko, G., Sappenfield, K. M., and Shields, W. R. Standard Reference Materials: Boric Acid; Isotopic and Assay Standard Reference Materials, NBS Spec. Publ. 260-17 (February 1970). Out of Print 
Geller, S. B., Mantek, P.A., and Cleveland, N. G., Standard Reference Materials: Calibration of NBS Secondary Standard Magnetic Tape (Computer Amplitude Reference) Using the Reference Tape Amplitude Measurement "Process A, "NBS Spec. Publ. 260-18 (November 1969). (See NBS Spec. Publ. 260-29.)

Paule, R. C., and Mandel, J., Standard Reference Materials: Analysis of Interlaboratory Measurements on the Vapor Pressure of Gold (Certification of Standard Reference Material 745). NBS Spec. Publ. 260-19 (January 1970). PB190071**

Paule, R. C., and Mandel, J., Standard Reference Materials: Analysis of Interlaboratory Measurements on the Vapor Pressures of Cadmium and Silver, NBS Spec. Publ. 260-21 (January 1971). COM74-11359**

Yakowitz, H., Fiori, C. E., and Michaelis, R. E., Standard Reference Materials: Homogeneity Characterization of Fe-3 Si Alloy, NBS Spec. Publ. 260-22 (February 1971). COM74-11357** Napolitano, A., and Hawkins, E. G., Standard Reference Materials: Viscosity of a Standard Borosilicate Glass, NBS Spec. Publ. 260-23 (December 1970). COM71-00157**

Sappenfield, K. M., Marineko, G., and Hague, J. L., Standard Reference Materials: Comparison of Redox Standards, NBS Spec. Publ. 260-24 (January 1972). COM72-50058**

Hicho, G. E., Yakowitz, H., Rasberry, S. D., and Michaelis, R. E., Standard Reference Materials: A Standard Reference Material Containing Nominally Four Percent Austenite, NBS Spec. Publ. 260-25 (February 1971). COM74-11356**

Martin, J. F., Standard Reference Materials: National Bureau of Standards-US Steel Corportion Joint Program for Determining Oxygen and Nitrogen in Steel, NBS Spec. Publ. 260-26 (February 1971). 85 cents* SN003-003-00786-2

Garner, E. L., Machlan, L. A., and Shields, W. R., Standard Reference Materials: Uranium Isotopic Standard Reference materials, NBS Spec. Publ. 260-27 (April 1971). COM74$11358 * *$

Heinrich, K. F. J., Myklebust, R. L., Rasberry, S. D., and Michaelis, R. E., Standard Reference Materials: Preparation and Evaluation of SRM's 481 and 482 Gold-Silver and GoldCopper Alloys for Microanalysis, NBS Spec. Publ. 260-28 (August 1971). COM71-50365**
Geller, S. B., Standard Reference Materials: Calibration of NBS Secondary Standard Magnetic Tape (Computer Amplitude Reference) Using the Reference Tape Amplitude Measurement "Process A-Model 2," NBS Spec. Publ. 260-29 (June 1971). COM71-50282

Gorozhanina, R. S., Freedman, A. Y., and Shaievitch, A. B. (translated by M. C. Selby), Standard Reference Materials: Standard Samples Issued in the USSR (A Translation from the Russian). NBS Spec. Publ. 260-30 (June 1971). COM71-50283**

Hust, J. G., and Sparks, L. L., Standard Reference Materials: Thermal Conductivity of Electrolytic Iron SRM 734 from 4 to 300 K, NBS Spec. Publ. 260-31 (November 1971). COM71-50563**

Mavrodineanu, R., and Lazar, J. W., Standard Reference Materials: Standard Quartz Cuvettes, for High Accuracy Spectrophotometry, NBS Spec. Publ. 260-32 (December 1973). 55 cents* SN003-003-01213-1

Wagner, H. L., Standard Reference Materials: Comparison of Original and Supplemental SRM 705, Narrow Molecular Weight Distribution Polystyrene, NBS Spec. Publ. 260-33 (May 1972). COM72-50526**

Sparks, L. L., and Hust, J. G., Standard Reference Materials: Thermoelectric Voltage, NBS Spec. Publ. 260-34, (April 1972). COM72-50371**

Sparks, L. L., and Hust, J. G., Standard Reference Materials: Thermal Conductivity of Austenitic Stainless Steel, SRM 735 from 5 to $280 \mathrm{~K}$, NBS Spec. Publ. 260-35 (April 1972.) 35 cents* COM72-50368**

Cali, J. P., Mandel, J., Moore, L. J., and Young, D. S., Standard Reference Materials: A Referee Method for the Determination of Calcium in Serum, NBS SRM 915, NBS Spec. Publ. 260-36 (May 1972). COM72-50527**

Shultz, J. I. Bell., R. K. Rains, T. C., and Menis, O., Standard Reference Materials: Methods of Analysis of NBS Clay Standards, NBS Spec. Publ. 260-37 (June 1972). COM72-50692**

Richmond, J. C., and Hsia, J. J., Standard Reference Materials: Preparation and Calibration of Standards of Spectral Specular Reflectance, NBS Spec. Publ. 260-38 (May 1972). COM7250528**

Clark, A. F., Denson, V.A., Hust, J. G., and Powell, R. L., Standard Reference Materials The Eddy Current Decay Method for Resistivity Characterization of High-Purity Metals, NBS Spec. Publ. 260-39 (May 1972). COM72-50529** 
McAdie, H. G., Garn, P. D., and Menis, O., Standard Reference Materials: Selection of Thermal Analysis Temperature Standards Through a Cooperative Study (SRM 758, 759, 760), NBS Spec. Publ. 260-40 (August 1972.) COM-72 $50776^{* *}$

Wood, L. A., and Shouse, P. J., Standard Reference Materials: Use of Standard LightSinsitive Paper for Calibrating Carbon Arcs Used in Testing Textiles for Colorfastness to Light, NBS Spec. Publ. 260-4l (August 1972) COM72-50775**

Wagner, H. L., and Verdier, P. H., eds., Standard Reference Materials: The Characterization of Linear Polyethylene, SRM 1475, NBS Spec. Publ. 260-42 (September 1972). COM7250944**

Yakowitz, H., Ruff, A. W., and Michaelis, R. E., Standard Reference Materials: Preparation and Homogeneity Characterization of an Austenitic Iron-Chromium-Nickel Alloy, NBS Spec. Publ. 260-43 (November 1972). COM73-50760**

Schooley, J. F., Soulen, R. J., Jr., and Evans, G. A., Jr., Standard Reference Materials: Preparation and Use of Superconductive Fixed Point Devices, SRM 767, NBS Spec. Publ. 260-44 (December 1972). COM73-50037**

Greifer, B., Maienthal, E. J., Rains, T. C., and Rasberry, S. D., Standard Reference Materials: Powdered Lead-Based Paint, SRM 1579, NBS Spec. Publ. 260-45 (March 1973). COM7350226**

Hust, J. G., and Giarratano, P. J., Standard Reference Materials: Thermal Conductivity and Electrical Resistivity Standard Reference Materials: Austenitic Stainless Steel, SRM's 735 and 798, from 4 to $1200 \mathrm{~K}$, NBS Spec. Publ. 260-46 (March 1975). SN003-003-01278-5

Hust, J. G., Standard Reference Materials: Electrical Resistivity of Electrolytic Iron, SRM 797, and Austenitic Stainless Steel, SRM 798, from 5 to $280 \mathrm{~K}$, NBS Spec. Publ. 260-47 (February 1974). COM74-50176**

Mangum, B. W., and Wise, J. A., Standard Reference Materials: Description and Use of Precision Thermometers for the Clinical Laboratory, SRM 933 and SRM 934, NBS Spec. Publ. 260-48 (May 1974). 60 cents* SN003-003-01278-5

Carpenter, B. S., and Reimer, G. M., Standard Reference Materials: Calibrated Glass Standards for Fission Track Use, NBS Spec. Publ. 260-49 (November 1974). SN003-003-01344-7
Hust, J. G., and Giarratano, P. J., Standard Reference Materials: Thermal Conductivity and Electrical Resistivity Standard Reference Materials: Electrolytic Iron, SRM's 734 and 797 from 4 to 1000 K, NBS Spec. Publ. 260-50 (June 1975). 1.00 * SN003-003-01425-7

Mavrodineanu, R., and Baldwin, J. R., Standard Reference Materials: Glass Filters As a Standard Reference Material for Spectrophotometry; Selection; Preparation; Certification; Use-SRM 930, NBS Spec. Publ. 260-51 (November 1975). $\$ 1.90 *$ SN003-003-01481-8

Hust, J. G., and Giarratano, P. J., Standard Reference Materials: Thermal Conductivity and Electrical Resistivity Standard Reference Materials 730 and 799 , from 4 to $3000 \mathrm{~K}$, NBS Spec. Publ. 260-52 (September 1975). \$1.05* SN003-00301464-8

Durst, R. A., Standard Reference Materials: Standardization of $\mathrm{pH}$ Measurements, NBS Spec. Publ. 260-53 (December 1975, Revised). \$1.05* SN003-003-01551-2

Burke, R. W., and Mavrodineanu, R., Standard Reference Materials: Certification and Use of Acidic Potassium Dichromate Solutions as an Ultraviolet Absorbance Standard, NBS Spec. Publ. 260-54 (August 1977). \$3.00* SN003-00301828-7

Ditmars, D. A., Cezairliyan, A., Ishihara, S., and Douglas, T. B., Standard Reference Materials: Enthalpy and Heat Capacity; Molybdenum SRM 781, From 273 to 2800 K, NBS Spec. Publ. 260-55 (September 1977). \$2.20* SN00300301836-8

Powell, R. L., Sparks, L. L., and Hust, J. G., Standard Reference Materials: Standard Thermocouple Material, Pt.-67:SRM1967, NBS Spec. Publ. 260-56 (February 1978). \$2.20* SN003-003-018864

Cali, J. P. and Plebanski, T., Guide to United States Reference Materials, NBS Spec. Publ. 260-57 (February 1978). \$2.20* SN003-00301883-0

Barnes, J. D. and Martin, G. M., Standard Reference Materials: Polyester Film for Oxygen Gas Transmission Measurements. SRM 1470, NBS Spec. Publ. 260-58 (in press).

Chang, T. and Kahn, A. H., Standard Reference Materials: Electron Paramagnetic Resonance Intensity Standard: SRM 2601, NBS Spec. Publ. 260-59 (August 1978). \$2.30* SN-003003-01975-5 
Velapoldi, R. A., Paule, R. C., Schaffer, R., Mandel, J., and Moody, J. R., Standard Reference Materials: A Reference Method for the Determination of Sodium in Serum, NBS Spec. Publ. 260-60 (August 1978). \$3.00* SN003-003 01978-0

Verdier, P. H., and Wagner. H. L., Standard Reference Materials: The Characterization of Linear Polyethylene (SRM 1482, 1483, 1484), NBS Spec. Publ. 260-61 (December 1978). \$1.70* SN003-003-02006-1

Soulen, R. J., and Dove, R. B., Standard Reference Materials: Temperature Reference Standard for Use Below $0.5 \mathrm{~K}$ (SRM 768). NBS Spec. Publ. 260-62 (April 1979. \$2.30* SN003-00302047-8

Velapolid, R. A., Paule, R. C., Schaffer, R., Mandel, J., Machlan, L. A., and Gramlich, J. W., Standard Reference Materials: A Reference Method for the Determination of Potassium in Serum, NBS Spec. Publ. 260-63 (May 1979). \$3.75 SN003-003-02068

Velapoldi, R. A., and Mielenz, K. D., Standard Reference Materials: A Fluorescence Standard Reference Material Quinine Sulfate Dihydrate (SRM 936). NBS Spec. Publ. 260-64 (January 1980). \$4.25* SN003-003-02148-2.

Marinenko, R. B., Heinrich, K. F. J., and Ruegg, F. C., Standard Reference Materials: MicroHomogeneity Studies of NBS Standard Reference Materials, NBS Research Materials, and Other Related Samples, NBS Spec. Publ. 260-65 (September 1979). \$3.50* SN003-003-02114-1

Venable, W. H., Jr. and Eckerle, K. L., Standard Reference Materials: Didymium Glass Filters for Calibrating the Wavelength Scale of Spectrophotometers (SRM 2009, 2010, 2013), NBS Spec. Publ. 260-66 (October 1979). \$3.50* SN003-00302127-0

Velapoldi, R. A., Paule, R. C., Schaffer, R., Mandel, J., Murphy, T. J., and Gramlich, J. W., Standard Reference Materials: A Reference Method for the Determination of Chloride in Serum, NBS Spec. Publ. 260-67 (November 1979). \$3.75* SN003003-02136-9

Mavrodineanu, R. and Baldwin, Jr., Standard Reference Materials: Metal-On-Quartz Filters as a Standard Reference Material for Spectrophotometry-SRM 2031, NBS Spec. Publ. 260-68 (April 1980). \$4.25* SN003-003-02167-9
Velapoldi, R. A., Paule, R. C., Schaffer, R., Mandel, J., Machlan, L. A., Garner, E. L., and Rains, T. C., Standard Reference Materials: A Reference Method for the Determination of Lithium in Serum, NBS Spec. Publ. 360-69 (July) 1980). \$4.25* SN003-003-02214-4

Marinenko, R. B., Biancaniello, F., Boyer, P. A., Ruff, A. W., DeRobertis, L., Standard Reference Materials: Preparation and Characterization of an Iron-Chromium-Nickel Alloy for Microanalysis, NBS Spec. Publ. 260-70 (in press).

Reeder, D. J., Coxon, B.,Enagonio, D., Christensen, R. G., Schaffer, R., Howell, B. F., Paule, R. C., Mandel, J., Standard Reference Materials: SRM 900, Antiepilepsy Drug Level Assay Standard, NBS Spec. Publ. 260-72 (in press).

* Send order with remittance to Superintendent of Documents, US Government Printing Office, Washington, DC 20402. Remittance from foreign countries should include an additional one-fourth of the purchase price for postage.

** May be ordered from: National Technical Information Services (NTIS), Springfield, Virginia 22151. 


\section{CONTENTS}

INTRODUCTION

Definitions

Preparation and Availability of Standard

Reference Materials.

Ordering.

Terms

Domestic Shipments

Foreign Shipments

CERTIFIED CHEMICAL COMPOSITION STANDARDS

Steels (chip form)

Plain carbon

Low alloy

High alloy

Stainless

Tool

Steels (granular form)

Steels (solid form)

Ingot iron and low alloy

Special ingot irons and low alloy

Stainless

Specialty

High-temperature alloys

Tool

Steelmaking Alloys

29

Cast Irons (chip form)

Cast Steels, White Cast Irons, Ductile

Irons, and Blast Furnace Irons (solid form)

Nonferrous Alloys (chip form)

Aluminum "Benchmarks"

Copper

Copper "Benchmarks"

Lead

Magnesium

Nickel

Nickel Superalloy, Trace Elements

Nickel oxide

Selenium

Tin

Titanium

Zinc

Zirconium

Nonferrous Alloys (solid form)

Aluminum "Benchmarks"

Copper

Copper "Benchmarks"
Lead

Nickel

Titanium

Zinc

Zirconium

Gases in Metals.

41

High-Purity Metals

41

Microanalytical Standards

42

Primary, Working, and Secondary

Standard Chemicals

Microchemical Standards

Clinical Laboratory Standards...................... 45

Biological Standards

Environmental Standards

Analyzed gases

Analyzed liquids and solids

Permeation tubes

Industrial Hygiene Standards.

Forensic Standards

Metallo-Organic Compounds

Wear Metals in Oil

Fertilizers

Ores

Minerals, Refractories, Glasses, and

Carbides.

Cements

Trace Element Standards

Nuclear Materials

Special nuclear materials

Plutonium assay

Plutonium isotopic

Uranium assay

Uranium isotopic

Neutron density standards

Fission track glass standards

Isotopic Reference Standards.

\section{CERTIFIED PHYSICAL PROPERTY} STANDARDS

Ion Activity Standards

$\mathrm{pH}$ standards

pD standards

Ion selective electrodes

Mechanical and Metrology Standards .......... 62

Scanning Electron Microscope Standards.... 62

Optical Microscope Linewidth-

Measurement Standards

Coating thickness

Glass 
Elasticity

Density

Polymer

Rheology

Heat Standards

Superconductive thermometric fixed point devices

Freezing points

Defining fixed points

Determined reference points

Melting points

Calorimetric

Combustion

Solution

Heat source

Enthalpy and heat capacity

Vapor pressure

Conductivity

Resistivity

Thermal conductivity and electrical resistivity

Thermal expansion

Thermocouple materials

Thermal resistance

Magnetic Standards

Magnetic susceptibility

Magnetic moment

Paramagnetic resonance

Optical Standards

Spectrophotometric

Reflectance

Specular Spectral Reflectance

Directional-Hemispherical Reflectance

Refractive index

Radioactivity Standards

Alpha-particle standards

Beta-particle and gamma-ray gas standards

Alpha-particle, beta-particle, gamma-ray, and electron-capture solution standards

Contemporary standard for carbon- 14 dating laboratories

Environmental standards

Low energy photon sources

Gamma-ray "point-source” standards

Radium gamma-ray solution standards

Radium solution standards for radon analysis

Radioactivity standard reference materials currently not in stock
Metallurgical.

Mössbauer

$\mathrm{X}$-ray Flourescent Emission Target .............. 82

X-ray Diffraction ............................................. 82

Gas Transmission

Permittivity.

Reference Fuels

Resistivity

ENGINEERING TYPE STANDARDS

Standard Rubber and RubberCompounding Materials

Reference Magnetic Tapes

Centerline Drawings, OCR-B

Sizing Standards

Glass spheres for particle size

Turbidimetric and fineness (cement)

Color Standards

Light-Sensitive Papers and Plastic Chips .....

Light-Sensitive Papers

Light-Sensitive Plastic Chips

X-ray and Photographic Standards .............. 86

Surface Flammability Standards .................... $\quad 87$

Smoke Density Chamber Standards.............. $\quad 87$

Tape Adhesion Testing Standard

RESEARCH MATERIALS

Phosphorus

SPECIAL REFERENCE MATERIALS....... Differential Thermal Analysis Standards

STANDARD REFERENCE MATERIALS TO BE DISCONTINUED

OTHER SERVICES OF THE NATIONAL BUREAU OF STANDARDS

Calibration and Related Measurement

Services

Standards Information Service

Standard Reference Data

Technical Information and Publications

GUIDE FOR REQUESTING THE DEVELOPMENT OF STANDARD REFERENCE MATERIALS

CERTIFIED REFERENCE MATERIALS FROM OTHER SOURCES .................... 97

INDICES........................................................ 99

Alphabetical Index ......................................... $\quad 99$

Numerical Index to SRM Certificates........... 106 


\title{
Catalog of Standard Reference Materials - 1981-83
}

\begin{abstract}
This Catalog lists those Standard Reference Materials (SRM's), Research Materials (RM's), and Special Reference Materials (GM's) that are available from the National Bureau of Standards (NBS), and those that are soon to be available. The Catalog describes these materials as to their certified characterization, unit size, and type, as well as providing ordering information. Prices for these materials are listed separately in annual supplements to this Catalog.
\end{abstract}

Key words: Analysis; characterization; composition; properties; Standard Reference Materials; Research Materials; materials.

\section{Introduction}

The National Bureau of Standards issues over 1000 different materials through its Standard Reference Materials Program. These materials are primarily Standard Reference Materials (SRM's) certified for their chemical composition, chemical property, or physical property, but also include Research Materials (RM's) and Special Reference Materials (GM's). All SRM's, RM's, and GM's bear distinguishing names and numbers by which they are permanently identified. Thus, each SRM, RM, or GM bearing a given description is identical (within the required or intended limits) to every other sample bearing the same designation-with the exception of individually certified items, which are further identified by serial number.

The first materials issued by NBS were called Standard Samples and consisted of a group of ores, irons, and steels certified for their chemical composition. Since the mid-1960's these materials have been issued as Standard Reference Materials, and cover a wide range of chemical and physical properties and an equally wide range of measurement interests.

\section{Definitions}

The different terms, SRM, RM, or GM, are used to indicate differences in the types of information supplied and in the purposes for which the material is intended.

Standard Reference Materials have been characterized by the National Bureau of Standards for some chemical or physical property and are issued with a Certificate that gives the results of the charaterization. These results are obtained by one of the three established routes of certification, i.e., measurement of the property using: (1) a previously validated reference method, (2) two or more independent, reliable measurement methods, or (3) an ad hoc network of cooperating laboratories, technically competent and thoroughly knowledgeable with the material being tested. These routes are described in detail in, "The Role of Standard Reference Materials in Measurement Systems," NBS Monograph 148, 54 pages (Jan. 1975). SRM's are defined as being well-characterized and certified materials produced in quantity to improve measurement science. They are prepared and used for three main purposes: to help develop accurate methods of analysis (reference methods); to calibrate measurements systems used to: (a)facilitate the exchange of goods, (b) institute quality control, (c) determine performance characteristics, or (d) measure some property at the limit of the state-of-theart; to assure the long-term adequacy and integrity of quality control processes. In these ways, SRM's help ensure the compatibility and accuracy of measurements in many facets of national lifefrom science and technology to trade and commerce.

Research Materials, unlike SRM's, are not certified. Instead of a Certificate, RM's are issued with a "Report of Investigation," the sole authority of which is the NBS staff member who authored the report. An $\mathrm{RM}$ is intended primarily to further scientific or technical research on that particular material. The principal consideration in issuing an $\mathrm{RM}$ is to provide a homogeneous material so that investigators in different laboratories are assured that they are investigating the same material.

Special Reference Materials differ from both SRM's and RM's in that NBS does not participate in the 
characterization of these materials. GM's are reference materials produced and certified or guaranteed by other government agencies, standards bodies, or other non-profit organizations. When deemed to be in the public interest and when alternate methods of national distribution do not exist, NBS acts as the distributor for such materials. This service is available to all organizations that qualify and have reference materials that would help solve a national measurement problem.

\section{SRM Catalog}

New Catalogs of NBS Standard Reference Materials are published approximately every 3 years, listing new materials available, materials in preparation, and deleting discontinued materials. To keep the Catalog current between editions, annual supplements are published that list: the current prices charged, new materials, discontinued materials (with suggested substitutes), and revised Certificates.

The materials listed in this Catalog are separated into several major groups: Chemical Composition Standards, Physical Property Standards, Engineering Type Standards, Research Materials, and Special Reference Materials. The first three groups include SRM's only and are subdivided into the categories shown in the Table of Contents.

The numerical values given in the Catalog to describe the materials' properties are nominal values only and are to be used only as guides in selecting the materials. They are not to be used in lieu of the Certificate issued with the material.

Note: Some SRM's are not issued with Certificates. These exceptions are noted in both the SRM description and the numerical index.

The numerical index provides the SRM, RM, and GM numbers of all materials listed in the Catalog, together with either the date of the current Certificate or a note to explain the absence of a Certificate.

An alphabetical index is also provided. For the most part, this index lists SRM catagories and the primary constituent or element of an SRM, rather than the names of specific SRM's. RM's and GM's are listed as groups only.

\section{Preparation and Availability of Standard Reference Materials}

New and renewal SRM's are being prepared continually. When completed, prospective users are notified directly of their availability, and the SRM's are described in the next catalog supplement.

In preparing renewal SRM's, the intention is to complete the renewal before the supply of the existing
SRM is exhausted. Frequently, this is not possible and the SRM will be out-of-stock for a time. When this occurs, those ordering the material are so notified and, when feasible, of possible substitutes. (See Ordering below). When the renewal becomes available, customers who have requested either the previous lot or the renewal are promptly notified.

Renewal SRM's will not usually be identical to their predecessors, but will be quite similar especially with regard to the characteristics certified. Generally, the renewal can be used in place of its predecessor. As an example, when the first 0.1 percent carbon Bessemer steel was prepared in 1909 , it was called Standard Sample No. 8. During the following years, a number of renewals, $8 \mathrm{a}, 8 \mathrm{~b}$, etc., were prepared. The current SRM 8j, Bessemer Steel (Simulated), 0.1\% Carbon, represents the 10 th renewal batch of this material. While each of these batches differs somewhat in detailed analysis, all have had the relatively high level of phosphorus, sulfur, and nitrogen, and low alloy metal content characteristic of this type of material.

It is not possible to supply preceding numbers of a renewal series when the stock is exhausted. If little demand exists or an alternate source of supply becomes available for a material, production may be discontinued permanently.

\section{Ordering}

Orders should be addressed to:

$$
\begin{gathered}
\text { Office of Standard Reference Materials } \\
\text { Room B311, Chemistry Building } \\
\text { National Bureau of Standards } \\
\text { Washington, DC 20234 } \\
\text { Telephone (301) 921-2045 }
\end{gathered}
$$

Orders should give the amount (number of units), catalog number, and name of the material requested. For example: 1 each, No. $11 \mathrm{~h}$, Basic-Open-Hearth Steel, 0.2 percent C. The materials described in this Catalog are distributed in the units listed or in multiples thereof only.

Acceptance of an order does not imply acceptance of any provision set forth in the order contrary to the policy, practice, or regulations of the National Bureau of Standards or the U.S. Government.

Orders received for "out-of-stock" materials are cancelled if only out-of-stock items are ordered. On other orders, shipment is made of available materials and out-of-stock items are cancelled. Backorders are not accepted for out-of-stock materials; if a renewal lot of material is available, it will be furnished automatically. 


\section{Terms}

Prices quoted are in U.S. dollars, and are published in the SRM Price List (Supplement to this Catalog). New SRM Price Lists, when issued, are sent to users who have made purchases during the preceeding 12 months and to persons or organizations who have requested them. These prices are subject to revision without notice and orders will be billed for the prices in effect at the time of shipment. No discounts are given on purchases of SRM's, RM's, or GM's.

Remittances of the purchase price need not accompany purchase orders. Payment of invoices is expected within 30 days of receipt of an invoice. Payment on foreign orders may be made by any of the following:

(a) UNESCO coupons.

(b) banker's draft against a U.S.A. bank,

(c) bank to bank transfer to a U.S.A. bank,

(d) letter of credit on a U.S.A. bank, or

(e) by International Money Order.

Pro-forma invoice service will frequently require 6 to 8 weeks to process, and will be furnished only to those requiring such service.

Letters of Credit may be used as advance payment for SRM's. Letters of credit will be accepted from banks in the United States only. Listed below are the only documents that we will furnish:

(1) Six Commercial Invoices

(2) Packing List

(3) Certificate of Origin

(4) Airway Bill (This can only be furnished if material is shipped collect; if shipped prepaid International Air Parcel Post, receipts cannot be furnished.)

If, however, the purchaser requests a different mode of shipment, the shipment will be sent collect. For items shipped collect NBS cannot "prepay and add" such shipping charges to the invoice. Restricted categories such as hydrocarbons, organic sulfur compounds, compressed gases, rubber, rubber compounding materials, radioactive standards, and similar materials are shipped FOB Gaithersburg, MD.

\section{Domestic Shipments}

Shipments of material (except for certain restricted categories) intended for the United States, Mexico, and Canada are normally shipped prepaid (providing that the parcel does not exceed the weight limitations as prescribed by Postal Laws and Regulations).

\section{Foreign Shipments}

The regulations of various nations covering the importation of SRM's, GM's, and RM's differ widely; any attempt to list all possible variations would be impractical. Therefore, where the shipping practices outlined below do not apply, purchasers will be informed of the best method of shipment for their countries.

Most orders will be shipped by prepaid International Air Parcel Post. Exceptions are items in restricted categories and those shipments that exceed parcel post weight limitations. These exceptions will be shipped FOB Gaithersburg, MD, unless an agent (shipping or brokerage firm) located in the United States is required. Where an agent is required, the purchaser will be so notified and will be requested to designate an agent of his/her choice. In this case, the material will be packaged for overseas shipment and will be forwarded to the agent FOB Gaithersburg, MD.

NOTE: Orders and inquiries submitted in English will be processed more rapidly than those requiring translations. 


\title{
National Bureau of Standards
}

\section{Catálogo de Materiales Patrones de Referencia del año 1981-83}

\begin{abstract}
En este catálogo figuran todos los Materiales Patrones de Referencia (SRM's), Materiales de Investigación (RM's) y Materiales Especiales de Referencia (GM's) que pueden ser obtenidos en el National Bureau of Standards (NBS) en la actualidad y aquellos que estarán disponibles en breve plazo. Estos materiales están descritos en el catálogo en la misma forma que en el certificado haciendo referencia al tamaño, clase, y características asi como información para hacer los pedidos. Los precios figuran por separado en un suplemento anual de este catálogo.
\end{abstract}

Palabras Claves: Análisis; certificación; caracterización; composición; propiedades; Materiales de Investigácion (RM's); Materiales Especiales de Referencia (GM's) y Materiales Patrones de Referencia (SRM's).

\section{Introducción}

NBS emite más de 1000 materiales diferentes a través de su programa para SRM's. Estos materiales son principalmente SRM's que han sido certificados por su composición química y propiedades químicas y físcas pero tembién incluye RM's y GM's. Todos los SRM's, RM's y GM's llevan nombres y números que los identifican permanentemente. Cada SRM, RM o GM al cual se la ha dado una descripción determinada es idéntica a todas las otras muestras que llevan la misma designación (dentro de los límites requeridos para su uso) con excepción de aquellas que han sido certificadas individualmente y a las cuales se les asigna un número de serie.

Los primeros materiales emitidos por el N.B.S. fueron llamados "muestras patrones" (standard samples) y consistián en un grupo de minerales, hierros y aceros que habián sido certificados por su composición química, pero a partir del año de 1960 se les disignó con el nombre de Materiales Patrones de Referencia (SRM's) que cubre un campo más amplio de propiedades físicas y químicas y que refleja el interés por las medidas.

\section{Definiciones}

Los términos SRM, RM, y GM son usados para indicar las diferentes clases de información suministrada y el propósito al cual el material ha sido destinado.

Materials Patrones de Referencia: N.B.S. ha caracterizado los SRM's por algunas de sus propiedades físicas y químicas y los emite con un certificado en el cual aparece el resultado de dicha caracterización.
Estos resultados son obtenidos por una de las tres vías de certificación establecidas. Medida de la propiedad usando:

(1) Un método de referencia con validez probada previamente.

(2) Dos o más métodos independientes de medidas dignos de confianza.

(3) Una red de cooperativas de laboratorios tecnicamente competentes y con conocimiento cabal del material comprobado para este específico propósito.

Estas vías han sido descritas detalladamente en " $\mathrm{La}$ Función de los Materiales Patrones de Referencia en el Sistema de Medidas" (The Role of Standard Reference Materials in Measurement Systems, NBS Monograph 148, 54 pages, Jan. 1975). Se ha definido los SRM's como materiales que se han certificado y analizado en cantidades suficientes para ser usados en el mejoramiento de las ciencias de las medidas. Los SRM's han sido preparados y usados con tres principales propósitos: para ayudar a desarrollar métodos de análisis de alta calidad (Metodos de Referencia); para calibrar sistemas de medidas destinados a: (a) facilitar el intercambio de mercancías; (b) implantar control de la calidad, (c) determinar las características representadas y (d) llevar la medida de algunas propiedades al límite de la Obra de Arte; y para asegurar un método de control de la calidad adecuado, duradero e íntegro. En esta forma los SRM's auydan a asegurar la compatibilidad y alta calidad de las medidas en muchos aspectos de la vida nacional desde la ciencia y la tecnología hasta los oficios y el comercio. 
Materiales de Investigación (RM's): A diferencia de los SRM's, éstos no están certificados. En lugar de un certificado, ellos son emitidos con un informe de investigación (Report of Investigation) el cual es responsabilidad del miembro del cuerpo de científicos del NBS que produce el informe. RM's estan destinados principalmente a fomentar la investigación científica y técnica sobre determinado material con la principal concideración de proveer un material homogéneo a los científicos en los diferentes laboratorios, con la seguridad de que están investigando el mismo material.

Los Materiales Especiales de Referencia (GM's) se distinguen de los SRM's y de los RM's en que el N.B.S. no participa en su investigación. Los GM's son materiales de referencia producidos y certificados por otras agencias del gobierno, otros grupos de patrones de referencia o algunas organizaciones no lucrativas. N.B.S. actúa como el distribuidor de dichos materiales cuando considera que es del interés público o cuando no hay otro medio mejor para la distribución nacional. Este servicio esta al alcance de las organizaciones que llenen los requisitos requeridos y tengan materiales de referencia que pudieran ayudar a resolver un problema nacional de medida.

\section{Catálogo de Materiales Patrones de Referencia}

El Programa de los SRM's del N.B.S. publica nuevos catálogos cada 3 años en los cuales se registran los nuevos materiales disponibles, materiales en preparación y materiales que han sido descontinuados.

Para mantener el catálogo al corriente entre ediciones se publica un suplemento con la lista de los precios actuales, nuevos materiales, materiales descontinuados (con algunas sugerencias de sustitutos) y certificados revisados.

Los materiales que figuran en el catálogo han sido clasificados en varios grandes grupos: Patrones de Composición Química, Patrones de Propiedades Físicas, Patrones de Tipo de Ingeniería y Patrones Generales. Los 3 primeros tipos incluyen solamente SRM's y están divididos en diferentes sub-grupos que aparecen en el Indice General.

Los valores numéricos ofrecidos en el catálogo que describen las propiedades de los materiales son valores nominales para ser usados solamente como guías al seleccionar los materiales. Estos valores numéricos no deben ser usados en lugar del certificado emitido con el material.
Nota: Algunos SRM's no son emitidos con certificados. Estas excepciones aparecen en la descripción de SRM's y en el indice numérico.

El indice numérico ofrece números para todos los materiales que aparecen en el catálogo junto con la fecha vigente o en su lugar una nota explicando la ausencia del certificado.

Un índice alfabético es ofrecido donde se enumera los SRM's y el componente o elemento principal en lugar del nombre del SRM's en cuestión. Los SRM's y GM's aparecen en grupos solamente.

\section{Preparación Y Disponibilidad de los Materiales Patrones de Referencia.}

Continuamente se están renovando y preparando nuevos SRM's. Cuando están disponibles se notifica a los interesados y se describen en el próximo suplemento del catálogo.

La renovación de los SRM's tiene como finalidad asegurar la existencia de los materiales antes que se agoten sus reservas. Algunas veces ésto no es posible y algunos materiales están fuera del mercado por un tiempo. Cuando ésto ocurre se le notifica a aquellos que han encargado el material informándoles cuando podrán ser obtenidos o la posibilidad de sustitutos. Cuando el material renovado vuelve a estar disponible se le notifica a los clientes que lo habián solicitado previamente (vea pedidos). Por lo general el material de renovación no será idéntico a su predecesor pero será bastante similar en especial a lo que a sus caracteres certificados se refiere. En general el material renovado puede ser usado en lugar de su predecesor. Por ejemplo cuando el primer acero Bessemer con 0.1 por ciento de Carbono fué preparado en el año 1909 fué designado como "Standard Sample No. 8". Durante los años subsiguientes fueron preparados lotes de renovacion $8 \mathrm{a}, 8 \mathrm{~b}$ etc. En la actualidad está disponible el SRM 8j que representa la décima renovación del acero Bessemer con 0.1 por ciento de Carbono. Si bien cada uno de estos lotes difiere en algo en cuanto al análisis detallado entre uno otro lote, todos tienen el nivel relativamente alto de contenido de fósforo, azufre, nitrógeno y metal de baja aleación que es característica de este material.

Una vez agotadas las existencias no es posible suministrar números precedentes de una serie de renovaciones. Si existe poca demanda o se dispone de otra fuente de suministro de determinado material, la producción puede ser descontinuada en forma permanente. 


\section{Pedidos}

Los pedidos deberán ser hechos a la siguiente dirección.
Office of Standard Reference Materials
Room B311, Chemistry Building
National Bureau of Standards
Washington, D.C. 20234
Teléfono (301) 921-2045

En los pedidos se deberá indicar la cantidad (Número de unidades), el número del catálogo y el nombre del material ordenado. Por ejemplo: una muestra, No. 11h, Basic-open-Hearth Steel, 0.2 por ciento C. Estos materiales son distribuidos solamente en las medidas que figuran en la lista. La aceptación de un pedido no implica la aceptación de ninguna estipulación que vaya en contra de la política, práctica o regulaciones del N.B.S. o del Gobierno de los E.U.

Pedidos que se reciban de materiales "agotados" son cancelados, si se ha pedido solamente materiales agotados. Si el pedido incluye otros materiales disponibles se envían éstos y se cancelan los materiales agotados. No se aceptan pedidos de materiales agotados. Si un lote de material de renovación está desponible será suministrado automaticamente.

Los precios están fijados en moneda de los E.U.y son publicados en la lista de precios de los SRM's (suplemento del catálogo). Cuando las nuevas listas de precios son emitidas se les envían a los clientes que han hecho compras en el curso de los últimos doce meses y a las personas y organizaciones que las soliciten.

Estos precios están sujetos a revisiones sin previa notificación y los pedidos se enviarán con los precios que estén vigentes en el momento del despacho de la mercancía.

Las órdenes de compra no necesitan ir acompañadas del valor de la compra. Se espera que las facturas sean pagadas dentro de los treinta días después de recibidas. El pago de pedidos desde el extrajero se puede hacer mediante cualquiera de las siguientes vías:

a) Cupones de la Unesco

b) Giro bancario contra un banco en los E.U.

c) Transferencia bancaria

d) Carta de Crédito en un banco de los E.U. (ver nota)

e) Giro Internacional.

Suele requirir ocho semanas procesar facturas proforma y serán suministradas solamente a aquellos que requieran tal servicio.

Nota: Se puede emplear cartas de crédito para pagar por adelantado los SRM's. Se aceptarán unica- mente cartas de crédito de bancos en los E.U. A continuación se indican los únicos documentos que serán suministrados.

1) Seis facturas comerciales.

2) Lista de embalaje.

3) Certificado de origen.

4) Factura de embarque aéreo (solamente si el flete se remite a ser pagado). No se suministrará recibo cuando la mercancía es enviada por el Servicio Postal Aereo Internacional (Porte pagado).

\section{Envios Domésticos}

Los envíos de materiales (a excepción de ciertas categorías restringidas) destinados a los E. U. y Canada por lo general son remesados con importe pagado (siempre cuando el paquete no exceda las limitaciones prescritas por las leyes y el reglamento postal). Sin embargo, si el comprador solicita una forma diferente de envío, la mercancía se enviará con flete por pagar. N.B.S. no Paga Por Tales Fletes. Algunas categorías restringidas como hidrocarburos, compuestos orgánicos de azufre, gases comprimidos, materiales para compuestos de goma, muestras radioactivas y materiales semejantes serán enviados desde Gaithersburg, MD. por expreso con flete por pagar.

\section{Envios al extranjero}

Las regulaciones que cubren la importación de SRM's, GM's y RM's de algunos paises difieren grandemente. Hacer una lista de estas diferencias no tiene objeto por lo tanto los compradores deberán nformarse de cual es el mejor método de envío existente en sus paises cuando la siguiente lista de prácticas no es aplicable.

La mayoría de los pedidos con flete pagado serán enviados por Encomienda Postal Internacional Aerea. Son excepciones los artículos en categorías restringidas y aquellos que excedan las limitaciones de peso establecidas por la oficina postal de paquetes. Estas excepciones serán enviadas por expreso con flete por cobrar a menos que un agente (empresa de transporte o corredor) ubicado en los E.U. sea requerido. Si es necesario hacer el envío a través de un agente se notificará al comprador para que escoja al agente que deseé. En este caso el material será embalado para embarque marítimo y despachado por expreso con flete por cobrar a la empresa en las E.U. que ha sido designada como agente.

Nota: Pedidos y consultas presentados en idioma inglés serán tramitados en forma más rápida que aquellos que requieran traducción. 


\title{
National Bureau of Standards (NBS)
}

\section{Catalogue des Materiaux Standard de Reference 1981-83}

\begin{abstract}
Ce catalogue répertorie les matériaux standard de référence (Standard Reference Materials, SRM's), les matériaux de recherches (Research Materials, RM's) et les materiaux speciaux de références (Special Reference Materials, GM's) disponibles au Bureau National des Standards (National Bureau of Standards, NBS) et ceux qui seront bientôt disponibles. Pour chacun de ces matériaux, le catalogue donne les caractéristiques certifiées, la description de l'échantillon (dimensions, forme), et fournit des renseignements commerciaux. Les prix sont portés séparément dans des suppléments annuels à ce catalogue.
\end{abstract}

Mots-clés: Analyse; certification; caractérisation; composition; propriétés; Research Materials; Special Reference Materials; Standard Reference Materials.

\section{Introduction}

Le Bureau National des Standards (National Bureau of Standards, NBS)* délivre plus de 1000 matériaux différents grâce à son programme de Materiaux Standard de Référence (Standard Reference Materials, SRM's)*. Ces matériaux sont principalement des SRM's certifiés pour leur composition chimique, leur propriété chimique ou leur propriété physique, mais comprennent également des matériaux de recherche (Research Materials, RM's)* et des materiaux specials de references (Special References Materials, GM's).* Tous les SRM's, RM's et GM's portent des noms et des numéros distinctifs qui permettent de les identifier en permanence. Ainsi, chaque SRM, $\mathrm{RM}$, ou GM désigné par une description donnée est identique (dans les limites spécifiées ou visées) à tous les autres échantillons de même désignation, à l'exception des articles certifiés isolément, qui sont identifiés en plus par un numéro de série.

* Nota: Ces expressions ou les abreviations correspondentes seront utilisées par la suite dans ce document.

Les premiers matériaux délivrés par le NBS portaient le nom d'échantillon standard (Standard Samples) et comprenaient un groupe de minerais, de fers, et d'aciers, certifiés pour leur composition chimique. Depuis le milieu des années 60 , ces matériaux ont été délivrés sous la forme de "Standard Reference Materials" et couvrent une large gamme de propriétés physiques et chimiques, et une gamme tout aussi large de domaines d'intérêt.

\section{Definitions}

L'emploi des différents termes SRM, RM, ou GM, est destiné á différencier les types d'informations fournies et les utilisations auxquelles est destiné le matériau.

Standard Reference Materials. Ils sont caractérisés par le NBS pour une propriété physique ou chimique donnée et sont délivrés avec un Certificat donnant les résultats de la caractérisation. Ces résultats sont obtenus par l'une des trois procédures de certification, c'est-à-dire de mesure de la propriété, suivantes: (1) par une méthode de référence précédemment reconnue, (2) par deux ou plusieurs méthodes de mesures différentes et fiables, ou (3) par un réseau ad hoc de laboratoires participants, ayant la compétence technique et la connaissance du matériau à caractériser. Ces procédures sont décrites en détail dans "The Role of Standard Reference Material Measurement Systems" NBS Special Publication 148 (1975). Les SRM's sont définis comme des matériaux caractérisés avec soin et certifiés, produits en quantité, afin d'améliorer la science des mesures. Ils sont élborés et utilizés pour répondre à trois objectifs principaux: Aider à développer des méthodes d'analyses de justesse (méthodes de référence); Etalonner des appareillages de mesures pour: (a) faciliter les échanges de produits, (b) instituer les contrôles de qualité, (c) évaluer des performances, ou (d) mesurer une propriété donnée au mieux des connaissances actuelles; Assurer à long terme la validité et l'intégrité des processus de contrôle de qualité. Sous tous ces aspects, les SRM's permettent d'assurer la cóhérence et la justesse des mesures, dans de nombreuses facettes de la vie nationale - depuis la science et la technologie jusqu'aux échanges commerciaux.

Research Materials. Contrairement aux SRM's, ils ne sont pas certifiés. Au lieu d'un Certificat, ces RM's sont délivrés avec un "Rapport d'Essais", sous la seule autorité de l'expérimentateur du NBS auteur du 
rapport. Un RM est essentiellement destiné à permettre la poursuite de recherches scientifiques ou techniques sur ce matériau. Pour un RM, le souci majeur est de fournir un matériau homogène, afin que les chercheurs de différents laboratoires soient assurés de travailler sur le même matériau.

Special Reference Materials. Ils différent à la fois des SRM's et des RM's par le fait que NBS ne participe pas à leur caractérisation. Les GM's sont des matériaux de référence produits et certifiés ou garantis par d'autres agences gouvernementales, des organismes de normalisation ou d'autres organisations à but non lucratif. S'il apparait qu'ils correspondent à un intérêt général et s'il n'existe pas d'autres circuits nationaux de distribution, le NBS fait office de distributeur pour ces matériaux. Ce service est ouvert à toute organisation qui qualifie, et qui dispose de matériaux de référence pouvant permettre de résoudre un problème de mesure au niveau national.

\section{Catalogue des SRM's}

La publication d'un nouveau catalogue des Standard Reference Materials du NBS intervient environ tous les trois ans. Il répertorie les nouveaux matériaux disponibles, les matériaux en préparation, et supprime les matériaux épuisés. Entre les publications, la mise à jour du catalogue est assurée par des suppléments annuels, qui répertorient: les prix en vigueur, les nouveaux matériaux, les matériaux épuisés (et proposent des matériaux de remplacement), et les révisions apportées aux Certificats.

Les matériaux répertoriés dans ce catalogue sont séparés en plusieurs grandes classifications: Références de composition chimique (Chemical Composition Standards), Références de propriété physiques (Physical Property Standards), Références à caractère technologique (Engineering Type Standards), "Research Materials", et "Special Reference Materials". Les trois premières classifications correspondent aux seuls SRM's, et sont subdivisées en diverses catégories indiquées à la table de matières.

Les valeurs numériques données dans le catalogue pour décrire les propriétés des matériaux ne sont que de valeurs nominales, à utiliser à titre indicatif pour choisir les matériaux. Elles ne doivent pas être utilisées en lieu et place du Certificate délivré avec le matériau.

Nota: Certains SRM's sont délivrés sans Certificat. Ces exceptions sont précisées à la fois dans la description du SRM et à l'index numérique correspondant.
L'index numérique donne les numéros de SRM, $\mathrm{RM}$ et GM de tous les matériaux répertoriés dans le catalogue, ainsi que, soit la date du dernier Certificat, soit une Nota expliquant l'absence de certificat. Il existe également un index alphabétique. Cet index répertorie principalement les catégories de SRM et l'élément ou la matrice de base, plutôt que sa dénomination précise. Les RM's et les GM's sont regroupés uniquement en grandes rubriques.

\section{Préparation et Disponibilité des Standard Reference Materials}

La préparation de nouveaux SRM's et le renouvellement d'anciens sont effectués continuellement. $\mathrm{Au}$ terme de ces opérations, les utilisateurs potentiels sont avertis de leur disponibilité, et le SRM est décrit dans le supplément au catalogue suivant.

L'objectif, pour les SRM's de renouvellement, est d'assurer une relève avant que le stock du SRM existant ne soit épuisé. Fréquemment, cette condition ne peut être remplie, et, pour un temps, le SRM se trouve en rupture de stock. Quand cela se produit, les demandeurs en sont informés. Eventuellement, ils sont aussi informés de l'existance d'un matériau de remplacement. (voir ci-après "Commandes"). Dès que le lot de renouvellement est disponible, les clients ayant demandé soit le lot antérieur, soit le nouveau, sont rapidement avertis.

Les SRM's de renouvellement ne sont ordinairement pas identiques aux précedénts, mais présentent une grande similitude, en particulier quant aux caractéristiques certifiées. En règle générale, le lot de renouvellement peut être utilizé à la place du précédent. Par exemple, quant le premier acier Bessemer a $0,1 \%$ de carbone a été préparé en 1909 , il a été désigné sous le nom de "Standard Sample No. 8". Les années suivantes, on a préparé un certain nombre de lots de renouvellement, 8a, 8b, etc. . L'actuel SRM 8j, Bessemer Steel (simulé) à $0,1 \%$ de carbone, représente le dixième lot de renouvellement de ce matériau. Alors que ces lots diffèrent quelque peu dans l'analyse détaillée des teneurs, par contre, ils contiennent tous des teneurs relativement élevées en phosphore, soufre et azote, et un faible taux de métaux alliés caractéristique de ce type de matériau.

Une fois le stock épuisé, il devient impossible de fournir les numéros antérieurs à une série de renouvellement. Si la demande pour un matériau est moindre, ou bien si une source équivalente divient disponible, sa production peút être définitivement interrompue. 


\section{Commandes}

Les commandes doivent être adressées a:

Office of Standard Reference Materials

Room B311, Chemistry Building

National Bureau of Standards

Washington, D.C. 20234

Telephone: (301) 921-2045

Les commandes doivent mentionner la quantité (nombre d'unités), la référence figurant au catalogue et le nom du matériau demandé. Par exemple:

1 each, No. 11 h, Basic-Open-Hearth Steel, $0,2 \% \mathrm{C}$.

Les matériaux décrits dans le catalogue sont distribués seulement dans la quantité unitaire précisée ou des multiples de celle-ci.

L'acceptation d'une commande n'implique pas l'acceptation de quelque condition qui serait contraire aux principes, pratiques ou réglementations du National Bureau of Standards ou du Gouvernement des Etats-Unis.

Les commandes reçues pour des matériaux "en rupture de stock" sont annulées quant elles ne portent que sur des articles épuisés. Pour les autres commandes l'expédition des matériaux disponibles est effectuée, et les commandes d'articles épuisés sont annulées. Les rappels de commande ne sont pas acceptés pour les matériaux de rupture de stock. Si un lot de renouvellement du matériau est disponible, il sera automatiquement fourni.

Les prix mentionnés sont en dollars U.S. et sont publiés dans le tarif joint à ce catalogue. Quand de nouveaux tarifs sont appliqués, ils sont envoyés aux utilisateurs qui ont effectué des achats dans les douze mois précédents, et aux personnes ou organisations qui en font la demande. Ces prix sont sujets à révision sans avis préalable, et les commandes sont facturées aux prix en vigueur à la date d'expédition. Il n'est consenti aucune remise sur l'achat de SRM's, RM's, ou GM's.

Il n'est pas nécessaire de joindre a une commande ferme le montant du réglement correspondant à l'achat. Les factures doivent être réglées dans un délai de 30 jours à compter du reçu de la facture. Le paiement des commandes en provenance de l'étranger doit être effectué en dollars U.S., selon l'une des modalités suivantes:

a) Coupons UNESCO

b) Tirage d'un effet sur une banque des Etats-Unis.

c) Transfert bancaire à une banque des EtatsUnis. d) Lettre de crédit sur une banque des Etats-Unis (voir ci-aprés).

e) Mandat international.

La fourniture de factures pro-forma demande un delai de 6 à 8 semaines; ce service n'est assuré que sur demande.

Nota: Les lettres de crédit peuvent être utilisées pour payer à l'avance des SRM's. Elles seront acceptées seulement en provenance d'une banque des EtatsUnis. Ci-dessous sont énumérés les seuls documents qui pourront être fournis:

1) Six factures commerciales.

2) Liste des colis

3) Certificat d'origine

4) Taxe de transport aériene (document qui ne peut être fourni que si l'acheteur paye les frais de transport; s'il est expedié affranchi en paquet poste aérien international, ce type de reçu ne pourra être fourni).

\section{Expéditions: Régime intérieur}

L'expédition de matériaux (sauf pour certains types de matériaux réglementés) à destination des Etats-Unis et du Canada est effectuée au taux d'affranchissement normal (à condition que le paquet ne dépasse pas le poids limite autorisé par les lois et réglements postaux). Cependant, si l'acheteur demande un autre mode d'expédition, elle sera effectuée au compte de l'acheteur. Pour les produits expédiés ainsi, le NBS ne peut payer les taxes de port et les ajouter ensuite à la facture. Les types de matériaux réglementés, tels que hydrocarbures, composés organo-soufrés, gaz comprimés, caoutchoucs, matériaux à base caoutchouc, sources radioactives étalons, et autres matériaux similaires, sont expédiés franco à bord (FOB) de Gaithersburg, Md.

\section{Expéditions: Régime international}

Les réglementations de nombreux pays pour l'importation de SRM's, RM's et GM's comportent de grandes différences; toute tentative d'énumérer toutes les possibilités s'avèrerait impossible. Aussi, quand les modes d'expédition dont nous donnons un aperçu cidessous ne sont pas applicables, les acheteurs sont informés du mode d'expédition adéquat vers leur pays.

La plupart des commandes sont expédiées affranchies en paquet poste aérien international, exceptions faites des types de matériaux réglementés et des envois excédant le poids limite postal autorizé. Les envois se 
rapportant à ces exceptions seront expédiés FOB Gaithersburg, Md, àmoins qu'il ne soit nécessaire d'avoir recours à un agent établi aux Etats-Unis (firme de courtage, d'import-export). Dans ce dernier cas, l'acheteur en est averti et devra désigner un agent de son choix. Le matériau sera alors conditionné pour être expédié outre-mer et sera envoyé à l'agent FOB Gaithersburg, Md.

Nota: Les commandes ou demandes de renseignements écrites en anglais seront plus vite traitées que celles nécessitant une traduction.

\title{
National Bureau of Standards
}

\section{Katalog für Bezugs-Standards verschiedener Materialien, Ausgabe 1981-83}

\begin{abstract}
In diesem Katalog sind alle vom National Bureau of Standards sofort oder in nächster Zeit lieferbaren Bezugs-Standard Materialien (Standard Reference Materials, SRM's), Versuchs-Materialien (Research Materials, RM's) und Spezial-Materialien (Special Reference Materials, GM's) aufgeführt. Sie werden im einzelnen hinsichtlich ihrer garantierten Eigenschaften, der Menge pro Einheit und des Typs beschrieben. Ausserdem werden Hinweise für die Aufgabe von Bestellungen gegeben. Die Preise sind in getrennt zu diesem Katalog erscheinenden Ergänzungslisten enthalten, die jährlich neu herausgegeben werden.
\end{abstract}

Stichworte: Analysis (Analyse)-Certification (Attest)-Characterization (Kennzeihnung)Composition (Zusammensetzung) - Research Materials (Versuch-Materials) - Special Reference Materials (Spezial-Materials) - Standards Reference Materials (Standards Bezugs Materials)

\section{Einleitung}

Das Bezugs-Standaids-Programm des National Bureau of Standards umfasst über 1000 verschiedene Materialien. In der Hauptsache sind es StandardMaterialien (SRM's) deren chemische Zusammensetzung, sowie chemische oder physikalische Eigenschaften bescheinigt werden. Aber auch Versuch-Materialien (RM's) und SpezialMaterialien (GM's) gehören dazu. Alle SRM's, RM's und GM's tragen kennzeichenende Namen und Nummern, mit denen sie jederzeit identifizierbar sind. Somit ist jedes mit einer bestimmten Bezeichnung versehenes SRM, RM oder GM in den geforderten oder vorgesehenen Grenzen mit jeder anderen Probe gleicher Bezeichnung identisch, ausgenommen individuell garantiere Materialien, die zudem durch eine Seriennummer unterschieden werden.

Die ersten vom National Bureau of Standard gelieferten Materialien hiessen Standard-Proben und bestanden aus einer Gruppe von Erzen, Eisenwerkstoffen und Stählen, deren chemische Zusammensetzung bescheinigt wurde. Seit Mitte der 60er Jahre werden diese Materialien als Bezugs-StandardProben geliefert und umfassen ein breites Spektrum chemischer und physikalischer Eigenschaften sowie einen ebenso weiten Bereich von Messwerten.

\section{Definitionen}

Die Bezeichnungen "SRM", "RM" oder "GM" kennzeichnen die Unterschiede bezüglich der mit den einzelnen Proben verbundenen Art der Information und Anwendungsmöglichkeiten.

Standard-Materialien (SRM) werden vom National Bureau of Standards durch bestimmte chemische oder physikalische Eigenschaften gekennzeichnet und mit einem Zertifikat geliefert, in dem die kennzeichnenden Daten aufgeführt sind. Diese Daten werden über einen von drei für die Kennzeichnung festgelegten Wegen ermittelt, und zwar durch Messung der Eigenschaften mittels:

1. eines zuvor bestätigten Referenz-Verfahrens;

2. zweier oder mehrerer voneinander unabhängiger Messmethoden;

3. einer ad hoc Gruppe kooperierender Laboratorien, die technisch dazu in der Lage sind und gründliche Kenntnisse über das zu unterzuchende Material besitzen.

Diese Wege werden im einzelnen in "The Role of Standard Reference Materials in Measurement Sys- 
tems" NBS Special Publication 148 (1975) beschrieben. Standard-Materialien (SRM's) werden als genau gekennzeichnete und garantierte Materialien definiert, die zur Verbesserung der Messtechnik in entsprechenden Mengen hergestellt werden. Drei Hauptzwecke sind es, für die sie hergestellt und eingesetzt werden:

-Die Entwicklung genauer Analysenmethoden

(Referenz-Methoden) zu unterstützen.

-Zur Eichung von Mess-systemen, die dazu dienen

a) den Warenaustausch zu erleichtern,

b) die Grundlage für eine Qualitätskontrolle zu bilden,

c) Leistungsfähigkeit, Wirkingsweise oder Gebrauchswert festzulegen, oder

d) bestimmte Eigenschaften an der Grenze des Entwicklungsstandes zu messen.

-Zur langfristigen Sicherung der Angemessenheit und Integrität von Qualitäts-Prüfverfahren.

Damit tragen die SRM's dazu bei, die Vergleichbarkeit und Genauigheit von Messungen unter den verschiedensten Aspekten des täglichen Lebens-von der Wissenschaft und Technologie bis zu Handel und Wirtschaft-sicherzustellen.

Versuch-Materialien (RM). Anders als bei den SRM's werden hierfür keine Zertifikate erstellt. An deren Stelle tritt ein "Report of Investigation" (Untersuchungsbericht), der unter der ausschliesslichen Verantwortung des ihn erstellenden Mitgliedes des National Bureau of Standards steht. Ein RM soll in erster Linie der Unterstützung der wissenschaftlichen oder technischen Forschung für dieses Material dienen. Hauptgrund für die Ausgabe eines RM ist die Lieferung eines homogenen Materials, das den Forschern in den verschiedenen Laboratorien die Gewähr dafür geben soll, dass sie auch dasselbe Material untersuchen.

Spezial-Materialien (GM). Diese unterscheiden sich von den SRM's und RM's dadurch dass das National Bureau of Standards bei der Festlegung der Kennzeichen für dieses Material nicht mitwirkt. GM's sind Bezungs-Materialien, die von anderen Regierungsstellen, Normen-Ausschüssen oder anderen gemeinnützigen Organisationen hergestellt, beglaubigt oder garantiert werden. Wenn ein öffentliches Interesse an diesen Materialien besteht oder wenn keine entsprechenden Wege der Verteilung im Lande gegeben sind, übernimmt das National Bureau of Standards deren Vertrieb. Dieser Service ist allen Organisation zu- gänglich, die entsprechend qualifiziert sind und im Besitz von Referenz-Materialien sind, die dazu beitragen könnten, ein nationales Messproblem zu lösen.

\section{SRM-Katalog}

Das National Bureau of Standards veröffentlicht etwa alle drei Jahre neue Kataloge mit den von ihm erhältlichen Standard-Bezugs-Materialien, in denen neue zur Verfügung stehende Materialien sowie solche, die sich in Vorbereitung befinden, aufgeführt sind und auch Angaben über nicht weiter lieferbare Proben enthalten sind. Um aber auch in der Zwischenzeit den Katalog auf dem laufenden zu halten, werden alljährlich Ergänzungen herausgegeben. Sie enthalten die jeweils gültigen Preise, neue Materialien, ausgelaufene Materialien (ggf. mit Ersatzvorschlägen) und revidierte Zertifikate.

Die in diesem Katalog aufgeführten Materialien sind in verschieden Hauptgruppen unterteilt: Chemical Composition Standards (Standards für die chemische Zusammensetzung), Physical Property Standards (Standards für physikalische Eigenschaften), Engineering Type Standards (Technische Standards), Research Materials (Versuchs-Materialien) und Special Reference Materials (Spezial-Materialien). Die ersten drei Gruppen umfassen ausschliesslich SRM's und sind in die im Inhaltsverzeichnis aufgeführten Kategorien unterteilt.

Die zur Beschreibung der Material-Eigenschaften im Katalog angegebenen Zahlenwerte sind lediglich Richtwerte und dienen nur als Hinweis bei der Auswahl der Materialien. Sie dürfen nicht anstelle des für das Material ausgegebenen Zertifikats verwendet werden.

Anmerkung: Einige SRM's werden ohne Zertifikat geliefert. Diese Ausnahmen sind sowohl in der SRMBeschreibung als auch im Zahlen-Index entsprechend gekennzeichnet.

Der Zahlenindex enthält die SRM-, RM- und GMNummern aller im Katalog aufgeführten Materialien sowie das Datum des zur Zeit gültigen Zertifikats beziehungsweise eine Begründung, weshalb ein solches nicht vorhanden ist.

Daneben gibt es einen alphabetischen Index. Zum grössten Teil enthält dieser Index die SRM-Gruppen und den Hauptbestandteil oder das Hauptelement des SRM oder zumindest den Namen spezifischer SRM's. RM's und GM's sind lediglich als Gruppen aufgeführt. 


\section{Auftragserteilung}

Aufträge sind $\mathrm{zu}$ richten an

Office of Standard Reference Materials

Room B311, Chemistry Building

National Bureau of Standards

Washington, D.C. 20234

Telephon (301) 921-2045

Die Aufträge müssen enthalten: die gewünschte Menge (Anzahl der Proben), Katalog-Nummer und Bezeichnung des gewünschten Materials. Beispiel: Je eine Nr. 1lh, basischer Siemens-Martin-Stahl, 0,2\% C. Die im Katalog beschriebenen Materialien werden in den angegebenen Mengen oder entsprechenden Vielfachen davon geliefert.

Die Annahme eines Auftrages ist nicht gleichbedeutend mit der Anerkennung irgendeiner zusätzlich in dem Auftrag enthaltenen Vorschrift, die nicht der Politik, der Praxis oder den Regeln des National Bureau of Standards oder der U.S. Regierung entspricht.

Für nicht mehr am Lager befindliche Materialien eingehende Aufträge werden, wenn darin nur solche Materialien enthalten sind, anulliert. Sind in den Aufträgen auch noch andere Materialien enthalten, werden diese geliefert und nur die nicht am Lager befindlichen gestrichen. Für nicht am Lager befindliche Materialien werden auch keine Aufträge zurückgestellt. Sobald die Neuauflage eines Materials zur Verfügung steht, wird automatisch dieses geliefert.

\section{Herstellung und Lieferbarkeit von Standard- Bezugs-Materialien}

Neue oder erneuerte SRM's werden ständig hergestellt. Sobald sie bereit stehen, werden interessierte Anwender direkt über die Lieferbarkeit unterrichtet, und im folgenden Katalog-Nachtrag erfolgt die Beschreibung des SRM's.

Die Uberlegung bezüglich der Neuauflage von SRM's geht davon aus, deren Herstellung bereits in Angriff zu nehmen, bevor die Liefermöglichkeit des bestehenden SRM's erschöpft ist. Manchmal ist dies nicht möglich, und so kann ein SRM vorübergehend vergriffen sein. Wenn dies der Fall ist, werden Besteller dieses Materials darüber informiert und, soweit möglich, auf entsprechenden Ersatz hingewiesen. (Siehe auch unter "Auftragserteilung".) Sobald die Neuauflage zur Verfügung steht, werden alle Kunden, die einen Auftrag auf das frühere oder das neuaufgelegte Material erteilt hatten, sofort benachrichtigt.
Neuaufgelegte SRM's sind gewöhnlich mit ihren Vorgängern nicht identisch, doch sind sie hinsichtlich der spezifizierten Eigenschaften genau so hervorragend. Grundsätzlich kann die Neuauflage anstelle ihres Vorgängers Verwendung finden. Beispiel: Als der erste 0,1 \%ige Bessemer-Kohlestahl im Jahre 1909 hergestellt wurde, erhielt er die Bezeichnung "Standard-Probe Nr. 8". Im Verlauf der folgenden Jahre wurden mehrere Neuauflagen unter der Bezeichnung 8a, 8b usw., hergestellt. Die zur Zeit geltende Nummer für diesen Bessemer-Stahl ist SRM $8 \mathrm{j}$ und stellt die zehnte Neuauflage dieses Materials dar. Obwohl jede dieser neuaufgelegten Serien in der genauen Analyses von den anderen etwas abweicht, weisen jedoch alle den relativ hohen Anteil an Phosphor, Schwefel und Stickstoff und einen geringen Gehalt an Legierungsmetall auf, was für diese Art von Material charakteristisch ist.

Wenn das Lager geräumt ist, ist es nicht mehr möglich, vorangegangene Nummern eines neuaufgelegten Materials zu liefern. Besteht nur ein geringer Bedarf oder ist für ein bestimmtes Material eine andere Lieferquelle vorhanden, kann die Produktion durch das National Bureau of Standards für immer eingestellt werden.

Die Priese sind in U.S.-Dollar festgelegt und in der diesem Katalog beigefügten SRM-Preisliste enthalten. Neue SRM-Preislisten werden nach Erscheinen allen Kunden, die in den vorangegangenen 12 Monaten käufe getätigt haben, sowie Personen oder Organisationen, die danach verlangt haben, zugestellt. Die Preise sind unverbindlich und können ohne diesbezügliche Nachricht Änderungen unterliegen. Bei Aufträgen werden stets die im Augenblick der Lieferung gültigen Preise in Rechnung gestellt. Auf SRM's, RM's und GM's werden keinerlei Abzüge gewährt.

Vorauszahlung bei Auftragserteilung ist nicht notwendig. Für die Begleichung einer Rechnung werden 30 Tage Ziel nach Erhalt gewährt. Die Begleichung ausländischer Aufträge muss in U.S.-Dollar erfolgen. Dies kann in folgender Weise geschehen:

a) mit UNESCO-Kupons,

b) durch Scheck einer U. S. Bank,

c) durch Bank-zu-Bank-Uberweisung auf eine U. S. Bank,

d) durch Kreditbrief auf eine U. S. Bank (siehe unten) oder

e) durch internationale Postanweisung.

Die Ausstellung von proforma-Rechnungen erfordert gewöhnlich 6 bis 8 Wochen und wird deshalb nur auf Wunsch vorgenommen. 
Anmerkung: Für die Vorauszahlung können Kreditbriefe verwendet werden. Sie werden nur von Banken in den Vereinigten Staaten angenommen.

Folgende Begleitdokumente werden den Sendungen beigefügt:

1. Sechs Waren-Rechnungen

2. Packzettel

3. Ursprungs-Zertifikat

4. Luftfracht-Rechnung (Sie wird nur ausgestellt, wenn das Material gegen Nachnahme versandt wird. Erfolgt der Versand portofrei mit der internationalen Luftpaketpost, können keine Empfangsbestätigungen gegeben werden.)

\section{Inland-Versand}

Nach den USA und Canada erfolgt der Versand (einige beschränkte Gruppen ausgenommen) normalerweise portofrei, vorausgesetzt dass das Gewicht des Pakets nicht die postalisch vorgeschriebenen Gewichtsgrenzen überschreitet. Verlangt der Käufer jedoch eine andere Versandart, so erfolgt die Lieferung gegen Nachnahme. Bei Nachnahmesendungen kann das National Bureau of Standards nicht die Portokosten usw. auf der Rechnung vermerken. Bestimmte Gruppen wie Kohlenwaseerstoffe, organische Schwefelverbindungen, komprimierte Gase, Gummi,
Gummi-Zusatz-Materialien, radioaktive Standards und ähnliche Materialien werden fob Gaithersburg, Md. (USA) zum Versand gebracht.

\section{Versand ins Ausland}

Die Einfuhr-Vorschriften für SRM's, RM's und GM's sind für die einzelnen Länder sehr unterschiedlich. Es wäre zwecklos, alle möglichen Abweichungen hier zusammenzustellen. Deshalb werden die Käufer überall dort, wo die nachfolgenden Versandpraktiken nicht anwendbar sind, über die für ihr Land beste Versandform informiert.

Die meisten Aufträge werden portofrei mit der internationalen Luftpaketpost versandt. Ausgenommen sind Proben der beschränkten Gruppen und Sendungen, die das für Fusipakkete zulässige Gewicht überschreiten. In diesen Fällen erfolgt der Versand fob Gaithersburg, Md. (USA), es sei denn, ein Vertreter (Transport-oder Makler-Firma) mit sitz in den USA gewünscht wird. Wenn dies der Fall ist, wird der Käufer entsprechend unterrichtet und aufgefordert, einen Vertreter seiner Wahl zu benennen. In diesem Falle wird das Material für den ÜberseeVersand verpackt und dem Vertreter fob Gaithersburg, Md. (USA) zugestellt.

Anmerkung: Aufträge und Anfragen in englischer Sprache werden schneller abgewickelt als solche, die erst einer Übersetzung bedürfen. 


\section{Tipuartment of Commerre and Tiabar \\ BUREAU OF STANDARDS \\ THashingtan}

Bureau Circular No. 11

February 1, 1906.

Under the provisions of the act of Congress establishing the Bureau of Standards, the Bureau is authorized, among other things, to determine "physical constants and the properties of materials, when such data are of great importance to scientific or manufacturing interests."

\section{DESCRIPTION OF SAMPLES.}

For several years the American Foundrymen's Association has prepared and distributed samples of standardized irons. By an agreement with that association the samples which have been prepared under its direction have been transferred to the Bureall of Standards, and this Bureau will, in the future, have charge of the preparation and distribution of such samples, and will also, as soon as practicable, undertake the preparation and distribution of samples of standardized steels. The samples now ready for distribution are as follows:

Sample $A$ (iron).-The analysis of this sample gives total carbon, graphite, and combined carbon. The sample has been ground to pass a 40-mesh sieve to secure greater homogeneity.

Sample B (iron). - The analysis of Sample B gives total carbon, graphite, and combined carbon, phosphorus (by three methods), silicon, titanium, total sulphur and sulphur by evolution as hydrogen sulphide, manganese, and copper. The silicon and manganese in this iron are low, and the phosphorus is within the Bessemer limit.

Sample $C$ (iron).-The analysis of Sample $\mathrm{C}$ gives the same determinations as in Sample $\mathrm{B}$, with the exception of copper. The silicon, phosphorus, and manganese in the iron are mediuin.

Sample $D$ (iron).-The analysis of Sample $\mathrm{D}$ gives the same determinations as in Sample C. The silicon, phosphorus, and manganese in the iron are high.

Sample 0.5 (stecl).-At present only a single sample of steel has been prepared. This is designated Sample 0.5. The analysis of this sample gives total carbon, silicon, total sulphur and sulphur by evolution as hydrogen sulphide, phosphorus (by two methods), and manganese. The sample is an open-hearth steel of about 0.5 per cent carbon, is almost free from silicon, and is low in sulphur. As the sample is not as homogeneous as is desirable, determinations of its carbon and manganese in different parts of the ingot, as well as the average, are given. A better sample will be obtained as soon as possible.

\section{METHODS OF ANALYSIS.}

Five independent analyses, made by different individuals, are given for Samples B, C, and $\mathrm{D}$, and three independent analyses for the sample of steel. The methods of analysis used at the Bureau of Standards were, in brief outline, as follows:

Total carbon.-Two grams of the iron were dissolved in $100 \mathrm{cc}$ of a solution of copper potassium chloride containing 300 grams of the salt and $100 \mathrm{cc}$ of concentrated hydrochloric acid per liter. The solution was stirred by a mechanical stirrer. When solution was complete, 5 per cent more of concentrated hydrochloric acid was added and the carbon filtered off on a platinum boat, washed ${ }^{-}$with water and dilute hydrochloric acid, dried 
at $100^{\circ}$ and burned in a current of oxygen in a porcelain tube, using a ten-burner combustion furnace.

Graphite.-Two grams of iron were dissolved in dilute nitric acid (sp. gr. 1.20), using $35 \mathrm{cc}$ and heating very gently. The residue was filtered off on a platinum boat, washed out with hot water, then with a hot solution of potassium hydroxide (sp. gr. 1.10), followed by dilute hydrochloric acid and finally by hot water. After drying at $100^{\circ}$, the graphite was burned in the same manner as the total carbon.

Combined carbon.-This was obtained by subtracting graphitic carbon from total carbon.

Silicon.-Two grams of iron were dissolved in $30 \mathrm{cc}$ of nitric acid (sp. gr. 1.20) and the solution evaporated to dryness and baked on a hot plate until the ferric nitrate was decomposed. The dish was then cooled and $30 \mathrm{cc}$ of strong hydrochloric acid added to dissolve the ferric oxide. When solution was complete, the acid was evaporated and the residue again baked on the hot plate. Thirty cubic centimeters of strong hydrochloric acid were added and the dish heated until all iron had gone into solution, when hot water was added and the residue filtered off and washed with hot water and dilute hydrochloric acid. The filter and contents were then placed in a platinum crucible and the filter paper' and graphite burned off and the residue strongly ignited over the blast. After weighing, the silica was volatilized with hydrofluoric acid with the addition of a little sulphmric acid, and the residue ignited. The difference between the two weights was called "silica," and the silicon calculated therefrom.

Titanium.-Titaniun was determined by treating 5 grams of iron with 40 ce of hydrochloric acid $(1: 1)$ and heating until all iron had gone into solution. Dissolving in this manner it was found that all but a negligible quantity of titanium remained in the insoluble residue. The filtrate was tested for titanium by extracting the iron with ether after oxidation with a small amount of nitric acid, using the method of Rothe (Stahl und Eisen, 12, 1052 (189?), and 13, 333 (1893), ) and adding hydrogen peroxide to the extracted solution, after expelling the ether and oxidizing with nitric acid. In all cases only a faint coloration was obtained. The insoluble residue was filtered off and washed with hot water, and the filter paper and carbonacens matter were burned. The residue in the crucible was treated with hydrofluoric acid and a little sulphuric acid, and all silicon volatilized. The residue was fusod with sodium carbonate, treated with water and acidified with sulphuric acid. A sufficient amount of ferric alum was added to the standard titanium solution to give the same tint as the sample when they were at the same dilution, for it was found that the residue from the silica always contained a little iron along with the titanium. Hydrogen peroxide was added to the solution and standard and the comparison made in a Wolff colorimeter.

Phosphorus.-Two grams of iron were dissolved in nitric acid (sp. gr. 1.133) and boiled until yellow fumes no longer came off. Ten cubic centimeters of a permanganate solution (15 grams to 1 liter) were added, and the boiling continued. Sodium sulphite solution was added to dissolve the oxide of manganese, and the solution again boiled and filtered. After cooling, $10 \mathrm{cc}$ of ammonia (sp. gr. 0.96) were added, the solution agitated, and when the temperature was at $40^{\circ}$, 40 ec of molybdate solution (prepared according to Blair's "Chemical Analysis of Iron," 5th ed., p. 97) were added and the solution sliaken vigorously for five minutes. After settling out, the yellow precipitate was filtered off and washed with acid aminonium sulphate (prepared according to Blair) until the washings did not react for iron or molyblic acid. The precipitate was treated on the filter with $25 \mathrm{cc}$ of ammonia ( $5 \mathrm{cc}$ of ammonia sp. gr. 0.90 to $20 \mathrm{cc}$ of water). The filter was washed out with water and 10 ec of strong sulphuric acid added to the filtrate, which was then run through the reauctor and titrated against a permanganate solution containing about 2 grams to the liter. A blank was always run with the reductor before each 
titration, using the same quantities of ammonia and sulphuric acid. The insoluble residue was always tested for phosphorus.

Sulphur, gravimetric.--Five grains of iron were dissolved in a 400 cc Erlenmeyer flask, using 50 ec of strong nitric acid. A little sodium carbonate was then added, the solution evaporated to dryness, and the residue baked for an hour on the hot plate. The residue was dissolved in $30 \mathrm{cc}$ of strong hydrochloric acid and the solution again evaporated to dryness and baked. Thirty cubic centimeters of strong hydrochloric acid were then added, and, after the iron had dissolved, the solution was evaporated to sirupy consistency and from 2 to 4 cc of strong hydrochloric acid added. When all iron was in solution, between 30 and $40 \mathrm{cc}$ of hot water were added, and the solution was filtered and the residue washed with hot water. The sulphur was precipitated in the cold solution with $10 \mathrm{cc}$ of a 10 per cent solution of barium chloride. The precipitate was filtered off after standing for twenty-four hours. The insoluble residue was ignited, fused with sodium carbonate and nitrate, extracted with water, acidified with hydrochloric acid, evaporated to dryness, extracted with water and a few drops of hydrochloric acid, and the solution precipitated with barium chloride, the sulphur obtained in this way being added to that from the main filtrate. All evaporations were carried on over electrically heated hot plates to avoid sulphur from gas flames, and careful blank determinations were made with all reagents.

Sulphur evolved as hydrogen sulphide.-Five grams of iron were dissolved in $60 \mathrm{cc}$ of hydrochloric acid $(1: 1)$ in the usual evolution apparatus, the air of which had been previously displaced by hydrogen. The hydrogen sulphide evolved was absorbed in a solution of sodium hydroxide (sp. gr. 1.1). The solutions in the absorbing vessels were poured into a beaker and diluted to about $500 \mathrm{cc}$, acidified with hydrochloric acid, a little potassium iodide added, and titrated with a standard iodine solution, using freshly prepared starch solution as an indicator. Blanks were always made. The iodine solution was standardized against anhydrous sodium thiosulphate (prepared according to Young, J. Am. Chem. Soc., 26, 1028 (1904).)

Manganese.-Two grams of iron were dissolved in $30 \mathrm{cc}$ of nitric acid (sp. gr. 1.20), and after filtration the solution was evaporated to about $15 \mathrm{cc}$. Two grams of potassium chlorate were added and the boiling continued for fifteen minutes; $10 \mathrm{cc}$ more of strong nitric acid were then added, with 1 gram of potassium chlorate, and the solution boiled ten minutes longer. The precipitated manganese was filtered on asbestos, washed with strong nitric acid till free from iron, and then washed with water till free from acid. The asbestos pad holding the manganese was transferred to a flask and shaken up with $25 \mathrm{cc}$ of a solution of ammonium ferrous sulphate containing 5 per cent sulphuric acid, and when solution of the manganese was completed the excess of ferrous iron was determined by titration with a permanganate solution.

Manganese was also determined as follows: Two grams of iron were dissolved in $30 \mathrm{cc}$ of nitric acid (sp. gr. 1.20). When solution was complete, the solution was nearly neutralized with sodium carbonate, and zinc oxide emulsion added. After standing a few minutes, an excess of zinc oxide was added, and the solution made up to a definite volume and one-half or three-fifths filtered off through a dry filter and diluted to about $200 \mathrm{cc}$. To this were added $20 \mathrm{cc}$ of sodium acetate solution (30 grams crystalized sodium acetate, $30 \mathrm{cc}$ of 30 per cent acetic acid, $170 \mathrm{cc}$ of water) and $40 \mathrm{cc}$ of bromine water. The solution was warmed gently until all manganese had separated. The precipitate was filtered, washed, dissolved, and titrated as before.

Copper.-Ten grams of iron were treated with 10 per cent sulphuric acid. All the copper was found in the insoluble residue. This was digested with aqua regia, and excess of ammonia added. The solution was filtered and compared with a standard copper solution treated with the same excess of ammonia. 
The methods used by the other analysts were as follows: Booth, Garrett, and Blair used the acetate method for gravimetric phosphorus; Bamber's fusion method for gravimetric sulphur; also an evolution method, absorbing hydrogen sulphide in an alkaline solution of a lead salt, with fusion of the residue; the nitric acid and potassium chlorate separation, with weighing as manganese pyrophosphate, for manganese, and Drown's method for silicon.

Andrew S. McGreath used for silicon both Drown's method and the method of fusion of the residue from the nitric acid solution for phosphorus with sodium carbonate, evaporating the solution of the fusion with hydrochloric acid. He determined sulphur by solution in nitric acid, conversion of this to a chloride solution and precipitation by barium chloride in a small volume. For phosphorus both the acetate and molybdate methods were used, and for manganese the chlorate method.

Cremer and Bicknell used the molybdate separation for phosphorus, weighing as pyrophosphate; oxidation with nitrohydrochloric and precipitation in the ferric chloride solution for sulphur, and the acetate separation and weighing as $\mathrm{Mn}_{3} \mathrm{O}_{4}$ for manganese.

Albert W. Smith used the molybdate separation for phosphorus, weighing as magnesium pyrophosphate; oxidation with nitrohydrochloric acid and precipitation in the ferric chloride solution for sulphur; the acetate separation and weighing as manganese pyrophosphate for manganese, and Drown's method for silicon.

In all cases the total carbon was determined by solution in copper-potassium chloride solution and combustion of the residue, and the graphite by solution in dilute acid and combustion.

\section{USE OF THE SAMPLES.}

Great pains have been taken to make the contents of all the bottles uniform, and the agreement of the analyses indicates that these efforts have been successful. In shipping, however, the fine and coarse particles in the bottles become segregated so that it is of the greatest importance that the contents of each bottle be thoroughly mixed again before any is used for analysis. Each bottle will be labeled with its letter only, but gummed labels containing the average of the analyses by the different chemists will be furnished with each sample, and also certificates giving the complete analysis of each chemist, as it is believed that many will desire to know how close an agreement has been secured in these analyses.

FEES.

The following schedule of fees has been adopted for samples of the same or different irons or steels :

SCHEDUle 101.-Samples of Standardized Iron and Steel.

(a) Single samples, of 150 grams, each ...

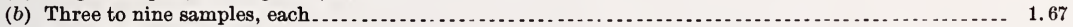

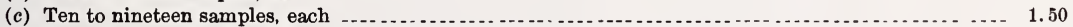

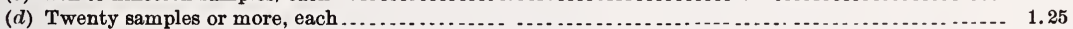

Orders for samples should be accompanied by a remittance, which may be by check, draft, or post-office order, and should be made payable to the Bureau of Standards.

S. W. Stratton, Director.

Approved:

V. H. Metcalf, Secretary. 


\section{CERTIFIED CHEMICAL COMPOSITION STANDARDS}

\section{Steels (Chip Form)}

These SRM's were prepared for the steel industry primarily for use with methods involving sample solutions in checking chemical methods of analysis for both production control and customer acceptance. These SRM's consist of nominal composition steel alloys selected to provide a wide range of analytical values for various elements of vital concern to the chemist. They are furnished in 150-gram units (unless otherwise noted) as chips usually sized between 0.4 to $1.2 \mathrm{~mm}$, prepared from selected portions of commercial ingots.

Plain Carbon Steels

\begin{tabular}{|c|c|c|c|c|c|c|c|}
\hline \multirow{3}{*}{ SRM } & \multirow{3}{*}{ Type } & \multicolumn{6}{|c|}{ Chemical Composition (Nominal Weight Percent) } \\
\hline & & \multirow{2}{*}{$\mathrm{C}$} & \multirow{2}{*}{ Mn } & \multirow{2}{*}{$\mathbf{P}$} & \multicolumn{2}{|c|}{$\mathrm{S}$} & \multirow{2}{*}{$\mathrm{Si}$} \\
\hline & & & & & Grav & Comb & \\
\hline $8 \mathrm{j}$ & Bessemer (simulated), 0.1C & 0.081 & 0.505 & 0.095 & & 0.077 & 0.058 \\
\hline $11 \mathrm{~h}$ & BOH, $0.2 \mathrm{C}$ & .200 & .510 & .010 & & .026 & $.21_{1}$ \\
\hline $12 \mathrm{~h}$ & BOH, $0.4 \mathrm{C}$ & .407 & .842 & .018 & & .027 & .235 \\
\hline $13 \mathrm{~g}$ & $\mathrm{BOH}, 0.6 \mathrm{C}$ & .61 & .85 & .006 & & .031 & $.35_{5}$ \\
\hline $14 \mathrm{e}$ & BOH, $0.8 \mathrm{C}$ & .753 & .404 & .008 & & .039 & .177 \\
\hline $15 \mathrm{~g}$ & BOH, $0.1 \mathrm{C}$ & .094 & .485 & .005 & & .026 & .095 \\
\hline $16 \mathrm{e}$ & ВOH, 1.1C & 1.09 & .381 & .028 & ............... & .029 & $.2 \mathrm{O}_{2}$ \\
\hline $19 \mathrm{~g}$ & $\mathrm{AOH}, 0.2 \mathrm{C}$ & 0.223 & .554 & .046 & 0.032 & .033 & .186 \\
\hline $20 \mathrm{~g}$ & AISI 1045 & .462 & .665 & .012 & 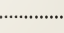 & .028 & .305 \\
\hline $152 \mathrm{a}$ & $\mathrm{BOH}, 0.5 \mathrm{C}$ (Tin bearing) & .486 & .717 & .012 & & .030 & .202 \\
\hline 178 & 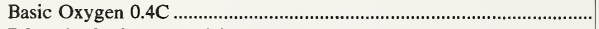 & .395 & .824 & .012 & . & .014 & .163 \\
\hline 335 & $\mathrm{BOH}, 0.1 \mathrm{C}$ (Carbon only) $300 \mathrm{~g}$ & .092 & & & & & \\
\hline 337 & BOH, 1.1C (Carbon only) $300 \mathrm{~g}$ & 1.07 & .............. & & & & 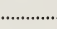 \\
\hline 368 & 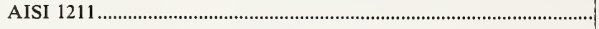 & 0.089 & .82 & .084 & 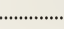 & .132 & .007 \\
\hline
\end{tabular}

\begin{tabular}{|c|c|c|c|c|c|c|c|c|c|c|c|}
\hline SRM & $\mathrm{Cu}$ & $\mathrm{Ni}$ & $\mathrm{Cr}$ & V & Mo & Co & $\mathrm{Ti}$ & Sn & $\underset{\text { (total) }}{\mathrm{Al}}$ & $\mathrm{N}$ & Other \\
\hline $8 \mathrm{j}$ & 0.020 & 0.113 & 0.047 & 0.015 & 0.038 & & & & & & \\
\hline $11 \mathrm{~h}$ & .061 & .028 & .025 & .001 & .......................... & ....................... & 0.004 & & …….......... & ...................... & 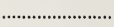 \\
\hline $12 \mathrm{~h}$ & .073 & .032 & .074 & .003 & .006 & ......................... & 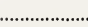 & .................... & $(0.038)$ & 0.006 & ․․․․……… \\
\hline $13 \mathrm{~g}$ & .066 & .061 & .050 & .001 & ................ & & …………....... & ................... & $.04_{\mathrm{s}}$ & ․․…... & 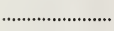 \\
\hline $14 \mathrm{e}$ & .072 & .053 & .071 & .002 & .013 & & & & .060 & & \\
\hline $15 \mathrm{~g}$ & .036 & .017 & .028 & .001 & & & & & & & \\
\hline $16 \mathrm{e}$ & .052 & .072 & .118 & .002 & ........... & & & & ............. & $\ldots$ & $\ldots$ \\
\hline $19 \mathrm{~g}$ & .093 & .066 & .374 & .012 & .013 & 0.012 & .027 & 0.008 & .031 & ...... & $\mathrm{Nb} 0.026$ \\
\hline $20 \mathrm{~g}$ & .034 & .034 & .036 & .002 & .008 & & 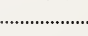 & $\ldots$ & .040 & $\ldots$ & $\ldots$ \\
\hline $152 a$ & .023 & .056 & .046 & .001 & .036 & & & .032 & & & ................. \\
\hline 178 & .032 & .010 & .016 & .001 & .003 & & & & & & …………........... \\
\hline 335 & & & $\ldots$ & .1...... & & ...................... & $\ldots$ & ....................... & .................... & ..................... & ……………..... \\
\hline 337 & & & & & & & & & & & \\
\hline 368 & .010 & .008 & .030 & .001 & .003 & .................... & ................... & ..................... & ............................ & .010 & . \\
\hline
\end{tabular}




\begin{tabular}{|c|c|c|c|c|c|c|c|c|c|c|}
\hline \multirow{3}{*}{ SRM } & \multirow{3}{*}{ Type } & \multicolumn{9}{|c|}{ Chemical Composition (Nominal Weight Percent) } \\
\hline & & \multirow{2}{*}{ (Other Forms) } & \multirow{2}{*}{$\mathrm{C}$} & \multirow{2}{*}{ Mn } & \multirow{2}{*}{$\mathbf{P}$} & \multicolumn{2}{|c|}{ S } & \multirow{2}{*}{$\mathrm{Si}$} & \multirow{2}{*}{$\mathrm{Cu}$} & \multirow{2}{*}{$\mathrm{Ni}$} \\
\hline & & & & & & Gray & Comb & & & \\
\hline $30 \mathrm{f}$ & $\mathrm{Cr}-\mathrm{V}(\mathrm{SAE} 6150) \ldots \ldots$ & & 0.490 & 0.79 & 0.011 & & 0.009 & 0.243 & 0.074 & 0.070 \\
\hline $32 \mathrm{e}$ & $\mathrm{Ni}-\mathrm{Cr}(\mathrm{SAE} 3140) \ldots \ldots \ldots \ldots . . . .$. & ........................... & .409 & .798 & .008 & 0.022 & .021 & .278 & .127 & 1.19 \\
\hline 33d & Ni-Mo (SAE 4820)......... & & .173 & .537 & .006 & .010 & .011 & .253 & .123 & 3.58 \\
\hline $36 \mathrm{~b}$ & $\mathrm{Cr} 2-\mathrm{Mol}$ & & .114 & .404 & .007 & & .019 & .258 & .179 & 0.203 \\
\hline $72 \mathrm{~g}$ & 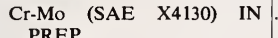 & $\ldots \ldots . . .$. & ……...... & & & 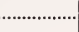 & & & & ….. \\
\hline $100 \mathrm{~b}$ & Manganese (SAE T1340) & & & 180 & & & & & & \\
\hline 105 & High-Sulfur $0.2 \mathrm{C}$ (Carbon & . & .193 & 1.89 & .023 & .029 & $\begin{array}{l}.028 \\
(.60)\end{array}$ & .210 & .064 & .030 \\
\hline & $\begin{array}{l}\text { only). } \\
\text {. }\end{array}$ & & & & & & & & & \\
\hline $106 \mathrm{~b}$ & Cr-Mo-Al (Nitralloy G) ................ & & 326 & 0.506 & .008 & .016 & .017 & .274 & .117 & .217 \\
\hline $125 \mathrm{~b}$ & High-Silicon............................ & 1134 & .028 & .278 & .029 & & .008 & 2.89 & .071 & .038 \\
\hline $129 \mathrm{c}$ & High-Sulfur ................................... & 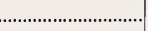 & .125 & .769 & .076 & .................. & .245 & 0.020 & .013 & .251 \\
\hline $131 \mathrm{c}$ & Low Carbon-Silicon $(100 \mathrm{~g}) \ldots$ & & .0029 & & & & .020 & & & \\
\hline $139 \mathrm{~b}$ & 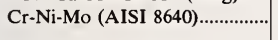 & 1222 & .403 & .778 & .013 & wer & .019 & .242 & .097 & .510 \\
\hline 155 & Cr0.5-W0.5 & & .905 & 1.24 & .015 & .010 & .011 & .322 & .083 & .100 \\
\hline 179 & 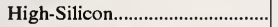 & 1135 & .027 & 0.094 & .006 & & .026 & 3.19 & .056 & .050 \\
\hline 291 & Cr-Mo (ASTM A213) & $\ldots$ & .177 & .55 。 & .008 & & .020 & 0.23 。 & .047 & .065 \\
\hline 293 & $\mathrm{Cr}-\mathrm{Ni}-\mathrm{Mo}$ (AISI 8620)................... & & .222 & $.96_{0}$ & .018 & ............ & .022 & $.30_{0}$ & .032 & .48 o \\
\hline 361 & AISI 4340 & $661,1095,1261$ & .383 & .66 & .014 & ................. & .015 & .222 & .042 & 2.00 \\
\hline 362 & AISI 94B17 (Mod) & $662,1096,1262$ & .160 & 1.04 & .041 & & .038 & .39 & .50 & 0.59 \\
\hline 363 & $\mathrm{Cr}-\mathrm{V}(\mathrm{Mod})$ & $663,1097,1263$ & .62 & 1.50 & $.02_{9}$ & .................... & .009 & .74 & .10 & .30 \\
\hline 364 & High Carbon (Mod) ........................ & $664,1098,1264$ & .87 & $0.25_{5}$ & $.01_{\theta}$ & (n.................. & .02 & $.06_{5}$ & .24 & $.14_{4}$ \\
\hline
\end{tabular}

\begin{tabular}{|c|c|c|c|c|c|c|c|c|c|c|c|c|c|}
\hline SRM & $\mathrm{Cr}$ & V & Mo & W & Co & $\mathrm{Ti}$ & As & Sn & $\underset{\text { (total) }}{\mathrm{A} 1}$ & $\mathrm{Nb}$ & $\mathrm{Ta}$ & $\mathrm{Zr}$ & $\mathbf{N}$ \\
\hline $30 \mathrm{f}$ & 0.95 & 0.18 & & & & & & & & & & & 0.010 \\
\hline $32 \mathrm{e}$ & .678 & .002 & 0.023 & ...………....... & …………....... & ………… & ..................... & $(0.011)$ & …………..... & ........................ & ................ & 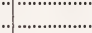 & 0.009 \\
\hline $33 \mathrm{~d}$ & .143 & .002 & .246 & & ……….......... & & & (5) & & & & & $(.011)$ \\
\hline $36 \mathrm{~b}$ & 2.18 & .004 & .996 & 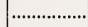 & ..................... & .................. & $\ldots \ldots \ldots$ & ................... & ....................... & ...................... & ..................... & ..................... & ........................ \\
\hline $72 \mathrm{~g}$ & & ............. & ................ & & (2) & & 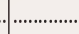 & ..................... & ……........... & & ............... & .................... & 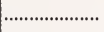 \\
\hline $100 \mathrm{~b}$ & 0.063 & .003 & .237 & & & & & & & & & & \multirow[t]{2}{*}{.004} \\
\hline 105 & & & & & & & & & \multirow{4}{*}{$\begin{array}{l}1.07 \\
0.329\end{array}$} & & & & \\
\hline $106 \mathrm{~b}$ & 1.18 & .003 & .199 & $\ldots .$. & ...................... & .............. & $\ldots \ldots$ & & & $\ldots$ & & ........ & \multirow{2}{*}{. } \\
\hline $125 \mathrm{~b}$ & 0.019 & ................. & .008 & 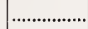 & ...………...... & ..................... & ...…….......... & .003 & & ............. & ........ & ...................... & \\
\hline $129 c$ & .014 & .012 & .002 & & & & & & & & & & \multirow{3}{*}{.007} \\
\hline $139 \mathrm{~b}$ & .488 & .004 & .182 & ........... & .............. & 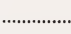 & 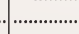 & 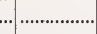 & ..................... & ……. & ........... & ...................... & \\
\hline 155 & .485 & .014 & .039 & 0.517 & & & & & & & & & \\
\hline 179 & .022 & $<.01$ & .014 & .................. & & ........... & ................... & .004 & .0028 & & & & 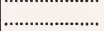 \\
\hline 291 & 1.33 & ..................... & $.53_{\theta}$ & & & & & & .002 & & & & ……....... \\
\hline 293 & $0.51_{0}$ & .004 & .204 & & & & & & .039 & & & & \\
\hline 361 & $.69_{4}$ & .011 & .19 & .017 & 0.032 & 0.020 & 0.017 & .010 & $.02_{1}$ & 0.022 & 0.020 & 0.009 & $(.0037)$ \\
\hline 362 & .30 & .040 & .068 & .20 & .30 & .084 & $.09_{2}$ & .016 & $.09_{5}$ & .29 & .20 & .19 & $(.00404)$ \\
\hline 363 & 1.31 & .31 & .028 & .046 & .048 & .050 & .010 & $.10_{4}$ & .24 & .049 & $(.053)$ & .049 & $(.0041)$ \\
\hline 364 & $0.06_{3}$ & $.10_{5}$ & .49 & .10 & .15 & .24 & $.05_{2}$ & .008 & $(.008)$ & $.15_{7}$ & .11 & .068 & $(.0032)$ \\
\hline SRM & B & $\mathbf{F}$ & b & $\mathrm{Sb}$ & $\mathrm{Bi}$ & & $\mathrm{Ag}$ & $\mathrm{Se}$ & $\mathrm{Te}$ & & $\mathrm{Ce}$ & $\mathrm{La}$ & $\mathrm{Nd}$ \\
\hline 361 & $0.0003_{7}$ & $(45.6$ & & 0.0042 & $(0.0004)$ & & 0004 & $(0.004)$ & $(0.0006)$ & & 040 & $(0.001)$ & $0.0007_{s}$ \\
\hline 362 & .0025 & & $004_{8}$ & .013 & $(.002)$ & & 0011 & $(.0012)$ & $(.0011)$ & & 019 & $(.001)$ & $.0007_{\mathrm{s}}$ \\
\hline 363 & $.0007_{\theta}$ & & $18_{6}$ & .002 & $(.0008)$ & & 0037 & $(.00016)$ & $(.0009)$ & & 030 & $(.002)$ & .0012 \\
\hline 364 & .0106 & & $23_{0}$ & .034 & $(.0009)$ & & 00002) & $(.00021)$ & $(.0002)$ & & $\mathrm{OOS}_{7}$ & $(.0002)$ & $.0001_{8}$ \\
\hline
\end{tabular}




\begin{tabular}{|c|c|c|c|c|c|c|c|c|c|c|}
\hline SRM & $\mathrm{Ca}$ & $\mathrm{Mg}$ & $\mathrm{Zn}$ & Pr & $\mathrm{Ge}$ & 0 & $\mathbf{H}$ & $\mathrm{Au}$ & $\mathrm{Hf}$ & $\mathrm{Sr}$ \\
\hline 361 & $0.0001_{0}$ & $0.0002_{6}$ & $(0.0001)$ & $(0.0003)$ & {$[0.006]$} & $(0.0009)$ & $(<0.0005)$ & $(<0.00005)$ & $(0.0002)$ & $(<0.0005)$ \\
\hline 362 & $.0002_{1}$ & $.0006_{\mathrm{B}}$ & $(.0005)$ & $(.0003)$ & {$[.002]$} & $(.00107)$ & $(<.0005)$ & $(<.00005)$ & $(.0003)$ & ............................ \\
\hline 363 & $.0002_{2}$ & $.0006_{2}$ & $(.0004)$ & $(.0004)$ & {$[.010]$} & $(.00066)$ & $(<.0005)$ & .0005 & $(.0005)$ & $\ldots$ \\
\hline 364 & .00003 & .00016 & {$[.001]$} & $(.0001)$ & {$[.003]$} & $(.0010)$ & $(<.0005)$ & .0001 & $(.0013)$ & 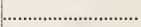 \\
\hline
\end{tabular}

High Alloy Steels

\begin{tabular}{|c|c|c|c|c|c|c|c|c|}
\hline \multirow{3}{*}{ SRM } & \multirow{3}{*}{ Type } & \multicolumn{7}{|c|}{ Chemical Composition (Nominal Weight Percent) } \\
\hline & & \multirow{2}{*}{$\mathrm{C}$} & \multirow{2}{*}{ Mn } & \multirow{2}{*}{$\mathbf{P}$} & \multicolumn{2}{|c|}{ S } & \multirow{2}{*}{$\mathrm{Si}$} & \multirow{2}{*}{$\mathrm{Cu}$} \\
\hline & & & & & Grav & Comb & & \\
\hline $126 \mathrm{c}$ & High-Nickel (36\% Ni) & 0.026 & 0.47 & 0.004 & & 0.006 & 0.19 & 0.040 \\
\hline 344 & Cr15-Ni7-Mo2-All & .69 & .57 & .018 & & .019 & .395 & .106 \\
\hline 345 & 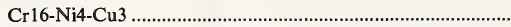 & .048 & .224 & .018 & 0.012 & .012 & .610 & 3.44 \\
\hline 348 & 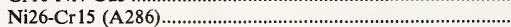 & .044 & 1.48 & .015 & & .002 & .54 & 0.22 \\
\hline
\end{tabular}

\begin{tabular}{|c|c|c|c|c|c|c|c|c|c|c|c|}
\hline SRM & $\mathrm{Ni}$ & $\mathrm{Cr}$ & V & Mo & Co & $\mathrm{Ti}$ & $\begin{array}{c}\mathrm{Al} \\
\text { (Total) }\end{array}$ & $\mathrm{Nb}$ & $\mathrm{Ta}$ & B & $\mathrm{Fe}$ \\
\hline $126 \mathrm{c}$ & 36.05 & $0.06_{4}$ & 0.001 & 0.011 & 0.008 & & & & & & \\
\hline 344 & 7.28 & 14.95 & .040 & 2.40 & & \multirow[t]{2}{*}{0.076} & \multirow[t]{2}{*}{1.16} & & \multirow[t]{2}{*}{0.002} & 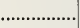 & $\ldots \ldots$ \\
\hline 345 & 4.24 & 16.04 & .041 & 0.122 & .089 & & & \multirow[t]{2}{*}{0.231} & & & \\
\hline 348 & 25.8 & 14.54 & .25 & 1.3 & & 2.24 & 0.23 & & 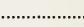 & 0.0031 & 53.3 \\
\hline
\end{tabular}

Stainless Steels

\begin{tabular}{|c|c|c|c|c|c|c|c|c|c|}
\hline \multirow{3}{*}{ SRM } & \multirow{3}{*}{ Type } & \multicolumn{8}{|c|}{ Chemical Composition (Nominal Weight Percent) } \\
\hline & & \multirow{2}{*}{$\begin{array}{l}\text { (Other } \\
\text { Forms) }\end{array}$} & \multirow{2}{*}{$\mathrm{C}$} & \multirow{2}{*}{ Mn } & \multirow{2}{*}{$\mathbf{P}$} & \multicolumn{2}{|c|}{$\mathrm{S}$} & \multirow{2}{*}{$\mathrm{Si}$} & \multirow{2}{*}{$\mathrm{Cu}$} \\
\hline & & & & & & Grav & Comb & & \\
\hline $73 \mathrm{c}$ & Cr13 (SAE 420) & $\ldots \ldots$ & 0.310 & 0.330 & 0.018 & & 0.036 & 0.181 & 0.080 \\
\hline $121 d$ & Cr17-Ni11-Ti0.3 (AISI 321) & 1171 & .067 & 1.80 & .019 & & .013 & .54 & .121 \\
\hline $123 \mathrm{c}$ & CR17-Ni11-Nb0.6 (AISI 348) & 1172 & .056 & $1.7_{5}$ & .024 & & .014 & .59 & .103 \\
\hline $133 \mathrm{a}$ & CR13-Mo0.3-S0.3 & 1910 & .120 & 1.03 & .026 & 0.326 & .330 & .412 & .118 \\
\hline $160 \mathrm{~b}$ & Cr19-Ni12-Mo3 & 1155 & .044 & 1.64 & .020 & & .018 & $.50_{9}$ & .172 \\
\hline $166 \mathrm{c}$ & 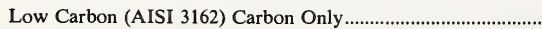 & & .0078 & & & & & & \\
\hline 339 & Cr17-Ni9-Se0.2 (SAE 303Se) & …......... & .052 & 0.738 & .129 & & .013 & .654 & .199 \\
\hline 367 & Cr24-Ni0.3(AISI 446) & 1267 & .093 & .315 & .018 & .................. & .016 & .58 & ........... \\
\hline
\end{tabular}




\begin{tabular}{|c|c|c|c|c|c|c|c|c|c|c|c|}
\hline SRM & $\mathrm{Ni}$ & $\mathrm{Cr}$ & V & Mo & Co & $\mathrm{Ti}$ & $\mathrm{Nb}$ & $\mathrm{Ta}$ & $\mathrm{Pb}$ & $\mathrm{Se}$ & $\mathrm{N}$ \\
\hline $73 c$ & 0.246 & 12.82 & 0.030 & \multirow{5}{*}{$\begin{array}{c}0.091 \\
.165 \\
.22 \\
.294 \\
2.38\end{array}$} & & & & & & & \multirow{2}{*}{0.037} \\
\hline $121 \mathrm{~d}$ & 11.17 & $17.4_{3}$ & ..……......... & & \multirow{3}{*}{$\begin{array}{r}0.10 \\
.12\end{array}$} & \multirow[t]{2}{*}{0.342} & & & & & \\
\hline $123 \mathrm{c}$ & $11.3_{4}$ & $17.4_{0}$ & & & & & \multirow[t]{2}{*}{0.65} & \multirow[t]{2}{*}{$<0.001$} & & & \\
\hline $133 a$ & 0.241 & 12.89 & .026 & & & & & & & & \multirow{2}{*}{$\begin{array}{l}.032 \\
.03_{9}\end{array}$} \\
\hline $160 \mathrm{~b}$ & $12.2_{6}$ & $18.4_{5}$ & .047 & & $.10_{1}$ & & & $\ldots$ & 0.001 & $\ldots$ & \\
\hline \multirow{3}{*}{$\begin{array}{l}166 \mathrm{c} \\
339 \\
367\end{array}$} & & & & & & & & & & & \\
\hline & 8.89 & 17.42 & .058 & 0.248 & .096 & & & & & 0.247 & \multirow[b]{2}{*}{.168} \\
\hline & 0.29 & 24.19 & .08 & & & & & & & & \\
\hline
\end{tabular}

Tool Steels

\begin{tabular}{|c|c|c|c|c|c|c|c|c|}
\hline \multirow{3}{*}{ SRM } & \multirow{3}{*}{ Type } & \multicolumn{7}{|c|}{ Chemical Composition (Nominal Weight Percent) } \\
\hline & & \multirow{2}{*}{$\mathrm{C}$} & \multirow{2}{*}{$\mathrm{Mn}$} & \multirow{2}{*}{$\mathbf{P}$} & \multicolumn{2}{|c|}{$\mathrm{S}$} & \multirow{2}{*}{$\mathrm{Si}$} & \multirow{2}{*}{$\mathrm{Cu}$} \\
\hline & & & & & Grav & Comb & & \\
\hline $50 \mathrm{c}$ & 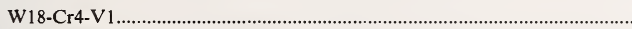 & 0.719 & 0.342 & 0.022 & 0.010 & 0.009 & 0.311 & 0.079 \\
\hline $132 b$ & Mo-W-Cr-V & $.86_{5}$ & .340 & $.01_{3}$ & & .005 & .18 & $.08_{7}$ \\
\hline $134 a$ & Mo8-W2-Cr4-V1 & .808 & .218 & .18 & .007 & .007 & .323 & .101 \\
\hline $153 a$ & Co8-Mo9-W2-Cr4-V2 & .902 & .192 & .023 & .007 & .007 & .270 & .094 \\
\hline
\end{tabular}

\begin{tabular}{|c|c|c|c|c|c|c|c|c|c|}
\hline SRM & $\mathrm{Ni}$ & $\mathrm{Cr}$ & V & Mo & W & Co & $\mathrm{Sn}$ & As & $\mathrm{N}$ \\
\hline $50 \mathrm{c}$ & 0.069 & 4.13 & 1.16 & 0.082 & 18.44 & & 0.018 & 0.022 & 0.012 \\
\hline $132 b$ & .23 & 4.38 & 1.84 & $4.9_{3}$ & $6.2_{8}$ & 0.028 & & & \\
\hline $134 a$ & .088 & 3.67 & 1.25 & 8.35 & 2.00 & & ................ & & ............. \\
\hline $153 a$ & .168 & 3.72 & 2.06 & 8.85 & 1.76 & 8.47 & & & .024 \\
\hline
\end{tabular}

\section{Steels (Granular Form)}

These granular-form SRM's are prepared by a pre-alloyed powder metallurgical process, which generally includes argon atomization and hydrogen annealing. The materials normally are sized between 0.07 to $0.7 \mathrm{~mm}$ to ensure satisfactory homogeneity and are issued in 100-gram units.

\begin{tabular}{|c|c|c|c|c|c|c|c|c|}
\hline \multirow{2}{*}{ SRM } & \multirow{2}{*}{ Type } & \multicolumn{7}{|c|}{$\begin{array}{c}\text { Chemical Composition (Nominal Weight } \\
\text { Percent) }\end{array}$} \\
\hline & & C & Mn & $\mathbf{P}$ & $\mathrm{S}$ & $\mathrm{Si}$ & $\mathrm{Cu}$ & $\mathrm{Ni}$ \\
\hline 163 & Low Alloy, $1.0 \mathrm{Cr}$ & 0.933 & 0.897 & 0.007 & 0.027 & 0.488 & 0.087 & 0.081 \\
\hline $101 \mathrm{f}$ & Stainless (AISI 304L) & .014 & .087 & .008 & .008 & .876 & .030 & 9.96 \\
\hline
\end{tabular}




\begin{tabular}{|c|c|c|c|c|c|c|c|c|c|}
\hline SRM & $\mathrm{Cr}$ & V & Mo & w & Co & $\mathrm{N}$ & As & $\mathrm{Sb}$ & $\mathrm{Ga}$ \\
\hline \multirow{2}{*}{$\begin{array}{l}163 \\
101 f\end{array}$} & \multirow{2}{*}{$\begin{array}{c}0.982 \\
18.49\end{array}$} & & \multirow{2}{*}{$\begin{array}{r}0.029 \\
.007\end{array}$} & & & \multirow{2}{*}{0.007} & & & \\
\hline & & 0.034 & & $(0.0002)$ & 0.088 & & $(0.003)$ & $(0.0009)$ & $(0.004)$ \\
\hline
\end{tabular}

\section{Steels (Solid Form)}

Several groups of SRM's have been prepared to meet the basic needs of the steel industry for analytical control primarily by optical emission and x-ray spectroscopic methods of analysis. Both nominal composition and analytical range SRM's are provided for ingot iron, low-alloy steel, stainless steel, tool steel, and specialty steel.

These SRM's are furnished in various forms. The 400 series is intended for optical emission spectroscopic methods of analysis utilizing the "point-to-point" technique. The 600 series is intended for microchemical methods of analysis such as electron probe microanalysis, spark source mass spectrometric analysis, and laser probe analysis. The 800, 1100, and 1200 series are intended for "point-to-plane" optical emission spectroscopic methods of analysis. The D800 series, and the 1100 and 1200 series also are intended for xray spectroscopic methods of analysis.

Because of the special homogeneity requirements, most of these materials have been prepared by using the most modern techniques of melting, casting, fabrication, and heat treatment to ensure adequate uniformity of composition.

NOTE: Values in parentheses are not certified as they are based on the results from a single laboratory. Values in brackets are not certified but are nominal values obtained from heat analyses. These values are given for additional information on the chemical composition.

\section{Nominal Sizes for Solid Steel SRM's}

400 Series: $5.5 \mathrm{~mm}(7 / 32 \mathrm{in})$ diameter, $102 \mathrm{~mm}(4 \mathrm{in})$ long

600 Series: $3.2 \mathrm{~mm}(1 / 8 \mathrm{in})$ diameter, $51 \mathrm{~mm}(2 \mathrm{in})$ long

800 Series: $13 \mathrm{~mm}(1 / 2 \mathrm{in})$ diameter, $51 \mathrm{~mm}(2 \mathrm{in})$ long

D800 Series: $31 \mathrm{~mm}(1 \mathrm{1} / 4 \mathrm{in})$ diameter, $6.4 \mathrm{~mm}(1 / 4 \mathrm{in})$ thick

1100 and 1200 Series: $31 \mathrm{~mm}(11 / 4 \mathrm{in})$ diameter, $19 \mathrm{~mm}(3 / 4 \mathrm{in})$ thick

\section{Ingot Iron and Low-Alloy Steels}

The preparation of these original spectroscopic SRM's began in about 1944 when the cores remaining after lathe cutting the materials for chip form standards were tested for homogeneity. Those found satisfactory were fabricated to the final shapes and sizes. To meet the urgent need in the mid-1950's for calibration standards for $\mathrm{x}$-ray spectroscopic methods of analysis, portions of the material from five of these SRM's were converted to the applicable disk form. Although entirely satisfactory for conventional spectroscopic methods of analysis, these SRM's generally do not meet the stringent requirements for homogeneity necessary for use with the newer microchemical methods of analysis. These standards will be discontinued when the supply is exhausted. 


\begin{tabular}{|c|c|c|c|c|c|c|c|c|c|c|c|c|c|}
\hline & \multirow[b]{2}{*}{ SRM } & & \multirow[b]{2}{*}{ Type } & \multicolumn{10}{|c|}{ Chemical Composition (Nominal Weight Percent) } \\
\hline & & & & $\mathrm{Mn}$ & $\mathrm{Si}$ & $\mathrm{Cu}$ & $\mathrm{Ni}$ & $\mathrm{Cr}$ & V & Mo & Sn & $\begin{array}{c}\text { A1 } \\
\text { (Total) }\end{array}$ & Other \\
\hline & $803 a$ & D803a & Acid Open Hearth, 0.6C..... & & 0.34 & 0.096 & 0.190 & 0.101 & 0.005 & 0.033 & & & \\
\hline 404a & $4 a$ & ................. & Basic Electric......... & 0.88 & .44 & .050 & .040 & .025 & .002 & .007 & & & \\
\hline 405a & $805 a$ & & Medium Manganese..... & 1.90 & .27 & .032 & .065 & 0.37 & & .005 & & 0.056 & \\
\hline $407 a$ & $807 a$ & D807a & Chromium-Vanadium.. & 0.76 & .29 & .132 & .169 & .92 & .146 & & & & \\
\hline $408 \mathrm{a}$ & $808 \mathrm{a}$ & ................. & Chromium-Nickel........ & .76 & .28 & .10 & 1.20 & .655 & .002 & .065 & & & \\
\hline 409b & $809 b$ & ............... & Nickel ....................................... & .46 & 27 & .104 & 3.29 & .072 & .002 & .009 & 0.012 & & Co 0.025 \\
\hline 413 & ........... & …............ & Acid Open Hearth, 0.4C................... & .67 & .22 & .25 & 0.18 & .055 & .007 & .006 & & & \\
\hline 414 & & ............... & Cr-Mo (SAE 4140) .................. & .67 & .26 & .11 & .080 & .99 & .003 & .32 & .014 & .020 & \\
\hline $417 \mathbf{a}$ & $817 a$ & ….............. & Basic Open Hearth, 0.4C .................. & .78 & & .13 & .062 & .050 & & .013 & .036 & & \\
\hline $418 a$ & & ............... & Cr-Mo (SAE X4130) ......................... & .52 & .27 & .040 & .125 & 1.02 & & .21 & & & \\
\hline $420 \mathrm{a}$ & $820 \mathrm{a}$ & ............... & Ingot Iron & .017 & .......... & .027 & .0092 & 0.0032 & & .0013 & .0017 & .003 & Co .006 \\
\hline & 821 & ................. & $\mathrm{Cr}-\mathrm{W}, 0.9 \mathrm{C}$ & 1.24 & & .080 & .10 & .49 & .012 & .040 & & & W .52 \\
\hline 427 & 827 & …............. & Cr-Mo (SAE 4150) (B only)......... & & & & & & & & & & В .0027 \\
\hline
\end{tabular}

\section{Special Ingot Irons and Low-Alloy Steels}

The planning of the 1100 series SRM's began in late 1952 to meet critical requirements of calibration in the iron and steel industry. Steel for these SRM's was prepared by the most modern melting, casting, and fabrication techniques to provide large quantities of material of the highest possible homogeneity. The materials were fully characterized and included investigations by means of electron probe microanalysis and quantitative metallographic techniques. It was concluded that, for example, SRM's 461 and 463 are sufficiently homogeneous that any present microanalytical technique can be carried out with little chance of inaccuracy caused by inhomogeneity. Details of the metallographic and homogeneity characterization are given in NBS Miscellaneous Publication 260-3 and 260-10, respectively (see inside back cover for ordering instructions).

The 1200 series replaces the 1100 series which has been exhausted and consists of four low alloy steels and an electrolytic iron containing a graded series of 40 elements. Material from the same melts are available in three other forms: chip form, 361-365, for chemical methods of analysis (page 18); rods, 661-665, 3.2 $\mathrm{mm}(1 / 8 \mathrm{in})$ in diameter and $51 \mathrm{~mm}$ ( 2 in) long for microchemical methods of analysis such as electron probe microanalysis, spark source mass spectrometric analysis, and laser probe analysis (see below); and rods (1095-1099), $6.4 \mathrm{~mm}$ (1/4 in) in diameter and $102 \mathrm{~mm}$ (4 in) long for determining gases in metals by vacuum fusion and neutron activation methods of analysis (page 41). The preparation of the 1200 series involved a cooperative effort between Industry and NBS, and represents the first application of the "benchmark" concept to SRM's. With thousands of industrial processes requiring analytical control, demands for SRM's far exceeded the NBS production capacity. An ever widening gap between supplies and demands led to a program to produce essential "benchmark" SRM's to serve as calibration points in measurement systems. While other selected low-alloy steel SRM's will be prepared to augment the 1200 series, this series is expected to be the primary "benchmarks," especially for some 25 trace elements that affect the physical properites of steels.

NOTE: Values in parentheses not certified, based on a single analytical method.

Values in brackets not certified, approximate values from the heat analyses.

†From Gasometric Certificates: SRM's 1095 through 1099.

-Not detected, value given is conservative "Upper Limit" of detection by a specific method of analysis. 


\begin{tabular}{|c|c|c|c|c|c|c|c|c|c|c|c|}
\hline \multirow{2}{*}{\multicolumn{2}{|c|}{ SRM }} & \multirow[b]{2}{*}{ Type } & \multicolumn{9}{|c|}{ Chemical Composition (Nominal Weight Percent) } \\
\hline & & & $\begin{array}{l}\text { (Other } \\
\text { Forms) }\end{array}$ & $\mathrm{C}$ & $\mathrm{Mn}$ & $\mathbf{P}$ & $\mathbf{S}$ & $\mathrm{Si}$ & $\mathrm{Cu}$ & $\mathrm{Ni}$ & $\mathrm{Cr}$ \\
\hline & 1134 & High-Silicon .... & $125 \mathrm{~b}$ & 0.026 & 0.277 & 0.028 & 0.009 & 2.89 & 0.070 & 0.038 & 0.019 \\
\hline & 1135 & High-Silicon ................ & 179 & .027 & .094 & .006 & .026 & 3.19 & .056 & .050 & .022 \\
\hline & 1136 & 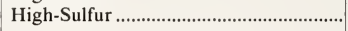 & $129 \mathrm{c}$ & $.11_{3}$ & $.75_{5}$ & .066 & .22 & 0.018 & .014 & .27 & .014 \\
\hline 461 & & Low Alloy A & ..................... & .15 & .36 & .053 & $(.02)$ & .047 & .34 & 1.73 & .13 \\
\hline 462 & & Low Alloy B ........ & ………........... & .40 & .94 & .045 & $(.02)$ & .28 & .20 & 0.70 & .74 \\
\hline 464 & & 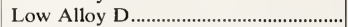 & ………............. & .54 & 1.32 & .017 & $(.02)$ & .48 & .094 & $.13_{\mathrm{s}}$ & .078 \\
\hline 465 & & 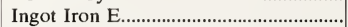 & …………........ & .037 & 0.032 & .008 & (.01) & .029 & .019 & .026 & .004 \\
\hline 466 & 61166 & Ingot Iron F & ………........... & .065 & $.11_{3}$ & .012 & (.01) & .025 & .033 & .051 & .011 \\
\hline 467 & & 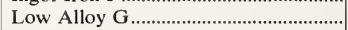 & (................................... & .11 & $.27_{5}$ & .033 & $(.01)$ & .26 & .067 & .088 & .036 \\
\hline 468 & & Low Alloy H...……………………........ & ......................... & .26 & .47 & .023 & $(.02)$ & .075 & .26 & 1.03 & .54 \\
\hline & $1169 \mathrm{a}$ & 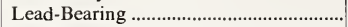 & ………........... & (.1) & $(1.0)$ & $(.07)$ & $(.3)$ & $(.01)$ & $(.1)$ & $(0.05)$ & $(.04)$ \\
\hline & 1222 & 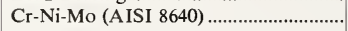 & $139 \mathrm{~b}$ & .43 & 0.78 & .013 & .022 & .24 & .097 & .51 & .48 \\
\hline *661 & $11261 \mathrm{a}$ & AISI 4340. & 361,1095 & $.39_{1}$ & .67 & .016 & .015 & .228 & .042 & 2.00 & $.69_{3}$ \\
\hline *662 & $21262 \mathrm{a}$ & AISI 94B17 (Mod) ……………………....... & 362,1096 & $.16_{1}$ & 1.05 & .044 & .037 & .40 & .51 & 0.60 & .30 \\
\hline *663 & $31263 \mathrm{a}$ & 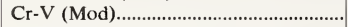 & 363,1097 & $.62_{6}$ & 1.50 & 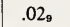 & $.005_{7}$ & .74 & $.09_{8}$ & .32 & 1.31 \\
\hline *664 & $41264 \mathrm{a}$ & High Carbon (Mod) & 364,1098 & .87 & $0.25_{8}$ & .010 & .025 & .067 & .250 & $.14_{2}$ & $0.06_{6}$ \\
\hline *665 & $51265 \mathrm{a}$ & Electrolytic Iron & 365,1099 & .0067 & .0057 & $.002_{5}$ & .0055 & $.008_{0}$ & .0058 & .041 & .007 \\
\hline
\end{tabular}

SRM's $661,662,663,664$, and 665 are sold in a set only as SRM 668 .

\begin{tabular}{|c|c|c|c|c|c|c|c|c|}
\hline \multicolumn{2}{|c|}{ SRM } & B & $\mathrm{Pb}$ & $\mathrm{Ag}$ & $\mathrm{Ge}$ & $\mathrm{O}$ & $\mathrm{N}$ & $\mathrm{H}$ \\
\hline \multicolumn{2}{|l|}{461} & $0.00 O_{2}$ & $(0.003)$ & $\left(0.001_{5}\right)$ & $\left(0.001_{5}\right)$ & $\left(0.02_{0}\right)$ & $\left(0.00_{6}\right)$ & ‥ \\
\hline \multicolumn{2}{|l|}{$\begin{array}{l}462 \\
464\end{array}$} & $.000_{5}$ & .006 & $(<.0002)$ & $\left(.003_{0}\right)$ & $(.006)$ & $\left(.00_{8}\right)$ & 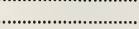 \\
\hline \multicolumn{2}{|l|}{464} & .005 & .020 & $\left(.003_{2}\right)$ & $\left(.001_{5}\right)$ & $(.006)$ & $\left(.00_{7}\right)$ & 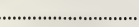 \\
\hline \multicolumn{2}{|c|}{465} & $.000_{1}$ & $(<.0005)$ & $\left(.0002_{5}\right)$ & $\left(.003_{5}\right)$ & $(.003)$ & $\left(.00_{5}\right)$ & 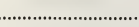 \\
\hline \multirow{2}{*}{\multicolumn{2}{|c|}{$\begin{array}{l}4661166 \\
467\end{array}$}} & $\left(.000_{2}\right)$ & $\left(.001_{3}\right)$ & $\left(.0004_{5}\right)$ & $\left(.003_{0}\right)$ & $(.005)$ & $\left(.00_{6}\right)$ & ................................. \\
\hline & & $\left(.000_{2}\right)$ & $.000_{6}$ & $\left(.004_{0}\right)$ & $\left(.003_{0}\right)$ & $(.004)$ & $\left(.00_{4}\right)$ & 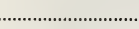 \\
\hline \multirow{3}{*}{468} & \multirow{3}{*}{$\begin{array}{l}1169 \mathrm{a} \\
1222\end{array}$} & .009 & $(<.0005)$ & $(<.0005)$ & $\left(.001_{0}\right)$ & $(.004)$ & $\left(.00_{6}\right)$ & $\ldots$ \\
\hline & & & .29 & & & & & \\
\hline & & & & & 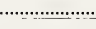 & $(-1)$ & $(.007)$ & 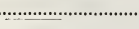 \\
\hline *661 & $1261 \mathrm{a}$ & .0005 & $.00002_{5}$ & .0004 & {$[.006]$} & $(.0009) \dagger$ & $(.0037) \dagger$ & {$[<0.0005]^{\dagger}$} \\
\hline *662 & $1262 \mathrm{a}$ & .0025 & $.0004_{3}$ & .0011 & {$[.002]$} & $(.00107) \dagger$ & $(.00404) \dagger$ & {$[<.0005]^{\dagger}$} \\
\hline *663 & $1263 \mathrm{a}$ & $.0009_{1}$ & .0022 & $(.0037)$ & {$[.010]$} & $(.00066) \dagger$ & $(.0041) \dagger$ & {$[<.0005] \dagger$} \\
\hline *664 & $1264 \mathrm{a}$ & .011 & .024 & $(.00002)$ & {$[.003]$} & $(.0010) \dagger$ & $(.0032) \dagger$ & {$[<.0005] \dagger$} \\
\hline *665 & $1265 a$ & .00013 & $.00001_{5}$ & $(.000002)$ & $(\sim .0014)$ & $(.0063) \dagger$ & $(\sim .0011)$ & $(\sim .0001) \dagger$ \\
\hline
\end{tabular}

*SRM's $661,662,663,664$, and 665 are sold in a set only as SRM 668.

\begin{tabular}{|c|c|c|c|c|c|c|c|c|c|c|c|}
\hline SRM & V & Mo & w & Co & $\mathrm{Ti}$ & As & Sn & $\begin{array}{c}\mathrm{Al} \\
\text { (Total) }\end{array}$ & $\mathrm{Nb}$ & $\mathrm{Ta}$ & $\mathrm{Zr}$ \\
\hline 1134 & & 0.008 & & & & & 0.003 & 0.329 & & & \\
\hline 1145 & $<0.01$ & .014 & .......... & & ........... & 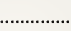 & .004 & .0028 & $\cdots \cdots$ & $\ldots \ldots \ldots \ldots$ & ..................... \\
\hline 1136 & .012 & .002 & & & & & & & & & 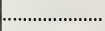 \\
\hline 461 & .024 & .30 & 0.012 & 0.26 & $(0.01)$ & 0.028 & .022 & .005 & 0.011 & 0.002 & $(<0.005)$ \\
\hline 462 & .058 & .080 & .053 & .11 & .037 & .046 & .066 & $.02_{3}$ & .096 & .036 & .063 \\
\hline 464 & $.29 \mathrm{~s}$ & .029 & .022 & $.02_{8}$ & .004 & .018 & .043 & .005 & .037 & .069 & .010 \\
\hline 465 & .002 & .005 & $(.001)$ & .008 & .20 & .010 & .001 & .19 & $(.001)$ & .001 & $(.002)$ \\
\hline $466 \quad 1166$ & .007 & .011 & $(.006)$ & $.04_{6}$ & .057 & .014 & .005 & $.01_{5}$ & .005 & .002 & $(<.005)$ \\
\hline 467 & .041 & .021 & .20 & .074 & .26 & .14 & .10 & .16 & .29 & .23 & .094 \\
\hline 468 & .17 & .20 & .077 & .16 & .011 & .008 & .009 & $.04_{2}$ & .006 & .005 & $(<.005)$ \\
\hline
\end{tabular}




\begin{tabular}{|c|c|c|c|c|c|c|c|c|c|c|c|}
\hline SRM & V & Mo & W & Co & $\mathrm{Ti}$ & As & Sn & $\begin{array}{c}\text { A1 } \\
\text { (Total) }\end{array}$ & $\mathrm{Nb}$ & Ta & $\mathrm{Zr}$ \\
\hline 1222 & .005 & .18 & 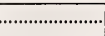 & $(.016)$ & $(.002)$ & $\ldots \ldots \ldots \ldots$ & 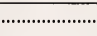 & $(.038)$ & $(.002)$ & os os os & 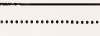 \\
\hline *661 1261a & .011 & .19 & .017 & .032 & .020 & .017 & .010 & $.02_{1}$ & .022 & .021 & .009 \\
\hline${ }^{*} 6621262 \mathrm{a}$ & $.04_{1}$ & .07 。 & .20 & .30 & .085 & $.09_{5}$ & .016 & $.09_{s}$ & .30 & .21 & .20 \\
\hline${ }^{*} 663 \mathrm{1263a}$ & .31 & .030 & .046 & .048 & .050 & .010 & $.10_{4}$ & .24 & .049 & $(.053)$ & .050 \\
\hline *664 1264a & $.10_{6}$ & .49 & $.10_{2}$ & .15 & .24 & $.05_{2}$ & .008 & $(.0080)$ & $.15_{7}$ & .11 & .069 \\
\hline${ }^{*} 6651265 \mathrm{a}$ & .0006 & .0050 & $(.0004)$ & .007 。 & .0006 & $(.0002)$ & $(.0002)$ & $(.0007)$ & & $(<.00005)$ & $(<.00001)$ \\
\hline
\end{tabular}

\begin{tabular}{|c|c|c|c|c|c|c|}
\hline SRM & $\mathrm{Sb}$ & $\mathrm{Bi}$ & $\mathrm{Ca}$ & $\mathrm{Mg}$ & $\mathrm{Se}$ & $\mathrm{Te}$ \\
\hline 1222 & & & & & & \\
\hline *661 1261a & 0.0042 & 0.0004 & $0.00002_{8}$ & 0.00018 & 0.004 & 0.0006 \\
\hline *662 1262a & .012 o & $(.002)$ & .00014 & .00062 & $(.0012)$ & .0011 \\
\hline *663 1263a & .002 & $(.0008)$ & .00013 & .00049 & $(.00016)$ & .0009 \\
\hline *664 1264a & .034 & $(.0009)$ & .00004 & .00015 & $(.00021)$ & .00018 \\
\hline *665 1265a & $-(<.00005)$ & $-(<.00001)$ & $-(<.00001)$ & $-(<.00002)$ & $-(<.00001)$ & $-(<.00001)$ \\
\hline
\end{tabular}

\begin{tabular}{|c|c|c|c|c|c|c|c|c|}
\hline SRM & $\mathrm{Zn}$ & $\mathrm{Au}$ & $\mathrm{Ce}$ & $\mathrm{Hf}$ & $\mathrm{La}$ & $\mathrm{Nd}$ & $\operatorname{Pr}$ & $\mathrm{Fe}$ \\
\hline *661 1261a & $(0.0001)$ & $(<0.00005)$ & 0.0014 & $(0.0002)$ & 0.0004 & 0.0002 & $(0.00014)$ & (95.6) \\
\hline${ }^{*} 662 \quad 1262 \mathrm{a}$ & $(.0005)$ & $(<.00005)$ & .0015 & $(.0003)$ & .0004 & $.0006_{4}$ & $(.00012)$ & (95.3) \\
\hline${ }^{*} 663 \quad 1263 a$ & $(.0004)$ & .0005 & .0014 & $(.0005)$ & .0006 & $.0006_{0}$ & $(.00018)$ & (94.4) \\
\hline *664 1264a & {$[.001]$} & .0001 & $.0002_{2}$ & $(.0013)$ & .00007 & .00007 & $(.00003)$ & (96.7) \\
\hline *665 1265a & $(<.0001)$ & $-(<.000002)$ & $-(<.000005)$ & $-(<.00002)$ & $-(<.000005)$ & $-(<.000005)$ & $-(<.000005)$ & (99.9) \\
\hline
\end{tabular}

*SRM's $661,662,663,664$, and 665 are sold in a set only as SRM 668.

\section{Stainless Steel}

Three groups of stainless steel SRM's designed primarily for calibration in spectroscopic methods of analysis are available.

Groups I and II have been extensively tested for homogeneity and found satisfactory for application in conventional spectroscopic methods of analysis. Neither group, however, has been tested for microanalytical methods and their use in these applications is not recommended.

Group III are for the "point-to-plane" technique of emission spectoscopy and for x-ray spectroscopy. They were prepared by melting, casting, and fabrication techniques known to produce material of high homogeneity.

(Values in parentheses are not certified, but are given for additional information only.)

GROUP I

\begin{tabular}{|c|c|c|c|c|c|c|c|c|c|c|}
\hline \multirow{2}{*}{ SRM } & \multirow{2}{*}{ Name } & \multicolumn{9}{|c|}{ Chemical Composition (Nominal Weight Percent) } \\
\hline & & $\mathbf{M n}$ & $\mathrm{Si}$ & $\mathrm{Cu}$ & $\mathrm{Ni}$ & $\mathrm{Cr}$ & V & Mo & W & Co \\
\hline 442 & 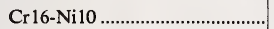 & 2.88 & $(0.09)$ & 0.11 & 9.9 & 16.1 & 0.032 & 0.12 & $(0.08)$ & 0.13 \\
\hline 443 & $\mathrm{Cr} 18.5-\mathrm{Ni9} .5 \ldots \ldots \ldots \ldots \ldots \ldots \ldots$ & 3.38 & $(.15)$ & .14 & 9.4 & 18.5 & .064 & .12 & $(.09)$ & .12 \\
\hline 444 & $\mathrm{Cr} 20.5-\mathrm{Ni} 10$ & 4.62 & $(.65)$ & .24 & 10.1 & 20.5 & .12 & .23 & $(.17)$ & .22 \\
\hline
\end{tabular}




\begin{tabular}{|c|c|c|c|c|c|c|c|c|}
\hline SRM & $\mathrm{Ti}$ & Sn & $\mathrm{Nb}$ & $\mathrm{Ta}$ & B & $\mathrm{Pb}$ & $\mathrm{Zr}$ & $Z_{n}$ \\
\hline 442 & 0.002 & 0.0035 & 0.032 & $(0.0006)$ & 0.0005 & 0.0017 & $(0.004)$ & $(0.003)$ \\
\hline 443 & .003 & .006 & .056 & $(.0008)$ & .0012 & .0025 & & $(.005)$ \\
\hline 444 & .019 & .014 & .20 & $(.004)$ & .0033 & .0037 & $(.011)$ & $(.004)$ \\
\hline
\end{tabular}

GROUP II

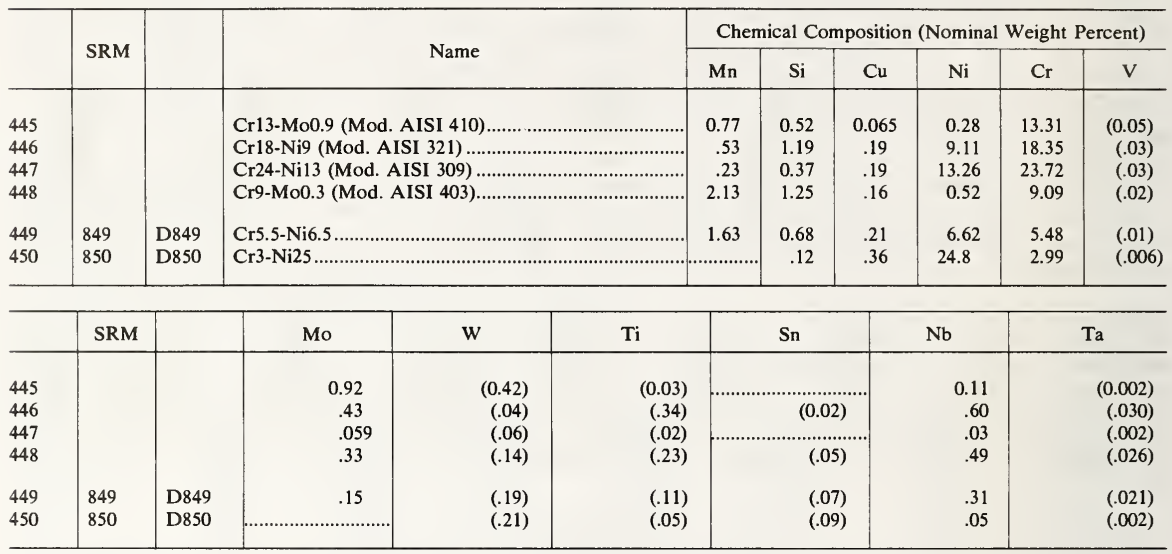

GROUP III

\begin{tabular}{|c|c|c|c|c|c|c|c|c|c|c|}
\hline \multirow[b]{2}{*}{ SRM } & \multirow[b]{2}{*}{ Name } & \multicolumn{9}{|c|}{ Chemical Composition (Nominal Weight Percent) } \\
\hline & & $\begin{array}{l}\text { Other } \\
\text { Forms }\end{array}$ & $\mathrm{C}$ & Mn & $\mathbf{P}$ & $\mathbf{s}$ & $\mathrm{Si}$ & $\mathrm{Cu}$ & $\mathrm{Ni}$ & $\mathrm{Cr}$ \\
\hline $1151 \mathrm{a}$ & Cr22-Ni7-IN PREP. & & & & & & & & & \\
\hline C1151 & 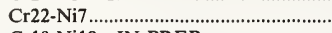 & ……........... & 0.039 & 2.50 & 0.017 & 0.38 & 0.38 & 0.418 & 7.29 & 22.70 \\
\hline $\begin{array}{l}1152 a \\
\mathrm{C} 1152\end{array}$ & Cr18-Ni10-IN PREP ................. & & & & & & & & & \\
\hline $\begin{array}{l}\text { C1152 } \\
1153 a\end{array}$ & $\begin{array}{l}\text { Cr18-Ni10 } \\
\text { Cr16Ni8-IN PREP }\end{array}$ & . & .148 & 0.96 & .021 & .0064 & .80 & .102 & 10.88 & 17.81 \\
\hline C1153 & Cr16-Ni8 . & 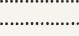 & .264 & .50 & .030 & .018 & 1.07 & .23 & 8.77 & 16.69 \\
\hline $1154 \mathrm{a}$ & Cr19-Ni12-IN PREP. & & & & & & & & & \\
\hline C1154 & Cr19-Ni12 .................................... & & .086 & 1.42 & .06 & .053 & 0.50 & .40 & 12.92 & 19.06 \\
\hline 1155 & Cr18-Ni12-Mo2 (AISI 316)................... & $160 \mathrm{~b}$ & .046 & 1.63 & .020 & .018 & .50 & .169 & 12.18 & 18.45 \\
\hline $1170 \mathrm{~b}$ & Selenium-Bearing ..................................... & & $(.052)$ & $(0.738)$ & $(.129)$ & $(.013)$ & $(.654)$ & (.199) & $(8.89)$ & (17.42) \\
\hline 1171 & Cr17-Nill-Ti0.3...................................... & $121 \mathrm{~d}$ & .067 & 1.80 & .018 & $.01_{3}$ & .54 & .121 & 11.2 & 17.4 \\
\hline 1172 & 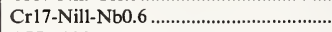 & $123 \mathrm{c}$ & .056 & 1.76 & .025 & $.01_{4}$ & .59 & $.10_{5}$ & $11.3_{5}$ & 17.4 。 \\
\hline 1267 & 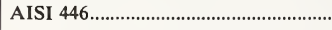 & 367 & .093 & 0.315 & .018 & .015 & .58 & ................ & 0.29 & 24.24 \\
\hline
\end{tabular}




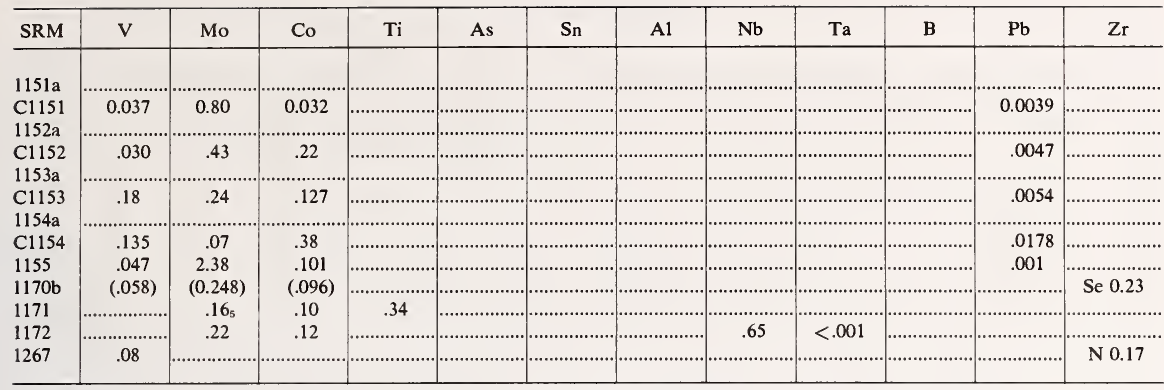

SRM's 1156, Maraging Steel, and 1158, High-Nickel Steel (Invar), are designed primarily for use in optical emission and $\mathrm{x}$-ray spectrometric methods of analysis.

SRM 1156 derives its name from the formation of martensite on age hardening. Alloys of this type are used extensively in submarines, missiles, and aircraft.

SRM 1158 has good impact toughness down to $-269^{\circ} \mathrm{C}$ and has an extremely low coefficient of expansion between -253 and $203^{\circ} \mathrm{C}$. These properties make this material very useful for cryogenic application. SRM 1158 also serves as a "benchmark" for the production control of ferronickel (40Ni-60Fe) alloys.

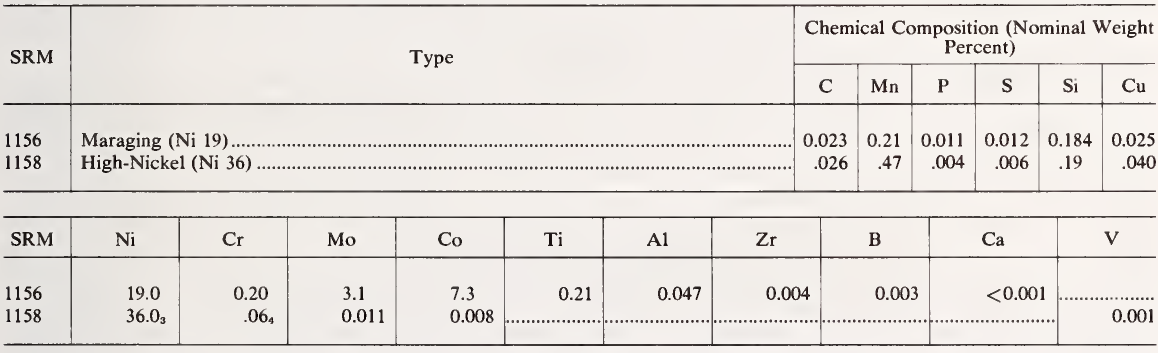

High-Temperoture Alloys (Solid Form)

High-temperature alloy SRM's were prepared to meet the critical needs of industry, particularly the aerospace industry, and government agencies. These SRM's are useful in instrument calibration, primarily for x-ray and optical emission spectroscopic methods of analysis. 


\begin{tabular}{|c|c|c|c|c|c|c|c|}
\hline \multirow{2}{*}{ SRM } & \multirow{2}{*}{ Type } & \multicolumn{6}{|c|}{ Chemical Composition (Nominal Weight Percent) } \\
\hline & & C & Mn & $\mathbf{P}$ & $\mathrm{s}$ & $\mathrm{Si}$ & $\mathrm{Cu}$ \\
\hline 1198 & Incoloy 901 & $(0.048)$ & $(0.49)$ & $(0.006)$ & $(0.002)$ & $(0.38)$ & $(0.012)$ \\
\hline 1199 & L 605 & $(.14)$ & 1.42 & $(.005)$ & & .83 & \\
\hline 1200 & S 816 & (.40) & 1.34 & $(.015)$ & & .86 & $\ldots$ \\
\hline 1201 & Hastelloy X... & (.039) & & $(.008)$ & & (.54) & \\
\hline $1206-2$ & (1) & $.21_{7}$ & 0.030 & $(.004)$ & .006 & .218 & .040 \\
\hline $1207-1$ & Waspaloy(1) & .043 & .34 & .005 & .009 & $.47_{2}$ & .026 \\
\hline $1207-2$ & Waspaloy $(2)$ & .083 & .295 & .005 & .009 & $.61_{5}$ & .033 \\
\hline $1208-1$ & Inco 718(1) & .046 & $.38_{5}$ & .003 & $.01_{1}$ & $.43_{4}$ & $.14_{7}$ \\
\hline $1208-2$ & Inco 718(2) & .022 & .23 。 & .003 & .007 & $.08_{3}$ & .077 \\
\hline
\end{tabular}

\begin{tabular}{|c|c|c|c|c|c|c|c|c|c|c|c|c|}
\hline SRM & $\mathrm{Ni}$ & $\mathrm{Cr}$ & Mo & Co & $\mathrm{Ti}$ & Al & $\mathrm{Nb}$ & $\mathrm{Ta}$ & $\mathrm{Fe}$ & W & B & $\mathrm{Zr}$ \\
\hline 1198 & 40.1 & 12.9 & $6.0_{8}$ & 0.70 & 2.59 & 0.24 & $(<0.02)$ & & 36.2 & $(0.2)$ & $(0.0064)$ & $(0.014)$ \\
\hline 1199 & 10.2 & 19.9 & $(<0.02)$ & 51.6 & $(<0.01)$ & ...................... & $(<.02)$ & & $0.6_{5}$ & 15.4 & & \\
\hline 1200 & 20.0 & 19.9 & $4.0^{\circ}$ & 42.0 & $(.03)$ & ……............ & $3.1_{8}$ & 1.08 & 3.19 & $3.8_{8}$ & & \\
\hline 1201 & 45.7 & 20.7 & $9.1_{8}$ & 0.56 & $(<.01)$ & ……............. & $(<.02)$ & ...................... & 23.2 & (0.15) & & \\
\hline $1206-2$ & 53.3 & 19.7 & $10.3_{0}$ & $11.5_{\mathrm{s}}$ & $2.9_{4}$ & $1.7_{4}$ & & & 0.46 & & & ................ \\
\hline $1207-1$ & 56.1 & 18.88 & 4.50 & $13.0_{5}$ & 3.09 & 1.26 & . & & 2.22 & & .................. & $\ldots \ldots \ldots$ \\
\hline $1207-2$ & 55.7 & $19.4_{4}$ & 4.34 & $13.5_{0}$ & 2.54 & $1.3 \mathrm{~g}$ & & & 2.09 & & & \\
\hline $1208-1$ & 51.9 & 17.5 & $3.2_{4}$ & 0.82 & 0.46 & $(0.15)$ & $5.3_{8}$ & $(0.012)$ & 19.2 & & ……………....... & ………......... \\
\hline $1208-2$ & 51.5 & 17.4 & 3.13 & .76 & $(.85)$ & $(.85)$ & $4.9_{8}$ & $(.012)$ & 19.8 & …………...... & ...………… & ................... \\
\hline
\end{tabular}

Tool Steels

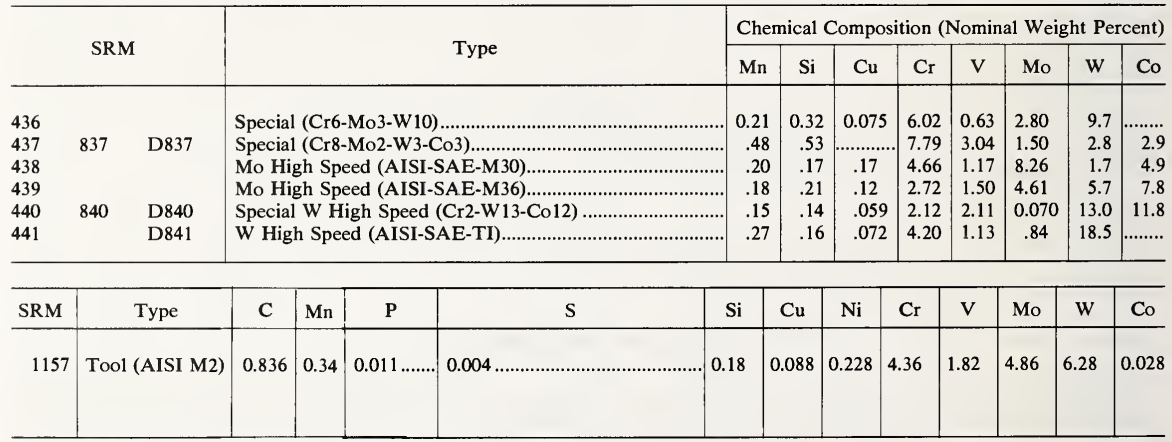


These SRM's provide standards of known chemical composition primarily for checking chemical methods of analysis for the major constituents and for selected minor elements covered by ASTM specifications. They are furnished as fine powders (usually $<0.1 \mathrm{~mm}$ ). These SRM's are finding increased use in calibration with instrumental methods of analysis.

\begin{tabular}{|c|c|c|c|c|c|c|c|c|c|}
\hline \multirow[b]{2}{*}{ SRM } & \multirow[b]{2}{*}{ Type } & \multicolumn{8}{|c|}{ Chemical Composition (Nominal Weight Percent) } \\
\hline & & $\begin{array}{c}\text { Ut/ } \\
\text { Unit } \\
\text { (grams) }\end{array}$ & $\mathrm{C}$ & $\mathrm{Mn}$ & $\mathbf{P}$ & $\mathbf{S}$ & Si & $\mathrm{Cu}$ & $\mathrm{Ni}$ \\
\hline $57 \mathrm{a}$ & Refined Silicon IN PREP.... & & & & & & & & \\
\hline $58 \mathrm{a}$ & Ferrosilicon $(73 \%$ Si) & 75 & 0.014 & 0.16 & 0.009 & $<0.002$ & 73.20 & 0.024 & 0.012 \\
\hline $59 a$ & Ferrosilicon $(50 \% \mathrm{Si})$ & 50 & .04 & .76 & .016 & & 48.2 & .05 & .03 \\
\hline 195 & 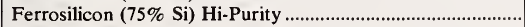 & 75 & .034 & .17 & .02 & $<.002$ & 75.3 & .047 & .032 \\
\hline $64 \mathrm{c}$ & Ferrochromium (HC) & 100 & 4.68 & .16 & .020 & .067 & 1.22 & .005 & .43 \\
\hline 196 & Ferrochromium (LC) & 100 & 0.035 & .28 & & & 0.38 & & \\
\hline 71 & 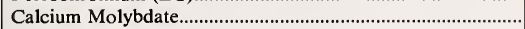 & 60 & & & & & & & \\
\hline 90 & Ferrophosphorus & 75 & & …... & 26.2 & & ............. & & \\
\hline 340 & Ferroniobium & 100 & .060 & 1.71 & 0.035 & & 4.39 & & ............... \\
\hline $68 \mathrm{c}$ & Ferromanganese $(\mathrm{HC})$ & 100 & 6.72 & 80.04 & .19 & .008 & 0.225 & & \\
\hline
\end{tabular}

\begin{tabular}{|c|c|c|c|c|c|c|c|c|c|c|c|c|c|c|}
\hline SRM & $\mathrm{Cr}$ & V & Mo & $\mathrm{Ti}$ & Al & $\mathrm{Nb}$ & $\mathrm{Zr}$ & $\mathrm{Ca}$ & $\mathrm{Mg}$ & $\mathrm{Fe}$ & B & $\mathbf{N}$ & Co & As \\
\hline \multicolumn{15}{|l|}{$57 \mathrm{a}$} \\
\hline $58 \mathrm{a}$ & 0.020 & $(0.002)$ & $(0.01)$ & 0.051 & \multirow{4}{*}{$\begin{array}{c}0.95 \\
.35 \\
(.05)\end{array}$} & & 0.002 & \multirow{3}{*}{0.04} & & \multirow{5}{*}{$\begin{array}{l}25.22 \\
50.0 \\
23.6 \\
24.98\end{array}$} & \multirow{4}{*}{$\begin{array}{l}0.0010 \\
.06 \\
.001\end{array}$} & \multirow{2}{*}{ 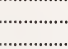 } & \multirow[t]{2}{*}{$<0.01$} & \multirow{2}{*}{ ……...................... } \\
\hline \multirow{2}{*}{$\begin{array}{l}59 a \\
195\end{array}$} & .08 & & & & & & & & & & & & & \\
\hline & .047 & & $(.01)$ & .037 & & $\ldots \ldots \ldots$ & $(<.02)$ & & & & & \multirow{3}{*}{0.045} & \multirow{3}{*}{$\begin{array}{c}<.01 \\
\quad .051\end{array}$} & $\ldots+\ldots$ \\
\hline $64 c$ & 68.00 & .15 & & .02 & & & & & ............ & & & & & $\ldots \ldots$ \\
\hline \multirow{5}{*}{$\begin{array}{l}196 \\
71 \\
90 \\
340 \\
68 c\end{array}$} & 70.87 & .12 & & & & & & & & & & & & \\
\hline & . & 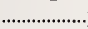 & \multirow{2}{*}{35.3} & \multirow[t]{2}{*}{.06} & & & & & & \multirow[t]{2}{*}{1.92} & & \multirow{2}{*}{ ……........... } & \multirow{2}{*}{ 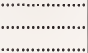 } & \multirow{3}{*}{$\ldots$} \\
\hline & & & & & & & & & & & & & & \\
\hline & & & ...................... & .89 & 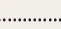 & 57.51 & Ta 3.73 & & & & & & ...…………. & \\
\hline & 0.074 & $\ldots$ & .................... & 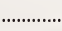 & $\ldots$ & & & ............ & .................. & 12.3 & .......... & $\ldots \ldots . .$. & 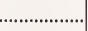 & 0.021 \\
\hline
\end{tabular}

\section{Cast Irons (Chip Form)}

This group of cast iron SRM's is similar to the chip-form steels and was prepared for use in checking chemical methods in the cast iron industry. These SRM's are furnished in 150-g units (unless otherwise noted) and in the form of chips; usually sized between 0.7 to $1.2 \mathrm{~mm}$. They are prepared by lathe cutting of chips with a multiple-tooth cutting tool from thin-wall cylindrical castings especially made for the purpose. Supplied with each SRM is a Certificate of Analysis listing the chemical composition determined at NBS and other laboratories that cooperated in the certification of the SRM's. For SRM 365, Electrolytic Iron, the Certificate provides information on these additional elements: $\mathrm{W}, \mathrm{Nb}, \mathrm{Ag}, \mathrm{Zn}, \mathrm{Ge}, \mathrm{O}, \mathrm{H}, \mathrm{Ta}, \mathrm{Nd}$, $\mathrm{Zr}, \mathrm{Sb}, \mathrm{Bi}, \mathrm{Ca}, \mathrm{Mg}$, Se, Te, Ce, La, Pr, Au, Hf, and Fe.

(Values in parentheses are not certified, but are given for information only.) 


\begin{tabular}{|c|c|c|c|c|c|c|c|c|c|}
\hline \multirow{3}{*}{ SRM } & \multirow{3}{*}{ Type } & \multicolumn{8}{|c|}{ Chemical Composition (Nominal Weight Percent) } \\
\hline & & \multicolumn{2}{|c|}{$\mathrm{C}$} & \multirow[b]{2}{*}{ Mn } & \multirow[b]{2}{*}{$\mathbf{P}$} & \multicolumn{2}{|c|}{ s } & \multirow[b]{2}{*}{$\mathrm{Si}$} & \multirow[b]{2}{*}{$\mathrm{Cu}$} \\
\hline & & Total & $\begin{array}{l}\text { Gra- } \\
\text { phitic }\end{array}$ & & & Grav & Comb & & \\
\hline 3d & White $(110 \mathrm{~g}) \ldots .$. & 2.54 & & 0.40 & 0.025 & & 0.052 & 1.31 & 0.043 \\
\hline $4 \mathrm{k}$ & 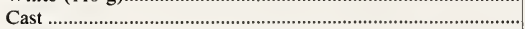 & $3.2_{2}$ & $2.6_{5}$ & $.82_{5}$ & .149 & ........... & .043 & 1.33 & $.24_{3}$ \\
\hline $5 \mathrm{~L}$ & 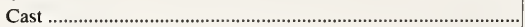 & 2.59 & 1.99 & .68 & .280 & ................ & .123 & 1.83 & 1.01 \\
\hline $6 \mathrm{~g}$ & 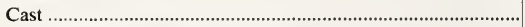 & 2.85 & 2.01 & 1.05 & .557 & & .124 & 1.05 & 0.502 \\
\hline $7 \mathrm{~g}$ & Cast (High Phosphorus) & 2.69 & 2.59 & 0.612 & 0.794 & 0.061 & 0.060 & 2.41 & 0.128 \\
\hline $82 \mathrm{~b}$ & Cast $(\mathrm{Ni}-\mathrm{Cr}) \ldots \ldots \ldots \ldots . . . .$. & 2.85 & 2.37 & .745 & .025 & & .007 & 2.10 & .038 \\
\hline $107 \mathrm{~b}$ & Cast $(\mathrm{Ni}-\mathrm{Cr}-\mathrm{Mo}) \ldots \ldots$ & 2.75 & 1.87 & .510 & .058 & .067 & .067 & 1.35 & .235 \\
\hline $115 \mathrm{a}$ & Cast $(\mathrm{Cu}-\mathrm{Ni}-\mathrm{Cr}) \ldots$ & 2.62 & 1.96 & 1.00 & .086 & .064 & .065 & 2.13 & 5.52 \\
\hline $122 \mathrm{~g}$ & 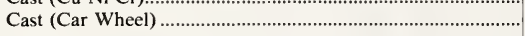 & 3.43 & 2.76 & 0.540 & .315 &........ & .074 & 0.517 & 0.030 \\
\hline 341 & Ductile ............ & 1.81 & 1.23 & .92 & .024 & .007 & .007 & 2.44 & .152 \\
\hline $342 \mathrm{a}$ & 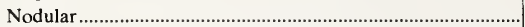 & 1.86 & 1.38 & .275 & .018 & & .006 & 2.73 & .14 \\
\hline 365 & 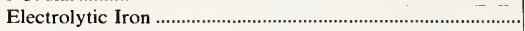 & 0.0068 & .. & .0056 & .0025 & ............. & .0056 & 0.0080 & .0058 \\
\hline
\end{tabular}

\begin{tabular}{|c|c|c|c|c|c|c|c|c|c|c|c|c|}
\hline SRM & $\mathrm{Ni}$ & $\mathrm{Cr}$ & V & Mo & Co & $\mathrm{Ti}$ & As & Sn & Al (total) & $\mathrm{Mg}$ & $\mathrm{N}$ & $\mathrm{Fe}$ \\
\hline $3 d$ & 0.025 & 0.03 & $(0.002)$ & $(0.007)$ & ……….... & $(0.003)$ & & & & & & \\
\hline $4 \mathrm{k}$ & .042 & .116 & .024 & .040 & …………..... & $(.03)$ & $(.03)$ & $(0.004)$ & $(0.004)$ & ...…….......... & \multirow{5}{*}{$\begin{array}{c}(0.0016) \\
.006 \\
.005 \\
.004\end{array}$} & ............ \\
\hline $5 \mathrm{~L}$ & .086 & .15 & .036 & .020 & …………….... & .05 & $<.005$ & ....................... & 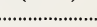 & …………....... & & ................... \\
\hline $6 \mathrm{~g}$ & .135 & .370 & .056 & .035 & ․․․․……….... & .059 & .042 & ……………... & 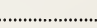 & ․․………...... & & ...……........ \\
\hline $7 g$ & .120 & .048 & .010 & .012 & & .044 & .014 & & ....................... & $\ldots$ & & ........................ \\
\hline $82 \mathrm{~b}$ & 1.22 & .333 & .027 & .002 & & .027 & & & & & & \\
\hline $107 \mathrm{~b}$ & 2.12 & .560 & .008 & .750 & ……............. & .016 & & & & ................. & \multirow[t]{2}{*}{$(.008)$} & ............ \\
\hline $115 a$ & 14.49 & 1.98 & .014 & .050 & …………..... & .020 & & & & & & ............ \\
\hline $122 \mathrm{~g}$ & 0.030 & 0.050 & .038 & $(.003)$ & 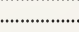 & .034 & . & $\ldots \ldots \ldots \ldots$ & $(.003)$ & & in & $\ldots \ldots$ \\
\hline 341 & 20.32 & 1.98 & .012 & .010 & & .018 & & & & \multirow{3}{*}{$\begin{array}{r}0.068 \\
.069 \\
\mathrm{~N} .001\end{array}$} & & \\
\hline $342 a$ & 0.06 & 0.034 & & & & .020 & & & & & & \\
\hline 365 & .041 & $.007_{2}$ & .0006 & .0050 & $0.007_{2}$ & .0006 & $(.0002)$ & $(.0002)$ & $(.0007)$ & & $\mathrm{Pb} .00002$ & 99.90 \\
\hline
\end{tabular}

\section{Cast Steels, White Cast Irons, Ductile Irons and Blasł Furnace Irons (Solid Form)}

These chill-cast SRM's were prepared for use in analytical control of cast steels and cast irons by rapid instrumental methods. Although employed in x-ray spectroscopic analysis, they are particularly useful for calibrating vacuum optical emission spectrometers because they permit the determination of carbon, phosphorus, and sulfur in addition to the metallic elements.

The "benchmark" concept was used in preparing three new white irons $(1145,1146$, and 1150$)$ with compositions tailored to provide low, nominal, and high values for elements normally specified in cast iron materials, as well as most malleable, ductile, and grey irons. A concentration range for a number of trace elements of interest was provided to enhance the utility of the standards. The planning, preparation, homogeneity testing, and analysis of these SRM's were done through a cooperative Industry-ASTM-NBS program.

These SRM's are chill-cast sections. Details of the preparation and intended use of the SRM's are given in the NBS Miscellaneous Publication 260-1. (See inside back cover for ordering instructions.)

(Values in parentheses are not certified, but are given for information only.) 


\begin{tabular}{|c|c|c|c|c|c|c|c|c|c|}
\hline \multirow{2}{*}{ SRM } & \multirow{2}{*}{ Type } & \multicolumn{8}{|c|}{ Chemical Composition (Nominal Weight Percent) } \\
\hline & & $\mathrm{C}$ & Mn & $\mathbf{P}$ & $\mathrm{S}$ & $\mathrm{Si}$ & $\mathrm{Cu}$ & $\mathrm{Ni}$ & $\mathrm{Cr}$ \\
\hline $1138 \mathrm{a}$ & Cast Steel (No. 1).... & $0.11_{8}$ & 0.35 & 0.035 & 0.056 & 0.25 & 0.09 & 0.10 & 0.13 \\
\hline $1139 \mathrm{a}$ & Cast Steel (No. 2) & .79 。 & .92 & .012 & .013 & .80 & .47 & .98 & $2.1_{8}$ \\
\hline $1143 \mathrm{a}$ & 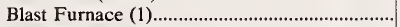 & 4.08 & $.29_{6}$ & $.16_{4}$ & .067 & 1.60 & $.13_{\mathrm{s}}$ & $.11_{6}$ & $0.16_{3}$ \\
\hline $1144 \mathrm{a}$ & 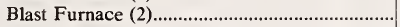 & 4.32 & 1.23 & $.08_{4}$ & .083 & $0.18_{2}$ & $.09_{1}$ & $.06_{3}$ & .029 \\
\hline 1145 & White Cast Iron & 2.85 & 0.040 & .24 & .21 & .29 & .52 & .59 & .67 \\
\hline 1146 & White Cast Iron & 2.01 & 1.64 & .55 & .022 & 3.68 & 1.49 & 3.01 & 2.56 \\
\hline 1150 & White Cast Iron & 3.48 & 0.81 & .063 & .070 & 1.24 & 0.092 & 0.074 & 0.95 \\
\hline
\end{tabular}

\begin{tabular}{|c|c|c|c|c|c|c|c|}
\hline SRM & V & Mo & $\mathrm{Ti}$ & As & Al & $\mathrm{Te}$ & Co \\
\hline $1138 \mathrm{a}$ & 0.020 。 & 0.05 & $(0.0012)$ & $(<0.005)$ & $(0.067)$ & & \\
\hline $1139 a$ & .26 & .51 & $(.004)$ & $(<.005)$ & $(.13)$ & & ............................ \\
\hline $1143 a$ & .018 & (.004) & $.08_{7}$ & $(.003)$ & $(.008)$ & $0.01_{6}$ & 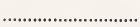 \\
\hline $1144 a$ & $.02_{5}$ & $(.007)$ & .32 & $(.004)$ & $(<.005)$ & $.02_{2}$ & ․․․․․․………...... \\
\hline 1145 & .11 & .48 & .017 & & 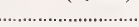 & . & 0.058 \\
\hline 1146 & .20 & 1.51 & .20 & 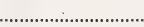 & ………………….... & ․․․․․․․․․…………… & .13 \\
\hline 1150 & .034 & 0.074 & .045 & 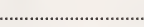 & 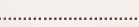 & 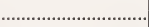 & .014 \\
\hline
\end{tabular}

\section{Nonferrous Alloys (Chip Form)}

These SRM's provide materials of known composition for checking the performance of chemical methods of analysis and in calibration with instrumental methods. The aluminum-, magnesium-, and zincbase alloys are furnished as approximately 0.4 to $1.4 \mathrm{~mm}$ chips prepared by cutting thin wall castings or wrought bar stock. Certificates of Analysis provided with these standards give the composition as determined at NBS, and most give values obtained by industrial and other outside laboratories cooperating in certification of the standards.

Aluminum-Base Alloys

\begin{tabular}{|c|c|c|c|c|c|c|c|c|c|c|c|c|c|c|c|}
\hline \multirow[b]{2}{*}{ SRM } & \multirow[b]{2}{*}{ Type } & \multicolumn{14}{|c|}{ Chemical Composition (Nominal Weight Percent) } \\
\hline & & $\begin{array}{c}\text { Wt/ } \\
\text { Unit } \\
\text { (grams) }\end{array}$ & $\mathrm{Mn}$ & $\mathrm{Si}$ & $\mathrm{Cu}$ & $\mathrm{Ni}$ & $\mathrm{Cr}$ & V & $\mathrm{Ti}$ & Sn & $\mathrm{Ga}$ & $\mathrm{Fe}$ & $\mathrm{Pb}$ & $\mathrm{Mg}$ & $\mathrm{Zn}$ \\
\hline $85 b$ & 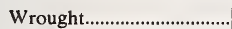 & 75 & 0.61 & 0.18 & 3.99 & 0.084 & 0.211 & 0.006 & 0.022 & & 0.019 & 0.24 & 0.021 & 1.49 & 0.030 \\
\hline $87 a$ & 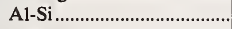 & 75 & .26 & 6.24 & 0.30 & .57 & .11 & $<.01$ & .18 & 0.05 & .02 & .61 & .10 & 0.37 & .16 \\
\hline
\end{tabular}

\section{Copper-Base Alloys}

SRM's $871,872,874,875,879$, and 880 are fine granules produced by a water atomization technique for use primarily in checking chemical methods of analysis. The homogeneity of these materials is exceptionally high, and for certain alloys such as SRM 872, Phosphor Bronze (CDA 544) it is the only form that can readily be prepared to exhibit acceptable homogeneity. For many alloys (for which homogeneity requirements can be met), both granules (or chips) for chemical analysis and solids for optical emission and $\mathrm{x}$-ray fluorescence methods, are desirable. 


\begin{tabular}{|c|c|c|c|c|c|c|c|}
\hline \multirow[b]{2}{*}{ SRM } & \multirow[b]{2}{*}{ Type } & \multicolumn{6}{|c|}{ Chemical Composition (Nominal Weight Percent) } \\
\hline & & $\begin{array}{c}\text { Wt/ } \\
\text { Unit } \\
\text { (grams) }\end{array}$ & $\mathrm{Cu}$ & $\mathrm{Ni}$ & $\mathrm{Fe}$ & $\mathrm{Zn}$ & $\mathrm{Pb}$ \\
\hline $37 \mathrm{e}$ & Brass, Sheet ......... & 150 & 69.61 & 0.53 & 0.004 & 27.85 & 1.00 \\
\hline 158a & Bronze, Silicon ................ & 150 & 90.93 & .001 & 1.23 & 2.08 & 0.097 \\
\hline 184 & Bronze, Leaded Tin & 150 & 88.96 & .50 & .005 & 2.69 & 1.44 \\
\hline 871 & Bronze, Phosphor (CDA 521) & 100 & 91.68 & ............. & $<.001$ & 0.025 & .010 \\
\hline 872 & Bronze, Phosphor (CDA 544) & 100 & 87.36 & & .003 & 4.0 & 4.13 \\
\hline 874 & 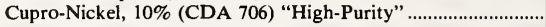 & 100 & 88.49 & 10.18 & 1.22 & 0.002 & $<0.0005$ \\
\hline 875 & Cupro-Nicke1, 10\% (CDA 706) "Doped" & 100 & 87.83 & 10.42 & 1.45 & .11 & .0092 \\
\hline 879 & Nickel Silver (CDA 762) & 100 & 57.75 & 12.11 & 0.0020 & 30.04 & .002 \\
\hline 880 & 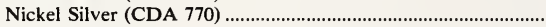 & 100 & 54.51 & 18.13 & .004 & 27.3 & .002 \\
\hline
\end{tabular}

\begin{tabular}{|c|c|c|c|c|c|c|c|c|}
\hline SRM & $\mathrm{Mn}$ & $\mathrm{Sb}$ & Sn & $\mathbf{P}$ & $\mathrm{Si}$ & Al & $\mathrm{Cd}$ & $\mathrm{Se}$ \\
\hline \multirow{3}{*}{$\begin{array}{l}37 \mathrm{e} \\
158 \mathrm{a} \\
184 \\
871\end{array}$} & & 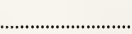 & 1.00 & & & & & \\
\hline & 1.11 & 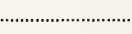 & 0.96 & \multirow{5}{*}{$\begin{array}{l}0.026 \\
.009 \\
.082 \\
.26 \\
.002 \\
.0020\end{array}$} & 3.03 & 0.46 & 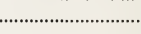 & 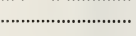 \\
\hline & & & & & & & & . \\
\hline \multirow{5}{*}{$\begin{array}{l}872 \\
874 \\
875 \\
879 \\
880\end{array}$} & & 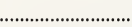 & ............... & & 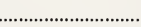 & 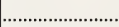 & ……………....... & 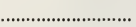 \\
\hline & \multirow{4}{*}{$\begin{array}{l}0.0020 \\
<.0007 \\
<.001 \\
<.001\end{array}$} & $<0.001$ & 0.007 & & $(0.0006)$ & ………………….... & $<0.0002$ & 0.00015 \\
\hline & & $<.001$ & .009 & & $(.0008)$ & $\ldots$ & .0022 & .0004 \\
\hline & & & & ....... & & & & 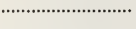 \\
\hline & & & & & 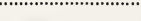 & ................. & .............. & 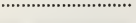 \\
\hline
\end{tabular}

\begin{tabular}{|c|c|c|c|c|c|c|c|c|}
\hline SRM & $\mathrm{Bi}$ & $\mathrm{O}$ & C & $\mathrm{H}$ & $\mathbf{s}$ & As & $\mathrm{Mg}$ & $\mathrm{Ti}$ \\
\hline \multicolumn{9}{|l|}{$37 e$} \\
\hline $158 \mathrm{a}$ & 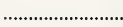 & . & & .. & ...................... & 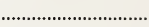 & ……............... & 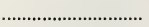 \\
\hline 184 & ․․․․․…………..... & ..... & ......................... & |.................... & ……………....... & .................... & ․․․…………....... & 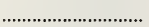 \\
\hline 871 & ..................... & ............ & ……..................... & $\ldots \ldots \ldots \ldots . . .$. & ……..................... & 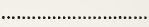 & ………............... & 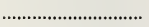 \\
\hline 872 & & & & & & & & \\
\hline 874 & $<0.0002$ & $(0.06)$ & $(0.0028)$ & $(0.0016)$ & $(0.0011)$ & $(<0.0006)$ & $(0.0002)$ & $(0.0001)$ \\
\hline 875 & .003 & (.14) & $(.0035)$ & $(.004)$ & $(.0011)$ & $(.0010)$ & $(.0010)$ & $(<.0002)$ \\
\hline 880 & . & 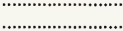 & (1) & 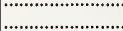 & 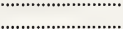 & - & (2) & 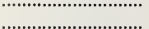 \\
\hline 000 & , & . & . & (1) & & & & . \\
\hline
\end{tabular}

\section{Copper "Benchmark" Standards}

The copper "benchmark" standards were prepared in a cooperative Industry-ASTM-NBS program and were designed primarily for use in calibration with optical emission methods of analysis. They should also serve in the development of other new or improved trace methods of analysis. Twelve different compositions are to be issued as 25 SRM's. $\mathrm{Cu}$ " 0 " and $\mathrm{Cu}$ XI will be issued in chip form only. Cu IV is available only in rod form $6.60 \mathrm{~mm}$ in diameter and $103 \mathrm{~mm}$ long, but is to be issued later as a wateratomized powder. $\mathrm{Cu}$ I, II, III, V, VI, and VII are available both as chips and as rods $6.35 \mathrm{~mm}$ in diameter, $103 \mathrm{~mm}$ long. Cu VIII, IX, and X will be issued as chill-cast and unidirectionally solidfied blocks 32 $\mathrm{mm}$ square and $19 \mathrm{~mm}$ thick. Cu VIII-Cu X are phosphorized copper containing a nominal concentration range from about 10 to $500 \mathrm{ppm}$ for the same 20 trace elements contained in the other copper "benchmark" SRM's, plus 5 to 8 additional elements. These SRM's are applicable for x-ray fluorescence methods of analysis and, because of deliberate additions of gold and silver (in ratios of 1 to 4), for calibration of fire assay equipment. 


\begin{tabular}{|c|c|c|c|c|c|c|c|c|c|c|c|}
\hline \multirow[b]{2}{*}{ SRM } & \multirow[b]{2}{*}{ Type } & \multicolumn{10}{|c|}{ Chemical Composition (Nominal Parts Per Million By Weight) } \\
\hline & & $\begin{array}{c}\text { Wt/ } \\
\text { Unit } \\
\text { (grams) }\end{array}$ & $\mathrm{Sb}$ & As & $\mathrm{Bi}$ & $\mathrm{Cr}$ & Co & $\mathrm{Fe}$ & $\mathrm{Pb}$ & $\mathrm{Mn}$ & $\begin{array}{c}\mathrm{Cu}(\mathrm{Wt} \\
\%)\end{array}$ \\
\hline 393 & Unalloyed-Cu "0" IN PREP.... & & & & & & & & & & \\
\hline 394 & 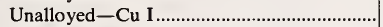 & 50 & 4.5 & 2.6 & 0.35 & 2.0 & 0.5 & 147 & 26.5 & 3.7 & 99.908 \\
\hline 395 & Unalloyed-Cu II ........................................ & 50 & 8.0 & 1.6 & .50 & 6.0 & .3 & 96 & 3.25 & 5.3 & 99.944 \\
\hline 396 & Unalloyed-Cu III............................................. & 50 & $<1$ & $<.2$ & .07 & 4.3 & .4 & 143 & 0.41 & 7.5 & 99.955 \\
\hline 398 & 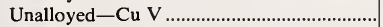 & 50 & 7.5 & 25 & 2.0 & $(0.3)$ & 2.8 & 11.4 & 9.9 & $(0.3)$ & 99.98 \\
\hline 399 & Unalloyed-Cu VI & 50 & 30 & 47 & 10.5 & $(.5)$ & 0.5 & 20.0 & 114 & $(.3)$ & 99.79 \\
\hline 400 & 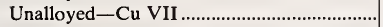 & 50 & 102 & 140 & 24.5 & (.5) & .6 & 41 & 128 & (.2) & 99.70 \\
\hline 454 & Unalloyed-Cu XI.... & & 24 & 46 & 19 & & (4) & (50) & 66 & & 99.84 \\
\hline
\end{tabular}

\begin{tabular}{|c|c|c|c|c|c|c|c|c|c|c|c|c|c|}
\hline SRM & $\mathrm{Ni}$ & $\mathrm{Se}$ & $\mathrm{Ag}$ & $S$ & $\mathrm{Te}$ & Sn & $\mathrm{Zn}$ & Al & $\mathrm{Cd}$ & $\mathrm{Au}$ & $\mathrm{Mg}$ & $\mathrm{O}$ & $\mathrm{Si}$ \\
\hline \multicolumn{14}{|l|}{393} \\
\hline 394 & 11.7 & 2.1 & 50.5 & 15 & 0.57 & 70 & 405 & $(<2)$ & $(0.5)$ & $(0.07)$ & $(<1)$ & (230) & $(<2)$ \\
\hline 395 & 5.4 & 0.60 & 12.2 & 13 & .32 & 1.5 & 12.2 & $(<2)$ & (.4) & (.13) & $(<1)$ & (435) & $(<2)$ \\
\hline 396 & 4.2 & .50 & 3.30 & 9.5 & $<.1$ & 0.8 & 5.0 & $(<2)$ & (.6) & $(<.05)$ & $(<1)$ & (270) & $(<2)$ \\
\hline 398 & 7.0 & 14 & 20.1 & (11) & 11 & 4.8 & 24 & $(<2)$ & (22) & (.1) & $(<1)$ & (30) & $(<2)$ \\
\hline 399 & 506 & $(\sim 95)$ & 116.8 & (10) & $(\sim 50)$ & $(\sim 90)$ & 45 & $(<2)$ & $(<1)$ & (4) & $(<1)$ & $(950)$ & $(<2)$ \\
\hline 400 & 603 & $(\sim 250)$ & 181 & (9) & $(\sim 155)$ & $(\sim 200)$ & 114 & $(<2)$ & $(<1)$ & (10) & $(<1)$ & (1025) & $(<2)$ \\
\hline 454 & (150) & 400 & 286 & & & 2.2 & 7 & & & 7.5 & & & \\
\hline
\end{tabular}

Lead-Base Alloys

\begin{tabular}{|c|c|c|c|c|c|c|c|c|c|c|c|}
\hline \multirow[b]{2}{*}{ SRM } & \multirow[b]{2}{*}{ Type } & \multicolumn{10}{|c|}{ Chemical Composition (Nominal Weight Percent) } \\
\hline & & $\begin{array}{l}\text { Wt/ } \\
\text { Unit } \\
\text { (gram) }\end{array}$ & $\begin{array}{l}\text { (Other } \\
\text { Forms) }\end{array}$ & $\mathrm{Cu}$ & $\mathrm{Ni}$ & As & Sn & $\mathrm{Sb}$ & $\mathrm{Bi}$ & $\mathrm{Ag}$ & $\mathrm{Fe}$ \\
\hline $53 \mathrm{e}$ & Bearing Metal $(84 \mathrm{~Pb}-10 \mathrm{Sb}-6 \mathrm{Sn}) \ldots .$. & 150 & 1132 & 0.054 & 0.003 & 0.057 & 5.84 & 10.26 & 0.052 & & $<0.001$ \\
\hline $127 \mathrm{~b}$ & Solder $(40 \mathrm{Sn}-60 \mathrm{~Pb})$ & 150 & 1131 & .011 & .012 & .01 & 39.3 & 0.43 & .06 & 0.001 & \\
\hline
\end{tabular}

\section{Magnesium-Base Alloys}

\begin{tabular}{|c|c|c|c|c|c|c|c|c|c|c|}
\hline \multirow[b]{2}{*}{ SRM } & \multirow[b]{2}{*}{ Type } & \multicolumn{9}{|c|}{ Chemical Composition (Normal Weight Percent) } \\
\hline & & $\begin{array}{l}\text { Wt/Unit } \\
\text { (grams) }\end{array}$ & Mn & $\mathrm{Si}$ & $\mathrm{Cu}$ & $\mathrm{Ni}$ & A1 & $\mathrm{Pb}$ & $\mathrm{Fe}$ & $\mathrm{Zn}$ \\
\hline 171 & Alloy ................ & 100 & 0.45 & 0.0118 & 0.0112 & 0.0009 & 2.98 & 0.0033 & 0.0018 & 1.05 \\
\hline
\end{tabular}

Nickel-Base Alloys

\begin{tabular}{|c|c|c|c|c|c|c|c|c|c|c|}
\hline \multirow[b]{2}{*}{ SRM } & \multirow[b]{2}{*}{ Type } & \multicolumn{9}{|c|}{ Chemical Composition (Nominal Weight Percent) } \\
\hline & & $\begin{array}{c}\text { Wt/ } \\
\text { Unit } \\
\text { (grams) }\end{array}$ & $\mathrm{C}$ & Mn & $\mathbf{P}$ & s & $\mathrm{Si}$ & $\mathrm{Cu}$ & $\mathrm{Ni}$ & $\mathrm{Cr}$ \\
\hline 349 & Ni57-Cr20 & 150 & 0.08 & 0.43 & 0.002 & & 0.29 & 0.006 & 57.15 & 19.50 \\
\hline 882 & Ni66-Cu31-A13 ....................................... & 150 & .006 & .0007 & ..................... & 0.0014 & .006 & 31.02 & 65.25 & \\
\hline
\end{tabular}




\begin{tabular}{|c|c|c|c|c|c|c|c|c|c|c|c|}
\hline SRM & v & Mo & W & Co & $\mathrm{Ti}$ & $\mathrm{Al}$ & B & $\mathrm{Fe}$ & $\mathrm{Nb}$ & $\mathrm{Ta}$ & $\mathrm{Zr}$ \\
\hline \multirow{2}{*}{$\begin{array}{l}349 \\
882\end{array}$} & 0.081 & 4.04 & $<0.01$ & 13.95 & \multirow{2}{*}{$\begin{array}{l}3.05 \\
0.57\end{array}$} & \multirow{2}{*}{$\begin{array}{l}1.23 \\
2.85\end{array}$} & 0.0046 & \multirow{2}{*}{$\begin{array}{c}0.13 \\
.009\end{array}$} & $<0.01$ & $<0.01$ & 0.081 \\
\hline & . & & & & & & & & ......... & …… & ........ \\
\hline
\end{tabular}

\section{Trace Elements in Nickel-Base Superalloy}

The Gas Turbine Panel of the ASTM-ASME-MPG Joint Committee, through recommendation of its Task Force on Trace Elements in Superalloys, prepared a set of three "Tracealloy" materials, which have the same common matrix. These were given to NBS for "definitive analysis" of: $\mathrm{Pb}, \mathrm{Bi}, \mathrm{Se}, \mathrm{Te}$, and $\mathrm{Tl}$; trace elements that vary over the concentration range of interest. These SRM's 897,898 , and 899 are in the form of fine articles.

\begin{tabular}{|c|c|c|c|c|c|c|}
\hline \multirow{2}{*}{ SRM } & \multirow{2}{*}{ Type } & \multicolumn{5}{|c|}{ Nominal Trace Composition (Parts Per Million by Weight) } \\
\hline & & $\mathrm{Pb}$ & $\mathrm{Bi}$ & $\mathrm{Se}$ & $\mathrm{Te}$ & $\mathrm{T} 1$ \\
\hline 897 & "Tracealloy" A & 14.7 & $(0.53)$ & 9.1 & 1.05 & 0.51 \\
\hline 898 & "Tracealloy" В & 3.1 & (1.1) & 2.00 & 0.54 & 2.75 \\
\hline 899 & "Tracealloy" C & 4.9 & $(0.26)$ & 9.5 & 5.9 & 0.252 \\
\hline
\end{tabular}

\begin{tabular}{|c|c|c|c|c|c|c|c|c|c|c|c|c|}
\hline & \multicolumn{12}{|c|}{ Approximate Base Composition (Weight Percent) } \\
\hline & $\mathrm{C}$ & $\mathrm{Cr}$ & Co & $\mathrm{Ni}$ & W & $\mathrm{Nb}$ & Al & $\mathrm{Ti}$ & B & $\mathrm{Zr}$ & $\mathrm{Ta}$ & Hf \\
\hline 897 & $(0.12)$ & $(12.0)$ & $(8.5)$ & (Bal) & $(1.75)$ & $(0.9)$ & $(2.0)$ & $(2.0)$ & $(0.010)$ & $(0.10)$ & $(1.75)$ & $(1.2)$ \\
\hline 898 & $(0.12)$ & $(12.0)$ & $(8.5)$ & (Bal) & $(1.75)$ & $(0.9)$ & (2.0) & $(2.0)$ & $(0.010)$ & $(0.10)$ & (1.75) & $(1.2)$ \\
\hline 899 & $(0.12)$ & $(12.0)$ & $(8.5)$ & (Bal) & (1.75) & $(0.9)$ & $(2.0)$ & $(2.0)$ & $(0.010)$ & $(0.10)$ & (1.75) & (1.2) \\
\hline
\end{tabular}

\section{Nickel Oxide}

The nickel oxide SRM's are available primarily for application in the electronics industry to the analysis of cathode grade nickel. The "Standard Method for Spectrochemical Analysis of Thermionic Nickel Alloys by the Powder-DC Arc Technique," ASTM Designation E129, is based on calibration with these standards. The values given are for the percentage of the element in nickel oxide. Values in parentheses are not certified, but are given for information only.

\begin{tabular}{|c|c|c|c|c|c|c|c|c|c|c|c|}
\hline \multirow[b]{2}{*}{ SRM } & \multirow[b]{2}{*}{ Type } & \multicolumn{10}{|c|}{ Chemical Composition (nominal Weight Percent) } \\
\hline & & $\begin{array}{c}\text { Wt/ } \\
\text { Unit } \\
\text { (grams) }\end{array}$ & $\mathrm{Mn}$ & $\mathrm{Si}$ & $\mathrm{Cu}$ & $\mathrm{Cr}$ & Co & $\mathrm{Ti}$ & Al & $\mathrm{Fe}$ & $\mathrm{Mg}$ \\
\hline 671 & Oxide 1 & 25 & 0.13 & 0.047 & 0.20 & 0.025 & 0.31 & 0.024 & 0.009 & 0.39 & 0.030 \\
\hline 672 & Oxide 2 & 25 & .095 & .11 & .018 & .003 & .55 & .009 & .004 & .079 & .020 \\
\hline 673 & Oxide 3 . & 25 & .0037 & .006 & .002 & .0003 & .016 & .003 & .001 & .029 & .003 \\
\hline
\end{tabular}




\begin{tabular}{|c|c|c|c|c|c|c|c|c|c|c|c|c|}
\hline \multirow{2}{*}{ SRM } & \multicolumn{12}{|c|}{ Nominal Trace Composition (Parts Per Million by Weight) } \\
\hline & $\mathrm{Pb}$ & $\mathrm{Se}$ & $\mathrm{Bi}$ & As & Sn & $\mathrm{Sb}$ & $\mathrm{Cd}$ & $\mathrm{Ga}$ & $\mathrm{Ag}$ & Te & $\mathrm{T} 1$ & $\mathrm{Zn}$ \\
\hline 671 & 16 & 2.0 & 0.07 & (59) & (2.7) & $(0.4)$ & $(0.7)$ & $(0.8)$ & $(0.5)$ & $(<0.2)$ & $(<0.1)$ & $(160)$ \\
\hline 672 & 38 & 0.40 & .3 & (74) & (4) & $(.5)$ & (1.7) & (.4) & (.3) & $(<.2)$ & $(<.1)$ & (140) \\
\hline 673 & 3.5 & .2 & .06 & (0.4) & $(<0.5)$ & $(<.05)$ & $(0.05)$ & $(<.1)$ & $(<.1)$ & (.4) & $(<.1)$ & (1.7) \\
\hline
\end{tabular}

Selenium

\begin{tabular}{|c|c|c|c|c|c|c|c|c|c|c|c|c|c|c|c|c|c|}
\hline \multirow[b]{2}{*}{ SRM } & \multirow{2}{*}{\multicolumn{6}{|c|}{ Type }} & \multicolumn{11}{|c|}{ Chemical Composition (Nominal Parts Per Million) } \\
\hline & & & & & & & $\underset{\text { (grams) }}{\mathrm{Wt} /}$ & Mn & $\mathbf{S}$ & $\mathrm{Cu}$ & $\mathrm{Ni}$ & $\mathrm{Cr}$ & $\mathbf{V}$ & Mo & Co & As & Sn \\
\hline 726 & Seleniun & Interm & diate $\mathbf{P}$ & ity ......... & $\ldots \ldots \ldots$ & ........ & 450 & $<0.3$ & $12 \pm 3$ & $<1$ & $<0.5$ & $<1$ & N.D. & $<0.3$ & N.D. & $<2$ & $<1$ \\
\hline SRM & Al & B & $\mathrm{Pb}$ & $\mathbf{B i}$ & $\mathrm{Ag}$ & $\mathrm{Ca}$ & $\mathbf{M g}$ & & $\mathrm{Te}$ & $\mathrm{Fe}$ & $\mathrm{Cl}$ & & $\mathrm{Ti}$ & $\mathrm{Be}$ & $\mathrm{Cd}$ & & In \\
\hline 726 & $<1$ & $<1$ & $<1$ & N.D. & $<1$ & $<1$ & $<$ & & $0.3 \pm 0.1$ & 1 & & 0.5 & $<0.5$ & N.D. & N.I & & N.D. \\
\hline
\end{tabular}

N.D. $=$ Not detected at limits of detection of $<0.5 \mathrm{ppm}$.

Tin-Base Alloys

\begin{tabular}{|c|c|c|c|c|c|c|c|c|c|c|c|}
\hline \multirow[b]{2}{*}{ SRM } & \multirow[b]{2}{*}{ Type } & \multicolumn{10}{|c|}{ Chemical Composition (Nominal Weight Percent) } \\
\hline & & $\underset{\text { Unit }}{\text { Ut/ }}$ & $\mathrm{Pb}$ & Sn & $\mathrm{Sb}$ & $\mathbf{B i}$ & $\mathrm{Cu}$ & $\mathrm{Fe}$ & As & $\mathrm{Ag}$ & $\mathrm{Ni}$ \\
\hline $54 d$ & Bearing Metal & 170 & 0.62 & 88.57 & 7.04 & 0.044 & 3.62 & 0.027 & 0.088 & 0.0032 & 0.0027 \\
\hline
\end{tabular}

Titanium-Base Alloys

\begin{tabular}{|c|c|c|c|c|c|c|c|c|c|c|c|c|}
\hline \multirow[b]{2}{*}{ SRM } & \multirow[b]{2}{*}{ Type } & \multicolumn{11}{|c|}{ Chemical Composition (Nominal Weight Percent) } \\
\hline & & $\begin{array}{c}\text { Wt/ } \\
\text { Unit } \\
\text { (grams) }\end{array}$ & $\mathrm{C}$ & $\mathrm{Mn}$ & $\mathrm{Si}$ & $\mathrm{Cu}$ & V & Mo & Sn & Al & $\mathrm{Fe}$ & $\mathbf{N}$ \\
\hline $173 b$ & 6Al-4V (IN PREP) & & & & & & & & & & & \\
\hline 174 & 4Al-4Mn & 100 & ............. & 4.57 & 0.015 & & & & & 4.27 & 0.175 & 0.012 \\
\hline 176 & 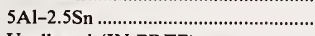 & 100 & .015 & 0.0008 & & .003 & & .0003 & 2.47 & 5.16 & .070 & .010 \\
\hline 650 & Unalloyed (IN PREP) & & & & & & & & & & & \\
\hline 651 & 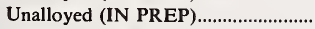 & & & & & & & & & & & \\
\hline 652 & 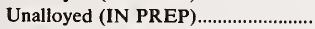 & ............ & & & & & & & & & & (..................... \\
\hline
\end{tabular}


Zinc-Base

\begin{tabular}{|c|c|c|c|c|c|c|c|c|c|c|c|c|c|}
\hline \multirow{2}{*}{ SRM } & \multirow{2}{*}{ Type } & \multirow{2}{*}{$\begin{array}{c}\text { Wt/ } \\
\text { Unit } \\
\text { (grams) }\end{array}$} & \multicolumn{11}{|c|}{ Chemical Composition (Nominal Weight Percent) } \\
\hline & & & Mn & $\mathrm{Cu}$ & $\mathrm{Ni}$ & Sn & Al & $\mathrm{Cd}$ & $\mathrm{Fe}$ & $\mathrm{Pb}$ & $\mathrm{Ag}$ & $\mathrm{Mg}$ & $\mathrm{Ti}$ \\
\hline $94 \mathrm{c}$ & $\begin{array}{l}\text { Die Casting } \\
\text { Alloy. }\end{array}$ & 150 & 0.014 & 1.01 & 0.006 & 0.006 & 4.13 & 0.002 & 0.018 & 0.006 & & 0.042 & …... \\
\hline 728 & 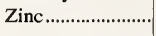 & 450 & ............. & 0.00057 & (n).............. & $(.000002)$ & ............ & .00012 & .00027 & .00111 & 0.00011 & & ....... \\
\hline
\end{tabular}

Zirconium-Base

\begin{tabular}{|c|c|c|c|c|c|c|c|c|c|c|c|c|c|}
\hline \multirow[b]{2}{*}{ SRM } & \multirow[b]{2}{*}{ Type } & \multicolumn{12}{|c|}{ Chemical Composition (Nominal Parts Per Million) } \\
\hline & & $\begin{array}{c}\text { Wt/ } \\
\text { Unit } \\
\text { (grams) }\end{array}$ & $\mathrm{C}$ & $\mathrm{Mn}$ & $\mathrm{Si}$ & $\mathrm{Cu}$ & $\mathrm{Ni}$ & $\mathrm{Cr}$ & $\mathrm{Ti}$ & $\begin{array}{c}\mathrm{Sn} \\
(\mathrm{Wt} \%)\end{array}$ & $\mathrm{Fe}$ & $\mathbf{N}$ & $\mathbf{U}$ \\
\hline $360 \mathrm{a}$ & Zircaloy-2 ............................................... & 100 & 136 & 3 & 51 & 140 & 554 & 1060 & 27 & 1.42 & 1441 & 43 & 0.15 \\
\hline
\end{tabular}

\section{Nonferrous Alloys (Solid Form)}

These SRM's are designed to fill the basic needs of the nonferrous primary and secondary metals industries for analytical control, primarily with optical emission and x-ray spectroscopic methods. Both nominal chemical composition and analytical range SRM's have been prepared for many of the commercially important nonferrous alloy systems.

\section{Aluminum "Benchmark" Standards}

Aluminum "benchmark" standards are being prepared in a cooperative Industry-ASTM-NBS program. Five SRM's in disk form, are intended for use primarily in optical emission and x-ray spectrometric methods of analysis. Selection was made to include (annealed) aluminum alloys, 6011 (Modified) and 7075/ 7078; two large tonnage casting alloys 356 and 380; and a high purity aluminum. These materials will also be available in the form of chips, for checking chemical methods of analysis (See page 31).

\begin{tabular}{|c|c|c|c|c|c|c|c|c|c|c|c|c|c|c|}
\hline \multirow{2}{*}{ SRM } & \multirow{2}{*}{ Type } & \multirow{2}{*}{ Size } & \multicolumn{12}{|c|}{ Chemical Composition (Nominal Weight Percent) } \\
\hline & & & $\mathrm{Si}$ & $\mathrm{Fe}$ & $\mathrm{Cu}$ & Mn & $\mathrm{Cr}$ & $\mathrm{Ni}$ & $\mathrm{Zn}$ & $\mathrm{Mg}$ & $\mathrm{Be}$ & $\mathrm{Ti}$ & $\mathrm{Pb}$ & Sn \\
\hline 1255 & Casting (356)....... & $\begin{array}{l}64 \mathrm{~mm} \text { dia } X \\
19 \mathrm{~mm} \text { thick }\end{array}$ & 7.17 & 0.15 & 0.12 & 0.055 & 0.013 & 0.014 & 0.083 & 0.35 & & 0.15 & 0.015 & 0.018 \\
\hline 1256 & $\begin{array}{l}\text { Casting (A380) } \\
\text { (IN PREP). }\end{array}$ & $\begin{array}{l}64 \mathrm{~mm} \text { dia } \mathrm{X} \\
19 \mathrm{~mm} \text { thick }\end{array}$ & 9.18 & .94 & 3.51 & .35 & .055 & .37 & .96 & .063 & & .077 & .10 & .10 \\
\hline 1257 & Pure Aluminum . & (IN PREP) & & & & & & & & & & & & \\
\hline 1258 & Alloy $6011 \ldots . . . . . .$. & $\begin{array}{l}35 \mathrm{~mm} \text { dia } \mathrm{X} \\
19 \mathrm{~mm} \text { thick }\end{array}$ & .78 & .079 & .84 & .48 & .0011 & .0006 & 1.03 & .98 & $<0.0001$ & & & ........... \\
\hline 1259 & Alloy $7075 \ldots \ldots \ldots$ & $\begin{array}{l}35 \mathrm{~mm} \text { dia } X \\
19 \mathrm{~mm} \text { thick }\end{array}$ & .18 & .205 & 1.60 & .079 & .173 & .063 & 5.44 & 2.48 & .0025 & & & \\
\hline
\end{tabular}


A number of copper-base alloy SRM's were prepared to provide for analytical control by rapid instrumental methods in the copper industry. These SRM's are for calibration of optical emission and x-ray spectroscopic equipment. Eight groups were prepared in two forms: chill-cast (with "C" prefix) for the producer (blocks, $31 \mathrm{~mm}$ square, $19 \mathrm{~mm}$ thick), and wrought for the consumer (disks, $31 \mathrm{~mm}$ in diameter and 19 $\mathrm{mm}$ thick). Both forms have nearly identical chemical compositions. Consequently, when the supply of one form is exhausted, the other is the recommended replacement. For each of the eight principal copper-base alloys, three SRM's were prepared to comprise a "nominal-composition", and both a low- and high-composition standard. To make the cartridge-brass SRM's more widely applicable, a number of trace elements were purposely added and certified. The beryllium copper SRM's are representative of the nominal chemical composition of three Copper and Brass Research Association (CABRA) alloy designations. Values in parentheses are not certified, but are given for information only.

\begin{tabular}{|c|c|c|c|c|c|c|c|c|c|c|c|}
\hline \multirow{2}{*}{ SRM } & & \multirow{2}{*}{ Type } & \multicolumn{9}{|c|}{ Chemical Composition (Nominal Weight Percent) } \\
\hline & & & $\mathrm{Cu}$ & $\mathrm{Zn}$ & $\mathrm{Pb}$ & $\mathrm{Fe}$ & Sn & $\mathrm{Ni}$ & A1 & $\mathrm{Sb}$ & As \\
\hline & C1101 & 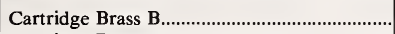 & 69.50 & 30.30 & 0.05 & 0.037 & 0.016 & 0.013 & 0.0006 & 0.012 & 0.009 \\
\hline 1102 & ................... & 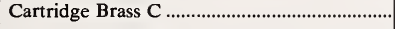 & 72.85 & 27.10 & .020 & .011 & .006 & .005 & .0007 & .005 & .004 \\
\hline 1103 & & Free-Cutting Brass A & 59.23 & 35.7 & 3.73 & .26 & .88 & .16 & & & \\
\hline 1104 & C1104 & Free-Cutting Brass B & 61.33 & 35.3 & 2.77 & .088 & .43 & .070 & & & .......... \\
\hline ................ & C1105 & 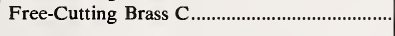 & 63.7 & 34.0 & 2.0 & .044 & .21 & .043 & & & ................... \\
\hline 1106 & C1106 & 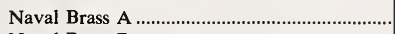 & 59.08 & 40.08 & 0.032 & .004 & .74 & .025 & & & \\
\hline 1107 & C1107 & 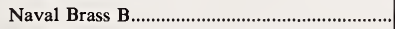 & 61.21 & 37.34 & .18 & .037 & 1.04 & .098 & & & \\
\hline 1108 & C1108 & Naval Brass C & 64.95 & 34.42 & .063 & .050 & 0.39 & .033 & & & 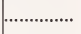 \\
\hline 1109 & C1109 & 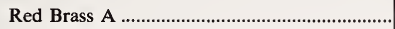 & 82.2 & 17.4 & .075 & .053 & .10 & .10 & & & \\
\hline 1110 & C1110 & 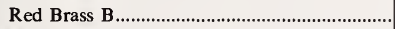 & 84.59 & 15.20 & .033 & .033 & .051 & .053 & & & \\
\hline 1111 & C1111 & Red Brass C & 87.14 & 12.81 & .013 & .010 & .019 & .022 & & & \\
\hline 1112 & $\mathrm{C} 1112$ & Gilding Metal A & 93.38 & 6.30 & .057 & .070 & .12 & .100 & & & \\
\hline 1113 & $\mathrm{C} 1113$ & Gilding Metal B & 95.03 & 4.80 & .026 & .043 & .064 & .057 & & & \\
\hline 1114 & C1114 & 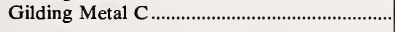 & 96.45 & 3.47 & .012 & .017 & .027 & .021 & & & ................. \\
\hline 1115 & C1115 & 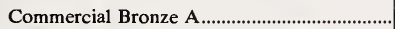 & 87.96 & 11.73 & .013 & .13 & .10 & .074 & & & \\
\hline 1116 & $\mathrm{C} 1116$ & 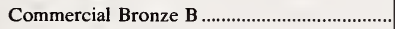 & 90.37 & 9.44 & .042 & .046 & .044 & .048 & & & ........ \\
\hline 1117 & C1117 & 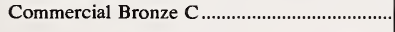 & 93.01 & 6.87 & .069 & .014 & .021 & .020 & & & ............ \\
\hline 1118 & $\mathrm{C} 1118$ & Aluminum Brass A & 75.1 & 21.9 & .025 & .065 & & & 2.80 & .010 & .007 \\
\hline 1119 & C1119 & 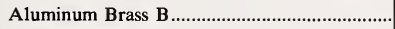 & 77.1 & 20.5 & .050 & .030 & & & 2.14 & .050 & .040 \\
\hline 1120 & C1120 & 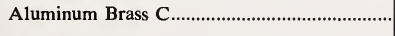 & 80.1 & 18.1 & .105 & .015 & & .............. & 1.46 & .100 & .090 \\
\hline .................... & C1121 & 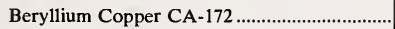 & 97.49 & $(0.01)$ & $(.002)$ & .085 & .01 & .012 & 0.07 & & \\
\hline 1122 & $\mathrm{C} 1122$ & 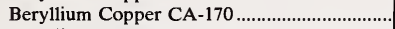 & 97.45 & $(.01)$ & $(.003)$ & .16 & $(.01)$ & $(.01)$ & .17 & & \\
\hline 1123 & C1123 & Beryllium Copper CA-175 ............................. & 97.10 & $(.01)$ & $(.001)$ & .04 & $(.01)$ & $(.01)$ & .02 & & \\
\hline 1275 & .................. & 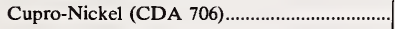 & 88.2 & .085 & .006 & 1.46 & .008 & $9.7 \overline{6}$ & & .0005 & $(\leqslant 0.001)$ \\
\hline 1276 & .................. & 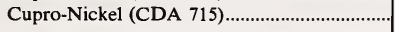 & 67.8 & .038 & .004 & 0.56 & 20.3 & B0.5 & 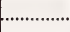 & .0004 & $(\leqslant .001)$ \\
\hline
\end{tabular}




\begin{tabular}{|c|c|c|c|c|c|c|c|c|c|c|c|c|c|c|c|c|}
\hline SRM & & $\mathrm{Be}$ & $\mathrm{Bi}$ & $\mathrm{Cd}$ & Mn & $\mathbf{P}$ & $\mathrm{Si}$ & $\mathrm{Ag}$ & $\mathrm{Te}$ & Co & $\mathrm{Cr}$ & $\mathrm{Se}$ & $\mathrm{Mg}$ & B & $\mathrm{s}$ & $\mathrm{Ti}$ \\
\hline & C1101 & 0.00055 & 0.0004 & 0.0055 & 0.0055 & \multirow{5}{*}{$\begin{array}{r}0.0020 \\
.0048 \\
\\
.003 \\
.005 \\
.003\end{array}$} & $(0.005)$ & \multirow{3}{*}{$\begin{array}{l}0.003 \\
.0010\end{array}$} & \multirow{3}{*}{$\begin{array}{r}0.0015 \\
.0003\end{array}$} & & & & & & & \\
\hline 1102 & ……........ & .00003 & .0005 & .0045 & .0045 & & .002 & & & & & & & & & ........... \\
\hline \multirow{3}{*}{1104} & C1103. & & & & & & & & & & & & & & & \\
\hline & C1104. & & & & & & & & & & & & & & & .................. \\
\hline & C1105 & & & & .................. & & & & & & & & & & & \\
\hline 1106 & C1106 & & & & .005 & & & & & & & & & & & \\
\hline 1107 & C1107. & & & & & & & & & & & & & & & \\
\hline 1108 & $\mathrm{C} 1108$ & ... & …… & .............. & .025 & & & ....... & .......... & ...... & 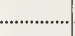 & ...... & & ........... & & 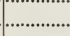 \\
\hline 1109 & C1109 & & & & & .006 & & & & & & & & & & \\
\hline 1110 & C1110 & & & & & & & & & & & & & 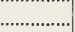 & & 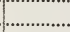 \\
\hline 1111 & C1111 & & & & & & & & & & & & & & & ...................... \\
\hline 1112 & C1112. & & & & & .009 & & & & & & & & & & \\
\hline 1113 & C1113. & & & & & .008 & & & & & & & & & & \\
\hline 1114 & C1114. & & & & ................. & .009 & 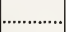 & 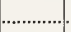 & $\ldots$ & $\ldots$ & . & ........ & ....... & ....... & ....... & …......... \\
\hline 1115 & C1115 & & & & & .005 & & & & & & & & & & \\
\hline 1116 & C1116 & & & & 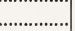 & .008 & & & & & & & & & & 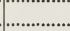 \\
\hline 1117 & C1117 & & & & & .002 & & & & & & & & & & 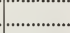 \\
\hline 1118 & C1118 & & & & & .13 & .0021 & & & & & & & & & \\
\hline 1119 & C1119 & & & & 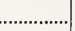 & .070 & .0015 & & & & & & & & & ............ \\
\hline 1120 & $\mathrm{C} 1120$ & .... & .............. & & ................. & .018 & .0011 & & $\ldots$ & .......... & & & & & & $\ldots \ldots . .$. \\
\hline & $\mathrm{C} 1121$ & 1.90 & & & $(.004)$ & $(.005)$ & .11 & $(.005)$ & & 0.295 & $(0.002)$ & & & & & \\
\hline 1122 & C1122 & 1.75 & ............ & . & $(.004)$ & $(.004)$ & .17 & $(.005)$ & 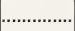 & .220 & $(.002)$ & & & & & .......... \\
\hline 1123 & C1123 & 0.46 & & ............. & $(.002)$ & $(.002)$ & .03 & $(.009)$ & 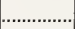 & 2.35 & $(.002)$ & & & & & .......... \\
\hline 1275 & & & $(\leqslant 0.001)$ & .0003 & .42 & .005 & $(.001)$ & $(.004)$ & $(.0002)$ & 0.024 & $(.0002)$ & 0.0004 & 0.003 & $(0.0009)$ & $(0.008)$ & $(0.0002)$ \\
\hline 1276 & & .................... & $(\leqslant .0001)$ & .0002 & 1.01 & .006 & $(.001)$ & $(.004)$ & $(.0002)$ & .045 & $(.0002)$ & .0005 & .12 & $(.0001)$ & $.008)$ & $(.0002)$ \\
\hline
\end{tabular}

\section{Copper "Benchmark" Standards}

The copper "benchmark" standards were prepared in a cooperative Industry-ASTM-NBS program and were designed primarily for use in calibration with optical emission methods of analysis. They should also serve in the development of other new or improved trace methods of analysis. Twelve different compositions are to be issued as 25 SRM's $\mathrm{Cu}$ " $\mathrm{O}$ " and $\mathrm{Cu}$ XI will be issued in chip form only. Cu IV is available only in rod form $6.60 \mathrm{~mm}$ in diameter and $103 \mathrm{~mm}$ long, but is to be issued later as a wateratomized powder. Cu I, II, III, V, VI, VII are available both as chips and as rods $6.35 \mathrm{~mm}$ in diameter, $103 \mathrm{~mm}$ long. $\mathrm{Cu}$ VIII, IX, and X will be issued as chill-cast and unidirectionally solidfied blocks $32 \mathrm{~mm}$ square and $19 \mathrm{~mm}$ thick, $\mathrm{Cu}$ VIII-Cu X are phosphorized copper containing a nominal concentration range from about 10 to $500 \mathrm{ppm}$ for the same 20 trace elements contained in the other copper "benchmark" SRM's, plus 5 to 8 additional elements. These SRM's are applicable for x-ray fluorescence methods of analysis and, because of deliberate additions of gold and silver (in ratios of 1 to 4), for calibration of fire assay equipment. 


\begin{tabular}{|c|c|c|c|c|c|c|c|}
\hline \multirow{2}{*}{ SRM } & \multirow{2}{*}{ Type } & \multicolumn{6}{|c|}{ Chemical Composition (Nominal Parts Per Million) } \\
\hline & & Form & $\mathrm{Cu}(\mathrm{Wt} \%)$ & $\mathrm{Sb}$ & As & $\mathrm{Bi}$ & $\mathrm{Fe}$ \\
\hline 494 & 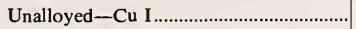 & rod & 99.91 & 4.5 & 2.6 & 0.35 & $(\sim 155)$ \\
\hline 495 & 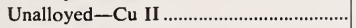 & rod & 99.94 & 8.0 & 1.6 & .50 & $(\sim 100)$ \\
\hline 496 & 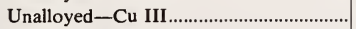 & rod & 99.95 & $<1$ & $<0.2$ & .07 & $(\sim 150)$ \\
\hline 457 & 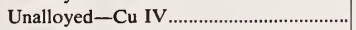 & rod & 99.96 & 0.2 & 0.2 & .2 & 2.0 \\
\hline 498 & Unalloyed-Cu V & rod & 99.98 & 7.4 & 25 & 2.0 & 11 \\
\hline 499 & 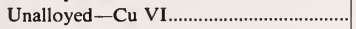 & rod & 99.79 & 30 & 47 & 10.5 & 21 \\
\hline 500 & 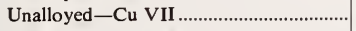 & rod & 99.70 & 100 & 140 & 25 & 42 \\
\hline $\mathrm{C} 1251$ & 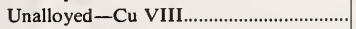 & disk & 99.96 & 12.6 & $(8)$ & (3) & $(10)$ \\
\hline $\mathrm{C} 1252$ & Unalloyed-Cu IX & disk & 99.89 & 42 & 124 & 20 & $(40)$ \\
\hline $\mathrm{C} 1253$ & Unalloyed-Cu X & disk & 99.42 & $(132)$ & 244 & 70 & $(300)$ \\
\hline
\end{tabular}

\begin{tabular}{|c|c|c|c|c|c|c|c|c|}
\hline SRM & $\mathrm{Pb}$ & Mn & $\mathrm{Ni}$ & $\mathrm{Se}$ & $\mathrm{Ag}$ & $\mathrm{Te}$ & Sn & $\mathrm{Zn}$ \\
\hline 494 & 26.5 & 3.7 & 11.7 & 2.1 & 50 & 0.6 & 70 & 400 \\
\hline 495 & 3.2 & 5.3 & 5.4 & 0.6 & 12.2 & .3 & 1.5 & 12 \\
\hline 496 & 0.4 & 7.5 & 4.2 & .5 & 3.3 & $<.1$ & 0.8 & 5.0 \\
\hline 457 & .5 & $<0.1$ & 0.6 & 3.3 & 8.1 & .5 & $<.2$ & $<11$ \\
\hline 498 & 10 & (.3) & 7.0 & 14 & 20.1 & 11 & 5 & 25 \\
\hline 499 & 114 & (.3) & 504 & $(\sim 90)$ & 114 & $(\sim 50)$ & $(\sim 90)$ & 41 \\
\hline 500 & 128 & (.2) & 603 & $(\sim 200)$ & 176 & $(\sim 155)$ & $(\sim 250)$ & 111 \\
\hline $\mathrm{C} 1251$ & 7.5 & (7) & 22 & 8.6 & 81.4 & (12) & (15) & 8.3 \\
\hline $\mathrm{C} 1252$ & 60 & (28) & 128 & 46 & 166.6 & (44) & (124) & 60 \\
\hline $\mathrm{C} 1253$ & 244 & {$[\sim 300]$} & $(500)$ & 140 & 503 & (193) & (489) & 368 \\
\hline
\end{tabular}

\begin{tabular}{|c|c|c|c|c|c|c|c|c|}
\hline SRM & $\mathrm{Al}$ & $\mathrm{Cd}$ & $\mathrm{Cr}$ & Co & $\mathrm{Au}$ & $\mathrm{Mg}$ & $\mathrm{O}$ & $\mathrm{S}$ \\
\hline 494 & $(<2)$ & $(0.5)$ & 2.0 & 0.5 & $(0.07)$ & $(<1)$ & $(230)$ & 15 \\
\hline 495 & $(<2)$ & $(.4)$ & 6.0 & .3 & (.13) & $(<1)$ & (435) & 13 \\
\hline 496 & $(<2)$ & (.6) & 4.3 & .4 & $(<.05)$ & $(<1)$ & $(270)$ & 9 \\
\hline 457 & $(<2)$ & $(<1)$ & $(0.3)$ & $(.2)$ & $(<.05)$ & $(<1)$ & $(360)$ & (4) \\
\hline 498 & $(<2)$ & (22) & $(.3)$ & 2.7 & (.1) & $(<1)$ & (30) & (11) \\
\hline 499 & $(<2)$ & $(<1)$ & (.5) & 0.5 & (4) & $(<1)$ & (950) & (10) \\
\hline 500 & $(<2)$ & $(<1)$ & (.5) & .5 & $(10)$ & $(<1)$ & $(1025)$ & (9) \\
\hline $\mathrm{C} 1251$ & {$[\sim 5]$} & {$[\sim 3]$} & 2.8 & 8.8 & 15.0 & $(10)$ & {$[\sim 120]$} & (22) \\
\hline $\mathrm{C} 1252$ & {$[\sim 40]$} & {$[\sim 15]$} & 7.4 & 90 & 34.9 & (20) & {$[\sim 150]$} & (29) \\
\hline $\mathrm{C} 1253$ & {$[\sim 160]$} & {$[\sim 60]$} & (187) & $(510)$ & 74.4 & (80) & {$[\sim 85]$} & (50) \\
\hline
\end{tabular}

Lead-Base Alloys

\begin{tabular}{|c|c|c|c|c|c|c|c|c|c|c|}
\hline \multirow{2}{*}{$\begin{array}{c}\text { SRM } 31.4 \\
\text { mm D } \times 19 \\
\text { mm thick }\end{array}$} & \multirow[b]{2}{*}{ Type } & \multicolumn{9}{|c|}{ Chemical Composition (Nominal Weight Percent) } \\
\hline & & $\begin{array}{l}\text { Other } \\
\text { Forms }\end{array}$ & $\mathrm{Cu}$ & $\mathrm{Ni}$ & As & Sn & $\mathrm{Sb}$ & $\mathrm{Bi}$ & $\mathrm{Ag}$ & $\mathrm{Fe}$ \\
\hline 1131 & Solder Pb60-Sn40 & $127 \mathrm{~b}$ & 0.011 & 0.012 & 0.01 & 39.3 & 0.43 & 0.06 & 0.01 & \\
\hline 1132 & Bearing Metal & $53 \mathrm{e}$ & .054 & .003 & .057 & 5.84 & 10.2 & .052 & & $<0.001$ \\
\hline
\end{tabular}




\begin{tabular}{|c|c|c|c|c|c|c|c|c|c|c|c|c|}
\hline \multirow{2}{*}{$\begin{array}{l}\text { SRM } 31 \mathrm{~mm} \\
\mathrm{D} \times 19 \mathrm{~mm} \\
\text { thick }\end{array}$} & \multirow{2}{*}{ Type } & \multicolumn{11}{|c|}{ Chemical Composition (Nominal Weight Percent) } \\
\hline & & $\mathrm{C}$ & $\mathrm{Mn}$ & $\mathbf{P}$ & $\mathbf{S}$ & $\mathrm{Si}$ & $\mathrm{Cu}$ & $\mathrm{Ni}$ & $\mathrm{Cr}$ & Mo & Co & $\mathrm{Fe}$ \\
\hline 1159 & $\mathrm{Ni48}$, balance $\mathrm{Fe}$ & 0.007 & 0.305 & 0.003 & 0.003 & 0.32 & 0.038 & 48.2 & 0.06 & 0.010 & 0.022 & 51.0 \\
\hline 1160 & 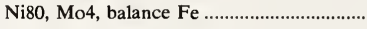 & .019 & .550 & .003 & .001 & .37 & .021 & 80.3 & .05 & 4.35 & .054 & 14.3 \\
\hline
\end{tabular}

Titanium-Base Alloys

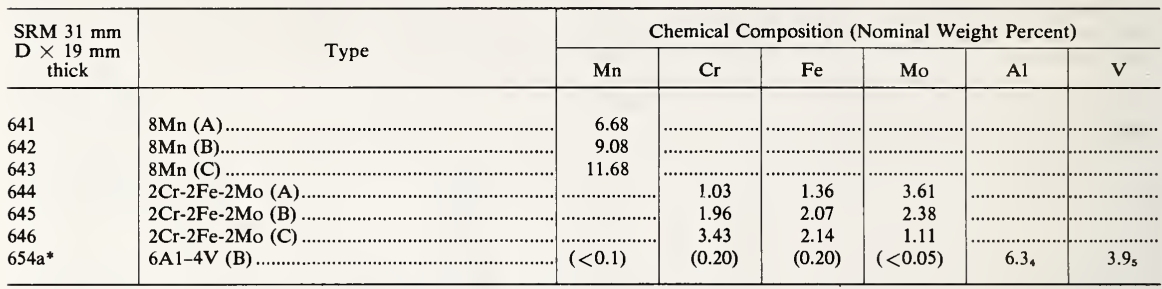

*31 mm D $\times 6.4 \mathrm{~mm}$ thick.

Zinc-Base Alloys

Zinc-base alloy SRM's are available ranging from very high-purity zinc to commercial materials such as spelter and die-casting alloy compositions. They are supplied as bar segments (disks) intended for calibrating and checking optical emission and $\mathrm{x}$-ray spectroscopic techniques. The certificate of analysis supplied with each gives the chemical composition determined at NBS and values determined by other laboratories that have cooperated in the certification of the SRM's. For high-purity Zinc, see High-Purity Metals, page 41 .

(Values in parentheses are not certified, but are given for information only.)

\begin{tabular}{|c|c|c|c|c|c|c|c|c|c|}
\hline \multirow{2}{*}{ SRM } & \multirow{2}{*}{ Type } & \multicolumn{8}{|c|}{ Chemical Composition (Nominal Weight Percent) } \\
\hline & & $\mathrm{Cu}$ & Al & $\mathrm{Mg}$ & $\mathrm{Fe}$ & $\mathrm{Pb}$ & $\mathrm{Cd}$ & Sn & $\mathrm{Cr}$ \\
\hline 625 & Zinc-base A-ASTM AG 40A & 0.034 & 3.06 & 0.070 & 0.036 & 0.0014 & 0.0007 & 0.0006 & 0.0128 \\
\hline 626 & Zinc-base B-ASTM AG 40A & .056 & 3.56 & .020 & .103 & .0022 & .0016 & .0012 & .0395 \\
\hline 627 & 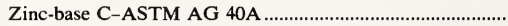 & .132 & 3.88 & .030 & .023 & .0082 & .0051 & .0042 & .0038 \\
\hline 628 & Zinc-base D-ASTM AC 41A & .611 & 4.59 & .0094 & .066 & .0045 & .0040 & .0017 & .0087 \\
\hline 629 & Zinc-base E-ASTM AC 41A & 1.50 & 5.15 & .094 & .017 & .0135 & .0155 & .012 & .0008 \\
\hline 630 & Zinc-base F-ASTM AC 41A & 0.976 & 4.30 & .030 & .023 & .0083 & .0048 & .0040 & .0031 \\
\hline 631 & Zinc spelter (modified) & .0013 & 0.50 & $(<001)$ & .005 & $(.001)$ & .0002 & .0001 & .0001 \\
\hline
\end{tabular}

\begin{tabular}{|c|c|c|c|c|c|c|c|c|}
\hline SRM & $\mathrm{Mn}$ & $\mathrm{Ni}$ & $\mathrm{Si}$ & In & $\mathrm{Ga}$ & $\mathrm{Ca}$ & $\mathrm{Ag}$ & $\mathrm{Ge}$ \\
\hline 625 & 0.031 & 0.0184 & 0.017 & & & & & \\
\hline 626 & .048 & .047 & .042 & ..................... & 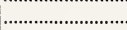 & 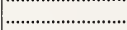 & ........ & ……………........... \\
\hline 627 & .014 & .0029 & .021 & - & 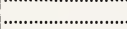 & 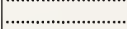 & ; & 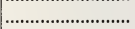 \\
\hline 628 & .0091 & .030 & .009 & 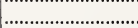 & 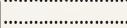 & 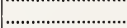 & & ………………..... \\
\hline 629 & .0017 & .0075 & .078 & . & 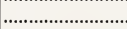 & ..................... & & ............................... \\
\hline 630 & .0106 & .0027 & .022 & & & & & \\
\hline 631 & .0015 & $(<.0005)$ & $<.002$ & $(0.0023)$ & $(0.002)$ & $<0.001$ & $(<0.0005)$ & $(0.0002)$ \\
\hline
\end{tabular}




\begin{tabular}{|c|c|c|c|c|c|c|c|c|c|c|c|c|c|c|}
\hline \multirow{2}{*}{$\begin{array}{c}\mathrm{SRM} 31 \mathrm{~mm} \\
\mathrm{D} \times 9.5 \mathrm{~mm} \\
\text { thick }\end{array}$} & \multirow{2}{*}{ Type } & \multicolumn{13}{|c|}{ Chemical Composition (Nominal Weight Percent) } \\
\hline & & Hf & C & $\mathrm{Cr}$ & $\mathrm{Cu}$ & $\mathrm{Fe}$ & Mn & Mo & $\mathrm{Ni}$ & $\mathrm{N}$ & $\mathrm{Si}$ & $\mathrm{Ti}$ & W & U \\
\hline $1212 \mathrm{a}$ & Zirconium $\mathrm{C}$..................... & & 0.28 & 0.063 & 0.015 & 0.071 & 0.030 & 0.012 & $0.043 \mathrm{~b}$ & b.012 & 0.035 & 0.015 & 0.014 & 0.010 \\
\hline 1234 & Unalloyed Zirconium A ......................... & 46 & $(80)$ & $(55)$ & $(<10)$ & $(240)$ & $(10)$ & (2) & (20) & (14) & $(40)$ & $(20)$ & $(25)$ & $\ldots . . . .$. \\
\hline 1235 & Unalloyed Zirconium B B............................ & 95 & (170) & (60) & $(80)$ & $(850)$ & $(25)$ & (40) & (65) & (32) & (95) & $(90)$ & $(50)$ & .......... \\
\hline 1236 & Unalloyed Zirconium C............................ & 198 & (240) & (250) & (250) & $(1700)$ & $(45)$ & (100) & (140) & (69) & $(205)$ & $(185)$ & $(140)$ & $\ldots \ldots$ \\
\hline 1237 & Zircaloy D & $|31|$ & $\mid(100)$ & $|(1510)|$ & $(<10)$ & $(1650)$ & $(10)$ & $(<10)$ & (40) & (19) & $\mid(35)$ & $(30)$ & (25) & ........... \\
\hline 1238 & Zircaloy E............................................ & 178 & (310) & | (580) & $(160)$ & $(2500)$ & $(60)$ & (120) & (100) & (72) & $(170)$ & $(100)$ & $(95)$ & ................ \\
\hline 1239 & 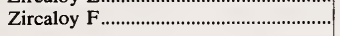 & 77 & $(170)$ & (1655) & (130) & $(2300)$ & $(50)$ & (45) & $(45)$ & $(42)$ & (95) & $(40)$ & $(45)$ & \\
\hline
\end{tabular}

\section{Gases in Metals}

\section{Certified for Hydrogen, Oxygen, and Nitrogen}

The SRM's are used in the determination of hydrogen, oxygen and nitrogen by vacuum fusion, inert gas fusion, and neutron activation methods. SRM's 1095 to 1099 were prepared from the same melt as the "1200" series (1261-1265), see page 24 . Values in parentheses are not certified; they are given for information only.

\begin{tabular}{|c|c|c|c|c|c|}
\hline SRM & Type & Form & $\begin{array}{c}\text { Oxygen } \\
(\mathrm{ppm})\end{array}$ & $\begin{array}{c}\text { Hydrogen } \\
\text { (ppm) }\end{array}$ & $\begin{array}{c}\text { Nitrogen } \\
\text { (ppm) }\end{array}$ \\
\hline $352 a$ & Unalloyed titanium for hydrogen... & Platelets... & & 20 & \\
\hline 354 & Unalloyed titanium for hydrogen.... & Platelets... & & 215 & \\
\hline 355 & 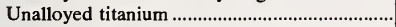 & 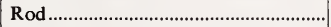 & 3031 & & \\
\hline 357 & Unalloyed Zirconium …………………………........... & Wire & (1200) & 19 & 49 \\
\hline 358 & Unalloyed Zirconium .................................................... & Wire & (1100) & 107 & 28 \\
\hline 1086 & 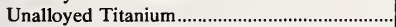 & 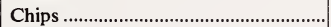 & (1350) & 116 & .................... \\
\hline 1087 & Unalloyed Titanium & 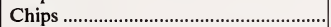 & $(840)$ & 57.5 & …………............ \\
\hline 1088 & 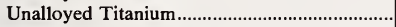 & 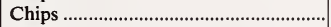 & (1450) & 88.5 & ......................... \\
\hline 1090 & Ingot iron & 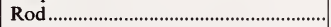 & 491 & & $(60)$ \\
\hline 1091 & Stainless steel (AISI 431) ........... & Rod........ & 131 & ...... & (945) \\
\hline 1092 & Vacuum-melted steel .......... & Rod.... & 28 & & (40) \\
\hline 1093 & Valve steel........................... & Rod........ & 60 & $\ldots \ldots$ & $(4807)$ \\
\hline 1094 & Maraging steel.... & Rod ........................ & 4.5 & .... & (71) \\
\hline${ }^{*} 1095$ & AISI 4340 steel......................... & Rod ................................................... & 9 & ……….......... & (37) \\
\hline *1096 & AISI 94B17 (Mod) steel........................................ & 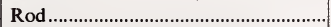 & 10.7 & ……………........ & 40.4 \\
\hline *1097 & Cr-V (Mod) steel ……………………………... & 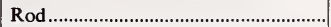 & 6.6 & ……………....... & (41) \\
\hline${ }^{*} 1098$ & High Carbon (Mod) steel ........................................ & 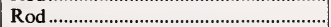 & 10 & ……................... & 32 \\
\hline *1099 & Electrolytic iron & 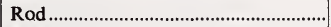 & 61 & ............................ & (13) \\
\hline 1089 & Set of $5: 1095,1096,1097,1098$, and $1099 \ldots \ldots$ & Rods & & .......................... & \\
\hline
\end{tabular}

*Sold only in sets as SRM 1089.

\section{High-Purity Metals}

Very high-purity metal SRM's are being made available to fill the needs of analysts determining impurity elements in high-purity metal materials. They are intended to serve as bench marks in calibration of methods and equipment; also, they are expected to be valuable in the development of new or improved methods and techniques for extending the sensitivity of detection in the determination of trace constituents 
in various materials by chemical, optical emission, solid mass spectroscopy, activation, and resistivity methods of analysis.

The Certificate of Analysis supplied with each high-purity SRM gives the state-of-the-art information on chemical composition in the cooperating laboratories for the various trace determinations reported.

High-purity gold is available in both wire and rod form. The wire form (W), is intended for applications such as spark source mass spectroscopic techniques. The low levels of impurities make it important for evaluating instrument and system blanks. The rod form (R), is intended for application in other methods of characterization.

Platinum is available in wire form as a high-purity material and as doped composition material.

Zinc is available in a high-purity and in a less pure version. Both were prepared from the same starting material. The high-purity material is the result of further purification by vacuum distillation, zone refining, and degasification. The zinc is supplied in the form of semi circular bar segments.

\begin{tabular}{|c|c|c|c|c|c|c|c|}
\hline \multirow[t]{2}{*}{ SRM } & \multirow[t]{2}{*}{ Type } & \multirow[t]{2}{*}{ Unit Size } & \multicolumn{5}{|c|}{$\begin{array}{c}\text { Chemical Compositions (Nominal Parts } \\
\text { Per Million by Weight) }\end{array}$} \\
\hline & & & $\mathrm{Cu}$ & $\mathrm{Ni}$ & Sn & $\mathrm{Pb}$ & $\mathrm{Zr}$ \\
\hline $685 W^{*}$ & High-Purity Gold (Wire) ...................... & $1.4 \mathrm{~mm} \mathrm{D} \times 102 \mathrm{~mm}$ long $\ldots \ldots \ldots \ldots \ldots \ldots \ldots \ldots \ldots \ldots \ldots$ & 0.1 & & & & \\
\hline $685 R^{*}$ & High-Purity Gold (Rod) ....................... & $5.9 \mathrm{~mm} \mathrm{D} \times 25 \mathrm{~mm}$ long $\ldots \ldots \ldots \ldots \ldots \ldots \ldots \ldots \ldots \ldots$ & .1 & & & & \\
\hline $680 \mathrm{aL} 1$ & High-Purity Platinum (Wire)................ & $0.51 \mathrm{~mm} \mathrm{D} \times 102 \mathrm{~mm}$ long $\ldots \ldots \ldots \ldots \ldots \ldots \ldots \ldots$ & .1 & $<1$ & & $<1$ & $<0.1$ \\
\hline $680 \mathrm{aL} 2$ & High-Purity Platinum (Wire)............... & 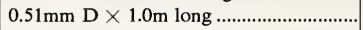 & .1 & $<1$ & ................... & $<1$ & $<0.1$ \\
\hline $681 \mathrm{~L} 1$ & Doped-Platinum (Wire)....................... & $0.51 \mathrm{~mm} \mathrm{D} \times 102 \mathrm{~mm}$ long $\ldots \ldots \ldots \ldots \ldots \ldots \ldots \ldots$ & 5.1 & 0.5 & (n................ & 12 & 11 \\
\hline $681 \mathrm{~L} 2$ & Doped-Platinum (Wire) ............................. & 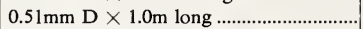 & 5.1 & 0.5 & & 12 & 11 \\
\hline $682^{*}$ & High-Purity Zinc ................................... & $\begin{array}{l}\text { Semicircular segments } 57 \mathrm{~mm} \mathrm{D} \\
\quad \times 19 \mathrm{~mm} \text { long. }\end{array}$ & 0.042 & & $(0.02)$ & & \\
\hline $683 *$ & 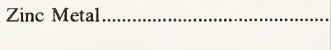 & $\begin{array}{l}\text { Semicircular segments } 57 \mathrm{~mm} \mathrm{D} \\
\times 19 \mathrm{~mm} \text { long. }\end{array}$ & 5.9 & & $(.02)$ & 11.1 & \\
\hline
\end{tabular}

\begin{tabular}{|c|c|c|c|c|c|c|c|c|c|c|c|}
\hline SRM & $\mathrm{Ag}$ & $\mathrm{Mg}$ & In & $\mathrm{Fe}$ & O & $\mathrm{Pd}$ & $\mathrm{Au}$ & $\mathrm{Rh}$ & Ir & $\mathrm{Cd}$ & $\mathrm{Ti}$ \\
\hline $685 \mathrm{~W} *$ & [0.1] & & 0.007 & 0.3 & [2] & & & & & & \\
\hline $685 R^{*}$ & [.1] & .................. & .007 & .2 & {$[<2]$} & & & & & & .... \\
\hline $680 \mathrm{aL} 1$ & $<.1$ & $<1$ & 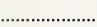 & 1.3 & 4 & 0.2 & $<1$ & $<0.2$ & $<0.01$ & & ............ \\
\hline $680 \mathrm{aL} 2$ & $<.1$ & $<1$ & ......................... & 1.3 & 4 & .2 & $<1$ & $<.2$ & .01 & ............. & ................. \\
\hline $681 \mathrm{~L} 1$ & 2.0 & 12 & $\ldots \ldots \ldots$ & 5 & 7 & 6 & 9 & 9 & 11 & & \\
\hline $681 \mathrm{~L} 2$ & 2.0 & 12 & $\ldots$ & 5 & 7 & 6 & 9 & 9 & 11 & & .................. \\
\hline $682^{*}$ & $(0.02)$ & . & 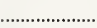 & (0.1) & & & & & & $(0.1)$ & \\
\hline 683* & 1.3 & 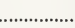 & 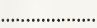 & 2.2 & & . & 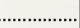 & ..................... & 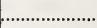 & 1.1 & $(0.2)$ \\
\hline
\end{tabular}

${ }^{*}$ Certificate gives upper limits for other elements found to be present.

\section{Microanalytical Standards}

These SRM's provide a highly homogeneous material at about the micrometer of spatial resolution. They are intended primarily for use in calibration of quantitative electron probe, secondary ion mass spectrometry, spark sources, mass spectrometry, and laser probe microanalytical techniques.

\section{Cartridge Brass}

Cartridge Brass, SRM 478, consists of two specimens: A chill-cast cube (6mm on edge) with a polished chill-cast face and a wrought right circular cylinder $(6 \mathrm{~mm}$ in diameter and height).

SRM 478 is homogeneous at micrometer levels of spatial resolution for both copper and zinc. Details of the homogeneity testing are in NBS Miscellaneous Publication 260-10. Extensive tests of SRM 478 with electron probe microanalyzers show that satisfactory analytical calibration can be performed using SRM 478. 
The Fe-Cr-Ni alloy, SRM 479a, is a wafer (4.6 mm in diameter and $1 \mathrm{~mm}$ thick) and is characterized for chemical homogeneity of iron, chromium, and nickel at the micrometer level of spatial resolution. It is satisfactory for use as a homogeneous material for electron probe microanalysis.

\section{Tunsten-20\% Molybdenum}

The tunsten-20\% molybdenum alloy, SRM 480, is a wafer ( $1 \mathrm{~mm}$ in diameter and $1 \mathrm{~mm}$ thick) with a core of tunsten-20\% molybdenum wire embedded in pure molybdenum onto which pure tungsten has been deposited by electroplating to provide a composite. Details on homogeneity characterization are given in NBS Spec. Publ. 260-16. (See inside back cover for ordering instructions.)

\section{Gold-Silver}

Six color-coded wires $(0.5 \mathrm{~mm}$ in diameter and $50 \mathrm{~mm}$ long) comprise SRM 481 . The wires consist of a high-purity gold and a high-purity silver wire and four wires with nominal chemical composition differences in steps of $20 \%$.

\section{Gold-Copper}

Six color-coded wires $(0.5 \mathrm{~mm}$ in diameter and $50 \mathrm{~mm}$ long) comprise SRM 482 , which is similar to the gold-silver set. In both sets special precautions were taken to achieve homogeneity on a microscopic scale.

\section{Iron-3\% Silicon}

The iron-3\% silicon microprobe, SRM 483, is a platelet ( $3 \mathrm{~mm} \times 3 \mathrm{~mm} \times 0.28 \mathrm{~mm}$ ), and is characterized for chemical homogeneity of iron and silicon at the micrometer level of spatial resolution. It is satisfactory for use as a homogeneous material for electron probe microanalysis.

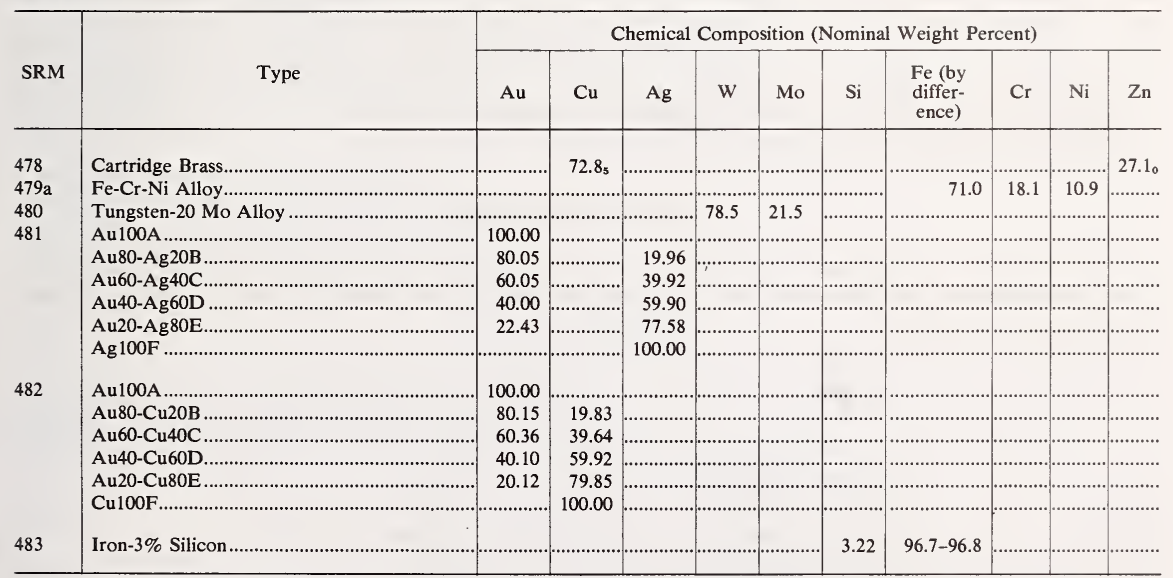


These SRM's are high-purity chemicals defined as primary, working, and secondary standards in accordance with recommendations of the Analytical Chemistry Section of the International Union of Pure and Applied Chemistry [Ref. Analyst 90, 251 (1965)]. These definitions are as follows:

Primary Standard:

a commercially available substance of purity $100 \pm 0.02$ percent (Purity $99.98+$ percent).

Working Standard:

a commercially available substance of purity $100 \pm 0.05$ percent (Purity $99.95+$ percent).

Secondary Standard:

a substance of lower purity which can be standardized against a primary grade standard.

\begin{tabular}{|c|c|c|c|c|}
\hline SRM & Type & $\begin{array}{l}\text { Wt/Unit } \\
\text { (grams) }\end{array}$ & Certified Use & $\begin{array}{l}\text { Purity } \\
\text { Stoichio- } \\
\text { metric }\end{array}$ \\
\hline $17 \mathrm{c}$ & Sucrose & 60 & 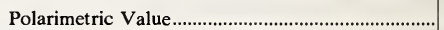 & $\left({ }^{a}\right)$ \\
\hline $40 \mathrm{~h}$ & 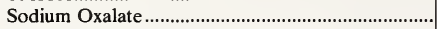 & 60 & 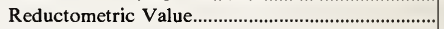 & 99.95 \\
\hline $41 \mathrm{~b}$ & Dextrose (D-glucose) & 70 & 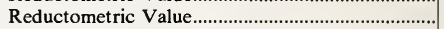 & \\
\hline $83 d$ & 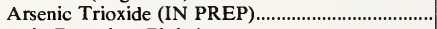 & (...................... & Reductometric Value................................... & \\
\hline $84 \mathrm{j}$ & Acid Potassium Phthalate ............................................. & 60 & Acidimetric Value................................... & 99.996 \\
\hline $136 \mathrm{c}$ & Potassium Dichromate & 60 & Oxidimetric Value & 99.98 \\
\hline $350 \mathrm{a}$ & 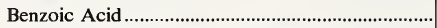 & 30 & 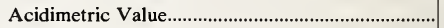 & 99.99 \\
\hline $723 a$ & 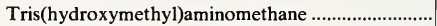 & 50 & Basimetric Value & 99.97 \\
\hline 944 & 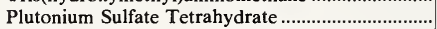 & 0.5 & Assay & 100.00 \\
\hline $949 \mathrm{e}$ & 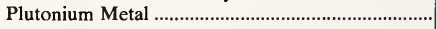 & 0.5 & Assay & 99.996 \\
\hline $950 \mathrm{~b}$ & Uranium Oxide $\left(\mathrm{U}_{3} \mathrm{O}_{8}\right)$ & 25 & 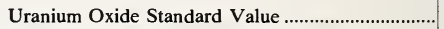 & 99.968 \\
\hline 951 & Boric Acid & 100 & Acidimetric and Boron Isotopic Value.................... & 100.00 \\
\hline 960 & Uranium Metal & 26 & Assay & 99.975 \\
\hline 984 & Rubidium Chloride & 0.25 & 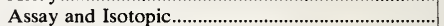 & 99.90 \\
\hline 985 & Potassium Chloride & 1 & Assay and Isotopic & 99.99 \\
\hline 987 & Strontium Carbonate & 1 & 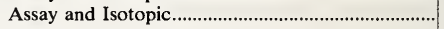 & 99.98 \\
\hline \multirow[t]{3}{*}{999} & Potassium Chloride & 60 & Assay Standard for: & \\
\hline & & & Potassium & 99.98 \\
\hline & & & Chloride & 99.99 \\
\hline
\end{tabular}

Sucrose $=$ Moisture $<0.01$ percent, Reducing Substances $<0.02$ percent, Ash 0.001 percent.

b Dextrose $=$ Moisture 0.07 percent, Ash 0.002 percent.

\section{Microchemical Standards}

These SRM's are furnished as fine crystals of suitable homogeneity for use as standards for conventional microchemical methods of analysis employing samples of approximately $5 \mathrm{mg}$. See also Microanalytical Standards, page 42.

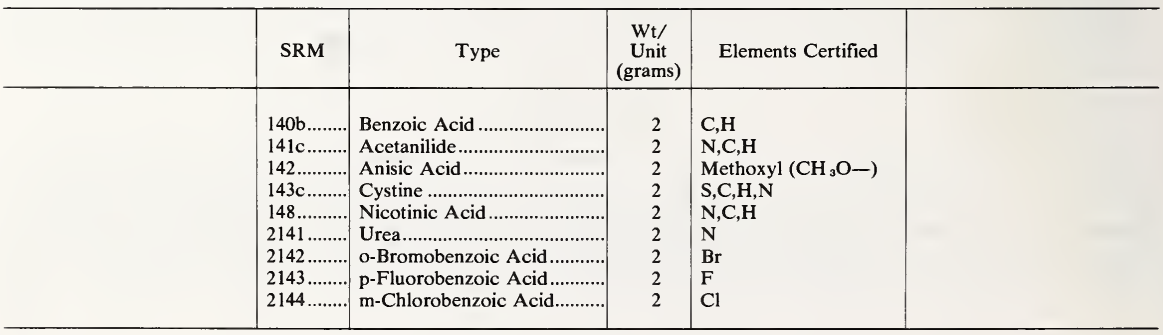


These SRM's are intended for use in calibrating apparatus and validating analytical methods used in clinical and pathological laboratories, and to assist manufacturers of clinical products in meeting the chemical and physical specifications required for clinical chemicals. (For details on SRM's 930D and 93lc, see Spectrophotometric Filters, page 75.)

\begin{tabular}{|c|c|c|c|c|}
\hline SRM & Type & $\begin{array}{c}\text { Associated } \\
\text { NBS } \\
\text { Publications }\end{array}$ & Purity \% & Wt/Unit \\
\hline 900 & 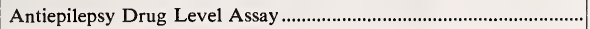 & ........................ & 4 drugs $/ 3$ levels & Set of 4 vials \\
\hline 909 & Human Serum & 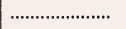 & 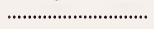 & . \\
\hline 910 & Sodium Pyruvate (IN PREP) & ......................... & (n............................. & . \\
\hline $911 \mathrm{a}$ & 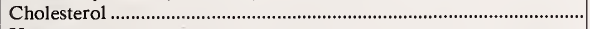 & ....................... & 99.8 & $2 \mathrm{~g}$ \\
\hline $912 \mathrm{a}$ & Urea & (....................... & 99.8 & $25 \mathrm{~g}$ \\
\hline 913 & 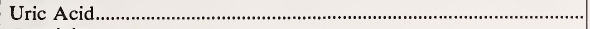 & ......................... & 99.7 & $10 \mathrm{~g}$ \\
\hline 914 & 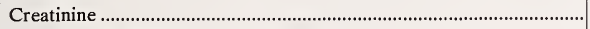 & ......................... & 99.8 & $10 \mathrm{~g}$ \\
\hline 915 & Calcum Carbonate & SP $260-36$ & 99.9 & $20 \mathrm{~g}$ \\
\hline 916 & Bilirubin na. & ......................... & 99.0 & $100 \mathrm{mg}$ \\
\hline 917 & 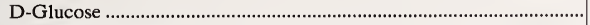 & ........................ & 99.9 & $25 \mathrm{~g}$ \\
\hline 918 & Potassium Chloride & SP 260-63 & 99.9 & $30 \mathrm{~g}$ \\
\hline 919 & Sodium Chloride & SP $260-60$ & 99.9 & $30 \mathrm{~g}$ \\
\hline 920 & D-Mannitol & ........................ & 99.8 & $50 \mathrm{~g}$ \\
\hline 921 & Cortisol & ........................ & 98.9 & $1 \mathrm{~g}$ \\
\hline 922 & Tris (hydroxymethyl) aminomethane & ........................ & 99.9 & $25 \mathrm{~g}$ \\
\hline 923 & Tris (hydroxymethyl) aminomethane $\mathrm{HCl}$ & ..................... & 99.7 & $35 \mathrm{~g}$ \\
\hline 924 & Lithium Carbonate & SP $260-70$ & 100.0 & $30 \mathrm{~g}$ \\
\hline 925 & 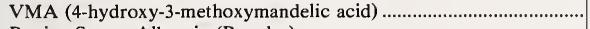 & ........................ & 99.4 & $1 \mathrm{~g}$ \\
\hline 926 & Bovine Serum Albumin (Powder) & ....................... & ** & $5 \mathrm{~g}$ \\
\hline 927 & Bovine Serum Albumin ( $7 \%$ Solution) & ......................... & ** & 10 vials, $2.15 \mathrm{~mL}$ ea. \\
\hline 928 & Lead Nitrate & . & 100.00 & $30 \mathrm{~g}$ \\
\hline 929 & 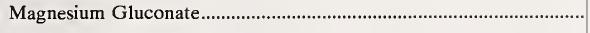 & ............................ & 100.1 & $5 \mathrm{~g}$ \\
\hline 930D & Glass Filters for Spectrophotometry & SP 260-51 & + & Set of 3 \\
\hline $931 \mathrm{c}$ & 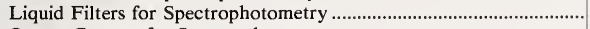 & ........................ & + & 3 sets of 4 \\
\hline 932 & 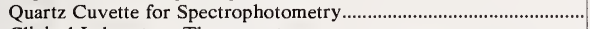 & SP $260-32$ & + & 1 each \\
\hline 934 & 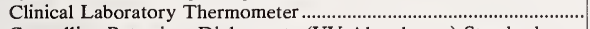 & SP 260-48 & 计 & 1 each \\
\hline 935 & Crystalline Potassium Dichromate (UV Absorbance) Standard ........ & SP 260-54 & $(99.972)^{* * *}$ & $15 \mathrm{~g}$ \\
\hline 936 & 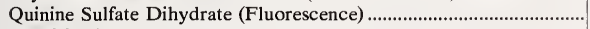 & SP 260-64 & $(98.2)^{* * *}$ & $1 \mathrm{~g}$ \\
\hline 937 & 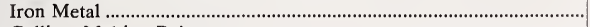 & ........................ & 99.90 & $50 \mathrm{~g}$ \\
\hline 1968 & 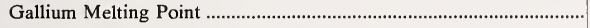 & SP 481 & +++ & 1 ea. \\
\hline
\end{tabular}

+ Certified for optical properties (see p. 76.)

++ Individually calibrated at $0,25,30$, and $37^{\circ} \mathrm{C}$.

**Conforms to NCCLS specification ACC-1.

***Apparent purity, certified for optical properties.

+++ Melting Point Certified at $29.7723^{\circ} \mathrm{C}$. (See p. 71.)

\section{Biological Standards}

These SRM's are intended for use in the calibration of apparatus and methods used in the analysis of biological materials for major, minor, and trace constituents.

(Values in parentheses are not certified, but are given for information only.)

\begin{tabular}{|c|c|c|c|c|c|}
\hline SRM & Type & $\begin{array}{l}\text { Wt/Unit } \\
\text { (grams) }\end{array}$ & SRM & Type & $\begin{array}{l}\text { Wt/Unit } \\
\text { (grams) }\end{array}$ \\
\hline 1566 & 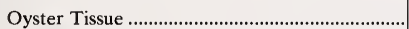 & 30 & 1572 & Citrus Leaves (IN PREP).................... & \\
\hline 1567 & Wheat Flour & 80 & 1573 & Tomato Leaves & 70 \\
\hline 1568 & Rice Flour & 80 & 1575 & 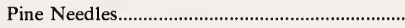 & 70 \\
\hline 1569 & 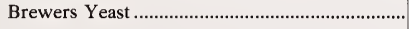 & 50 & $1577 \mathrm{a}$ & 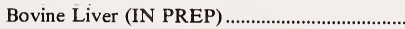 & 50. \\
\hline
\end{tabular}




\begin{tabular}{|c|c|c|c|c|c|c|c|}
\hline \multirow{2}{*}{ Element } & \multirow{2}{*}{ SRM } & \multicolumn{6}{|c|}{ Content in $\mu \mathrm{g} / \mathrm{g}$ (or where noted, wt $\%$ ) } \\
\hline & & 1566 & 1567 & 1568 & 1569 & 1573 & 1575 \\
\hline \multirow{2}{*}{\multicolumn{8}{|c|}{ Aluminum }} \\
\hline & & & & & & & $(0.2)$ \\
\hline 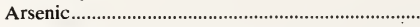 & ............... & 13.41 & $(0.006)$ & 0.41 & ........... & 0.27 & 0.21 \\
\hline 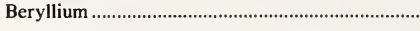 & & .......................... & ........................ & . & ............. & ....................... & ......................... \\
\hline \multicolumn{8}{|l|}{ Boron ........ } \\
\hline Bromine & ............... & \multirow{4}{*}{$\begin{array}{l}\text { (55) } \\
\quad 3.5 \\
0.15 \%\end{array}$} & \multirow{4}{*}{$\begin{array}{l}\text { (9) } \\
0.032 \\
0.019 \%\end{array}$} & \multirow{4}{*}{$\begin{array}{l}\text { (1) } \\
0.029 \\
0.014 \%\end{array}$} & .......... & (26) & \multirow{5}{*}{$\begin{array}{l}(9) \\
(<0.5) \\
0.41 \% \\
(0.4)\end{array}$} \\
\hline 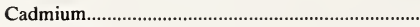 & .............. & & & & & & \\
\hline 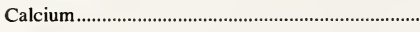 & ............. & & & & .................. & $3.00 \%$ & \\
\hline \multicolumn{2}{|l|}{ Cerium } & & & & & (1.6) & \\
\hline 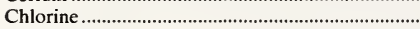 & ………....... & \multirow{3}{*}{$\begin{array}{c}(1.0 \%) \\
0.69 \\
(0.4)\end{array}$} & .......................... & ……................... & ........... & \multirow{3}{*}{$\begin{array}{c}4.5 \\
(0.6)\end{array}$} & \\
\hline 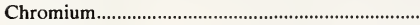 & ............... & & ............................ & ……………....... & 2.12 & & \multirow{2}{*}{$\begin{array}{c}2.6 \\
(0.1)\end{array}$} \\
\hline 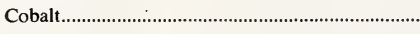 & ..................... & & {$[\ldots \ldots \ldots \ldots \ldots \ldots \ldots$} & 0.02 & & & \\
\hline \multirow{2}{*}{ Copper } & & \multirow[t]{2}{*}{63.0} & \multirow{2}{*}{2.0} & \multirow[t]{2}{*}{2.2} & & \multirow{2}{*}{$\begin{array}{l}11 \\
(0.04)\end{array}$} & \multirow{2}{*}{$\begin{array}{l}3.0 \\
(0.006)\end{array}$} \\
\hline & & & & & ............ & & \\
\hline Indium & & & ... & ........ & ........ & & 年 \\
\hline \multicolumn{2}{|l|}{ Iodine... } & \multirow{3}{*}{$\begin{array}{l}(2.8) \\
195\end{array}$} & & & & & \\
\hline Iron ...................... & ............ & & 18.3 & 8.7 & ........... & \multirow{3}{*}{$\begin{array}{c}690 \\
(0.9) \\
6.3\end{array}$} & \multirow{3}{*}{$\begin{array}{l}200 \\
(0.2) \\
10.8\end{array}$} \\
\hline Lanthanum...... & & & & & & & \\
\hline 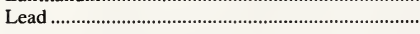 & .......... & \multirow{7}{*}{$\begin{array}{c}0.48 \\
.128 \% \\
17.5 \\
0.057 \\
(\leqslant 0.2) \\
1.03\end{array}$} & . & & ........... & & \\
\hline 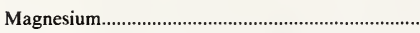 & ........... & & & & & $(0.7 \%)$ & \\
\hline 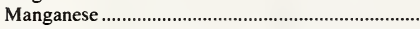 & ............... & & & 20.1 & ............. & & 675 \\
\hline 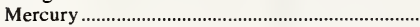 & .................. & & 0.001 & 0.0060 & .............. & $(0.1)$ & 0.15 \\
\hline Molybdenum & ................ & & $(0.4)$ & (1.6) & ............. & ........................ & \\
\hline 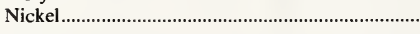 & ................. & & & $(0.16)$ & .1.n. & (n) & (3.5) \\
\hline Nitrogen............ & & & & & & $(5.0 \%)$ & $(1.2 \%)$ \\
\hline Phosphorus & ............... & $(0.81 \%)$ & & & ......... & $0.34 \%$ & $0.12 \%$ \\
\hline Potassium & .............. & $0.969 \%$ & $0.136 \%$ & $0.112 \%$ & ................ & $4.46 \%$ & $0.37 \%$ \\
\hline Rubidium & ............... & 4.45 & & & .................. & 16.5 & 11.7 \\
\hline Scandium & 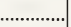 & & & & .......... & $(0.13)$ & $(0.03)$ \\
\hline Selenium . & & 2.1 & 1.1 & 0.4 & & & \\
\hline Silicon ... & & & & & & & ...... $>{ }^{\prime}$ \\
\hline Silver................................... & .............. & 0.89 & & & & & 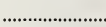 \\
\hline Sodium ........................................ & .............. & $0.51 \%$ & 8.0 & 6.0 & & & \\
\hline 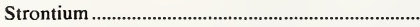 & ........... & 10.36 & & & & 44.9 & 4.8 \\
\hline Sulfu & & $(.076 \%)$ & & & & & \\
\hline Tellurium ............... & & & $(\geqslant 0.002)$ & $(\geqslant 0.002)$ & & & ………............... \\
\hline Thallium..................................... & ............ & $(\leqslant 0.005)$ & & & & $(0.05)$ & $(0.05)$ \\
\hline Thorium ...................................... & & $(0.1)$ & 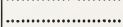 & ............ & & 0.17 & 0.037 \\
\hline Uranium & .......... & 0.116 & ........... & 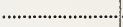 & ............... & 0.061 & 0.020 \\
\hline Zinc..... & & 852 & 10.6 & 19.4 & & 62 & \\
\hline
\end{tabular}




\section{Environmental Standards}

\section{Analyzed Gases}

These SRM's are intended for the calibration of apparatus used for the measurement of various components in gas mixtures, and in some cases for particular atmospheric pollutants. Each SRM is accurately certified and is primarily intended to monitor and correct for long-term drifts in instruments used. Each cylinder contained 870 liters at STP prior to certification, and thus contains somewhat less than 870 L. All cylinders conform to the appropriate DOT specifications.

\begin{tabular}{|c|c|c|}
\hline SRM & Type & Nominal Concentrations \\
\hline $1658 \mathrm{a}$ & Methane in Air & $\mathrm{CH}_{4}, 0.951 \mu \mathrm{mol} / \mathrm{mol}(\mathrm{ppm})$. \\
\hline $1659 \mathrm{a}$ & Methane in Air & $\mathrm{CH}_{4}, 9.43 \mu \mathrm{mol} / \mathrm{mol}(\mathrm{ppm})$ \\
\hline $1660 \mathrm{a}$ & 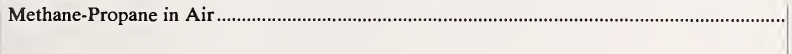 & $\begin{array}{l}\mathrm{CH}_{4}, 4.10 \mu \mathrm{mol} / \mathrm{mol}(\mathrm{ppm}) . \\
\mathrm{C}_{3} \mathrm{H}_{8}, 0.976 \mu \mathrm{mol} / \mathrm{mol}(\mathrm{ppm}) .\end{array}$ \\
\hline 1661 & 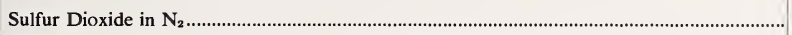 & $\mathrm{SO}_{2}, 480 \mu \mathrm{mol} / \mathrm{mol}(\mathrm{ppm})$ \\
\hline $1662 \mathrm{a}$ & Sulfur Dioxide in $\mathrm{N}_{2}$ & $\mathrm{SO}_{2}, 942 \mu \mathrm{mol} / \mathrm{mol}(\mathrm{ppm})$ \\
\hline $1663 a$ & Sulfur Dioxide in $\mathrm{N}_{2}$ & $\mathrm{SO}_{2}, 1497 \mu \mathrm{mol} / \mathrm{mol}(\mathrm{ppm})$ \\
\hline 1664 & Sulfur Dioxide in $\mathrm{N}_{2}$ & $\mathrm{SO}_{2}, 2521 \mu \mathrm{mol} / \mathrm{mol}(\mathrm{ppm})$ \\
\hline $1665 \mathrm{~b}$ & ( & $\mathrm{C}_{3} \mathrm{H}_{8}, 3 \mathrm{ppm}$. \\
\hline $1666 \mathrm{~b}$ & Propane in Air & $\mathrm{C}_{3} \mathrm{H}_{8}, 10 \mathrm{ppm}$. \\
\hline $1667 \mathrm{~b}$ & 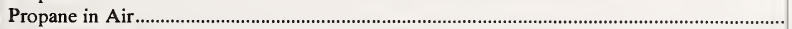 & $\mathrm{C}_{3} \mathrm{H}_{8}, 50 \mathrm{ppm}$. \\
\hline $1668 \mathrm{~b}$ & Propane in Air & $\mathrm{C}_{3} \mathrm{H}_{8}, 100 \mathrm{ppm}$. \\
\hline $1669 \mathrm{~b}$ & Propane in Air & $\mathrm{C}_{3} \mathrm{H}_{8}, 500 \mathrm{ppm}$ \\
\hline $1674 \mathrm{~b}$ & Carbon Dioxide in Nitrogen & $\mathrm{CO}_{2}, 7.5 \mathrm{~mol} \%$. \\
\hline $1675 b$ & 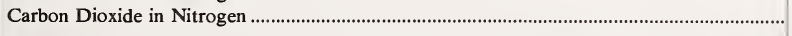 & $\mathrm{CO}_{2}, 15.0 \mathrm{~mol} \%$ \\
\hline $1677 \mathrm{c}$ & Carbon Monoxide in Nitrogen & $\mathrm{CO}, 10 \mathrm{ppm}$. \\
\hline $1678 \mathrm{c}$ & Carbon Monoxide in Nitrogen & $\mathrm{CO}, 50 \mathrm{ppm}$. \\
\hline $1679 \mathrm{c}$ & 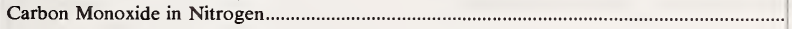 & $\mathrm{CO}, 100 \mathrm{ppm}$. \\
\hline $1680 \mathrm{~b}$ & Carbon Monoxide in Nitrogen & $\mathrm{CO}, 500 \mathrm{ppm}$. \\
\hline $1681 \mathrm{~b}$ & 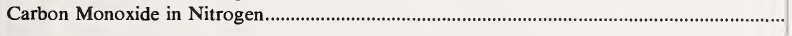 & $\mathrm{CO}, 1000$ ppm. \\
\hline $1683 a$ & Nitric Oxide in Nitrogen .... & NO, 50 ppm. \\
\hline $1684 \mathrm{a}$ & Nitric Oxide in Nitrogen & NO, 100 ppm. \\
\hline $1685 \mathrm{a}$ & Nitric Oxide in Nitrogen & NO, 250 ppm. \\
\hline $1686 a$ & Nitric Oxide in Nitrogen & NO, $500 \mathrm{ppm}$. \\
\hline $1687 \mathrm{a}$ & 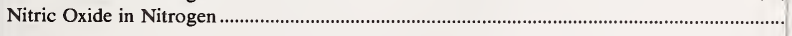 & NO, 1000 ppm. \\
\hline $2612 \mathrm{a}$ & Carbon Monoxide in Air & $\mathrm{CO}, 10 \mu \mathrm{mol} / \mathrm{mol}(\mathrm{ppm})$ \\
\hline $2613 a$ & 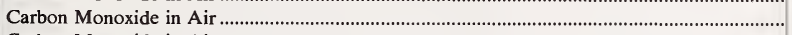 & $\mathrm{CO}, 18.1 \mu \mathrm{mol} / \mathrm{mol}(\mathrm{ppm})$. \\
\hline $2614 a$ & Carbon Monoxide in Air & $\mathrm{CO}, 43.0 \mu \mathrm{mol} / \mathrm{mol}(\mathrm{ppm})$ \\
\hline $2619 a$ & Carbon Dioxide in $\mathrm{N}_{2}$ & $\mathrm{CO}_{2}, 0.5 \mathrm{~mol}$ percent. \\
\hline $2620 \mathrm{a}$ & Carbon Dioxide in $\mathrm{N}_{2}$ & $\mathrm{CO}_{2}, 1.0 \mathrm{~mol}$ percent. \\
\hline 2621a & 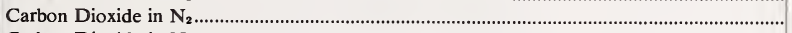 & $\mathrm{CO}_{2}, 1.5 \mathrm{~mol}$ percent. \\
\hline $2622 \mathrm{a}$ & Carbon Dioxide in $\mathrm{N}_{2}$ & $\mathrm{CO}_{2}, 2.0 \mathrm{~mol}$ percent. \\
\hline $2623 a$ & Carbon Dioxide in $\mathrm{N}_{2}$ & $\mathrm{CO}_{2}, 2.5 \mathrm{~mol}$ percent. \\
\hline 2624a & 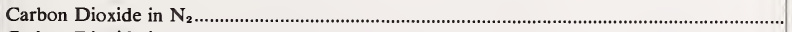 & $\mathrm{CO}_{2}, 3.0 \mathrm{~mol}$ percent. \\
\hline $2625 \mathrm{a}$ & 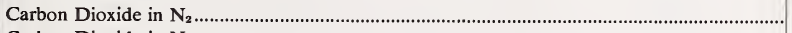 & $\mathrm{CO}_{2}, 3.5 \mathrm{~mol}$ percent. \\
\hline $2626 a$ & Carbon Dioxide in $\mathrm{N}_{2}$ & $\mathrm{CO}_{2}, 4.0 \mathrm{~mol}$ percent. \\
\hline
\end{tabular}




\begin{tabular}{|c|c|c|}
\hline SRM & Type & Nominal Concentrations \\
\hline $\begin{array}{l}2630 \\
2631\end{array}$ & $\begin{array}{l}\text { Nitric Oxide in } \mathrm{N}_{2} \\
\text { Nitric Oxide in } \mathrm{N}^{2}\end{array}$ & $\mathrm{NO}, 1500 \mu \mathrm{mol} / \mathrm{mol}(\mathrm{ppm})$. \\
\hline 2031 & 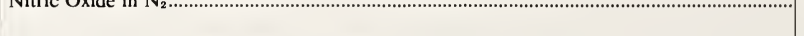 & $\mathrm{NO}, 3000 \mu \mathrm{mol} / \mathrm{mol}(\mathrm{ppm})$ \\
\hline 2632 & Carbon Dioxide in $\mathrm{N}_{2}$ & $\mathrm{CO}_{2} 300 \mu \mathrm{mol} / \mathrm{mol}(\mathrm{ppm})$ \\
\hline 2633 & Carbon Dioxide in $\mathrm{N}_{2}$ & $\mathrm{CO}_{2} 400 \mu \mathrm{mol} / \mathrm{mol}(\mathrm{ppm})$ \\
\hline 2634 & 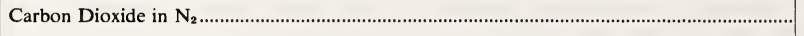 & $\mathrm{CO}_{2} 800 \mu \mathrm{mol} / \mathrm{mol}(\mathrm{ppm})$. \\
\hline 2635 & Carbon Monoxide in $\mathrm{N}_{2}$ & $\mathrm{CO}, 25 \mu \mathrm{mol} / \mathrm{mol}(\mathrm{ppm})$ \\
\hline 2636 & Carbon Monoxide in $\mathrm{N}_{2}$ & $\mathrm{CO}, 250 \mu \mathrm{mol} / \mathrm{mol}(\mathrm{ppm})$ \\
\hline 2637 & Carbon Monoxide in $\mathrm{N}_{2}$ & $\mathrm{CO}, 2500 \mu \mathrm{mol} / \mathrm{mol}(\mathrm{ppm})$ \\
\hline 2638 & Carbon Monoxide in $\mathrm{N}_{2}$ & $\mathrm{CO}, 5000 \mu \mathrm{mol} / \mathrm{mol}(\mathrm{ppm})$ \\
\hline 2639 & Carbon Monoxide in $\mathrm{N}_{2}$ & $\mathrm{CO}, 1 \mathrm{~mol} \%$ \\
\hline 2640 & Carbon Monoxide in $\mathrm{N}_{2}$ & $\mathrm{CO}, 2 \mathrm{~mol} \%$. \\
\hline 2641 & Carbon Monoxide in $\mathrm{N}_{2}$ & $\mathrm{CO}, 4 \mathrm{~mol} \%$. \\
\hline 2642 & Carbon Monoxide in $\mathrm{N}_{2}$ & $\mathrm{CO}, 8 \mathrm{~mol} \mathrm{\%}$. \\
\hline 2657 & Oxygen in Nitrogen & $\mathrm{O}_{2}, 2 \mathrm{~mol} \%$ \\
\hline 2658 & Oxygen in Nitrogen & $\mathrm{O}_{2}, 10 \mathrm{~mol} \mathrm{\%}$. \\
\hline
\end{tabular}

\section{Analyzed Liquids and Solids}

These SRM's are intended for use in the analysis of materials for constituents of interest in health or environmental problems. See also: Clinical SRM's page 45, and Industrial Hygiene SRM's page 51.

\section{SINGLE ELEMENT Concentrations: \\ Weight percent-boldface \\ Microgram per gram-light face \\ Nanogram per milliliter-italics}

\begin{tabular}{|c|c|c|c|c|c|c|c|}
\hline SRM & \multicolumn{2}{|l|}{ Type } & \multicolumn{2}{|l|}{ Unit Size } & Lead & Sulfur & \multirow{10}{*}{$\begin{array}{l}\ldots \ldots \ldots \ldots \ldots . . . \\
\ldots .13 \mu \mathrm{g} / \mathrm{g} . \\
1.10 \mu \mathrm{g} / \mathrm{mL} \\
1.10 \mathrm{ng} / \mathrm{mL} .\end{array}$} \\
\hline 1579 & \multirow{5}{*}{\multicolumn{2}{|c|}{ 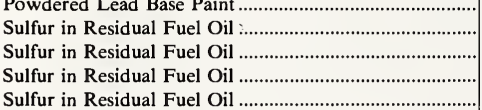 }} & \multirow{2}{*}{\multicolumn{2}{|c|}{$35 \mathrm{~g}$}} & $11.87 \%$ & \multirow[b]{2}{*}{$4.48 \%$} & \\
\hline 1620 & & & & & & & \\
\hline $1621 \mathrm{a}$ & & & & & $0.94 \%$ & \\
\hline $1622 \mathrm{a}$ & & & \multicolumn{2}{|c|}{$100 \mathrm{~mL}$} & & \multirow{2}{*}{$\begin{array}{l}0.94 \% \\
1.96 \%\end{array}$} & \\
\hline $1623 a$ & & & & & & \\
\hline 1624 & \multicolumn{2}{|l|}{ 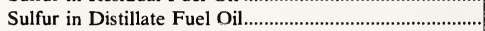 } & \multicolumn{2}{|c|}{ IN PREP } & & & \\
\hline 1630 & \multicolumn{2}{|l|}{ 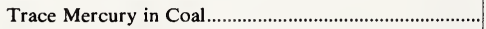 } & \multicolumn{2}{|c|}{$50 \mathrm{~g}$} & & & \\
\hline $1641 \mathrm{a}$ & \multicolumn{2}{|l|}{ Mercury in Water $(\mu \mathrm{g} / \mathrm{mL})$} & & & & \\
\hline $1642 \mathrm{a}$ & \multicolumn{2}{|l|}{ Mercury in Water $(\mathrm{ng} / \mathrm{mL})$} & \multicolumn{2}{|c|}{$950 \mathrm{~mL}$} & & & \\
\hline SRM & Type & \multicolumn{2}{|r|}{ Element Certified } & \multicolumn{3}{|c|}{ Nominal Concentration } & No. Units \\
\hline $1636 \mathrm{a}$ & 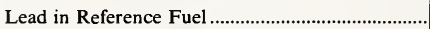 & \multirow{3}{*}{\multicolumn{2}{|c|}{$\begin{array}{l}\mathrm{Pb} \\
\mathrm{Pb} \\
\mathrm{Pb}\end{array}$}} & \multirow{3}{*}{\multicolumn{3}{|c|}{ 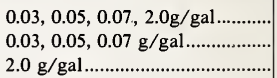 }} & \multirow{3}{*}{$\begin{array}{l}3 \text { vials each. } \\
4 \text { vials each. } \\
12 \text { vials each. }\end{array}$} \\
\hline $1637 a$ & 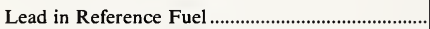 & & & & & & \\
\hline $1638 \mathrm{a}$ & 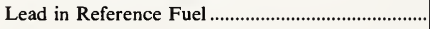 & & & & & & \\
\hline
\end{tabular}


MULTI-ELEMENT Concentrations:

Weight percent-boldface

Microgram per gram-light face

Nanogram per gram-italics

\begin{tabular}{|c|c|c|c|c|c|c|c|c|}
\hline SRM & Type & Unit size & A1 & $\mathrm{Sb}$ & As & $\mathbf{B a}$ & $\mathrm{Be}$ & $\mathrm{Br}$ \\
\hline $1632 a$ & $\begin{array}{l}\text { Trace Elements in Coal (Bitumi- } \\
\text { nous). }\end{array}$ & $75 \mathrm{~g}$ & (3.1) & $(0.6)$ & 9.3 & & & \\
\hline $1633 a$ & Trace Elements in Coal Fly Ash... & $75 \mathrm{~g}$ & (14) & (7) & 145 & (0.15) & $(12)$ & \\
\hline 1634 & Trace Elements in Fuel Oil ................ & 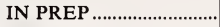 & & & & & & \\
\hline 1635 & $\begin{array}{l}\text { Trace Elements in Coal (Subbitu- } \\
\text { minous). }\end{array}$ & $75 \mathrm{~g} \ldots \ldots \ldots \ldots \ldots \ldots \ldots$ & $(0.32)$ & $(0.14)$ & 0.42 & & & \\
\hline $1643 a$ & Trace Elements in Water $(\mathrm{ng} / \mathrm{g}) . .$. & $950 \mathrm{~mL}$ & & & 76 & 46 & 19 & \\
\hline 1645 & 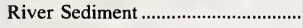 & $70 \mathrm{~g}$ & . & $(51)$ & $(66)$ & & & \\
\hline 1646 & 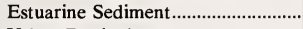 & IN PREP & & & & & & \\
\hline 1648 & 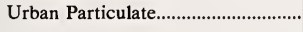 & 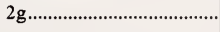 & (3.3) & $(45)$ & 115 & (737) & & $(500)$ \\
\hline
\end{tabular}

\begin{tabular}{|c|c|c|c|c|c|c|}
\hline SRM & $\mathrm{Cd}$ & $\mathrm{Ca}$ & $\mathrm{Ce}$ & Cs & $\mathrm{Cl}$ & $\mathrm{Cr}$ \\
\hline $1632 a$ & 0.17 & 0.23 & $(30)$ & $(2.4)$ & & 34.4 \\
\hline $1633 a$ & 1.0 & 1.11 & (180) & (11) & ...... & 196 \\
\hline $1634 a$ & & & & & & \\
\hline 1635 & 0.03 & (1) & (3.6) & & & 2.5 \\
\hline $1643 a$ & 10 & . & 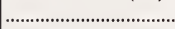 & 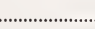 & & 17 \\
\hline 1645 & 10.2 & (.). & 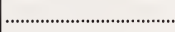 & (n).................. & 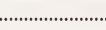 & 2.96 \\
\hline 1646 & ............... & 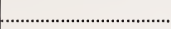 & 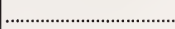 & ........................ & .......................... & (....................... \\
\hline 1648 & 75 & . & $(55)$ & (3) & (0.45) & 403 \\
\hline
\end{tabular}

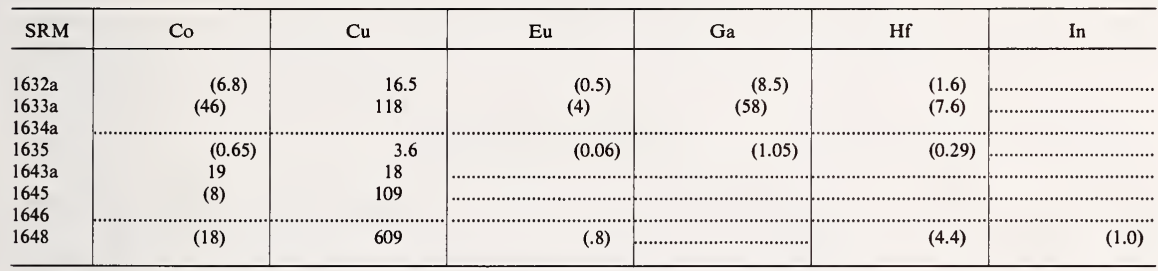

\begin{tabular}{|c|c|c|c|c|c|c|}
\hline SRM & I & $\mathrm{Fe}$ & $\mathrm{La}$ & $\mathrm{Pb}$ & $\mathrm{Mg}$ & $\mathrm{Mn}$ \\
\hline \multirow{7}{*}{$\begin{array}{l}1632 a \\
1633 a \\
1634 a \\
1635 \\
1643 a \\
1645 \\
1646 \\
1648\end{array}$} & . & \multirow{3}{*}{$\begin{array}{l}1.11 \\
9.40\end{array}$} & 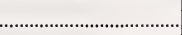 & \multirow[t]{2}{*}{12.4} & \multirow{2}{*}{$\begin{array}{l}(0.1) \\
.455\end{array}$} & \multirow{2}{*}{$\begin{array}{r}28 \\
(190)\end{array}$} \\
\hline & 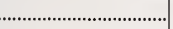 & & 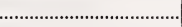 & & & \\
\hline & 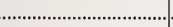 & & $\ldots$ & \multirow{4}{*}{$\begin{array}{c}1.9 \\
27 \\
714\end{array}$} & & \\
\hline & . & \multirow{3}{*}{$\begin{array}{l}0.239 \\
88 \\
11.3\end{array}$} & 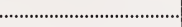 & & $\ldots$ & \multirow{3}{*}{$\begin{array}{l}21.4 \\
31 \\
785\end{array}$} \\
\hline & . & & . & & 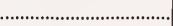 & \\
\hline & . & & (9) & & 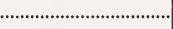 & \\
\hline & (20) & 3.91 & (42) & 0.655 & (.8) & $(860)$ \\
\hline
\end{tabular}




\begin{tabular}{|c|c|c|c|c|c|c|}
\hline SRM & $\mathrm{Hg}$ & Mo & $\mathrm{Ni}$ & $\mathrm{K}$ & $\mathrm{Rb}$ & Sm \\
\hline \multirow{7}{*}{$\begin{array}{l}1632 a \\
1633 a \\
1634 a \\
1635 \\
1643 a \\
1645 \\
1646 \\
1648\end{array}$} & 0.13 & & \multirow{2}{*}{$\begin{array}{c}19.4 \\
127\end{array}$} & \multirow{2}{*}{$\begin{array}{l}0.42 \\
1.88\end{array}$} & \multirow{2}{*}{$\begin{array}{c}(31) \\
72.4\end{array}$} & \\
\hline & .16 & (29) & & & & $\ldots$ \\
\hline & & ..... & \multirow{4}{*}{$\begin{array}{l}1.74 \\
55 \\
45.8\end{array}$} & & & . \\
\hline & \multirow{3}{*}{$\begin{array}{l}(.2) \\
1.1 \\
\ldots \ldots \ldots \ldots\end{array}$} & 95 & & & . & 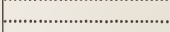 \\
\hline & & (1) & & (1.2) & $\ldots$ & 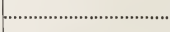 \\
\hline & & . & & & & \\
\hline & . & (1) & 82 & $(\mathbf{1 . 0 )}$ & 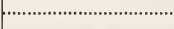 & $(4.4)$ \\
\hline
\end{tabular}

\begin{tabular}{|c|c|c|c|c|c|c|}
\hline SRM & $\mathrm{Sc}$ & $\mathrm{Se}$ & $\mathrm{Si}$ & $\mathrm{Ag}$ & $\mathrm{Na}$ & $\mathrm{Sr}$ \\
\hline \multirow{7}{*}{$\begin{array}{l}1632 a \\
1633 a \\
1634 a \\
1635 \\
1643 a \\
1645 \\
1646 \\
1648\end{array}$} & \multirow{2}{*}{$\begin{array}{l}(6.3) \\
(40)\end{array}$} & 2.6 & .. & . & \multirow{2}{*}{$\begin{array}{l}840 \\
0.17\end{array}$} & \multirow[b]{2}{*}{830} \\
\hline & & 10.3 & \multirow[t]{2}{*}{22.8} & $\ldots$ & & \\
\hline & \multirow{2}{*}{$(0.63)$} & \multirow{3}{*}{$\begin{array}{c}0.9 \\
11\end{array}$} & & 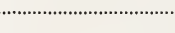 & \multirow{2}{*}{$(0.24)$} & \multirow{3}{*}{239} \\
\hline & & & 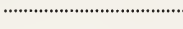 & 28 & & \\
\hline & \multirow[t]{2}{*}{ (2) } & & . & 2.8 & \multirow{2}{*}{ (.55) } & \\
\hline & & & & & & \\
\hline & (7) & (24) & 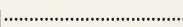 & (6) & $(.40)$ & \\
\hline
\end{tabular}

\begin{tabular}{|c|c|c|c|c|c|c|c|c|}
\hline SRM & $\mathrm{s}$ & $\mathrm{Tl}$ & Th & $\mathrm{Ti}$ & w & $\mathbf{U}$ & V & $\mathrm{Zn}$ \\
\hline \multirow{7}{*}{$\begin{array}{l}1632 a \\
1633 a \\
1634 a \\
1635 \\
1643 a \\
1645 \\
1646 \\
1648\end{array}$} & 1.62 & \multirow[b]{2}{*}{5.7} & \multirow{2}{*}{$\begin{array}{r}4.5 \\
24.7\end{array}$} & \multirow{2}{*}{$\begin{array}{c}(0.18) \\
(.8)\end{array}$} & ……………... & \multirow{2}{*}{$\begin{array}{l}1.28 \\
10.2\end{array}$} & \multirow{2}{*}{$\begin{array}{r}44 \\
(300)\end{array}$} & \multirow{2}{*}{$\begin{array}{r}28 \\
220\end{array}$} \\
\hline & 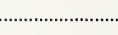 & & & & ... & & & \\
\hline & 0.33 & ………...... & 0.62 & $(.02)$ & …………........ & 0.24 & \multirow{3}{*}{$\begin{array}{c}5.2 \\
53 \\
23.5\end{array}$} & \multirow{3}{*}{$\begin{array}{r}4.7 \\
72 \\
1720\end{array}$} \\
\hline & & & & & & & & \\
\hline & .......................... & 1.44 & 1.62 & ............. & . & 1.11 & & \\
\hline & $\ldots$ & & & & & & & \\
\hline & $\ldots+\ldots \ldots \ldots \ldots$ & 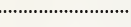 & (7.4) & (.40) & $(4.8)$ & $\ldots$ & (130) & 4760 \\
\hline
\end{tabular}

Organic Constituents

\begin{tabular}{c|c|c}
\hline SRM & \multicolumn{1}{|c|}{ Type } & \multicolumn{1}{c}{ Unit of Issue } \\
\hline 1580 & Shale Oil................................... & $\begin{array}{l}\text { Set of } 5-2 \mathrm{~mL} \text { ampoules. } \\
\text { Set of } 3 \text { columns. }\end{array}$ \\
\hline
\end{tabular}

\begin{tabular}{|c|c|c|}
\hline Constituents & $\begin{array}{c}\text { SRM } \\
1580 \\
(\mu \mathrm{g} / \\
\mathrm{g})\end{array}$ & SRM 1644 \\
\hline $\begin{array}{l}\text { Anthracene } \\
\text { Benzo [a] anthracene } \\
\text { Benzo [a] pyrene. } \\
\text { Benzo [e] pyrene } \\
\text { Fluoranthrene }\end{array}$ & $\begin{array}{r}21 \\
18 \\
54 \\
385 \\
407\end{array}$ & $\begin{array}{l}\text { (IN PREP). } \\
\text { (IN PREP). } \\
\text { (IN PREP). }\end{array}$ \\
\hline
\end{tabular}

\section{Permeation Tubes}

These SRM's are intended for calibrating air pollution monitoring apparatus, and may be used to verify air pollution analytical methods and procedures. Each tube is individually certified. 
Sulfur dioxide permeation tubes are available in three lengths $-2,5$, and 10 centimeters. The permeation rates are certified over the temperature range of 20 to $30^{\circ} \mathrm{C}$. The following table is provided as a guide in the selection of the appropriate length. The values in the table do not represent certified values for any SRM. The concentrations of $\mathrm{SO}_{2}$ in $\mathrm{ppm}$ are based on an approximate permeation rate of 0.28 micrograms per centimeter per minute at $25^{\circ} \mathrm{C}$, for flow rates of 1,5 , and 10 liters per minute.

\begin{tabular}{|c|c|c|c|c|c|c|}
\hline \multirow[t]{2}{*}{ SRM } & \multirow[t]{2}{*}{ Type } & \multirow[t]{2}{*}{$\begin{array}{l}\text { Tube } \\
\text { Length } \\
\text { (cm) }\end{array}$} & \multirow{2}{*}{$\begin{array}{c}\text { Perme- } \\
\text { ation } \\
\text { Rate } \\
(\mu \mathrm{g} / \mathrm{min})\end{array}$} & \multicolumn{3}{|c|}{$\begin{array}{c}\text { Typical } \\
\text { Concentrations (ppm) } \\
\text { Flow Rates (liters per } \\
\text { minute) }\end{array}$} \\
\hline & & & & (1) & (5) & $(10)$ \\
\hline 1625 & Sulfur Dioxide Permeation Tube & 10 & 2.8 & 1.07 & 0.214 & 0.107 \\
\hline 1626 & Sulfur Dioxide Permeation Tube & 5 & 1.4 & 0.535 & .107 & .0535 \\
\hline 1627 & Sulfur Dioxide Permeation Tube & 2 & 0.56 & .214 & .0428 & .0214 \\
\hline
\end{tabular}

\section{Nitrogen Dioxide}

Nitrogen dioxide permeation device (SRM 1629a) is calibrated at $25.0^{\circ} \mathrm{C}$ only. The temperature coefficient given with each tube provides the means to calculate permeation rates at other temperatures near $25^{\circ} \mathrm{C}$. The permeation rates for these tubes are between 0.5 and $1.5 \mu \mathrm{g} / \mathrm{min}$ at $25^{\circ} \mathrm{C}$. A tube with a rate of $1.0 \mu \mathrm{g} / \mathrm{min}$, in an air-flow of one liter per minute at $25^{\circ} \mathrm{C}$, will produce a concentration of $0.5 \mathrm{ppm}$ of $\mathrm{NO}_{2}$.

\section{Industrial Hygiene Standards}

\section{Freeze-Dried Urine}

These SRM's consist of two bottles of freeze-dried human urine, one containing a low and one an elevated level of the element certified.

\begin{tabular}{l|l|c|c}
\hline \multicolumn{1}{c|}{ SRM } & Element & $\begin{array}{c}\text { Low Level (mg/ } \\
\text { L) }\end{array}$ & $\begin{array}{c}\text { Elevated Level } \\
(\mathrm{mg} / \mathrm{L})\end{array}$ \\
\hline 2671 & Florine (F-) (IN PREP) & \\
\hline 2672 & Mercury (IN PREP) &
\end{tabular}

\section{Materials on Filter Media}

These SRM's consist of potentially hazardous materials deposited on filters to be used to determine the levels of these materials in industrial atmosphere.

\begin{tabular}{|c|c|c|c|c|c|c|}
\hline \multirow{2}{*}{ SRM } & \multirow{2}{*}{ Type } & \multirow{2}{*}{ Material Certified } & \multicolumn{4}{|c|}{ Quantity Certified ( $\mu \mathrm{g} /$ filter $)$} \\
\hline & & & I & II & III & IV \\
\hline 2674 & Lead on Filter Media ........................................... & 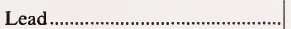 & 100 & 303 & 1505 & 1.4 \\
\hline \multirow[t]{2}{*}{2673} & Sulfate and Nitrate on Filter Media...................... & 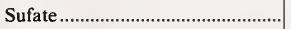 & 500 & 2000 & 7000 & \\
\hline & & Nitrate............................................... & 100 & 1000 & 2500 & \\
\hline 2675 & 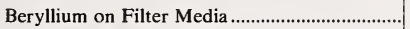 & Beryllium......................................... & 0.052 & 0.26 & 1.00 & \\
\hline \multirow[t]{4}{*}{$2676 \mathrm{a}$} & 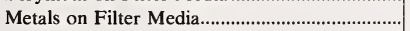 & 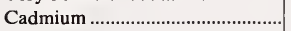 & 1.02 & 2.50 & 10.18 & \\
\hline & & Lead & 6.96 & 15.23 & 29.64 & ..................... \\
\hline & & Manganese ....................................... & 1.97 & 9.89 & 19.70 & 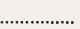 \\
\hline & & 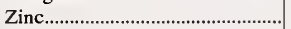 & 9.86 & 49.52 & 99.22 & ...................... \\
\hline \multirow[t]{2}{*}{2679} & Quartz on Filter Media & 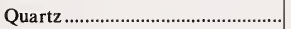 & 3.8 & 29.9 & 76.1 & 193.2 \\
\hline & & 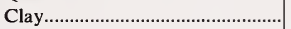 & $(400)$ & $(370)$ & $(320)$ & $(200)$ \\
\hline
\end{tabular}


These SRM's are intented for use in the calibration of apparatus and the evaluation of methods used in the analysis of materials of interest to law enforcement agencies. (For details on SRM 1820, see Refractive Index Standards, page 77.)

\begin{tabular}{|c|c|c|c|}
\hline SRM & Type & Certification & Unit of Issue \\
\hline 1820 & Glass, Borosilicate & 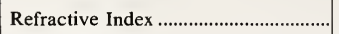 & 2 slabs; one polished, one unpolished. \\
\hline 1822 & Glass, Window (Soda-Lime) ............................ & Refractive Index ................................... & 2 slabs; one polished, one unpolished. \\
\hline 1823 & Silicone liquids-I \& II & Refractive Index .................................. & Set of $2 ; 60 \mathrm{~mL}$ each. \\
\hline
\end{tabular}

\section{Metallo-Organic Compounds}

These SRM's are intended for the preparation of solutions in oils of known and reproducible concentrations of metals. Because "matrix" effects occur, it is desirable to prepare the standard solutions in oil identical or similar to the oil being studied. Possession of an adequate collection of these metallo-organic SRM's permits the preparation of any desired blend of known concentrations of metal in the appropriate lubricating oil. They are used primarily for the calibration of spectrochemical equipment used in the determination of metals in lubricating oil. This technique is used extensively in the defense program, the transportation industry, and other industries where the consequences of failure of a moving metal part may range from inconvenient to catastrophic.

The Certificate supplied with each SRM gives the percentage of the element of interest and directions for preparing a solution of known concentration in lubricating oil.

\begin{tabular}{|c|c|c|c|c|}
\hline \multirow[b]{2}{*}{ SRM } & \multicolumn{2}{|l|}{ Constituent Certified } & \multirow{2}{*}{$\begin{array}{c}\text { Wt/ } \\
\text { Unit } \\
\text { (grams) }\end{array}$} & \multirow[b]{2}{*}{ Type } \\
\hline & Element & $\begin{array}{c}\text { (wt. } \\
\text { percent) }\end{array}$ & & \\
\hline 1075a & Al & 8.07 & 5 & Aluminum 2-ethylhexanoate. \\
\hline $1051 \mathrm{~b}$ & Ва & 28.7 & 5 & Barium cyclohexanebutyrate. \\
\hline $1053 a$ & 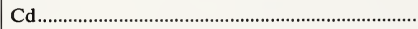 & 24.8 & 5 & Cadmium cyclohexanebutyrate. \\
\hline 1074a & $\mathrm{Ca}$ & 12.5 & 5 & Calcium 2-ethylhexanoate. \\
\hline $1078 \mathrm{~b}$ & ( & 9.6 & 5 . & Tris(1-phenyl-1,3-butanediono)chromium (III). \\
\hline $1055 \mathrm{~b}$ & Со & 14.8 & 5 & Cobalt cyclohexanebutyrate. \\
\hline $1080 \mathrm{a}$ & $\mathrm{Cu}$ & 16.37 & 5 & Bis (1-phenyl-1,3-butanediono) copper (II). \\
\hline $1079 b$ & $\mathrm{Fe}$ & 10.45 & 5 & Tris (1-phenyl-1,3-butanediono) iron (III). \\
\hline $1060 \mathrm{a}$ & $\mathrm{Li}$ & 4.1 & 5 & Lithium cyclohexanebutyrate. \\
\hline $1061 \mathrm{c}$ & $\mathrm{Mg}$ & 6.45 & 5 & Magnesium cyclohexanebutyrate. \\
\hline $1062 \mathrm{~b}$ & $\mathrm{Mn}$ & 13.2 & 5 & Manganous cyclohexanebutyrate. \\
\hline $1065 b$ & $\mathrm{Ni}$ & 13.89 & 5 & Nickel cyclohexanebutyrate. \\
\hline $1071 \mathrm{~b}$ & 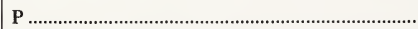 & 9.48 & 5 & Triphenyl phosphate. \\
\hline $1066 \mathrm{a}$ & $\mathrm{Si}$ & 14.14 & 5 & Octaphenylcyclotetrasiloxane. \\
\hline $1077 \mathrm{a}$ & 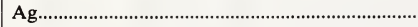 & 42.60 & 5 & Silver 2-ethylhexanoate. \\
\hline $1069 \mathrm{~b}$ & $\mathrm{Na}$ & 12.0 & 5 & Sodium cyclohexanebutyrate. \\
\hline 1070a & $\mathrm{Sr}$ & 20.7 & 5 & Strontium cyclohexanebutyrate. \\
\hline $1057 \mathrm{~b}$ & Sn & 22.95 & 5 & Dibutyltin bis (2-ethylhexanoate). \\
\hline $1052 \mathrm{~b}$ & V & 13.01 & 5 & Bis (1-phenyl-1,3-butanediono) oxovanadium (IV). \\
\hline $1073 \mathrm{~b}$ & Zn & 16.66 & 5 & Zinc cyclohexanebutyrate. \\
\hline
\end{tabular}


These SRM's consist of 12 metallo-organic compounds blended into a base oil.

\begin{tabular}{|c|c|c|c|c|c|c|c|c|c|}
\hline \multirow{2}{*}{ SRM } & \multirow{2}{*}{\multicolumn{5}{|c|}{ Type }} & \multirow{2}{*}{\multicolumn{2}{|c|}{ Unit }} & \multicolumn{2}{|c|}{ Elements, $\mu \mathrm{g} / \mathrm{g}$} \\
\hline & & & & & & & & $\mathrm{Ag}$ & $\mathrm{Al}$ \\
\hline $\begin{array}{l}1084 \\
1085\end{array}$ & \multicolumn{5}{|c|}{$\begin{array}{l}\text { Wear metal-in-oil }(100 \mathrm{ppm}) \\
\text { Wear metal-in-oil }(300 \mathrm{ppm})\end{array}$} & \multicolumn{2}{|c|}{$85 \mathrm{~mL} . \ldots \ldots \ldots \ldots \ldots$} & $\begin{array}{l}(\quad) \\
()\end{array}$ & $\begin{array}{r}(98.6) \\
(297)\end{array}$ \\
\hline $\mathrm{Cr}$ & $\mathrm{Cu}$ & $\mathrm{Fe}$ & $\mathrm{Mg}$ & Mo & $\mathrm{Ni}$ & $\mathrm{Pb}$ & $\mathrm{Si}$ & $\mathrm{Sn}$ & $\mathrm{Ti}$ \\
\hline $\begin{array}{l}(102) \\
(298)\end{array}$ & $\begin{array}{r}(97.8) \\
(299)\end{array}$ & $\begin{array}{l}(101) \\
(302)\end{array}$ & $\begin{array}{l}(100) \\
(297)\end{array}$ & $\left.\begin{array}{ll}( \\
(\end{array}\right)$ & $\begin{array}{r}(98.9) \\
(301)\end{array}$ & $\begin{array}{l}(102) \\
(299)\end{array}$ & $\begin{array}{l}() \\
()\end{array}$ & $\begin{array}{l}() \\
()\end{array}$ & $\begin{array}{r}(102) \\
()\end{array}$ \\
\hline
\end{tabular}

\section{Fertilizer Standards}

These SRM's are intended for use in the fertilizer industry as working standards for the determination of the certified constituents.

\begin{tabular}{|c|c|c|c|c|c|}
\hline \multirow[t]{2}{*}{ SRM } & \multirow{2}{*}{ Type } & \multirow{2}{*}{$\begin{array}{l}\text { Wt/Unit } \\
\text { (grams) }\end{array}$} & \multicolumn{3}{|c|}{$\begin{array}{c}\text { Certified Composition (Wt } \\
\text { percent) }\end{array}$} \\
\hline & & & $\mathrm{N}$ & $\mathbf{P}$ & $\mathrm{K}$ \\
\hline 193 & Potassium Nitrate & \multirow{3}{*}{$\begin{array}{l}90 \\
90 \\
90\end{array}$} & \multirow{3}{*}{$\begin{array}{l}13.85 \\
12.15\end{array}$} & \multirow{3}{*}{$\begin{array}{l}29.92 \\
22.74\end{array}$} & \multirow[t]{2}{*}{38.66} \\
\hline 194 & 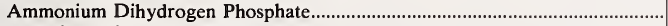 & & & & \\
\hline 200 & Potassium Dihydrogen Phosphate & & & & 28.76 \\
\hline
\end{tabular}

\section{Ores}

These SRM's are intended for use in checking the accuracy of assay methods. They are certified for their content of elements of economic interest, and occasionally, have additional data given for information only. These SRM's are supplied in the form of fine powders, usually less than $0.15 \mathrm{~mm}$.

\begin{tabular}{|c|c|c|c|c|c|c|c|}
\hline \multirow{2}{*}{ SRM } & \multirow{2}{*}{ Type } & \multirow{2}{*}{$\begin{array}{c}\text { Wt/ } \\
\text { Unit } \\
\text { (grams) }\end{array}$} & \multicolumn{5}{|c|}{ Chemical Compositions (Nominal Weight Percent) } \\
\hline & & & $\mathrm{CaF}_{2}$ & $\mathrm{Li}_{2} \mathrm{O}$ & $\mathrm{Cu}$ & $\operatorname{Re}$ & Mo \\
\hline $79 a$ & Fluorspar & 120 & 97.39 & & & & \\
\hline 180 & Fluospar, high grade & 120 & 98.8 & & & & \\
\hline 181 & Lithium (Spodumene) & 45 & ............. & 6.4 & & & \\
\hline 182 & Lithium (Petalite) & 45 & 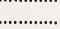 & 4.3 & & & \\
\hline 183 & 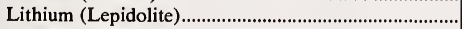 & 45 & & 4.1 & & & \\
\hline 330 & Copper, millheads & 100 & & & 0.84 & $0.3 \mathrm{ppm}$ & 0.018 \\
\hline 331 & 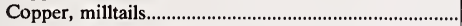 & 100 & & & .091 & 0.4 ppm & .0022 \\
\hline 332 & Copper, concentrate & 50 & & ................ & 28.45 & $10.2 \mathrm{ppm}$ & .64 \\
\hline 333 & Molybdenum, concentrate & 55 & & .................. & 1.038 & 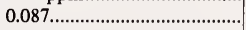 & 55.3 \\
\hline
\end{tabular}




\begin{tabular}{|c|c|c|c|c|c|c|c|c|}
\hline \multirow{2}{*}{ SRM } & \multirow{2}{*}{ Type } & \multirow{2}{*}{$\begin{array}{l}\text { Wt/Unit } \\
\text { (grams) }\end{array}$} & \multicolumn{6}{|c|}{ Chemical Composition (Nominal Weight Percent) } \\
\hline & & & $\mathrm{Al}_{2} \mathrm{O}_{3}$ & $\mathrm{BaO}$ & $\mathrm{Cd}$ & $\mathrm{CdO}$ & $\mathrm{CaO}$ & $\mathrm{CO}_{2}$ \\
\hline $27 \mathrm{f}$ & 1ron Ore, Sibley & 100 & 0.82 & & & & 0.039 & \\
\hline 690 & Iron Ore, Canada (Conc.) & 150 & .18 & …….... & & .............. & .20 & ............ \\
\hline 691 & 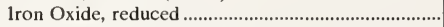 & IN PREP & $\left({ }^{*}\right)$ & & & & $\left({ }^{*}\right)$ & ........ \\
\hline 692 & Iron Ore, Labrador & 150 & 1.41 & & & & .023 & ......... \\
\hline 693 & Iron Ore, Nimba & 150 & 1.02 & & & & .016 & -............ \\
\hline $69 \mathrm{~b}$ & 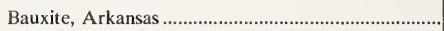 & 80 & 49.3 & $<0.01$ & & & .12 & \\
\hline 696 & Bauxit & 80 & 54.5 & $(0.004)$ & & & .018 & $\ldots$ \\
\hline 697 & Bauxite, Dominican & 80 & 45.8 & $(.015)$ & & & .71 & ........ \\
\hline 698 & Bauxite, Jamaican & 80 & 48.2 & $(.008)$ & & & .62 & ............ \\
\hline $120 \mathrm{~b}$ & Phosphate Rock, Florida & 90 & 1.06 & & & 0.002 & 49.40 & 2.79 \\
\hline 277 & Tungsten Concentrate & 100 & ……………....... & Ta $(0.20)$ & & & $\mathrm{Ca}(0.37)$ & \\
\hline $113 \mathrm{a}$ & 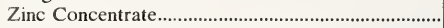 & 100 & 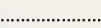 & - la & 0.78 & & 1.1, & ............. \\
\hline 329 & Zinc Concentrate & 100 & ............ & ........................ & .14 & ................. & 0.08 & ............. \\
\hline
\end{tabular}

\begin{tabular}{|c|c|c|c|c|c|c|c|c|c|}
\hline SRM & $\mathrm{Cu}$ & $\mathrm{Cr}_{2} \mathrm{O}_{3}$ & F & In & Total Fe & $\mathrm{Fe}_{2} \mathrm{O}_{3}$ & $\mathrm{~Pb}$ & $\mathrm{MgO}$ & $\mathrm{MnO}$ \\
\hline $27 \mathrm{f}$ & & & & & 65.97 & & & 0.019 & 0.011 \\
\hline 690 & & & & & 66.85 & & & .18 & .23 \\
\hline 691 & & & & & $(*)$ & 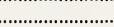 & ………… & $\left(^{*}\right)$ & $(*)$ \\
\hline 692 & & & & ․․․․․․………. & 59.58 & ․․․․․․․․․…… & 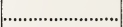 & .035 & .46 \\
\hline 693 & & & 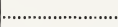 & 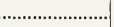 & 65.11 & 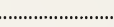 & 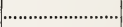 & .013 & .091 \\
\hline $69 \mathrm{~b}$ & 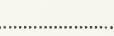 & 0.011 & & & . & 7.14 & ㄱ..и…… & .085 & .09 \\
\hline 696 & . & .047 & 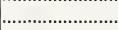 & 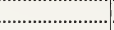 & (1) & 8.70 & 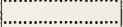 & .012 & .004 \\
\hline 697 & 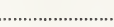 & .100 & ․․․․………....... & ․․․․․․……... & ․․․․……….... & 20.0 & 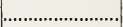 & .18 & .41 \\
\hline 698 & ․․…………….... & .080 & ……………...... & ……………...... & ․․․………........ & 19.6 & 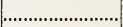 & .058 & .38 \\
\hline $120 \mathrm{~b}$ & & & 3.84 & & & 1.10 & & .28 & .032 \\
\hline 277 & & $\mathrm{Nb}(1.00)$ & & & (7.4) & 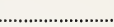 & $(0.07)$ & & $\mathrm{Mn}(10.0)$ \\
\hline $113 a$ & 031 & & 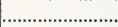 & ND & 2.08 & 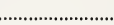 & 2.80 & .75 & ․․……… \\
\hline 329 & $.13_{2}$ & 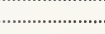 & 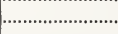 & 0.019 & $12.9_{4}$ & 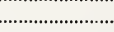 & $6.0_{6}$ & $.16_{5}$ & . \\
\hline
\end{tabular}

*To be certified.

\begin{tabular}{|c|c|c|c|c|c|c|c|c|c|}
\hline SRM & $\mathbf{P}$ & $\mathrm{P}_{2} \mathrm{O}_{5}$ & $\mathrm{~K}_{2} \mathrm{O}$ & $\mathrm{SiO}_{2}$ & $\mathrm{Ag}$ & $\mathrm{Na}_{2} \mathrm{O}$ & S & $\mathrm{SO}_{3}$ & $\mathrm{TiO}_{2}$ \\
\hline $27 \mathrm{f}$ & 0.041 & … & 0.008 & 4.17 & $\ldots \ldots \ldots$ & 0.012 & 0.005 & & 0.019 \\
\hline 690 & .011 & ........................ & .0030 & 3.71 & ........................ & .003 & .003 & ........ & .022 \\
\hline 691 & & 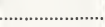 & $\left({ }^{*}\right)$ & $\left({ }^{*}\right)$ & …………........... & & & 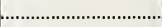 & $(*)$ \\
\hline 692 & .039 & .......................... & .039 & 10.14 & …………............ & .008 & .005 & 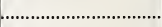 & .045 \\
\hline 693 & .056 & 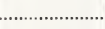 & .0028 & 3.87 & ............................. & .0028 & .005 & ............. & .035 \\
\hline $69 \mathrm{~b}$ & & 0.012 & .80 & 13.4 & ……….... & .030 & & 0.63 & 2.0 \\
\hline 696 & .................... & .050 & .009 & 3.79 & $\ldots$ & $(.007)$ & ....... & .21 & 2.64 \\
\hline 697 & .................... & .97 & .062 & 6.81 & ……………......... & $(.036)$ & ................... & .13 & 2.52 \\
\hline 698 & ..................... & .37 & .010 & 0.69 & ...................... & $(.015)$ & .................... & .22 & 2.38 \\
\hline $120 \mathrm{~b}$ & & 34.57 & .12 & 4.68 & & .35 & & & 0.15 \\
\hline 277 & $(.03)$ & & & $\mathrm{Si}(0.85)$ & & 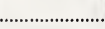 & $(.25)$ & $\mathrm{O}_{2}(21.4)$ & $\mathrm{Ti}(2.2)$ \\
\hline $113 a$ & & & & (1.54) & $0.046_{7}$ & & (30.6) & & \\
\hline 329 & & & .............. & $(0.61)$ & .0089 & ............................ & (31.7) & 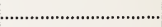 & ......................... \\
\hline
\end{tabular}




\begin{tabular}{|c|c|c|c|c|c|c|c|c|}
\hline SRM & $\mathbf{U}$ & $\mathrm{V}_{2} \mathrm{O}_{5}$ & $\mathrm{WO}_{3}$ & $\mathrm{Zn}$ & $\mathrm{ZnO}$ & $\mathrm{ZrO}_{2}$ & $\begin{array}{l}\text { Loss on } \\
\text { ignition }\end{array}$ & Moisture \\
\hline \multicolumn{9}{|l|}{$27 f$} \\
\hline 690 & & & & & & & & \\
\hline 691 & ........................ & 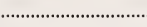 & (1)................... & $\ldots$ & 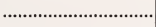 & ............................ & $\ldots \ldots \ldots \ldots \ldots$ & 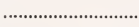 \\
\hline 692 & & & & & s & & & 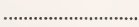 \\
\hline 693 & ................ & & $\ldots$. & & & ................... & ................... & 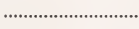 \\
\hline $69 b$ & & 0.03 & & & 0.003 & & 27.22 & \\
\hline 696 & .......................... & .063 & & 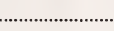 & .0014 & 0.14 & 29.9 & 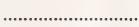 \\
\hline 697 & $\ldots \ldots \ldots \ldots \ldots$ & .072 & 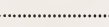 & 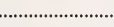 & .037 & .065 & 22.1 & 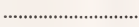 \\
\hline 698 & & .064 & ........... & 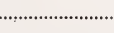 & .029 & .061 & 27.3 & 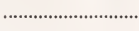 \\
\hline $120 \mathrm{~b}$ & $128.4 \mu \mathrm{g} / \mathrm{g}$ & & & & & & & \\
\hline 277 & $\ldots \ldots \ldots \ldots$ & Mo (0.06) & 67.4 & 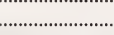 & Sn $(0.54)$ & ……................... & ............................. & . \\
\hline $113 a$ & & & & 57.3 & & & 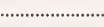 & 0.08 \\
\hline 329 & .......................... & 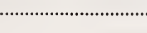 & $\ldots$ & 45.5 & 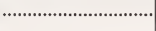 & ........................ & 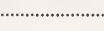 & .45 \\
\hline
\end{tabular}

Minerals, Refractories, Glasses, and Carbides

These SRM's are supplied in the form of powders, usually less than $0.15 \mathrm{~mm}$. They are intended to provide materials for checking the accuracy of methods used in the analysis of similar materials, primarily in the glass, ceramics, and steel industries.

Minerals

\begin{tabular}{|c|c|c|c|c|c|c|c|c|}
\hline \multirow{2}{*}{ SRM } & \multirow{2}{*}{ Type } & \multirow{2}{*}{$\begin{array}{c}\text { Wt/ } \\
\text { Unit } \\
\text { (grams) }\end{array}$} & \multicolumn{6}{|c|}{$\begin{array}{l}\text { Chemical Composition (Normal Weight } \\
\text { Percent as the Oxide) }\end{array}$} \\
\hline & & & $\mathrm{SiO}_{2}$ & $\mathrm{Fe}_{2} \mathrm{O}_{3}$ & $\mathrm{Al}_{2} \mathrm{O}_{3}$ & $\mathrm{TiO}_{2}$ & $\mathrm{MnO}$ & $\mathrm{CaO}$ \\
\hline lc & Limestone, argillaceous ${ }^{*}$ & 50 & 6.84 & 0.55 & 1.30 & 0.07 & 0.025 & 50.3 \\
\hline $88 \mathbf{a}$ & Limestone, dolomitic & 50 & 1.20 & .28 & 0.19 & .02 & .03 & 30.1 \\
\hline $70 a$ & Feldspar, potash & 40 & 67.1 & .075 & 17.9 & .01 & & 0.11 \\
\hline $99 a$ & Feldspar, soda & 40 & 65.2 & .065 & 20.5 & .007 & & 2.14 \\
\hline $97 \mathbf{a}$ & Clay, flint & 60 & 43.7 & .45 & 38.8 & 1.90 & & 0.11 \\
\hline $98 \mathrm{a}$ & Clay, plastic & 60 & 48.9 & 1.34 & 33.2 & 1.61 & & .31 \\
\hline $81 \mathrm{a}$ & Glass sand & 75 & ............... & 0.082 & 0.66 & 0.12 & & \\
\hline $165 a$ & Glass sand (low iron) & 75 & & .012 & .059 & .011 & & \\
\hline $154 \mathrm{~b}$ & Titanium dioxide & 90 & & - & & 99.74 & & \\
\hline
\end{tabular}

*Information values only: $\mathrm{S}(0.1) ; \mathrm{SO}_{3}(0.1) ; \mathrm{F}(0.009)$; and $\mathrm{Cl}(0.004)$.

\begin{tabular}{|c|c|c|c|c|c|c|c|c|c|c|c|c|}
\hline SRM & $\mathrm{SrO}$ & $\mathrm{MgO}$ & $\mathrm{Cr}_{2} \mathrm{O}_{3}$ & $\mathrm{Na}_{2} \mathrm{O}$ & $\mathrm{K}_{2} \mathrm{O}$ & $\mathrm{Li}_{2} \mathrm{O}$ & $\mathrm{ZrO}_{2}$ & $\mathrm{BaO}$ & $\mathrm{Rb}_{2} \mathrm{O}$ & $\mathrm{P}_{2} \mathrm{O} 5$ & $\mathrm{CO}_{2}$ & $\begin{array}{l}\text { Loss } \\
\text { on } \\
\text { Igni- } \\
\text { tion }\end{array}$ \\
\hline 1c & 0.030 & 0.42 & ..................... & 0.02 & 0.28 & & & & & 0.04 & & 39.9 \\
\hline $88 a$ & .010 & 21.3 & ................. & .01 & .12 & & & $\ldots \ldots$. & ............ & .01 & 46.6 & 46.7 \\
\hline $70 a$ & 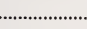 & 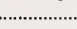 & .................... & 2.55 & 11.8 & & & 0.02 & 0.06 & & & 0.40 \\
\hline $99 a$ & ..................... & 0.02 & ................... & 6.2 & 5.2 & 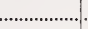 & ............. & .26 & ..................... & .02 & & .26 \\
\hline $97 a$ & .18 & .15 & 0.03 & 0.037 & 0.50 & 0.11 & 0.063 & .078 & .................... & .36 & ................... & 13.32 \\
\hline $98 a$ & .039 & .42 & .03 & .082 & 1.04 & .070 & .042 & .03 & & .11 & & 12.44 \\
\hline $81 \mathrm{a}$ & & & $46 \mu \mathrm{g} / \mathrm{g}$ & & & & .034 & & & & & ............... \\
\hline $165 a$ & ....... & & $(1.1 \mu / g)$ & & & & .006 & & & & & \\
\hline $154 b$ & 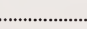 & & . & ........................ & 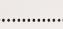 & ................ & & & $\ldots$ & & & ....... \\
\hline & & & & & & & & & & & & \\
\hline
\end{tabular}




\section{Refractories}

\begin{tabular}{|c|c|c|c|c|c|c|c|c|c|c|}
\hline \multirow[t]{2}{*}{ SRM } & \multirow[t]{2}{*}{ Type } & \multirow{2}{*}{$\underset{\underset{\text { (grams) }}{\text { Unit }}}{\mathrm{Wt}}$} & \multicolumn{8}{|c|}{$\begin{array}{c}\text { Chemical Composition (Nominal Weight Percent as the } \\
\text { Oxide) }\end{array}$} \\
\hline & & & $\mathrm{SiO}_{2}$ & $\mathrm{Al}_{2} \mathrm{O}_{3}$ & $\mathrm{Fe}_{2} \mathrm{O}_{3}$ & $\mathrm{FeO}$ & $\mathrm{TiO}_{2}$ & $\mathrm{ZrO}_{2}$ & $\mathrm{MnO}$ & $\mathrm{P}_{2} \mathrm{O}_{5}$ \\
\hline 76a & Burnt Refractory $\left(\mathrm{Al}_{2} \mathrm{O}_{3}-40 \%\right)$ & 75 & 54.9 & 38.7 & 1.6 & & $2.0_{3}$ & $(0.15)$ & & 0.120 \\
\hline $77 a$ & Burnt Refractory $\left(\mathrm{Al}_{2} \mathrm{O}_{3}-60 \%\right) \ldots \ldots \ldots . .$. & 75 & 35.0 & 60.2 & $1.0_{0}$ & & $2.6_{6}$ & $(.21)$ & & .092 \\
\hline 78a & Burnt Refractory $\left(\mathrm{Al}_{2} \mathrm{O}_{3}-70 \%\right) \ldots \ldots \ldots \ldots \ldots \ldots \ldots \ldots \ldots \ldots \ldots \ldots \ldots$ & 75 & 19.4 & 71.7 & 1.2 & & $3.2_{2}$ & $(.31)$ & & 1.3 \\
\hline 103a & Chrome refractory & 60 & 4.6 & 29.96 & & 12.43 & 0.22 & .01 & 0.11 & 0.01 \\
\hline 198 & Silica refractory & 45 & & 0.16 & 0.66 & & .02 & $<.01$ & .008 & .022 \\
\hline 199 & Silica refractory & 45 & ........... & .48 & .74 & ............... & .06 & .01 & .007 & .015 \\
\hline
\end{tabular}

\begin{tabular}{|c|c|c|c|c|c|c|c|c|}
\hline SRM & $\mathrm{Cr}_{2} \mathrm{O}_{3}$ & $\mathrm{CaO}$ & $\mathrm{MgO}$ & $\mathrm{Li}_{2} \mathrm{O}$ & $\mathrm{Na}_{2} \mathrm{O}$ & $\mathrm{K}_{2} \mathrm{O}$ & SrO & $\begin{array}{l}\text { Loss on } \\
\text { Ignition }\end{array}$ \\
\hline $76 a$ & & 0.22 & 0.52 & 0.042 & 0.07 & 1.33 & 0.037 & $(0.34)$ \\
\hline $77 \mathrm{a}$ & 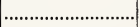 & .05 & .38 & $.02_{5}$ & .037 & 0.09 。 & .009 & $(.22)$ \\
\hline $78 a$ & 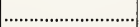 & .11 & .70 & .12 & .078 & 1.22 & .25 & (.42) \\
\hline $103 a$ & 32.06 & .69 & 18.54 & & ……...... & & $\ldots \ldots \ldots \ldots$ & ............... \\
\hline 198 & 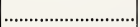 & 2.71 & 0.07 & .001 & .012 & 0.017 & 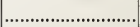 & .21 \\
\hline 199 & 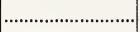 & 2.41 & .13 & .002 & .015 & .094 & 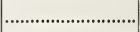 & .17 \\
\hline
\end{tabular}

Glasses

\begin{tabular}{|c|c|c|c|c|c|c|c|c|c|c|}
\hline \multirow{2}{*}{ SRM } & \multirow{2}{*}{ Type } & \multirow{2}{*}{ Unit Size } & \multicolumn{8}{|c|}{ Chemical Composition (Nominal Weight Percent) } \\
\hline & & & $\mathrm{SiO}_{2}$ & $\mathrm{PbO}$ & $\mathrm{Al}_{2} \mathrm{O}_{3}$ & $\mathrm{Fe}_{2} \mathrm{O}_{3}$ & $\mathrm{ZnO}$ & $\mathrm{MnO}$ & $\mathrm{TiO}_{2}$ & $\mathrm{ZrO}_{2}$ \\
\hline 89 & Lead-Barium.............................. & 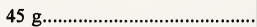 & 65.35 & 17.50 & 0.18 & 0.049 & & 0.088 & 0.01 & 0.005 \\
\hline 91 & 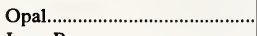 & $45 \mathrm{~g}$ & 67.53 & 0.097 & 6.01 & .081 & 0.08 & .008 & .019 & .0095 \\
\hline 92 & Low-Boron ............................... & 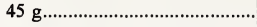 & & & & & & & & \\
\hline $93 a$ & High-Boron...................................... & Wafer $32 \mathrm{~mm} \mathrm{D} \times 6 \mathrm{~mm} \ldots \ldots . .$. & 80.8 & (n).............. & 2.3 & .029 & .......... & ................ & .012 & .03 \\
\hline \multirow[t]{2}{*}{620} & Soda-Lime, Flat ......................... & 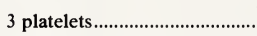 & & & & & & & & \\
\hline & 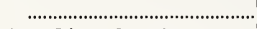 & $35 \times 35 \times 3 \mathrm{~mm}$ & 72.1 & & 1.8 & .04 & & ........... & .02 & ............ \\
\hline \multirow[t]{2}{*}{621} & Soda-Lime, Container............. & 3 disks & & & & & & & & \\
\hline & & $38 \mathrm{~mm} \mathrm{D} \times 5 \mathrm{~mm}$ & 71.14 & & 2.77 & .040 & & .......... & .014 & .009 \\
\hline 1830 & Soda-Lime Float ...................... & 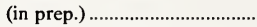 & & & & & & & & ................ \\
\hline 1831 & Soda-Lime Float ........................ & 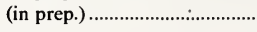 & & & & & & & & .......... \\
\hline
\end{tabular}

\begin{tabular}{|c|c|c|c|c|c|c|c|c|c|c|c|c|c|}
\hline SRM & $\mathrm{CaO}$ & $\mathrm{BaO}$ & $\mathrm{MgO}$ & $\mathrm{K}_{2} \mathrm{O}$ & $\mathrm{Na}_{2} \mathrm{O}$ & $\mathrm{B}_{2} \mathrm{O}_{3}$ & $\mathrm{P}_{2} \mathrm{O}_{5}$ & $\mathrm{As}_{2} \mathrm{O}_{5}$ & $\mathrm{As}_{2} \mathrm{O}_{3}$ & $\mathrm{SO}_{3}$ & $\mathrm{Cl}$ & F & $\begin{array}{c}\text { Loss } \\
\text { on } \\
\text { Igni- } \\
\text { tion }\end{array}$ \\
\hline 89 & 0.21 & 1.40 & 0.03 & 8.40 & 5.70 & & 0.23 & 0.36 & 0.03 & 0.03 & 0.05 & & 0.32 \\
\hline 91 & 10.48 & ...……......... & .008 & 3.25 & 8.48 & & .022 & .102 & .091 & ..………....... & .014 & 5.72 & .................... \\
\hline 92 & & & & & & 0.70 & & & & & & ................. & ..................... \\
\hline $93 a$ & $<0.02$ & ................... & $<.01$ & 0.01 & 4.0 & 12.6 & & & & & & & ...……......... \\
\hline 620 & 7.1 & 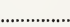 & 3.7 & .4 & 14.4 & $\ldots$ & ……….......... & ........... & .06 & .3 & ............ & ........... & ...……….... \\
\hline 621 & 10.71 & 0.12 & 0.27 & 2.01 & 12.71 & ............ & …………...... & ...………........ & .03 & .13 & ………........ & .................... & ………......... \\
\hline
\end{tabular}




\begin{tabular}{|c|c|c|c|}
\hline SRM & Type & $\begin{array}{c}\mathrm{Wt} / \\
\text { Unit } \\
\text { (grams) }\end{array}$ & $\begin{array}{l}\text { Total Carbon } \\
\text { (Wt. \%) }\end{array}$ \\
\hline $276 a$ & Tungsten Carbide & 75 & 6.11 \\
\hline
\end{tabular}

\section{Cements}

These SRM's are furnished for x-ray spectroscopic analysis and for chemical analysis of cements and related materials. Because these SRM's are hygroscopic, each unit consists of three sealed vials each containing approximately $5 \mathrm{~g}$ of material.

\begin{tabular}{|c|c|c|c|c|c|c|c|c|}
\hline \multirow{2}{*}{ SRM } & \multirow{2}{*}{ Type } & \multirow{2}{*}{$\begin{array}{l}\text { Wt/Unit } \\
\text { (grams) }\end{array}$} & \multicolumn{6}{|c|}{ Chemical Composition (Nominal Weight Percent) } \\
\hline & & & $\mathrm{CaO}$ & $\mathrm{SiO}_{2}$ & $\mathrm{Al}_{2} \mathrm{O}_{3}$ & $\mathrm{Fe}_{2} \mathrm{O}_{3}$ & $\mathrm{SO}_{3}$ & $\mathrm{MgO}$ \\
\hline 633 & Portland cement (red) & 15 & 64.50 & $21.8_{8}$ & $3.7_{8}$ & 4.20 & 2.20 & $1.0_{4}$ \\
\hline 634 & Portland cement (gold) & 15 & $62.5_{8}$ & $20.7_{3}$ & $5.2_{1}$ & 2.84 & $2.2_{1}$ & 3.3。 \\
\hline 635 & Portland cement (blue) & 15 & $59.8_{3}$ & $18.4_{1}$ & 6.2 & 2.61 & $7.0_{7}$ & $1.2_{3}$ \\
\hline 636 & 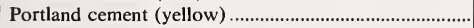 & 15 & $63.5_{4}$ & $23.2_{2}$ & $3.0_{2}$ & 1.61 & $2.3_{1}$ & $3.9_{5}$ \\
\hline 637 & 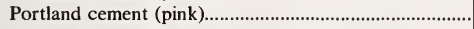 & 15 & $66.0_{4}$ & $23.0_{7}$ & $3.2 \mathrm{~s}$ & 1.80 & $2.3_{8}$ & 0.6 \\
\hline 638 & Portland cement (green) & 15 & 62.09 & $21.4 \mathrm{~s}$ & $4.4_{5}$ & 3.55 & $2.3_{4}$ & $3.8_{3}$ \\
\hline 639 & 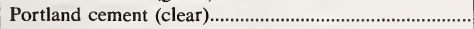 & 15 & $65.7_{6}$ & $21.6_{1}$ & $4.2_{8}$ & 2.40 & $2.4_{8}$ & $1.2_{6}$ \\
\hline
\end{tabular}

\begin{tabular}{|c|c|c|c|c|c|c|c|c|c|c|}
\hline SRM & $\mathrm{K}_{2} \mathrm{O}$ & $\mathrm{TiO}_{2}$ & $\mathrm{Na}_{2} \mathrm{O}$ & $\mathrm{SrO}$ & $\mathrm{P}_{2} \mathrm{O}_{5}$ & $\mathrm{Mn}_{2} \mathrm{O}_{3}$ & $\mathrm{~F}$ & $\mathrm{ZnO}$ & $\mathrm{Cr}_{2} \mathrm{O}_{3}$ & $\begin{array}{l}\text { Loss on } \\
\text { Ignition }\end{array}$ \\
\hline 633 & 0.17 & 0.24 & 0.64 & 0.31 & 0.24 & 0.04 & 0.08 & 0.01 & 0.01 & $0.7_{\mathrm{s}}$ \\
\hline 634 & .42 & .29 & .15 & .12 & .10 & .28 & .08 & .02 & .08 & $1.6_{2}$ \\
\hline 635 & .45 & .32 & .07 & .21 & .17 & .09 & .04 & .01 & .01 & $3.2_{4}$ \\
\hline 636 & .59 & .18 & .11 & .04 & .08 & .12 & .06 & .03 & .01 & $1.1_{6}$ \\
\hline 637 & .25 & .21 & .15 & .09 & .24 & .06 & .04 & .01 & .01 & 1.69 \\
\hline 638 & .59 & .25 & .13 & .07 & .06 & .05 & .04 & .10 & .01 & $0.9_{5}$ \\
\hline 639 & .06 & .32 & .65 & .15 & .08 & .08 & .02 & .01 & .01 & $1.0_{0}$ \\
\hline
\end{tabular}

\section{Trace Element Standards}

The SRM's listed below were designed for trace chemical analysis, specifically for calibrating instruments and checking analytical techniques and procedures used to determine trace elements in various inorganic matrices. In addition many SRM's certified for chemical composition have one or more constituents certified or below the $100 \mu \mathrm{g} / \mathrm{g}$ level. Some SRM's in the following categories may be of use of in trace analytical work:

Steels (pages 18-30); High Purity Metals (page 41); Non ferrous alloys (pages 31-40); Environmental Standards (page 47); and Biological Standards (page 45). 


\begin{tabular}{|c|c|c|c|}
\hline SRM & Type-Matrix & Size & Unit of Issue \\
\hline 607 & Trace Elements in Feldspar............................. & & 5 gram. \\
\hline 608 & Trace Elements in Glass, Set & 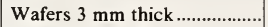 & Set: 2 each 614 and 616. \\
\hline 609 & 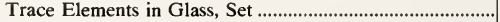 & Wafers $1 \mathrm{~mm}$ thick ................... & Set: 2 each 615 and 617. \\
\hline 610 & Trace Elements in Glass, 500 ppm & Wafers $3 \mathrm{~mm}$ thick ................ & 6 Wafers. \\
\hline 611 & 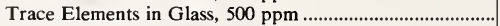 & Wafers $1 \mathrm{~mm}$ thick .................... & 6 Wafers. \\
\hline 612 & Trace Elements in Glass, 50 ppm & Wafers $3 \mathrm{~mm}$ thick .................... & 6 Wafers. \\
\hline 613 & Trace Elements in Glass, $50 \mathrm{ppm}$ & 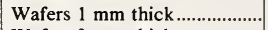 & 6 Wafers. \\
\hline 614 & Trace Elements in Glass, $1 \mathrm{ppm}$ & Wafers $3 \mathrm{~mm}$ thick................ & 6 Wafers. \\
\hline 615 & Trace Elements in Glass, $1 \mathrm{ppm}$ & 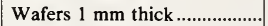 & 6 Wafers. \\
\hline 616 & Trace Elements in Glass, 0.02 ppm & 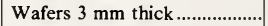 & 6 Wafers. \\
\hline 617 & Trace Elements in Glass, $0.02 \mathrm{ppm}$ & 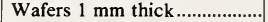 & 6 Wafers. \\
\hline 618 & Trace Elements in Glass, Set & 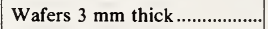 & Set: 1 each $610,612,614$ and 616 . \\
\hline 619 & Trace Elements in Glass, Set & Wafers $1 \mathrm{~mm}$ thick .................... & Set: 1 each $611,613,615$ and 617 . \\
\hline
\end{tabular}

NOTE: Glass-Nominal Composition: $72 \% \mathrm{SiO}_{2}, 12 \% \mathrm{CaO}, 14 \% \mathrm{Na}_{2} \mathrm{O}$, and $2 \% \mathrm{Al}_{2} \mathrm{O}_{3}$.

Trace Element Standards (Nominal Concentrations)

\begin{tabular}{|c|c|c|c|c|c|}
\hline Element & 607 (ppm) & $\begin{array}{c}610-611 \\
(\mathrm{ppm})\end{array}$ & $\begin{array}{c}612-613 \\
(\mathrm{ppm})\end{array}$ & $\begin{array}{c}614-615 \\
(\mathrm{ppm})\end{array}$ & $\begin{array}{c}616-617 \\
(\mathrm{ppm})\end{array}$ \\
\hline Antimony ................................ & & & & $(1.06)$ & $(0.078)$ \\
\hline Barium & ........................... & ........................... & $(41)$ & ......................... & .......................... \\
\hline Boron & .............................. & $(351)$ & (32) & $(1.30)$ & $(0.20)$ \\
\hline 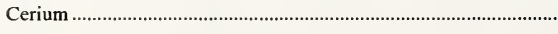 & & ....................... & (39) & & \\
\hline Cobalt ...................... & (........................... & $(390)$ & $(35.5)$ & 0.73 & \\
\hline Copper & ........................... & $(444)$ & $(37.7)$ & 1.37 & $(0.80)$ \\
\hline Dysprosium & (........................... & .......................... & $(35)$ & ........................... & (.......................... \\
\hline Erbium & & & (39) & (....................... & (....................... \\
\hline Europium......... & & & (36) & $(0.99)$ & 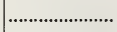 \\
\hline Gadolinium ................................ & (....................... & $\cdots \cdots \cdot$ & (39) & & (........................ \\
\hline Gallium & 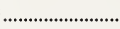 & ........................... & & (1.3) & $(0.23)$ \\
\hline Gold & (............................ & $(25)$ & (5) & $(0.5)$ & $(0.18)$ \\
\hline Iron & .................... & 458 & 51 & 13.5 & (11) \\
\hline Lanthanum & 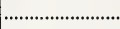 & & (36) & $(0.83)$ & $(0.034)$ \\
\hline Lead & 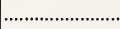 & 426 & 38.57 & 2.32 & 1.85 \\
\hline Manganese & .............................. & 485 & $(39.6)$ & & ........................... \\
\hline Neodymium.. & & & $(36)$ & & . \\
\hline 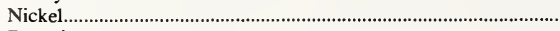 & 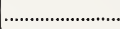 & 458.7 & 38.8 & $(0.95)$ & 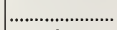 \\
\hline Potassium & ............................ & (461) & (64) & 30 & 29 \\
\hline Rubidium & 523.90 & 425.7 & 31.4 & 0.855 & 0.100 \\
\hline Samarium.. & & & (39) & & \\
\hline Scandium & ....................... & ................... & ..................... & $(0.59)$ & $(0.026)$ \\
\hline Silver & ........................... & $(254)$ & 22.0 & 0.42 & ................... \\
\hline Strontium & 65.485 & 515.5 & 78.4 & 45.8 & 41.72 \\
\hline , & $\ldots \ldots \ldots \ldots$ & $(61.8)$ & $(15.7)$ & $(0.269)$ & $(0.0082)$ \\
\hline Thorium & 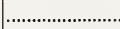 & 457.2 & 37.79 & 0.748 & 0.0252 \\
\hline Titanium & 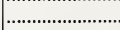 & $(437)$ & $(50.1)$ & (3.1) & $(2.5)$ \\
\hline Uranium & 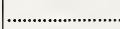 & 461.5 & 37.38 & 0.823 & 0.0721 \\
\hline Ytterbium & ............................ & ........................... & (42) & ….......... & ........................... \\
\hline Zinc & ............................... & $(433)$ & & ............. & ............................ \\
\hline
\end{tabular}

In addition to the 36 elements listed above, the Glass SRM's contain the following 25 elements: $\mathrm{As}, \mathrm{Be}, \mathrm{Bi}, \mathrm{Cs}, \mathrm{Cl}, \mathrm{F}, \mathrm{Ge}, \mathrm{Hf}, \mathrm{Hg}$, $\mathrm{Li}, \mathrm{Lu}, \mathrm{Mg}, \mathrm{Nb}, \mathrm{P}, \mathrm{Pr}, \mathrm{Se}, \mathrm{S}, \mathrm{Te}, \mathrm{Tb}, \mathrm{Tm}, \mathrm{Sn}, \mathrm{W}, \mathrm{V}, \mathrm{Y}$, and Zr. 


\section{Nuclear Materials}

\section{Special Nuclear Materials}

These SRM's are available to DOE contractors, NRC or State Licensees, and foreign governments that have entered an Agreement for Cooperation with the U.S. Government concerning the Civil Uses of Atomic Energy. The purchase request for these SRM's must be made on special forms obtainable from the Office of Standard Reference Materials, Room B311, Chemistry Building, National Bureau of Standards, Washington, D.C. 20234.

Plutonium Assay Standards

\begin{tabular}{|c|c|c|c|c|}
\hline SRM & Type & Certified for & Wt/unit (grams) & Purity (percent) \\
\hline 944 & Plutonium sulfate tetrahydrate & Plutonium Content......... & 0.5 & $* 47.50$ \\
\hline 945 & 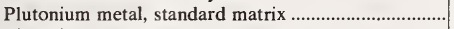 & 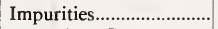 & 5 & $(99.9)$ \\
\hline $949 \mathrm{e}$ & 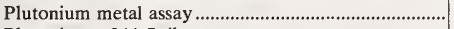 & Plutonium Content........ & $\dagger 0.5$ & 99.996 \\
\hline 996 & 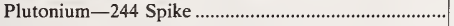 & IN PREP ......................... & & \\
\hline
\end{tabular}

*Stoichiometric †Nominal weight (Values in parentheses are not certified, but are given for information only.)

Plutonium Isotopic Standards

\begin{tabular}{|c|c|c|c|c|c|c|c|}
\hline \multirow{2}{*}{ SRM } & \multirow{2}{*}{ Type } & \multirow{2}{*}{$\begin{array}{l}\text { Wt/units } \\
\text { (grams) }\end{array}$} & \multicolumn{5}{|c|}{ Atom Percent } \\
\hline & & & ${ }^{238} \mathrm{Pu}$ & ${ }^{239} \mathrm{Pu}$ & ${ }^{240} \mathrm{Pu}$ & ${ }^{241} \mathrm{Pu}$ & ${ }^{242} \mathrm{Pu}$ \\
\hline 946 & Plutonium Sulfate Tetrohydrate................. & 0.25 & 0.247 & 83.128 & 12.069 & 3.991 & 0.565 \\
\hline 947 & Plutonium Sulfate Tetrohydrate................... & .25 & .296 & 75.696 & 18.288 & 4.540 & 1.180 \\
\hline 948 & Plutonium Sulfate Tetrohydrate................ & .25 & .011 & 91.574 & 7.914 & 0.468 & 0.0330 \\
\hline
\end{tabular}

Uranium Assay Standards

\begin{tabular}{|c|c|c|c|c|}
\hline SRM & Type & Certified for & $\begin{array}{c}\text { Wt/ } \\
\text { unit } \\
\text { (grams) }\end{array}$ & Purity (percent) \\
\hline $950 \mathrm{~b}$ & 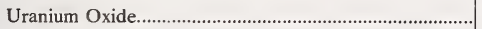 & Uranium Oxide.... & 25 & $99.968\left(\mathrm{U}_{3} \mathrm{O}_{8}\right)$ \\
\hline 960 & Uranium Metal & Uranium .................. & 26 & $99.975(\mathrm{U})$ \\
\hline 993 & 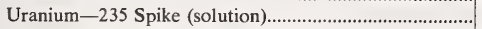 & Uranium ................... & 15 & $99.8195(\mathrm{U}-235)$ \\
\hline 995 & 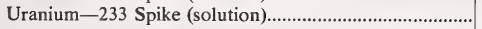 & Uranium ................. & 10 & $99.9245(\mathrm{U}-233)$ \\
\hline
\end{tabular}

Uranium Isotopic Standards

\begin{tabular}{|c|c|c|c|c|c|c|}
\hline \multirow{2}{*}{ SRM } & \multirow{2}{*}{ Uranium Oxide $\left(\mathrm{U}_{3} \mathrm{O}_{8}\right)$} & \multirow{2}{*}{$\begin{array}{c}\mathrm{Wt} \\
\text { (grams) }\end{array}$} & \multicolumn{4}{|c|}{ Atom Percent } \\
\hline & & & ${ }^{234} \mathrm{U}$ & ${ }^{235} \mathrm{U}$ & ${ }^{236} \mathrm{U}$ & ${ }^{238} \mathrm{U}$ \\
\hline $\mathrm{U}-0002$ & Depleted & 1.0 & 0.00016 & 0.01755 & $<0.00001$ & 99.9823 \\
\hline $\mathrm{U}-005$ & Depleted & 1.0 & .00218 & .4895 & .0046 & 99.504 \\
\hline U-010 & 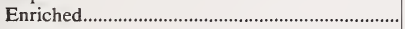 & 1.0 & .00541 & 1.0037 & .00681 & 98.984 \\
\hline $\mathrm{U}-015$ & Enriched & 1.0 & .00850 & 1.5323 & .0164 & 98.443 \\
\hline U-020 & 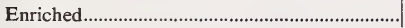 & 1.0 & .0125 & 2.038 & .0165 & 97.933 \\
\hline
\end{tabular}


Uranium Isotopic Standards-Continued

\begin{tabular}{|c|c|c|c|c|c|c|}
\hline \multirow{2}{*}{ SRM } & \multirow{2}{*}{ Uranium Oxide $\left(\mathrm{U}_{3} \mathrm{O}_{8}\right)$} & \multirow{2}{*}{$\underset{\text { (grams) }}{\mathrm{Wt}}$} & \multicolumn{4}{|c|}{ Atom Percent } \\
\hline & & & ${ }^{234} \mathrm{U}$ & ${ }^{235} \mathrm{U}$ & ${ }^{236} \mathrm{U}$ & ${ }^{238} \mathrm{U}$ \\
\hline U-030 & Enriched & 1.0 & .0190 & 3.046 & .0204 & 96.915 \\
\hline $\mathrm{U}-050$ & Enriched & 1.0 & .0279 & 5.010 & .0480 & 94.915 \\
\hline $\mathrm{U}-100$ & 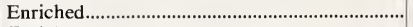 & 1.0 & .0676 & 10.190 & .0379 & 89.704 \\
\hline $\mathrm{U}-150$ & 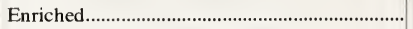 & 1.0 & .0993 & 15.307 & .0660 & 84.528 \\
\hline $\mathrm{U}-200$ & 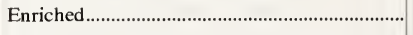 & 1.0 & .1246 & 20.013 & .2116 & 79.651 \\
\hline $\mathrm{U}-350$ & Enriched & 1.0 & .2498 & 35.190 & .1673 & 64.393 \\
\hline $\mathrm{U}-500$ & Enriched & 1.0 & .5181 & 49.696 & .0755 & 49.711 \\
\hline $\mathrm{U}-750$ & 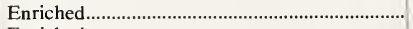 & 1.0 & .5923 & 75.357 & .2499 & 23.801 \\
\hline $\mathrm{U}-800$ & 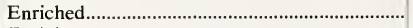 & 1.0 & .6563 & 80.279 & .2445 & 18.820 \\
\hline U-850 & 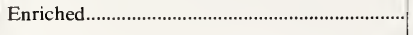 & 1.0 & .6437 & 85.137 & .3704 & 13.848 \\
\hline U-900 & 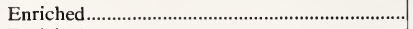 & 1.0 & .7777 & 90.196 & .3327 & 8.693 \\
\hline U-930 & 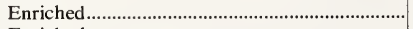 & 1.0 & 1.0812 & 93.336 & .2027 & 5.380 \\
\hline U-970 & Enriched & 1.0 & 1.6653 & 97.663 & .1491 & 0.5229 \\
\hline
\end{tabular}

\section{Neutron Density Standard}

This SRM is provided as a reference source of a cobalt-in-aluminum alloy to serve as a neutron density monitor wire SRM. Accurate determination of thermal neutron densities is essential in irradiation tests to obtain a basis for comparison of densities among reactors, in applying data in the design of reactors, in understanding the mechanisms of radiation damage, and for use in neutron activation analysis. The wire is $0.5 \mathrm{~mm}$ in diameter and 1 meter long.

\begin{tabular}{|c|c|c|}
\hline SRM & Type & $\begin{array}{l}\text { Cobalt Content } \\
\text { (Weight percent) }\end{array}$ \\
\hline 953 & Neutron density monitor wire (Co in A1) & 0.116 \\
\hline
\end{tabular}

\section{Fission Track Glass Standards}

These SRM's, at four uranium concentration levels, will aid fission track laboratories in interlaboratory comparisons of data and in monitoring neutron flux for irradiations. The fission track glass standards are certified for the neutron flux $\left(\mathrm{n} \cdot \mathrm{cm}^{-2} \cdot \mathrm{sec}^{-1}\right)$ that induced uranium fission in selected wafers. The materials were irradiated in the NBS 10 Megawatt Research Reactor, at two different neutron energies.

Each SRM unit contains four unirradiated glass wafers and two irradiated wafers.

\begin{tabular}{|c|c|c|c|}
\hline SRM & Total U concentration; ppm (by weight) & ${ }^{235} \mathrm{U}$ Atom Percent & Irradiation time (sec.) \\
\hline 961 & 461.5 & 0.2376 & $\begin{array}{lr}\text { RT-3 } & 8 \\
\text { RT-4 } & 12\end{array}$ \\
\hline $962 \mathrm{a}$ & 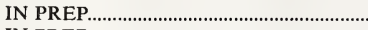 & 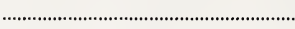 & (1) \\
\hline $963 a$ & 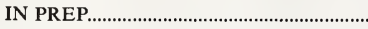 & 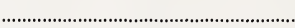 & \\
\hline 964 & 0.0721 & 0.616 & $\begin{array}{ll}\text { RT-3 } & 360 \\
\text { RT-4 } & 540\end{array}$ \\
\hline
\end{tabular}


The isotopic composition of these SRM's has been determined by mass spectrometry, by comparison with mixtures prepared from high-purity separated isotopes: They are useful for those looking for small variations in the isotopic composition of the elements, and for the evaluation of mass discrimination effects encountered in the operation of mass spectrometers.

\begin{tabular}{|c|c|c|c|}
\hline SRM & Isotopic Reference Standards & Element Certified & $\mathrm{Wt} /$ Unit (grams) \\
\hline 951 & 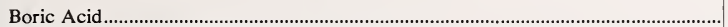 & Boron & 100 \\
\hline 952 & Boric Acid, 95\% Enriched ${ }^{10} \mathrm{~B}$ & Boron & 0.25 \\
\hline 975 & Sodium Chloride & Chlorine & .25 \\
\hline 976 & Copper Metal & Copper & .25 \\
\hline 977 & 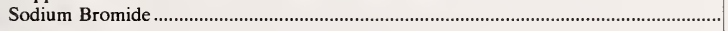 & Bromine & .25 \\
\hline 978 & Silver Nitrate ......... & Silver & .25 \\
\hline 979 & Chromium Nitrate.. & Chromium & .25 \\
\hline 980 & 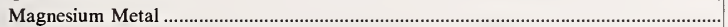 & Magenesium & .25 \\
\hline *981 & 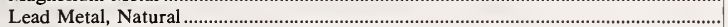 & Lead & 1.0 \\
\hline *982 & Lead Metal, Equal Atom $(206 / 208)$ & Lead & 1.0 \\
\hline *983 & Lead Metal, Radiogenic $(92 \%-206)$ & Lead & 1.0 \\
\hline 984 & 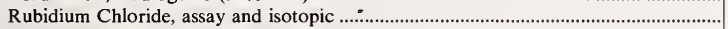 & Rubidium & 0.25 \\
\hline 987 & 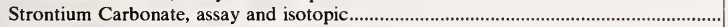 & Strontium & 1.0 \\
\hline 989 & Rhenium, assay and isotopic & Rhenium & pkg. (50) \\
\hline 990 & 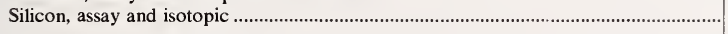 & Silicon & wafer, 3 \\
\hline & & & $\begin{array}{l}\mathrm{cm} \mathrm{D} \\
\times 0.2 \mathrm{~cm}\end{array}$ \\
\hline 991 & Lead-206 Spike, assay and isotopic & Lead & 15 \\
\hline **993 & 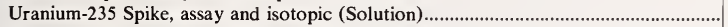 & Uranium & 15 \\
\hline 995 & Uranium-233 Spike, assay and isotopic (Solution) & Uranium & 10 \\
\hline
\end{tabular}

*Sold as a set only of three 981,982 , and 983 .

**Special Nuclear forms required.

\section{CERTIFIED PHYSICAL PROPERTIES STANDARDS}

\section{Ion Activity Standards}

These SRM's are intended for use in the preparation of solutions for the calibration of specification electrodes. This include the $\mathrm{pH}$ and $\mathrm{pD}$ measuring systems.

\section{pH Standards}

These SRM's are furnished as crystals for the preparation of solutions of known hydrogen ion concentration for calibrating and checking the performance of commercially available $\mathrm{pH}$ materials and instruments. They are furnished with certificates giving directions for preparation of the solutions and tables of $\mathrm{pH}$ values at various temperatures.

SRM's 186Ic and 186IIc, 191 and 192, and 922 and 923, are certified for use in admixture only. At an equimolar (0.025 molal) mixture of SRM's 186Ic and 186IIc, a pH(S) of 6.863 at $25^{\circ} \mathrm{C}$ is obtained. Directions also are furnished for the preparation of a physiological reference solution from 186Ic and 186IIc having a $\mathrm{pH}(\mathrm{S})$ of 7.415 at $25^{\circ} \mathrm{C}$. 


\begin{tabular}{|c|c|c|c|}
\hline SRM & Type & $\begin{array}{c}\mathrm{pH}(\mathrm{S})(\text { at } 25 \\
\left.{ }^{\circ} \mathrm{C}\right)\end{array}$ & $\begin{array}{l}\text { Wt/Uni } \\
\text { (grams) }\end{array}$ \\
\hline $185 \mathrm{e}$ & Postassium acid phthalate ...................... & 4.004 & 60 \\
\hline $186 \mathrm{Ic}$ & Postassium dihydrogen phosphate & 6.8631 & 30 \\
\hline 186IIc & Disodium hydrogen phosphate $\}$ & $7.415\}$ & 30 \\
\hline $187 \mathrm{~b}$ & Borax & 9.183 & 30 \\
\hline 188 & 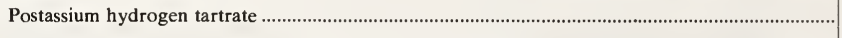 & 3.557 & 60 \\
\hline 189 & Postassium tetroxalate ........................................ & 1.679 & 65 \\
\hline 191 & Sodium bicarbonate & & 130 \\
\hline 192 & 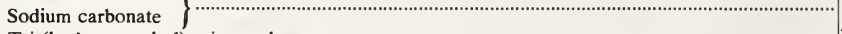 & 10.01 & $\left\{\begin{array}{l}30 \\
30\end{array}\right.$ \\
\hline 922 & Tris(hydroxymethyl)aminomethane & & 25 \\
\hline 923 & Tris(hydroxymethyl)aminomethane hydrochloride $\}$ & 7.699 & $\left\{\begin{array}{l}35 \\
35\end{array}\right.$ \\
\hline
\end{tabular}

\section{PD Standards}

These SRM's are furnished as crystals for preparation of solutions of known deuterium-ion concentration for the calibration and correction of $\mathrm{pH}$ indicating equipment to indicate pD data. SRM's 2186I and 2186II, and 2191 and 2192, are certified for use in admixtures only.

\begin{tabular}{|c|c|c|c|}
\hline SRM & Type & $\begin{array}{l}\mathrm{pD}(\mathrm{S}) \\
\text { Values }\end{array}$ & $\begin{array}{l}\text { Wt/Unit } \\
\text { (grams) }\end{array}$ \\
\hline $2186 \mathrm{I}$ & Potassium dihydrogen phosphate & \multirow{4}{*}{$\begin{array}{r}7.43 \\
10.74\end{array}$} & \multirow{4}{*}{$\begin{array}{l}\left\{\begin{array}{l}30 \\
30\end{array}\right. \\
\left\{\begin{array}{l}30 \\
30\end{array}\right.\end{array}$} \\
\hline $2186 \mathrm{II}$ & Disodium hydrogen phosphate & & \\
\hline $\begin{array}{l}2191 \\
2192\end{array}$ & Sodium bicarbonate & & \\
\hline 2192 & Sodium carbonate $\} \cdots$ & & \\
\hline
\end{tabular}

\section{Ion-Selective Electrodes}

These SRM's are certified for the calibration of ion-selective electrodes and have conventional ionic activities based on the Stokes-Robinson hydration theory for ionic strengths greater than 0.1 mole per liter.

\begin{tabular}{|c|c|c|c|}
\hline SRM & Type & Certified Property & $\begin{array}{l}\text { Wt/Unit } \\
\text { (grams) }\end{array}$ \\
\hline 2201 & 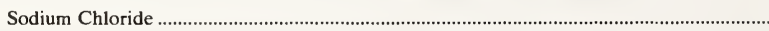 & $\mathrm{pNa}, \mathrm{pCl}$ & 125 \\
\hline 2202 & Potassium Chloride & $\mathrm{pK}, \mathrm{pCl}$ & 160 \\
\hline 2203 & 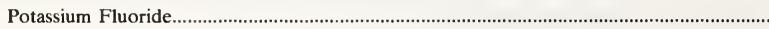 & $\mathrm{pF}$ & 125 \\
\hline
\end{tabular}

\section{Mechanical and Metrology Standards}

\section{Scanning Electron Microscope Standards}

These SRM's are for use in calibrating the magnification scale and for evaluating the resolution and performance of an SEM. SRM 484b has spacings of 1, 2, 3, 5 and $50 \mathrm{~mm}$ and can be used to calibrate the magnification scale of an SEM from 1000 to $20,000 \mathrm{X}$ to an accuracy of 5 percent or better. This standard is a metallographic cross-section of alternate layers of electro deposited gold and nickel, encapsulated in copper-filled epoxy, and mounted within a section of stainless steel tubing. If the surface of this SRM were etched by de sputtering, it would be useful for calibrating optical microscopes. SRM 469 is a small bead (about $5 \mathrm{~mm}$ in diameter) of an aluminum-tungsten alloy that has a fine dendritic surface suitable for both high and low resolution testing. This structure has high secondary electron emission, high contrast, and spacings varying from about 20 to $200 \mathrm{~nm}$. This bead is non-magnetic, vacuum clean, has no adverse reaction in the electron beam, needs no surface preparation or coating, and can be used repeatedly. Extracted replicas of the dendritic structure have been used to test transmission electron microscopes (TEM). 


\begin{tabular}{|c|c|c|c|}
\hline SRM & Type & Spacings & Size \\
\hline $\begin{array}{l}469 \\
484 \mathrm{~b}\end{array}$ & $\begin{array}{l}\text { SEM Resolution Test Specimen } \\
\text { SEM Magnification Standard }\end{array}$ & $\begin{array}{l}0.02 \text { to } 0.2 \mathrm{~mm} \\
1,2,3,5 \text { and } 50 \mathrm{~mm}\end{array}$ & $\begin{array}{l}5-\mathrm{mm} \text { bead } \\
6.5 \times 11 \mathrm{~mm} \text { tube }\end{array}$ \\
\hline
\end{tabular}

\section{Optical Microscope Linewidth-Measurement Standard}

This SRM is for use in calibrating optical microscopes used to measure the widths of opaque lines and clear spaces on integrated-circuit photomasks. It can also be used to calibrate line spacings and line-tospace ratios. The accuracy of a measured linewidth or line spacing on SRM 474 is $\pm 0.05 \mathrm{~mm}$ or better. Recommended procedures are provided with each SRM for the adjustment and calibration of measurement systems using transmitted illumination, including filar, image shearing, and video micrometer. SRM 474 is an anti-reflective chromium photoplate on a borosilicate glass substrate. This SRM is not recommended for use with partially transmitting materials or in reflected light with opaque materials and it should not be used in a scanning electron microscope.

\begin{tabular}{c|c|c|c}
\hline \multicolumn{1}{c|}{ SRM } & \multicolumn{1}{|c|}{ Type } & Spacings & \multicolumn{1}{c}{ Size } \\
\hline 474 & Linewidth Measurement Standard.......................................... 0.5 to $10 \mathrm{~mm}$ & $6.4 \times 6.4 \times 0.2 \mathrm{~cm}$. \\
\hline
\end{tabular}

\section{Coating Thickness Standards}

These SRM's have a specimen size of $3 \times 3 \mathrm{~cm}$ and are for calibrating coating thickness gages of the magnetic type for the measurement of thickness of nonmagnetic coatings on steel, nickel on steel, or nickel on nonmagnetic substrates. The steel substrates have the magnetic properties of AISI 1010 steel and the nickel coatings have the magnetic properties of an annealed, Watts nickel electrodeposit free of cobalt and iron.

The magnetic type thickness gages are often used to measure the thickness of paint and other organic coatings on steel, as well as zinc (galvanized) and other nonmagnetic metallic coatings. The number of different thicknesses required for these calibrations depends on the type of gage and the coating thicknesses to be measured.

The magnetic type thickness gages can be used to estimate magnetic properties of austenitic stainless steel weld metal. Because the magnetic properties of the weld metal are closely related to the ferrite content of the weld, these instruments are used to estimate the ferrite content. For these measurements, the coating thickness SRM 1370a is used to calibrate the instrument. The ferrite contents having magnetic properties similar to those of the various coating thickness SRM's have been established by other laboratories.

SRM's with gold and tin coatings on various substrates have a specimen size of $15 \mathrm{X} 15 \mathrm{~mm}$ and are for calibrating coating thickness gages of the beta-backscatter type and for calibrating $\mathrm{x}$-ray fluorescence methods for the measurement of the weight per unit area of gold or tin coatings.

The gold coating standards were measured by beta-ray backscatter and $\mathrm{x}$-ray fluorescence techniques relative to NBS gold coating materials for which the average weights per unit area were determined by weight and area measurements. They are suitable for the direct calibration of equipment used to measure weight per unit area of gold coating of equivalent purity. From the density and weight per unit area, the instruments can be calibrated in terms of the thickness of the standard.

For the tin coating standards, x-ray fluorescence techniques were used to measure the thickness of the tin coating relative to NBS tin coating material for which the average weights per unit area were determined by weight and area measurements. 
Instrumental methods of measuring coating thickness are set forth in the following ASTM Methods of Test:

B244 Measuring Thickness of Anodic Coatings on Aluminum with Eddy-Current Instruments. B487 Measurement of Metal and Oxide Coating Thicknesses by Microscopical Examination of a Cross Section.

B499 Measurement of Coating Thicknesses by the Magnetic Method: Nonmagnetic Coatings on Magnetic Basis Metals.

B504 Measuring the Thicknesses of Metallic Coatings by the Coulometric Method.

B530 Measurement of Coating Thicknesses by the Magnetic Method: Electrodeposited Nickel Coatings on Magnetic and Nonmagnetic Substrates.

B567 Measurement of Coating Thickness by the Beta Backscatter Method.

B568 Measurement of Coating Thickness by X-Ray Spectrometry.

E376 Recommended Practice for Measuring Coatings on Thickness by Magnetic-Field or EddyCurrent (electro-magnetic) Test Methods.

D1186 Measurement of Dry Film Thickness of Nonmagnetic Organic Coatings Applied on a Magnetic Base.

D1400 Measurement of Dry Film Thickness of Nonmetallic Coatings of Paint, Varnish, Lacquer, and Related Products Applied on a Nonmagnetic Metal Base.

G12 Nondestructive Measurement of Film Thickness of Pipeline Coatings on Steel.

Nonmagnetic Coating on Magnetic Substrate (Copper and Chromium on Steel).

\begin{tabular}{|c|c|c|c|}
\hline \multirow{2}{*}{ SRM } & \multirow{2}{*}{ Unit Size } & \multicolumn{2}{|c|}{ Nominal Coating Thickness- } \\
\hline & & micrometer & milliinch (mil) \\
\hline $1301 \mathrm{a}$ & Set of 4 & 2.5 & 0.10 \\
\hline $1302 \mathrm{a}$ & Set of 4 & 6 & .24 \\
\hline $1303 \mathrm{a}$ & Set of 4 & 12 & .5 \\
\hline $1304 \mathrm{a}$ & Set of 4 & 20 & .8 \\
\hline $1305 \mathrm{a}$ & Set of 4 & 25 & 1.0 \\
\hline $1306 \mathrm{a}$ & Set of 4 & 40 & 1.6 \\
\hline $1307 \mathrm{a}$ & Set of 4 & 50 & 2.0 \\
\hline $1308 \mathrm{a}$ & Set of 4 & 65 & 2.6 \\
\hline $1310 \mathrm{a}$ & Set of 4 & 80 & 3.1 \\
\hline $1311 \mathrm{a}$ & Set of 4 & 140 & 5.5 \\
\hline $1312 \mathrm{a}$ & Set of 4 & 200 & 7.9 \\
\hline $1313 \mathrm{a}$ & Set of 4 & 250 & 9.8 \\
\hline $1314 \mathrm{a}$ & Set of 4 & 400 & 16 \\
\hline \multirow[t]{2}{*}{$1351 \mathrm{a}$} & Set of $4: 2$ each & 50 & 2.0 \\
\hline & & 40 & 5.5 \\
\hline \multirow[t]{4}{*}{$1361 \mathrm{a}$} & Set of 4 & 6 & 0.24 \\
\hline & & 12 & .5 \\
\hline & & 25 & 1.0 \\
\hline & & 50 & 2.0 \\
\hline \multirow[t]{4}{*}{$1362 \mathrm{a}$} & Set of 4 & 40 & 1.6 \\
\hline & & 80 & 3.1 \\
\hline & & 140 & 5.5 \\
\hline & & 200 & 7.9 \\
\hline \multirow[t]{4}{*}{$1363 \mathrm{a}$} & Set of 4 & 250 & 9.8 \\
\hline & & 400 & 16 \\
\hline & & 500 & 20 \\
\hline & & 650 & 26 \\
\hline
\end{tabular}




\begin{tabular}{|c|c|c|c|}
\hline \multirow{2}{*}{ SRM } & \multirow{2}{*}{ Unit Size } & \multicolumn{2}{|c|}{ Nominal Coating Thickness- } \\
\hline & & micrometer & milliinch (mil) \\
\hline $1364 \mathrm{a}$ & Set of 4 & $\begin{array}{r}820 \\
1000 \\
1500 \\
2000\end{array}$ & $\begin{array}{l}32 \\
39 \\
59 \\
79\end{array}$ \\
\hline $1370 \mathrm{a}$ & Set of 8 & $\begin{array}{r}200 \\
250 \\
400 \\
500 \\
650 \\
820 \\
1000 \\
1500\end{array}$ & $\begin{array}{c}7.9 \\
9.8 \\
16 \\
20 \\
26 \\
32 \\
39 \\
59\end{array}$ \\
\hline
\end{tabular}

Magnetic Coating on Magnetic Substrate (Nickel on Steel)

\begin{tabular}{|c|c|c|c|}
\hline \multirow{2}{*}{ SRM } & \multirow{2}{*}{ Unit Size } & \multicolumn{2}{|c|}{ Nominal Coating Thickness } \\
\hline & & micrometer & milliinch (mil) \\
\hline 1352a & \multirow{3}{*}{$\begin{array}{l}\text { Set of } 4: 2 \text { each } \\
\text { Set of } 4: 2 \text { each } \\
\text { Set of } 4: 1 \text { each }\end{array}$} & 9 & 0.35 \\
\hline $1353 \mathrm{a}$ & & 25 & 1.0 \\
\hline \multirow{4}{*}{$1365 \mathrm{a}$} & & 60 & 2.4 \\
\hline & & 9 & .4 \\
\hline & & 15 & .6 \\
\hline & Set of 4.1 ech & 20 & .8 \\
\hline \multirow{3}{*}{ 1366a } & 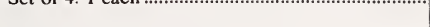 & 35 & 1.4 \\
\hline & & 40 & 1.6 \\
\hline & & 50 & 2.0 \\
\hline
\end{tabular}

Magnetic Coating on Non-Magnetic Substrate (Nickel and Chromium on Brass)

\begin{tabular}{|c|c|c|c|}
\hline \multirow{2}{*}{ SRM } & \multirow{2}{*}{ Unit Size } & \multicolumn{2}{|c|}{ Nominal Coating Thickness } \\
\hline & & micrometer & milliinch (mil) \\
\hline $1367 \mathrm{a} \ldots \ldots \ldots \ldots \ldots \ldots \ldots \ldots$ & Set of 4: 1 each & $\begin{array}{r}3 \\
9 \\
16 \\
25\end{array}$ & $\begin{array}{c}0.12 \\
.35 \\
.6 \\
1.0\end{array}$ \\
\hline
\end{tabular}

Gold Coating on Glass Sealing Alloy-ASTM Designation F15; Fe-53, Ni-29, and Co-17

\begin{tabular}{|c|c|c|c|c|}
\hline \multirow{2}{*}{ SRM } & \multirow{2}{*}{ Unit Size } & \multirow{2}{*}{$\begin{array}{c}\text { Nominal } \\
\text { Coating } \\
\text { Weight } \\
\left(\mathrm{mg} / \mathrm{cm}^{2}\right)\end{array}$} & \multicolumn{2}{|c|}{$\begin{array}{l}\text { Nominal Coating } \\
\text { Thickness }\end{array}$} \\
\hline & & & micrometer & microinch \\
\hline $1398 \mathrm{a}$ & Set of $4: 1$ each & $\begin{array}{r}1.5 \\
3.0 \\
6.0 \\
14.0\end{array}$ & $\begin{array}{l}0.8 \\
1.5 \\
3 \\
7\end{array}$ & $\begin{array}{r}30 \\
60 \\
120 \\
280\end{array}$ \\
\hline
\end{tabular}


Gold Coating on Nickel

\begin{tabular}{|c|c|c|c|c|}
\hline \multirow[t]{2}{*}{ SRM } & \multirow{2}{*}{ Unit Size } & \multirow{2}{*}{$\begin{array}{c}\text { Nominal } \\
\text { Coating } \\
\text { Weight } \\
\left(\mathrm{mg} / \mathrm{cm}^{2}\right)\end{array}$} & \multicolumn{2}{|c|}{$\begin{array}{l}\text { Nominal Coating } \\
\text { Thickness }\end{array}$} \\
\hline & & & micrometer & microinch \\
\hline $1384 a$ & Set of $4: 2$ each & $\begin{array}{l}1.5 \\
3.0\end{array}$ & $\begin{array}{l}0.8 \\
1.5\end{array}$ & $\begin{array}{l}30 \\
60\end{array}$ \\
\hline $1399 \mathrm{a}$ & 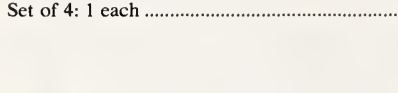 & $\begin{array}{r}1.5 \\
3.0 \\
6.0 \\
17.0\end{array}$ & $\begin{array}{l}0.8 \\
1.5 \\
3 \\
9\end{array}$ & $\begin{array}{r}30 \\
60 \\
120 \\
350\end{array}$ \\
\hline
\end{tabular}

Gold Coating on Copper-Clad, Glass-Epoxy Laminate

\begin{tabular}{|c|c|c|c|c|}
\hline \multirow{2}{*}{ SRM } & \multirow{2}{*}{ Unit Size } & \multirow{2}{*}{$\begin{array}{c}\text { Nominal } \\
\text { Coating } \\
\text { Weight } \\
\left(\mathrm{mg} / \mathrm{cm}^{2}\right)\end{array}$} & \multicolumn{2}{|c|}{$\begin{array}{l}\text { Nominal Coating } \\
\text { Thickness }\end{array}$} \\
\hline & & & micrometer & microinch \\
\hline $2308 \mathrm{a}$ & Set of $4: 1$ each & $\begin{array}{r}1.5 \\
3.0 \\
6.0 \\
14.0\end{array}$ & $\begin{array}{l}0.8 \\
1.5 \\
3 \\
7\end{array}$ & $\begin{array}{r}30 \\
60 \\
120 \\
280\end{array}$ \\
\hline
\end{tabular}

\section{Gold Coating on Copper}

\begin{tabular}{|c|c|c|c|c|}
\hline \multirow{2}{*}{ SRM } & \multirow{2}{*}{ Unit Size } & \multirow{2}{*}{$\begin{array}{c}\text { Nominal } \\
\text { Coating } \\
\text { Weight } \\
\left(\mathrm{mg} / \mathrm{cm}^{2}\right)\end{array}$} & \multicolumn{2}{|c|}{$\begin{array}{c}\text { Nominal Coating } \\
\text { Thickness }\end{array}$} \\
\hline & & & micrometer & microinch \\
\hline $2318 a$ & Set of $4: 1$ each & $\begin{array}{r}1.5 \\
3.0 \\
6.0 \\
14.0\end{array}$ & $\begin{array}{l}0.8 \\
1.5 \\
3 \\
7\end{array}$ & $\begin{array}{r}30 \\
60 \\
120 \\
280\end{array}$ \\
\hline
\end{tabular}

Tin Coating on Steel

\begin{tabular}{|c|c|c|c|c|}
\hline \multirow[t]{2}{*}{ SRM } & \multirow{2}{*}{ Unit Size } & \multirow{2}{*}{$\begin{array}{c}\text { Nominal } \\
\text { Coating } \\
\text { Weight } \\
\left(\mathrm{mg} / \mathrm{cm}^{2}\right)\end{array}$} & \multicolumn{2}{|c|}{$\begin{array}{c}\text { Nominal Coating } \\
\text { Thickness }\end{array}$} \\
\hline & & & micrometer & microinch \\
\hline $2338 \mathrm{a}$ & Set of $4: 2$ each & $\begin{array}{r}2.0 \\
12.0\end{array}$ & $\begin{array}{r}2.8 \\
16.5\end{array}$ & $\begin{array}{l}110 \\
650\end{array}$ \\
\hline $2339 \mathrm{a}$ & Set of $4: 1$ each & $\begin{array}{r}1.1 \\
3.0 \\
5.0 \\
14.0\end{array}$ & $\begin{array}{l}1.5 \\
4.1 \\
7 \\
19\end{array}$ & $\begin{array}{r}60 \\
160 \\
280 \\
750\end{array}$ \\
\hline
\end{tabular}


Glass Standards

\begin{tabular}{|c|c|c|}
\hline SRM & Type & Unit of Issue \\
\hline 622 & Soda-lime-silica & $2.2 \mathrm{~kg}$. \\
\hline 623 & Borosilicate & $2.2 \mathrm{~kg}$. \\
\hline 624 & Lead-silica, for de resistivity & $200 \mathrm{~g}$. \\
\hline 708 & 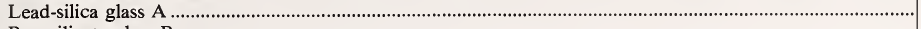 & $625 \mathrm{~g}$. \\
\hline & Borosilicate glass B & $275 \mathrm{~g}$. \\
\hline 709 & Extra dense lead & $500 \mathrm{~g}$. \\
\hline 710 & 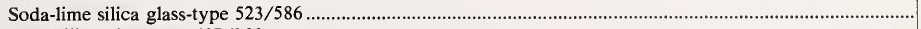 & $2 \mathrm{lb}$. \\
\hline 711 & Lead-silica glass-type $617 / 366$ & $3 \mathrm{lb}$. \\
\hline 712 & Mixed alkalai lead silicate glass, $1 / 4$ in patties ( 6 pcs.) & $0.5 \mathrm{lb}$. \\
\hline 713 & 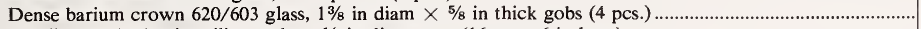 & $.5 \mathrm{lb}$ \\
\hline 714 & Alkaline earth alumina silicate glass, $1 / 4$ in diam cane $(16 \mathrm{pcs}-6$ in long) & $.5 \mathrm{lb}$. \\
\hline 715 & Alkalai-free aluminosilicate glass, $1 / 4$ in diam cane $(13$ pcs -6 in long) & $200 \mathrm{~g}$ \\
\hline 716 & Neutral (borosilicate) glass, $1 / 2$ in diam cane $(6 \mathrm{pcs}-6$ in long) & $250 \mathrm{~g}$. \\
\hline 717 & 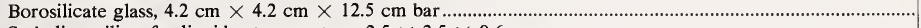 & $500 \mathrm{~g}$. \\
\hline 723 & 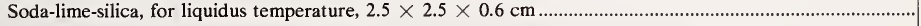 & $60 \mathrm{~g}$. \\
\hline
\end{tabular}

\section{Chemical Resistance (Durability) of Glass}

These SRM's are certified for use in checking test methods and for calibrating equipment used to determine the resistance of glass containers to chemical attack. The values given in the table represent the volume of fiftieth-normal sulfuric acid used to titrate to the methyl-red end point the alkaline extract from a crushed sample of glass after exposure to high-purity water at $121{ }^{\circ} \mathrm{C}$.

\begin{tabular}{|c|c|c|}
\hline SRM & Type & $\begin{array}{l}\mathrm{mL} \text { of } \mathrm{N} / \\
50 \mathrm{H}_{2} \mathrm{SO}_{4}\end{array}$ \\
\hline $622 \ldots \ldots \ldots$ & Soda-lime-silica & 7.67 \\
\hline $623 \ldots \ldots \ldots$ & Borosilicate & 0.34 \\
\hline
\end{tabular}

\section{Electrical Volume Resistivity}

This SRM is certified for use in checking test methods and for calibrating equipment used to determine the dc volume resistivity of glass in accordance with ASTM C 657-72.

\begin{tabular}{|c|c|c|c|}
\hline SRM & Type & $\log _{10}$ Electrical Volume Resistivity & $\begin{array}{l}\text { Tempera- } \\
\text { ture, }{ }^{\circ} \mathrm{C}\end{array}$ \\
\hline 624 & 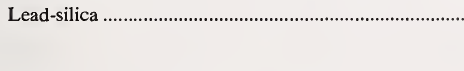 & $\begin{array}{r}11.07 \Omega \cdot \mathrm{cm} \\
9.88 \Omega \\
8.88 \Omega \\
\Omega \cdot \mathrm{cm}\end{array}$ & $\begin{array}{l}250 \\
300 \\
350\end{array}$ \\
\hline
\end{tabular}

\section{Glass Viscosity Standards}

SRM's 710, 711, and 717 are furnished as rectangular-shaped bars, and are certified for viscosity between values of $10^{2}$ and $10^{12}$ poises. They are furnished to check the performance of high-temperature viscosity equipment (rotating cylinders) and low-temperature viscosity equipment (fiber elongation, beambending, parallel-plates, etc.) 


\begin{tabular}{|c|c|c|c|c|c|c|c|c|c|c|c|}
\hline \multirow{2}{*}{ SRM } & \multicolumn{11}{|c|}{ Temperature $\left({ }^{\circ} \mathrm{C}\right)$ at Viscosity (poises) } \\
\hline & $10^{2}$ & $10^{3}$ & $10^{4}$ & $10^{5}$ & $10^{8}$ & $10^{7}$ & $10^{8}$ & $10^{\circ}$ & $10^{10}$ & $10^{11}$ & $10^{12}$ \\
\hline 710 & 1434.3 & 1181.7 & 1019.0 & 905.3 & 821.5 & 757.1 & 706.1 & 664.7 & 630.4 & 601.5 & 576.9 \\
\hline 711 & 1327.1 & 1072.8 & 909.0 & 794.7 & 710.4 & 645.6 & 594.3 & 552.7 & 518.2 & 489.2 & 464.5 \\
\hline 717 & 1545.1 & 1248.8 & 1059.4 & 927.9 & 831.2 & 757.1 & 698.6 & 651.1 & 611.9 & 579.0 & 550.9 \\
\hline
\end{tabular}

\section{Glass Viscosity Fixpoints}

\begin{tabular}{|c|c|c|c|c|}
\hline SRM & Type of Glass & $\begin{array}{l}\text { Softening } \\
\text { Point }{ }^{\circ} \mathrm{C}\end{array}$ & $\begin{array}{l}\text { Annealing } \\
\text { Point }{ }^{\circ} \mathrm{C}\end{array}$ & $\begin{array}{c}\text { Strain } \\
\text { Point }{ }^{\circ} \mathrm{C}\end{array}$ \\
\hline 709 & Extra Dense Lead ............................. & 384 & 328 & 311 \\
\hline 710 & 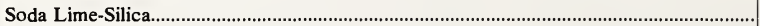 & 724 & 546 & 504 \\
\hline 711 & 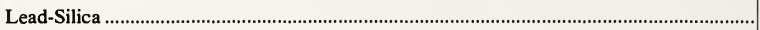 & 602 & 432 & 392 \\
\hline 712 & 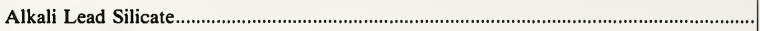 & 528 & 386 & 352 \\
\hline 713 & 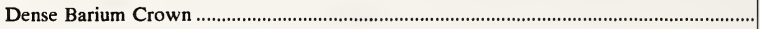 & 738 & 631 & 599 \\
\hline 714 & 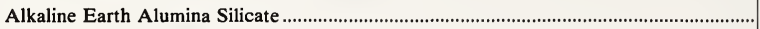 & 908 & 710 & 662 \\
\hline 715 & 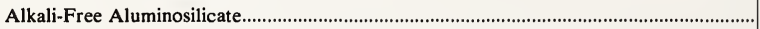 & 961 & 764 & 714 \\
\hline 716 & 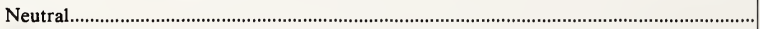 & 794 & 574 & 530 \\
\hline 717 & Borosilicate & 720 & 516 & 471 \\
\hline
\end{tabular}

\section{Relative Stress Optical Coefficient}

Three glasses have been certified for relative stress optical coefficient. These glasses will be used to check calibrations of instruments to measure this property, especially by the methods of test proposed by ASTM C770-73T. The glasses are in rectangular-shaped bars.

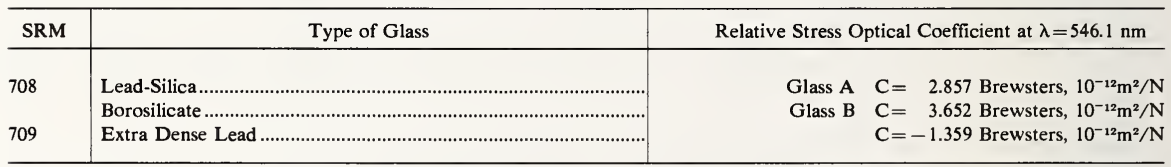

\section{Glass Liquidus Temperature}

This SRM is certified for use in checking test methods and for calibrating equipment used to determine the liquidus temperature of glass by the gradient furnace methods in accordance with ASTM C82976.

\begin{tabular}{|c|c|c|}
\hline SRM & Type & Temperature, ${ }^{\circ} \mathrm{C}$ \\
\hline 773 & Soda-lime-silica & 990 \\
\hline
\end{tabular}


This SRM is polycrystalline alumina prepared from a single block of material by isostatically cold pressing and then sintering alumina powder containing 0.1 percent magnesium oxide. It is intended for the calibration of apparatus used in the measurement of resonance frequencies from which elastic moduli are calculated. Each bar has been individually measured and calibrated, and all surfaces were machined flat and parallel.

\begin{tabular}{|c|c|c|}
\hline SRM & Type & Size \\
\hline 718 & Polycrystalline Alumina & $12.7 \times 1.27 \times 0.32 \mathrm{~cm}$ \\
\hline
\end{tabular}

\section{Density Standards}

SRM's $211 \mathrm{c}$ and $217 \mathrm{c}$ are certified for density (air saturated at $1 \mathrm{~atm}$ ) at 20,25, and $30^{\circ} \mathrm{C}$; and may be used to calibrate pycnometers and density balances. [See also, Refractive Index, page 77, and Combustion Calorimetric Standards, page 71.]

\begin{tabular}{|c|c|c|c|}
\hline SRM & Type & $\begin{array}{l}\text { Density } 20^{\circ} \mathrm{C} \\
(\mathrm{g} / \mathrm{mL})\end{array}$ & Amount, mL \\
\hline $\begin{array}{l}211 c \\
217 c-5\end{array}$ & Toluene Trimethlypentane & $\begin{array}{l}0.867 \\
0.692\end{array}$ & $\begin{array}{l}\text { IN PREP } \\
\text { IN PREP }\end{array}$ \\
\hline
\end{tabular}

\section{Polymer Standards}

These materials are certified for the properties indicated in the table, such as weight and number average molecular weight, molecular weight distribution, limiting viscosity numbers (intrinsic viscosities) in several solvents, density, and melt flow.

These SRM's have wide application not only in the calibration of instruments used in polymer characterization, such as light scattering photometers, osmometers, gel permeation chromatographs, but also wherever a well characterized polymer material is needed, as for example in studies of dilute solution behavior, rheology, and polymer crystal physics.

SRM 1475 is accompanied by a series of papers, reprinted from the Journal of Research of the National Bureau of Standards, which describe how the measurements were obtained.

\begin{tabular}{|c|c|c|}
\hline SRM & Type & $\begin{array}{l}\text { Wt/Unit } \\
\text { (grams) }\end{array}$ \\
\hline 705 & 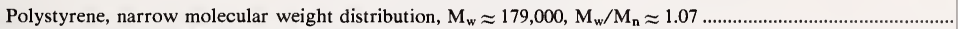 & 5 \\
\hline 706 & 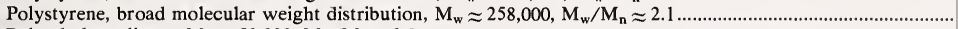 & 18 \\
\hline 1475 & Polyethylene, linear, $M_{w} \approx 52,000, M_{w} / M_{n} \approx 2.9$ & 50 \\
\hline 1476 & Polyethylene, branched & 50 \\
\hline 1478 & 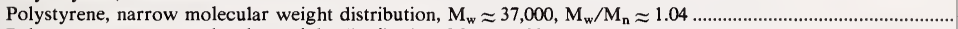 & 2 \\
\hline 1479 & 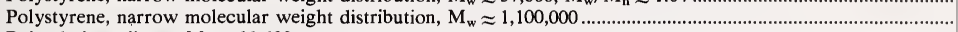 & 2 \\
\hline 1482 & Polyethylene, linear, $\mathbf{M}_{\mathrm{w}} \approx 13,600$ & 2 \\
\hline 1483 & Polyethylene, linear, $\mathbf{M}_{\mathrm{w}} \approx 32,100$ & 5 \\
\hline 1484 & Polyethylene, linear, $M_{w} \approx 119,600$ & 2 \\
\hline
\end{tabular}


The following table lists the properties (and method) certified for these SRM's.

\begin{tabular}{|c|c|c|c|c|c|c|c|c|c|c|}
\hline Property & Method & 705 & 706 & 1475 & 1476 & 1478 & 1479 & 1482 & 1483 & 1484 \\
\hline \multirow[t]{4}{*}{ 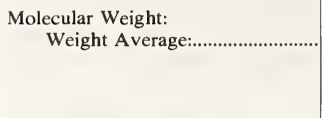 } & (Light Scattering)....... & $\mathrm{x}$ & $\mathrm{X}$ & $\mathrm{x}$ & & & $\mathrm{X}$ & $\mathrm{x}$ & $\mathrm{x}$ & $\mathrm{X}$ \\
\hline & 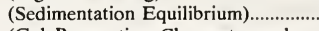 & $\mathrm{x}$ & $\mathrm{x}$ & & & $\mathrm{x}$ & & & & \\
\hline & (Gel Permeation Chromatog & & & $\mathrm{x}$ & & & & & & ........... \\
\hline & $\begin{array}{l}\text { GPC). } \\
\text { (Osmometry) }\end{array}$ & $\mathrm{X}$ & & & & $x$ & & $\mathrm{x}$ & $\mathrm{x}$ & \\
\hline Number Average ............................. & 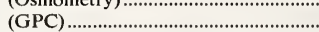 & & & \multirow{3}{*}{$\begin{array}{l}\mathrm{X} \\
\mathrm{X}\end{array}$} & & $\Lambda$ & & $\boldsymbol{A}$ & $\boldsymbol{A}$ & $\lambda$ \\
\hline Molecular Weight Distribution & (GPC) & & & & & & & & & ……... \\
\hline \multirow{2}{*}{$\begin{array}{l}\text { Limiting Viscosity Number } \\
\text { Toluene } 25^{\circ} \mathrm{C}\end{array}$} & (Capillary Viscometer)........ & & & & & $\mathrm{x}$ & & & & ............ \\
\hline & & & & & & & & & & ............ \\
\hline \multirow{2}{*}{$\begin{array}{l}\text { Benzene } 25^{\circ} \mathrm{C} \\
\text { Benzene } 35^{\circ} \mathrm{C}\end{array}$} & .................. & $\mathrm{x}$ & $\mathrm{x}$ & & & & & & ............. & …........ \\
\hline & & $\mathrm{x}$ & & & & & & & & …......... \\
\hline Cyclohexane $35^{\circ} \mathrm{C}$ & 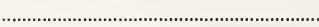 & $\mathrm{x}$ & $\mathrm{x}$ & & & & & & & \\
\hline \multirow{2}{*}{$\begin{array}{l}\text { 1-Chloronaphthalene } 130^{\circ} \mathrm{C} \ldots \ldots . \\
1,2,4 \text {-trichlorobenzene } 130^{\circ} \mathrm{C} \ldots\end{array}$} & & & & $\mathrm{x}$ & $\mathrm{x}$ & & 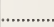 & $\mathrm{x}$ & $\mathrm{x}$ & $\mathrm{x}$ \\
\hline & $\ldots \ldots \ldots \ldots \ldots \ldots \ldots \ldots \ldots \ldots$ & & & $\mathrm{x}$ & $\mathrm{x}$ & & .......... & $\mathrm{x}$ & $\mathrm{x}$ & $\mathrm{X}$ \\
\hline Decahydronaphthalene $130^{\circ} \mathrm{C}$. & & & & $\mathrm{x}$ & $\mathrm{x}$ & & & & & \\
\hline \multirow{3}{*}{ Melt Flow } & $($ ASTM $) \ldots \ldots \ldots$ & & ............. & $\mathrm{x}$ & $\mathrm{x}$ & & & & .............. & $\ldots \ldots . . .$. \\
\hline & (ASTM) & & & $\mathrm{x}$ & $\mathrm{x}$ & & & & ............... & $\ldots \ldots . .$. \\
\hline & (Adiabatic) & $\mathrm{x}$ & ……...... & $\mathrm{x}$ & & & & & ............... & ........ \\
\hline
\end{tabular}

\section{Rheology Standard}

This SRM is intended for the calibration and checking of instruments used in polymer technology and science for the determination of rheological properties of polymer melts or solutions. It is certified for Rate of Shear, Viscosity, and First Normal Stress Difference at $25^{\circ} \mathrm{C}$.

\begin{tabular}{|c|c|c|}
\hline SRM & Type & Unit size \\
\hline 1490 & Polyisobutylene Solution in Cetane ............................... & $250 \mathrm{~mL}$ \\
\hline
\end{tabular}

\section{Heat Standards}

These SRM's are intended to relate heat and temperature measurements made in industrial, university, and government laboratories with the International Practical Temperature Scale-1968. ${ }^{1}$

\section{Superconductive Thermometric Fixed Point Devices}

Each device is composed of five small cylinders of high purity material mounted in a threaded copper stud and enclosed by a mutual inductance coil set. They should prove particularly valuable to users of ${ }^{3} \mathrm{He}-{ }^{4} \mathrm{He}$ dilution refrigerators, in which direct calibrations on the liquid helium vapor pressure-temperature scales are difficult, and to those who wish to determine the temperature reproducibility of physical phenomena or of cryogenic equipment.

\begin{tabular}{|c|c|c|c|}
\hline SRM & Type & Material & $\begin{array}{c}\text { Nominal } \\
\text { Temperature (K) }\end{array}$ \\
\hline $767 a$ & Superconductive Thermometric Fixed Point Device & $\begin{array}{l}\text { Lead } \\
\text { Indium } \\
\text { Aluminum } \\
\text { Zinc } \\
\text { Cadmium } \\
\text { Gold-Indium } \\
\text { Gold-Aluminum } \\
\text { Iridium } \\
\text { Beryllium } \\
\text { Tungsten }\end{array}$ & $\begin{array}{l}7.2 \\
3.4 \\
1.2 \\
0.8 \\
.5 \\
.205 \\
.157 \\
.098 \\
.024 \\
.015\end{array}$ \\
\hline
\end{tabular}

1 “International Practical Temperature Scale of 1968," Metrologia, 5, 535-44 (1969). 


\section{Defining Fixed Points-International Practical Temperature Scale}

These SRM's are of such purity that they are suitable for defining fixed points for the International Practical Temperature Scale of 1968.

\begin{tabular}{l|l|r|r}
\hline \multicolumn{1}{c|}{ SRM } & Type & Temperature ${ }^{\circ} \mathrm{C}$ & $\begin{array}{c}\text { Wt/Unit } \\
(\mathrm{grams})\end{array}$ \\
\hline 740 & Zinc & & \\
\hline 741 & Tin & & \\
\hline
\end{tabular}

\section{Determined Reference Points}

These SRM's are intended for use in calibration of thermometers, thermocouples, and other temperature measuring devices. The temperatures certified are in accord with the International Practical Temperature Scale of 1968.

\begin{tabular}{|c|c|c|c|}
\hline SRM & Type & Temperature ${ }^{\circ} \mathrm{C}$ & $\begin{array}{l}\text { Wt/Unit } \\
\text { (grams) }\end{array}$ \\
\hline $42 \mathrm{~g}$ & Tin & 231.967 & 350 \\
\hline $43 \mathrm{~h}$ & Zinc & *419.58 & 350 \\
\hline $44 \mathrm{f}$ & Aluminum & 660.3 & 200 \\
\hline $45 \mathrm{~d}$ & Copper & 1084.8 & 450 \\
\hline $49 \mathrm{e}$ & Lead & 327.493 & 600 \\
\hline 743 & Mercury & -38.841 & 680 \\
\hline
\end{tabular}

*SRM $43 \mathrm{~h}$ is less pure than SRM 740 and has a freezing point $0.001{ }^{\circ} \mathrm{C}$ lower.

Melting Point Standards

\begin{tabular}{|c|c|c|c|}
\hline SRM & Type & Temperature ${ }^{\circ} \mathrm{C}$ & $\begin{array}{l}\text { Wt/Unit } \\
\text { (grams) }\end{array}$ \\
\hline 742 & Alumina, $99.9+\%$ & 2053 & 10 \\
\hline 1968 & Gallium, 99.9999 + \% & 29.7723 & 25 \\
\hline
\end{tabular}

1 “International Practical Temperature Scale of 1968," Metrologia, 5, 535-44 (1969).

\section{Calorimetric Standards}

These SRM's are intended to relate the gain or loss of energy and work experienced during a chemical reaction or by change of temperature to the units of energy and work as defined by the National Measurement System. This system uses the units prescribed by the International System of Units (SI). The unit for energy and work under this system is the joule, which is related to the calorie by the equation: 4.184 joule $=1$ calorie.

\section{Combustion Calorimetric Standards}

\begin{tabular}{|c|c|c|}
\hline SRM & Type & Unit amount \\
\hline $39 \mathrm{i}$ & Benzoic acid, 26.4 absolute kilojoules/gram & $30 \mathrm{~g}$ \\
\hline $217 c-5$ & 2,2,4-Trimethylpentane & IN PREP \\
\hline
\end{tabular}




\begin{tabular}{|c|c|c|}
\hline SRM & Type & $\begin{array}{l}\text { Wt/Unit } \\
\text { (grams) }\end{array}$ \\
\hline $724 a$ & 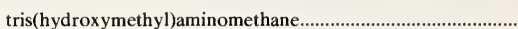 & 50 \\
\hline 1654 & 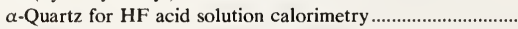 & 25 \\
\hline 1655 & Potassium Chloride & IN PREP \\
\hline
\end{tabular}

\section{Heat Source Calorimetric Standards}

\begin{tabular}{|c|c|c|}
\hline SRM & Type & $\begin{array}{l}\text { Wt/Unit } \\
\text { (grams) }\end{array}$ \\
\hline 1651 & Zirconium-barium chromate heat source powder $(\mathrm{ca} 350 \mathrm{cal} / \mathrm{g})$ & 50 \\
\hline 1652 & Zirconium-barium chromate heat source powder $(\mathrm{ca} 390 \mathrm{cal} / \mathrm{g})$ & 50 \\
\hline 1653 & Zirconium-barium chromate heat source powder $(\mathrm{ca} 425 \mathrm{cal} / \mathrm{g})$ & 50 \\
\hline
\end{tabular}

\section{Enthalpy and Heat Capacity Standards}

\begin{tabular}{|c|c|c|c|}
\hline SRM & Type & Range $\mathbf{K}$ & Unit Size \\
\hline 705 & Polystyrene, powder r. & $10-350$ & $5 g$. \\
\hline 720 & Sapphire, synthetic $\left(\mathrm{Al}_{2} \mathrm{O}_{3}\right)$ & $0-2250$ & $15 \mathrm{~g}$. \\
\hline 729 & Graphite, rod & $300-2500$ & $10 \mathrm{~cm} \times 0.64 \mathrm{~cm} \mathrm{D}$. \\
\hline 781-D1 & Molybdenum, sintered rod & $273.15-2800$ & $10 \mathrm{~cm} \times 0.32 \mathrm{~cm} \mathrm{D}$. \\
\hline 781-D2 & Molybdenum, sintered rod & $273.15-2800$ & $10 \mathrm{~cm} \times 0.64 \mathrm{~cm} \mathrm{D}$. \\
\hline 782 & Tungsten, rod & $273-1200$ & $10 \mathrm{~cm} \times 0.32 \mathrm{~cm} \mathrm{D}$. \\
\hline 1475 & 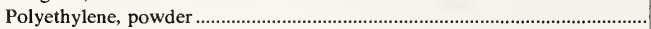 & $5-360$ & $50 \mathrm{~g}$ \\
\hline
\end{tabular}

\section{Vapor Pressure Standards}

These SRM's are intended for use in the testing and calibration of vapor pressure measurement apparatus and techniques.

\begin{tabular}{|c|c|c|c|c|}
\hline SRM & Type & $\begin{array}{l}\text { Pressure Range } \\
\text { (atmosphere) }\end{array}$ & $\begin{array}{l}\text { Tempera- } \\
\text { ture Range } \\
(\mathbf{K})\end{array}$ & Unit Size \\
\hline 745 & 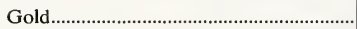 & $10^{-3}$ to $10^{-8}$ & $1300-2100$ & Wire $1.44 \mathrm{~mm} \times 152 \mathrm{~mm}$. \\
\hline 746 & Cadmium & $10^{-4}$ to $10^{-11}$ & $350-594$ & Rod $6.4 \mathrm{~mm} \times 64 \mathrm{~mm}$ \\
\hline 748 & Silver & $10^{-3}$ to $10^{-12}$ & $800-1600$ & Rod $6.4 \mathrm{~mm} \times 64 \mathrm{~mm}$ \\
\hline
\end{tabular}

\section{Conductivity Standards}

This SRM is intended for use in calibrating eddy current devices for measuring the electrical conductivity of metals.

\begin{tabular}{|c|c|c|}
\hline SRM & Type & Conductivity, \% IACS \\
\hline 1456 & Set of 4 aluminum alloy blocks, $4.5 \times 4.5 \times 1 \mathrm{~cm}$ & $29,41,48$ and 60 \\
\hline
\end{tabular}


These SRM's are intended for use calibrating instruments used to measure the electrical resistivity of silicon wafers using both four-probe and eddy current methods.

\begin{tabular}{|c|c|c|}
\hline SRM & Type & Unit of Issue \\
\hline 1521 & Boron Doped Silicon, 0.1 and $10 \Omega \cdot \mathrm{cm}$ & Set of 2 wafers \\
\hline 1522 & 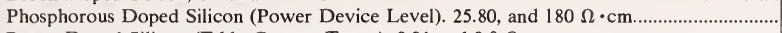 & Set of 3 wafers \\
\hline 1523 & 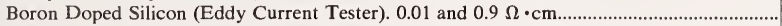 & Set of 2 wafers \\
\hline
\end{tabular}

\section{Thermal Conductivity and Electrical Resistance Standards}

These SRM's cover the high, medium, and low conductivity ranges. They will be useful for intercomparing thermal conductivity apparatus, debugging new apparatus, and calibrating comparative apparatus.

\begin{tabular}{|c|c|c|c|c|}
\hline SRM & Type & Temperature Range (K) & Diameter (mm) & Length (mm) \\
\hline 1465 & Tungsten, sintered & $4-3000$ & 3.2 & 50 \\
\hline 1466 & Tungsten, sintered & $4-3000$ & 6.4 & 50 \\
\hline 1467 & Tungsten, arc-cast & $4-3000$ & 8.3 & 50 \\
\hline 1468 & 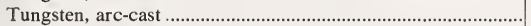 & 4-3000 & 10.2 & 50 \\
\hline 1469 & Tungsten, arc-cast & $4-3000$ & 12.7 & 50 \\
\hline 1463 & 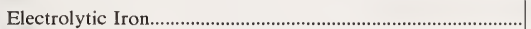 & $4-1000$ & 6.4 & 305 \\
\hline 1464 & 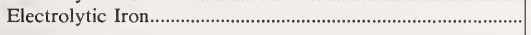 & $4-1000$ & 31.8 & 152 \\
\hline 1460 & Stainless Steel & $5-1200$ & 6.4 & 50 \\
\hline 1461 & 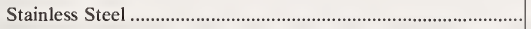 & $5-1200$ & 12.7 & 50 \\
\hline 1462 & Stainless Steel & $5-1200$ & 34 & 50 \\
\hline
\end{tabular}

\section{Thermal Expansion Standards}

These SRM's cover the temperature range from 20 to $2000 \mathrm{~K}$ having coefficients of thermal expansion over the range of 0.5 to $17 \times 10^{-6} \mathrm{~K}$.

\begin{tabular}{|c|c|c|c|c|}
\hline SRM & Type & Temperature Range (K) & Diameter (mm) & Length (mm) \\
\hline 731-L1 & 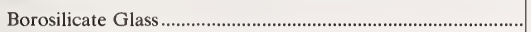 & $80-680$ & 6.4 & 51 \\
\hline $731-\mathrm{L} 2$ & 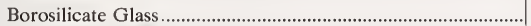 & $80-680$ & 6.4 & 102 \\
\hline $731-\mathrm{L} 3$ & 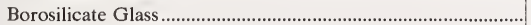 & $80-680$ & 6.4 & 152 \\
\hline 732 & 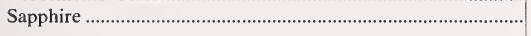 & $293-2000$ & 6.4 & 51 \\
\hline $736 a$ & Copper (IN PREP) & $20-800$ & 6.4 & 51 \\
\hline 737 & Tungsten & $80-1800$ & 6.4 & 51 \\
\hline 738 & Stainless Steel & $293-900$ & 6.4 & 51 \\
\hline 739-Ll & Fused Silica. & $80-1000$ & 6.4 & 51 \\
\hline 739-L2 & Fused Silica. & $80-1000$ & 6.4 & 102 \\
\hline 739-L3 & 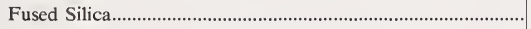 & $80-1000$ & 6.4 & 152 \\
\hline
\end{tabular}


These SRM's are intended to serve as a convenient mechanism for the comparison of manufactured wire to standard reference thermocouple tables.

\begin{tabular}{|c|c|c|}
\hline SRM & Type & Form \\
\hline $\begin{array}{r}733 \\
1967\end{array}$ & 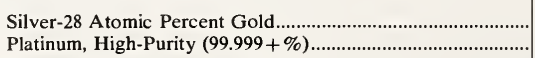 & $\begin{array}{l}\text { Wire: } 32 \mathrm{AWG}(0.2019 \mathrm{~mm}) \text { diameter, } 3 \text { meters long. } \\
\text { Wire: } 0.51 \mathrm{~mm} \text { diameter, } 1 \text { meter long. }\end{array}$ \\
\hline
\end{tabular}

\section{Thermal Resistance Standards}

This SRM is intended for use in calibrating, and verifying apparatus such as the guarded hot-plate (ASTM C177) and heat flow meter (ASTM C518) used to determine the thermal resistance of thermal insulation materials.

\begin{tabular}{|c|c|c|c|c|}
\hline SRM & Type & Density & Temperature & Size \\
\hline $1450 \mathrm{~b}$ & Fibrous Glass Board (IN PREP) & & & \\
\hline 1451 & 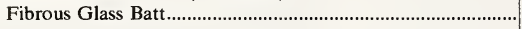 & $\sim 13$ & $255-330$ & $60 \times 60 \times 2.54$ \\
\hline
\end{tabular}

${ }^{*}$ Smaller square can be supplied.

\section{Magnetic Standards}

\section{Magnetic Susceptibility Standards}

These SRM's are intended for use in the calibration of instruments used to measure magnetic susceptibility.

\begin{tabular}{|c|c|c|}
\hline SRM & Type & Form/Unit \\
\hline $763-1$ & Aluminum ........................................ & Cylinder $3 \mathrm{~mm}$ diameter $\times 3 \mathrm{~mm}$. \\
\hline $763-2$ & Aluminum & Wire $0.5 \mathrm{~mm}$ diameter $\times 250 \mathrm{~mm}$. \\
\hline $763-3$ & Aluminum & Rod $6 \mathrm{~mm}$ diameter $\times 175 \mathrm{~mm}$. \\
\hline $764-1$ & 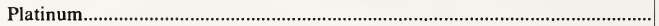 & Cylinder $3 \mathrm{~mm}$ diameter $\times 3 \mathrm{~mm}$. \\
\hline $764-2$ & 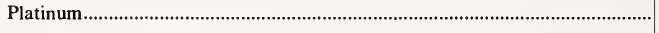 & Wire $0.5 \mathrm{~mm}$ diameter $\times 50 \mathrm{~mm}$. \\
\hline $765-1$ & Palladium & Cylinder $3 \mathrm{~mm}$ diameter $\times 3 \mathrm{~mm}$. \\
\hline $765-2$ & 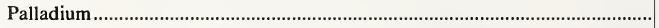 & Wire $0.5 \mathrm{~mm}$ diameter $\times 50 \mathrm{~mm}$. \\
\hline $765-3$ & Palladium & Sponge 1 gram \\
\hline $766-1$ & Manganese Fluoride & Cube $3 \times 3 \times 3 \mathrm{~mm}$ \\
\hline
\end{tabular}

\section{Magnetic Moment}

This SRM is intended for the calibration of instruments used to measure magnetic moment.

\begin{tabular}{|c|c|c|}
\hline SRM & Type & Size \\
\hline 772 & Nickel Sphere & $2.4 \mathrm{~mm} \mathrm{D}$. \\
\hline
\end{tabular}


This SRM is intended for use in electron paramagnetic resonance (EPR) measurements for determining the number of active paramagnetic centers in a test sample. This SRM consists of two pieces of synthetic ruby.

\begin{tabular}{|c|c|c|}
\hline SRM & Type & Form \\
\hline 2601 & Crystalline $\mathrm{Al}_{2} \mathrm{O}_{3} ; \mathrm{Cr}^{3+}$ (Ruby) & $\begin{array}{l}1.5 \times 1.5 \times 0.5 \mathrm{~mm} . \\
0.5 \times 0.5 \times 4 \mathrm{~mm} .\end{array}$ \\
\hline
\end{tabular}

\section{Optical Standards}

\section{Spectrophotometric Standards}

\section{Filters for Spectrophotometry and Luminescence}

The spectrophotometric SRM's are intended primarily for use in verifying the accuracy of the transmittance scale of spectrophotometers. The luminescence SRM provides relative emission spectra to determine spectral responsivity and to verify the accuracy of spectrofluorimeters. All of these SRM's provide a means of interlaboratory comparison of data.

Glass Filters, SRM 930D, consists of three neutral glass filters. The glass filters have transmittances of approximately 10, 20, and 30 percent. Each filter is individually calibrated and certified for transmittance and transmission density at wavelengths of 440,465, 546.1, 590, and $635 \mathrm{~nm}$. The $546.1 \mathrm{~nm}$ wavelength coincides with the Mercury emission line.

Liquid Filters, SRM 931b, are absorbance standards for use in ultraviolet and visible spectrophotometry. This SRM consists of 3 sets of 4 vials, each containing a blank solution and three solutions of different concentrations of an absorbing liquid. Each vial contains approximately $10 \mathrm{~mL}$ of solution. The net absorbances are certified for each concentration at wavelengths $302,395,512$, and $678 \mathrm{~nm}$.

Quartz Cuvette, SRM 932, is an all-quartz rectangulr parallelepiped cuvette designed to fit the holder of conventional spectrophotometers. The distances between the parallel, optically-transparent windows are measured at 10 positions along the vertical axis. The cuvettes range in pathlength between 9.97 and 10.03 $\mathrm{mm}$, and the inner surfaces of the opposite windows are parallel within $\pm 0.002 \mathrm{~mm}$. Each cuvette is certified for pathlength and parallelism of the windows to within $\pm 0.0005 \mathrm{~mm}$.

Potassium Dichromate, SRM 935, consists of crystalline potassium dichromate of established purity certified for use as an ultraviolet absorbance standard. Solutions made with this SRM in $0.001 \mathrm{~N}$ perchloric acid are certified for their apparent specific absorbances, $\epsilon_{\mathrm{a}}$, at $23.5^{\circ} \mathrm{C}$ and wavelengths of $235,257,313$, 345 , and $350 \mathrm{~nm}$.

Quinine Sulfate Dihydrate, SRM 936, consists of powdered quinine sulfate dihydrate of known purity certified for use as a spectrofluorimetric emission standard. A solution made with this SRM in $0.1 \mathrm{~N}$ perchloric acid is certified for its molecular emission spectrum, $\mathrm{E}(\lambda)$ at $25.0^{\circ} \mathrm{C}$ over the wavelength range of 375.0 to $675 \mathrm{~nm}$.

Didymium-Oxide Glass Filters, SRM's 2009, 2010, 2013 and 2014, are wavelength standards for use in checking the wavelength scale of spectrophotometers between 400 and $760 \mathrm{~nm}$ for bandpasses between 1.5 and $10.5 \mathrm{~nm}$. SRM's 2009 and 2010 were batch calibrated whereas SRM's 2013 and 2014 were individually calibrated. SRM's 2009 and 2013 are approximately $1 \mathrm{~cm}$ wide by $3 \mathrm{~cm}$ high and are supplied in a holder which fits in the place of a standard analytical cuvette. SRM's 2010 and 2014 are in the form of squares approximately $5.1 \mathrm{~cm}$ by $5.1 \mathrm{~cm}$. 
Glass Filter, SRM 2030, consists of one neutral glass filter. It is intended as a reference source for onepoint verification of the transmittance and absorbance scales of spectrophotometers at a wavelength of 465 $\mathrm{nm}$ and a nominal $30 \%$ transmittance.

Metal-on-Quartz Filters, SRM 2031, consists of three filters mounted in metal holders and an empty holder, all holders are equipped with shutters. Two of the filters have an evaporated layer of semitransparent metal sandwiched between two quartz plates that have been assembled by optical contact. The third filter consists of two clear quartz plates assembled by the same technique. Each filter is individually calibrated at $250,280,340,360,400,465,500,546,590$ and $635 \mathrm{~nm}$.

Potassium Iodide, SRM 2032, consists of crystalline KI of established purity for use as a stray light standard in the ultraviolet. Aqueous solutions made with this material are certified for their specific absorbance at $23.5^{\circ} \mathrm{C}$ over a wavelength range from 240 to $280 \mathrm{~nm}$.

Potassium Iodide with Attenuator, SRM 2033, consists of the same material used for SRM 2032 plus a reference beam attenuator for extending the dynamic range of the stray light test.

\begin{tabular}{|c|c|c|}
\hline SRM & Type & Unit \\
\hline 930D & Glass Filters for Spectrophotometry ..... & Set: 3 filters, 4 holders \\
\hline $931 \mathrm{c}$ & Liquid Filters for Spectrophotometry & Set: 12 vials \\
\hline 932 & Quartz Cuvette for Spectrophotometry & 1 each \\
\hline 935 & 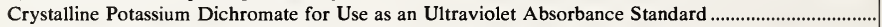 & 15 grams \\
\hline 936 & Quinine Sulfate Dihydrate & 1 gram \\
\hline 2009 & Didymium-oxide glass & 1 filter in holder \\
\hline 2010 & Didymium-oxide glass & $51 \times 51 \mathrm{~mm}$ \\
\hline 2013 & Didymium-oxide glass & 1 filter in holder \\
\hline 2014 & Didymium-oxide glass & $51 \times 51 \mathrm{~mm}$ \\
\hline 2030 & Glass Filter for Transmittance Measurement & 1 filter, 2 holders \\
\hline 2031 & Metal-on-Quartz Filters for Spectrophotometry & \\
\hline 2032 & Potassium Iodide for Use as a Stray Light Standard & \\
\hline 2033 & Potassium Iodide with Attenuator for Use as Stray Light Standard & IN PREP \\
\hline
\end{tabular}

\section{Reflectance Standards}

These SRM's are intended primarily for calibration of the reflectance scale of integrating sphere reflectometers used in evaluating materials for solar energy collectors and for calibration of reflectometers used in evaluating the appearance of polished metals and metal plated objects.

\section{Specular Spectral Reflectance Standards}

SRM 2003a is a first-surface mirror of vacuum deposited aluminum on glass that is certified for nearnormal reflectance over the wavelenth range of 250 to $2500 \mathrm{~nm}$. SRM's 2023, 2024 and 2025 are secondsurface mirrors of vacuum deposited aluminum on a fused quartz plate and covered with a second plate of fused quartz. These mirrors are also certified for near-normal reflectance from 250 to $2500 \mathrm{~nm}$ and for a few wavelengths at 15, 30, 45 and 60 degrees from normal. SRM 2025 has a small angle between the front and rear surfaces.

\begin{tabular}{|c|c|c|}
\hline SRM & Type & Size \\
\hline 2003a & First Surface, Aluminum on Glass...................... & $5.1 \mathrm{~cm}$ dia. \\
\hline 2023 & Second Surface, Aluminum on Fused Quartz. & $5.1 \times 5.1 \mathrm{~cm}$ \\
\hline 2024 & Second Surface, Aluminum on Fused Quartz & $2.5 \times 10.2 \mathrm{~cm}$ \\
\hline 2025 & Second Surface, Aluminum on Fused Quartz & $2.5 \times 10.2 \mathrm{~cm}$ \\
\hline
\end{tabular}


SRM's 2015 and 2016 are made from a white glass and are certified for near-normal reflectance from 400 to $750 \mathrm{~nm}$. SRM's 2019 and 2020 are white ceramic tiles certified from 350 to $2500 \mathrm{~nm}$. SRM's 2021 and 2022 are black porcelain enamel squares certified from 280 to $2500 \mathrm{~nm}$. These last four SRM's are primarily certified for near-nomal reflectance but are also certified for reflectance at a few wavelengths at $15,30,45$ and 60 degrees from normal.

\begin{tabular}{|c|c|c|}
\hline SRM & Type & Size \\
\hline 2015 & White Glass... & $2.5 \times 5.0 \times 0.64 \mathrm{~cm}$ \\
\hline 2016 & White Glass................... & $10 \times 10 \times 0.64 \mathrm{~cm}$ \\
\hline 2019 & White Ceramic Tile & $5.1 \times 5.1 \times 0.81 \mathrm{~cm}$ \\
\hline 2020 & White Ceramic Tile & $3.8 \times 7.6 \times 0.81 \mathrm{~cm}$ \\
\hline 2021 & Black Porcelain Enamel. & $5.1 \times 5.1 \times 0.20 \mathrm{~cm}$ \\
\hline 2022 & 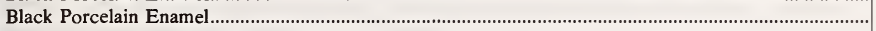 & $2.5 \times 2.5 \times 0.20 \mathrm{~cm}$ \\
\hline
\end{tabular}

\section{Refractive Index Standards}

SRM's $211 \mathrm{c}$ and $217 \mathrm{c}$ are certified for refractive index at 20,25 and $30^{\circ} \mathrm{C}$, from 435.8 to $667.8 \mathrm{~nm}$ for seven wavelengths, and are available in 5 and $25 \mathrm{~mL}$ ampoules.

SRM's 1820 and 1822 are certified for refractive index at thirteen wavelengths from $404.7 \mathrm{~nm}$ to 706.5 $\mathrm{nm}$. These SRM's are designed for calibrating refractometers and certifying refractive index immersion liquids, and should provide a basis for accurate measurements of refractive index and dispersion. They consist of two rectangular glass slabs: one slab has polished faces and is to be used to check the performance of a refractometer; the second slab is unpolished and can be broken into fragments to certify the refractive index of immersion liquids by microscopic methods.

SRM 1823 consists of two silicone liquids that are chemically and thermally stable. The liquids are miscible and span the refractive index range of a variety of glasses and glass fibers that are examined microscopically by immersion techniques. Used independently, the liquids are suitable for the calibration of refractometers. These liquids are certified for refractive index at ten wavelengths from 435.8 to $667.8 \mathrm{~nm}$, at temperatures of $20,40,60$ and $80^{\circ} \mathrm{C}$.

\begin{tabular}{|c|c|c|}
\hline SRM & Type & $\mathrm{n}^{20}$ \\
\hline $211 \mathrm{c}$ & ㄴ. & 1.497 \\
\hline $217 \mathrm{c}$ & 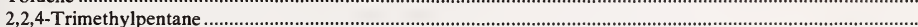 & 1.391 \\
\hline 1820 & 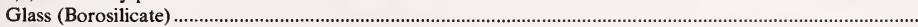 & 1.488 \\
\hline 1822 & Glass (Soda-Lime) & 1.518 \\
\hline $1823-\mathrm{I}$ & Silicone Liquid (I) & 1.518 \\
\hline 1823-II & Silicone Liquid (II) & 1.559 \\
\hline
\end{tabular}

\section{Radioactivity Standards}

Information concerning the SRM appears on it or its container. A Certificate containing pertinent information on the SRM is sent under separate cover; a photocopy of the certificate is sent with the SRM. Copies of these Certificates and information concerning the applications of these SRM's are available on request to the NBS Office of Standard Reference Materials. These materials are shipped only by express or air freight (shipping charges collect). The prices of SRM's may change as current stocks are depleted and are replaced. Purchasers will be billed at the prices in effect at the time of shipment. 
The stated accuracies of the older standards are, in general, an estimate of the standard deviation added to an estimate of maximum possible systematic error. The accuracies of more recent standards are based on the 99 percent confidence level of precision, with the same estimate of systematic error.

The International Commission on Radiation Units (ICRU) recommended definition of the activity (A) of a quantity of a radioactive nuclide is the quotient of $\Delta \mathrm{N}$ by $\Delta \mathrm{t}$, where $\Delta \mathrm{N}$ is the number of nuclear transformations that occur in this quantity, in time $\Delta \mathrm{t}$ : $(\mathrm{A}=\Delta \mathrm{N} / \Delta \mathrm{t})$. NBS has used the abbreviation ntps for nuclear transfromation per second. In this list both ntps and dps are used: the latter when dps has been used in certificates printed before $1968 .{ }^{2}$ The terms: $\alpha \mathrm{ps}, \beta^{-} \mathrm{ps}, \beta^{+} \mathrm{ps}, \mathrm{K}$-x-rays ps, $\gamma \mathrm{ps}$ are used for the emission rates of alpha particles, beta particles, positrons, $\mathrm{K}-\mathrm{x}$-rays, and gamma-rays, respectively.

The SRM's listed below, not marked with an asterisk(*), may be ordered singly, without a license, under the general licensing provisions of the Atomic Energy Act of 1954. Those marked by an asterisk are available only under the special licensing provisions of the Atomic Energy Act of 1954 .

NOTE: Certain radionuclides are not economical to maintain in stock because of short half lives or low demand. When sufficient demand exists, based on letters of inquiry, these materials are prepared and those who have expressed interest are notified of their availability. If you need any radionuclides not listed, contact the Radioactivity Section, Room C114, Radiation Physics Building, National Bureau of Standards, Washington, D.C. 20234 (Telephone: 301-921-2668).

In addition, chemically stable solutions of most radionuclides, including those no longer issued by NBS or that are currently out of stock, may be submitted to NBS for calibration as described in "Calibration and Related Measurement Services of the National Bureau of Standards," NBS Special Publication 250 (1980). Requests for these tests should be submitted, with full source information for approval of suitability, to the Radioactivity Section.

Alpha-Particle, Beta-Particle, Gamma-Ray, and Electron-Capture Solution Standards

\begin{tabular}{|c|c|c|c|c|c|}
\hline SRM & Radionuclide & \multicolumn{2}{|c|}{$\begin{array}{l}\text { Approximate activity, per gram, at time of } \\
\text { calibration (month/year) }\end{array}$} & $\begin{array}{l}\text { Approx. } \\
\text { mass of } \\
\text { solution }\end{array}$ & $\begin{array}{c}\text { Overall } \\
\text { uncertainty }\end{array}$ \\
\hline 4229 & Alumimum-26 & 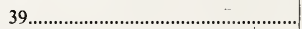 & $11 / 71$ & 4.6 & \pm 1.1 \\
\hline $4219 B$ & Cadmium-109 & $10^{5}$ & $11 / 76$ & 5 & 2.0 \\
\hline 4245 & 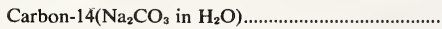 & 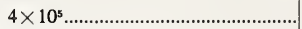 & $5 / 74$ & 5 & 1.0 \\
\hline 4246 & 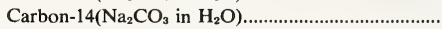 & 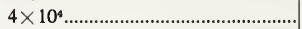 & $5 / 74$ & 5 & 0.9 \\
\hline 4247 & 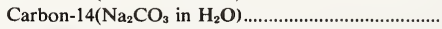 & $4 \times 10^{2}$ & $5 / 74$ & 5 & 1.1 \\
\hline 4925 & Carbon-14 $\left(\mathrm{C}_{6} \mathrm{H}_{5} \mathrm{COOH}\right.$ in $\left.\mathrm{C}_{6} \mathrm{H}_{5} \mathrm{CH}_{3}\right) \ldots \ldots \ldots \ldots \ldots \ldots$ & 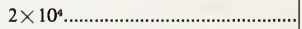 & $7 / 58$ & 3 & 2.4 \\
\hline 4222 & Carbon-14($\left(\mathrm{C}_{16} \mathrm{H}_{34}\right)$ & 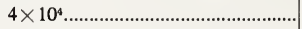 & $6 / 67$ & 3 & 3.1 \\
\hline 4223 & 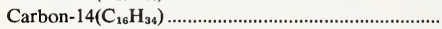 & 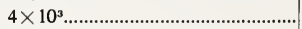 & $6 / 67$ & 3 & 3.1 \\
\hline 4224 & Carbon-14( $\left.\mathrm{C}_{16} \mathrm{H}_{34}\right)$ & 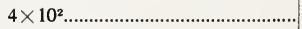 & $6 / 67$ & 3 & 3.1 \\
\hline $4233 B^{*}$ & Cesium-137 & 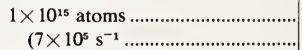 & $8 / 79$ & 5.1 & $\begin{array}{l}0.5 \\
1.4\end{array}$ \\
\hline 4943 & 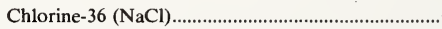 & 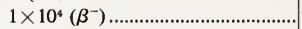 & 1962 & 3 & 2.3 \\
\hline $4422 L^{*}$ & Chlorine-36 (HC1) & 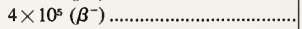 & 1980 & 5.1 & 1.6 \\
\hline 4913B & Cobalt-60 & 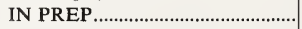 & ................ & ..................... & .................... \\
\hline $4370 B^{*}$ & Europium-152 & 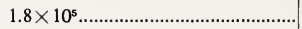 & $6 / 79$ & 5 & 1.5 \\
\hline $4926 \mathrm{C}$ & Hydrogen-3 $\left(\mathrm{H}_{2} \mathrm{O}\right)$ & $3 \times 10^{3} \ldots \ldots \ldots \ldots \ldots \ldots \ldots \ldots \ldots \ldots$ & $9 / 78$ & 18 & 0.6 \\
\hline 4927B & Hydrogen-3 $\left(\mathrm{H}_{2} \mathrm{O}\right)$ & $78 \times 10^{5} \ldots \ldots \ldots \ldots \ldots$ & $9 / 78$ & 3 & 0.6 \\
\hline 4947 & Hydrogen-3 $\left(\mathrm{C}_{6} \mathrm{H}_{5} \mathrm{CH}_{3}\right)$ & 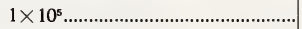 & $9 / 78$ & 4 & 1.0 \\
\hline $4929 \mathrm{C}$ & Iron-55 & $2 \times 10^{4}(\mathrm{k}-\mathrm{x})$ & $4 / 70$ & 3.9 & 2.7 \\
\hline $4257^{*}$ & 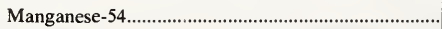 & 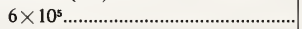 & $4 / 79$ & 5 & 1.02 \\
\hline 4226 & Nickel-63 & 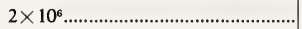 & $5 / 68$ & 4.1 & 1.0 \\
\hline 4331 & Plutonium-239 & $6(\alpha)$ & $3 / 75$ & 2 & 1.0 \\
\hline 4940B & Promethium-147 & 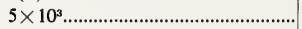 & $2 / 76$ & 3 & 1.9 \\
\hline 4919D & Strontium-Yttrium-90 & 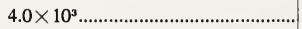 & $4 / 75$ & 2.9 & 2.1 \\
\hline
\end{tabular}

${ }^{2}$ NBS now uses the SI (International System of Units) unit for activity, which is "1 per second," symbolized as s ${ }^{-1}$. 
These SRM's consist of a practically weightless deposit of the nuclide on a thin platinum foil cemented to a monel disk.

\begin{tabular}{|c|c|c|c|c|}
\hline SRM & Radionuclide & $\begin{array}{l}\text { Approx. } \alpha \text {-particle-emission rate i } \\
\text { geometry and/or approx. activity } \\
\text { of calibration (month/year) }\end{array}$ & $\begin{array}{l}\text { to } 2 \pi \\
\text { time }\end{array}$ & $\begin{array}{l}\text { Overall uncertainty } \\
(\%)\end{array}$ \\
\hline $\begin{array}{l}4904 \mathrm{~F} \\
4907 \\
4906 \mathrm{~B}\end{array}$ & 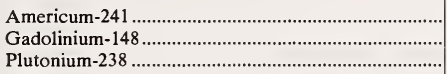 & 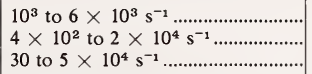 & $\begin{array}{l}9 / 77 \\
8 / 79 \\
7 / 78\end{array}$ & $\begin{array}{r} \pm 1.3 \\
0.7 \text { to } 1.6 \\
0.7 \text { to } 2.2\end{array}$ \\
\hline
\end{tabular}

Contemporary Standard for Carbon-14 Dating Labarataries

\begin{tabular}{c|c|c}
\hline \multicolumn{1}{c|}{ SRM } & Material & \multicolumn{1}{c}{ Description } \\
\hline RM49 & Oxalic Acid …………………………….......... & $\begin{array}{l}\text { One-half pound of oxalic acid taken from specially prepared material } \\
\text { for use as a common contemporary standard against which world- } \\
\text { wide measurements can be compared; no specific activity given; no } \\
\text { certificate issued. }\end{array}$ \\
\hline
\end{tabular}

Beta-Particle and Gamma-Ray Gas Standards

These SRM's contain the radionuclide in the inactive gas at a pressure of about one atmosphere in a glass break-seal ampoule.

\begin{tabular}{|c|c|c|c|c|c|}
\hline SRM & Radionuclide & \multicolumn{2}{|c|}{$\begin{array}{l}\text { Approximate activity, radioactivity } \\
\text { concentration or emission rate at time of } \\
\text { calibration (month/year) }\end{array}$} & $\begin{array}{l}\text { Approximate } \\
\text { volume } \\
(\mathrm{mL})\end{array}$ & $\begin{array}{l}\text { Overall } \\
\text { uncertainty } \\
(\%)\end{array}$ \\
\hline 4302 & Argon-39 & 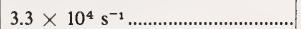 & $7 / 75$ & 10 & \pm 1.5 \\
\hline $4935 \mathrm{C}$ & Krypton-85 & 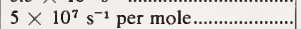 & $3 / 74$ & 10 & 0.9 \\
\hline 4235 & Krypton-85 & $9 \times 10^{6}$ to $1.5 \times 10 \mathrm{~s}^{-1} \ldots \ldots \ldots \ldots$. & $11 / 74$ & 3 & 1.2 \\
\hline $4308 B$ & Krypton-85 & 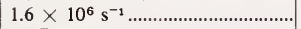 & $11 / 79$ & 32.5 & 3.1 \\
\hline $4309 \mathrm{C}$ & Xenon-127 & IN PREP & & & \\
\hline
\end{tabular}

\section{Gamma-Ray "Point-Saurce" Standards}

This group of Standard Reference Materials is usually prepared by depositing the radioactive material and sealing it between two layers of polyester tape, mounted on an aluminum ring. Exceptions to this procedure are americium, krypton, and thorium SRM's. The americium-241 SRM's, 4211 and 4213, are prepared by electroplating americium onto a $0.010-\mathrm{cm}$ thick platinum foil, which is covered with a $0.005-\mathrm{cm}$ thick aluminum foil. The aluminum-covered source is sandwiched between two layers of $0.036-\mathrm{cm}$ thick polyurethane film tape. The krypton- 85 SRM, 4212, is prepared by sealing a krypton- 85 impregnated aluminum foil between two glass disks, with an epoxy adhesive. The thorium-228 SRM, 4206 (In Prep), is prepared by depositing and sealing the radionuclide between two layers of gold foil and this sandwich is then sealed between two double layers of polyurethane-film tape. 


\begin{tabular}{|c|c|c|c|c|}
\hline SRM & Radionuclide & $\begin{array}{l}\text { Gamma-ray } \\
\text { energy }(\mathrm{MeV})\end{array}$ & $\begin{array}{c}\text { Approximate activity, } \mathrm{s}^{-1} \text {, at time of } \\
\text { calibration (except MRN) (month/ } \\
\text { year) }\end{array}$ & $\begin{array}{l}\text { Overall } \\
\text { uncertainty } \\
(\%)\end{array}$ \\
\hline 4211 & Americium-241 & 0.060 & 4.0 to $18 \times 10^{4}-2 / 70 \ldots \ldots \ldots \ldots \ldots \ldots \ldots \ldots$ & \pm 2.8 \\
\hline 4213 & 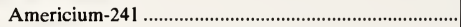 & 0.060 & 1.9 to $4.1 \times 10^{4}-2 / 70 \ldots \ldots \ldots \ldots \ldots \ldots \ldots \ldots$ & 2.8 \\
\hline $4202 \mathrm{C}$ & 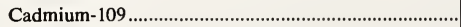 & 0.088 & 3 to $6 \times 10^{3}-11 / 76 \ldots \ldots \ldots$. & 2.1 \\
\hline 4212 & 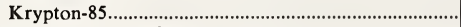 & 0.514 & 6.5 to $36.5 \times 10^{6}-5 / 71 \ldots \ldots \ldots \ldots \ldots \ldots \ldots$ & 2.6 \\
\hline $4200 \mathrm{~B}$ & 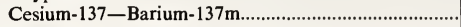 & 0.662 & 2.6 to $6.2 \times 10^{4}-9 / 79 \ldots \ldots \ldots \ldots \ldots \ldots \ldots \ldots$ & 1.6 \\
\hline
\end{tabular}

Gamma-Ray "Point-Source" Standards

These SRM's are listed in order of ascending gamma-ray energy (except the ${ }^{152} \mathrm{Eu}$ ).

\begin{tabular}{|c|c|c|c|c|}
\hline SRM & Radionuclide & $\begin{array}{c}\text { Gamma-ray energy } \\
(\mathrm{MeV})\end{array}$ & $\begin{array}{l}\text { Approximate activity, } \mathrm{s}^{-1} \text {, at time } \\
\text { of calibration (except MRN) } \\
\text { (month/year) }\end{array}$ & $\begin{array}{c}\text { Overall } \\
\text { uncertainty } \\
(\%)\end{array}$ \\
\hline 4207 & 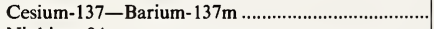 & 0.662 & 1.7 to $3.6 \times 10^{5}-9 / 79 \ldots \ldots \ldots \ldots \ldots \ldots$ & \pm 1.6 \\
\hline 4201B & 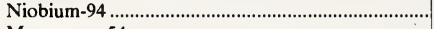 & $0.702,0.871$ & 4 to $6 \times 10^{3}-4 / 70 \ldots \ldots \ldots \ldots \ldots \ldots \ldots \ldots \ldots \ldots$ & 1.5 \\
\hline *4997E & 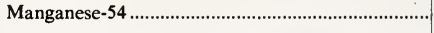 & 0.835 & 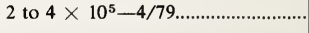 & 1.02 \\
\hline 4240 & 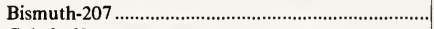 & $0.5696,1.0634,1.7697$ & 5.4 to $11 \times 10^{4}-1 / 73 \ldots \ldots \ldots \ldots \ldots$ & 1.4 \\
\hline $4203 \mathrm{C}$ & 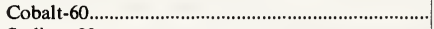 & $1.173,1.333$ & 1.0 to $2.3 \times 10^{5}-2 / 73 \ldots \ldots \ldots \ldots \ldots \ldots$ & 1.2 \\
\hline $4991 C$ & 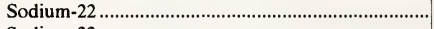 & 1.2745 & 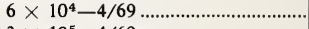 & 1.5 \\
\hline 4996B & Sodium-22 & 1.2745 & $3 \times 10^{5}-4 / 69$ & 1.5 \\
\hline *4218D & Europium-152 & $0.122-1.40$ & $4 \times 10^{4}$ to $5 \times 10^{5}-6 / 79 \ldots \ldots \ldots$ & 1.5 \\
\hline
\end{tabular}

\section{Low Energy Photon Sources}

These SRM's consist of a thin-layer deposit of the radionuclide on a thin stainless steel or platinum foil cemented to a monel disk.

\begin{tabular}{|c|c|c|c|}
\hline SRM & Radionuclide & $\begin{array}{l}\text { Approx. emission rate at time of calibration } \\
\text { (month/year) }\end{array}$ & $\begin{array}{c}\text { Overall } \\
\text { uncertainty } \\
(\%)\end{array}$ \\
\hline $\begin{array}{l}4262 \\
4260 \mathrm{~B}\end{array}$ & Americium-241 & 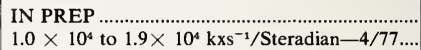 & \pm 1.8 \\
\hline
\end{tabular}

\section{Radium Solution Standards for Radon Analysis}

These samples are contained in flame-sealed glass ampoules.

\begin{tabular}{|c|c|c|c|}
\hline SRM & Nominal Radium Content. Per Gram (Month/Year) & $\begin{array}{l}\text { Approx. } \\
\text { Wt. Soln. } \\
\text { (grams) }\end{array}$ & $\begin{array}{c}\text { Uncertainty } \\
(\%)\end{array}$ \\
\hline 4952B & Blank Solution $-8 / 76$ & 20 & \pm 68 \\
\hline $4951 \mathrm{C}$ & $10^{-11}-4 / 78$ & 10.4 & 1.8 \\
\hline 4950D & $10^{-9}-4 / 78$ & 10.3 & 1.5 \\
\hline $4953 \mathrm{C}$ & $10^{-8}-4 / 78$ & 10.3 & 1.3 \\
\hline
\end{tabular}


These samples are contained in flame-sealed glass ampoules.

\begin{tabular}{|c|c|c|c|}
\hline SRM & Nominal Radium Content $(\mu \mathrm{g})$ (Month/Year) & $\begin{array}{l}\text { Approxi- } \\
\text { mate Mass } \\
\text { of Solution } \\
(\mathrm{g})\end{array}$ & $\begin{array}{c}\text { Overall } \\
\text { Uncertainty } \\
(\%)\end{array}$ \\
\hline 4956 & $0.2-9 / 67$ & 5.1 & 4.4 \\
\hline 4957 & $0.5-9 / 67$ & 5.1 & 1.8 \\
\hline 4958 & $1.0-9 / 67$ & 5.1 & 1.8 \\
\hline 4959 & 2.0 & 5.1 & 1.3 \\
\hline 4960 & $5.0-9 / 67$ & 5.1 & 1.3 \\
\hline 4961 & $10.0-9 / 67$ (1) & 5.1 & 1.1 \\
\hline 4962 & $20.0-9 / 67$ & 5.1 & 1.1 \\
\hline 4963 & $50.0-9 / 67$ & 5.1 & 1.1 \\
\hline 4964B & $102.0-6 / 65$ & 5.2 & 0.5 \\
\hline
\end{tabular}

Environmental Standards

\begin{tabular}{|c|c|c|c|}
\hline SRM & Form & Radionuclides & $\begin{array}{l}\text { Ap- } \\
\text { prox. } \\
\text { Mass } \\
\text { (g) }\end{array}$ \\
\hline RM45B & 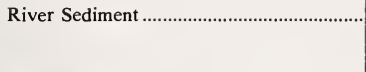 & $\begin{array}{l}{ }^{40} \mathrm{~K},{ }^{54} \mathrm{Mn},{ }^{60} \mathrm{Co},{ }^{35} \mathrm{Zn},{ }^{90} \mathrm{Sr},{ }^{90} \mathrm{Y},{ }^{137} \mathrm{Cs},{ }^{152} \mathrm{Eu},{ }^{154} \mathrm{Eu},{ }^{228} \mathrm{Ac},{ }^{239} \mathrm{Pu} \text {, } \\
{ }^{240} \mathrm{Pu},{ }^{55} \mathrm{Fe},{ }^{125} \mathrm{Sb},{ }^{155} \mathrm{Eu},{ }^{208} \mathrm{Tl},{ }^{212} \mathrm{~Pb},{ }^{212} \mathrm{Bi},{ }^{214} \mathrm{~Pb},{ }^{214} \mathrm{Bi},{ }^{224} \mathrm{Cm},{ }^{226} \mathrm{Ra} \text {, } \\
{ }^{228} \mathrm{Th},{ }^{224} \mathrm{Th},{ }^{232} \mathrm{Th},{ }^{231} \mathrm{~Pa},{ }^{234} \mathrm{U},{ }^{235} \mathrm{U},{ }^{238} \mathrm{U},{ }^{238} \mathrm{Pu},{ }^{241} \mathrm{Am} .\end{array}$ & 100 \\
\hline
\end{tabular}

\section{Metallurgical Standards}

SRM 493 is intended for calibration X-ray diffraction equipment to determine the relative amounts of iron carbide in steel. SRM's $485 \mathrm{a}$ and 488 are intended for the calibration of X-ray diffraction equipment used in determining the amount of retained austenite in ferrous materials.

\begin{tabular}{|c|c|c|}
\hline SRM & Type & Form \\
\hline $\begin{array}{l}485 a \\
486 \\
493\end{array}$ & $\begin{array}{l}\text { Austenite in Ferrite } \sim 5 \% \\
\text { Austenite in Ferrite } \sim 15 \% \\
\text { Spheroidized Iron Carbide }\left(\mathrm{Fe}_{3} \mathrm{C}\right) \text { in Ferrite }\end{array}$ & $\begin{array}{l}\text { IN PREP. } \\
\text { Disk: } 20.6 \mathrm{~mm} \text { dia } \times 2.5 \mathrm{~mm} \text { thick. } \\
\text { Wafer: } 29 \times 29 \times 2.4 \mathrm{~mm} \text {. }\end{array}$ \\
\hline
\end{tabular}

\section{Mössbaver Standards}

These SRM's are issued for the calibration of the isomer shift of iron compounds and alloys and to provide a uniform basis for presentation of mössbauer isomer shift data.

\begin{tabular}{|c|c|c|}
\hline SRM & Type & Form \\
\hline 1541 & (1) & Foil: $2.5 \mathrm{~cm} \times 2.5 \mathrm{~cm} \times 23 \mu \mathrm{m}$. \\
\hline
\end{tabular}




\section{X-ray Fluorescent Emission Target}

This SRM is intended for use in determining the detector window absorption in semiconductor $\mathrm{x}$-ray spectrometers according to ANSI-IEEE Standard STO 759. When excited by a ${ }^{55} \mathrm{Fe}$ source this glass target will emit fluorescent $\mathrm{x}$-rays in the range 1.0 to $5.2 \mathrm{keV}$.

\begin{tabular}{|c|c|c|}
\hline SRM & Type & Unit size \\
\hline 477 & Glass Disk & $2 \times 25 \mathrm{~mm} \mathrm{D}$ \\
\hline
\end{tabular}

\section{X-ray Diffraction Standards}

SRM's 640 and 674 are powdered materials to be used as internal standards for powder diffraction measurements. The lattice parameter of SRM 640 has been accurately determined at $25.0^{\circ} \mathrm{C}$ using a high angle goniometer and the NBS tungsten internal standard. The use of SRM 640 will allow the results to be coupled to Powder Diffraction File (when converted to the same wavelength) base on the NBS internal standards of 1966. The weighted average of the lattice parameter, a, uncorrected for refraction is 5.43088 $\AA$ and the standard error is estimated to be $3.5 \times 10-5 \AA$, SRM 674 is a set of five oxides for use in the quantitative analysis (intensity measurement) of materials. Both peak height and integrated intensity of diffraction lines will be certified.

\begin{tabular}{|c|c|c|}
\hline SRM & Type & Unit size \\
\hline $\begin{array}{l}640 \\
674\end{array}$ & $\begin{array}{l}\text { Silicon Powder } \\
\text { Powder } \mathrm{D}^{2}\end{array}$ & $\begin{array}{l}10 \mathrm{~g} . \\
\text { IN PREP. }\end{array}$ \\
\hline
\end{tabular}

\section{Gas Transmission Standard}

SRM 1470 is for use in the measurement of the oxygen gas transmission rate using a volumetric method (ASTM D1434), manometric method (ASTM D1434 or ISO 2556), or coulometric method of measurement. The oxygen gas transmission rate of SRM 1470 is $0.325 \mathrm{pmol} / \mathrm{m}^{2} / \mathrm{sec} / \mathrm{Pa}$ at $296.16 \mathrm{~K}$ and 0.1013 $\mathrm{MPa}$.

\begin{tabular}{|c|c|c|}
\hline SRM & Type & Unit size \\
\hline 1470 & Polyester Plastic film for oxygen gas transmission & 15 sheets; $23 \mathrm{~cm}$ square. \\
\hline
\end{tabular}




\section{Permittivity Standards}

The three solution SRM's (1511, 1512, and 1513) are for calibrating cells and test capacitors used to determine the relative permittivity (dielectric constant) of liquids. The nominal dielectric constants $(\epsilon)$ for SRM's 1511, 1512, and 1513 are: 2.0, 10.4, and 35.7, respectively. The three polymer SRM's (1516, 1517, and 1519) are for calibrating systems used to measure permittivity and related dielectric quantities. These SRM's are disks of a fluorinated ethylene-propylene copolymer and are individually calibrated.

\begin{tabular}{|c|c|c|}
\hline SRM & Type & Unit size \\
\hline 1511 & Cyclohexane......... & $400 \mathrm{~mL}$. \\
\hline 1512 & 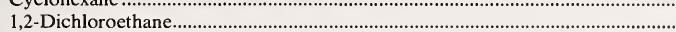 & $400 \mathrm{~mL}$. \\
\hline 1513 & 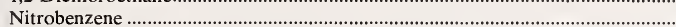 & $400 \mathrm{~mL}$. \\
\hline 1516 & 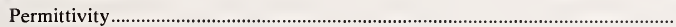 & $38 \mathrm{~mm}$ diameter $2.5 \mathrm{~mm}$ thick. \\
\hline 1517 & Permittivity & $38 \mathrm{~mm}$ diameter $5 \mathrm{~mm}$ thick. \\
\hline 1519 & Permittivity & $51 \mathrm{~mm}$ diameter $5 \mathrm{~mm}$ thick. \\
\hline
\end{tabular}

\section{Reference Fuel Standards}

SRM's 1815 and 1816 are intended for use in maintaining the integrity of motor and aviation fuels as specified in the ASTM Manual for Rating Motor, Diesel and Aviation Fuels, Third Edition.

\begin{tabular}{|c|c|c|}
\hline SRM & Type & Unit \\
\hline $1815 \mathrm{a}$ & n-Heptane & IN PREP. \\
\hline $1816 \mathrm{a}$ & Isooctane $(2,2,4-$ Trimethylpentane) & IN PREP. \\
\hline
\end{tabular}

\section{Resistivity Standards}

SRM's 1460 through 1469 are certified for electrical resistivities over wide temperature ranges for use in calibrating knife edge and similar electric resistivity apparatus. At room temperature these SRM's have the following resistivities: Iron, $10 \cdot 10^{-8} \Omega \cdot \mathrm{m}$, Steel, $81 \cdot 10^{-8} \Omega \cdot \mathrm{m}$, and Tungsten, $6 \cdot 10^{-8} \Omega \cdot \mathrm{m}$.

SRM's 1521 and 1522 Boron-Doped Silicon, are both sets of two single-crystal wafers, with nominal resistivities of 0.1 and $10 \Omega \cdot \mathrm{cm}$, respectively. With the exception of thickness, these two SRM's are physically identical. They are intended for use in calibrating instruments used to measure the resistivity of silicon wafers by four-probe method (ASTM Method F-84).

\begin{tabular}{|c|c|c|}
\hline SRM & Type & Unit of issue \\
\hline 1463 & Electrolytic Iron & Rod $0.64 \mathrm{~cm} \mathrm{dia.} \times 5 \mathrm{~cm}$ long. \\
\hline 1464 & Electrolytic Iron & Rod $3.17 \mathrm{~cm} \mathrm{dia.} \times 5 \mathrm{~cm}$ long. \\
\hline 1460 & Austenitic Stainless Steel & Rod $0.64 \mathrm{~cm}$ dia. $\times 5 \mathrm{~cm}$ long. \\
\hline 1461 & Austenitic Stainless Steel & Rod $1.27 \mathrm{~cm} \mathrm{dia.} \times 5 \mathrm{~cm}$ long. \\
\hline 1462 & 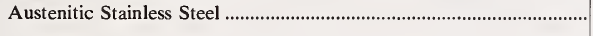 & Rod $3.4 \mathrm{~cm}$ dia. $\times 5 \mathrm{~cm}$ long. \\
\hline 1465 & Tungsten, sintered & $0.32 \mathrm{~cm} \mathrm{dia.} \times 5 \mathrm{~cm}$ long. \\
\hline 1466 & 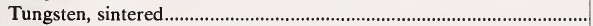 & $0.64 \mathrm{~cm}$ dia. $\times 5 \mathrm{~cm}$ long. \\
\hline 1467 & Tungsten, ARC-CAST & $0.83 \mathrm{~cm}$ dia. $\times 5 \mathrm{~cm}$ long. \\
\hline 1468 & Tungsten, ARC-CAST & $1.02 \mathrm{~cm} \mathrm{dia.} \times 5 \mathrm{~cm}$ long. \\
\hline 1469 & Tungsten, ARC-CAST & $1.27 \mathrm{~cm}$ dia. $\times 5 \mathrm{~cm}$ long. \\
\hline 1521 & Boron-Doped Silicon & 2 Wafers $4.2 \mathrm{~cm}$ dia. $\times<0.74 \mathrm{~mm}$ thick. \\
\hline 1522 & Silicon Power Device & 3 Wafers $5.1 \mathrm{~cm}$ dia. $\times<0.64 \mathrm{~mm}$ thick. \\
\hline
\end{tabular}




\section{ENGINEERING TYPE STANDARDS}

These SRM's are intended to relate measurements used for production or quality control data to a central point of reference. The values certified for these materials are in some cases empirical and do not necessarily relate to the National Measurement System.

\section{Standard Rubbers and Rubber-Compounding Materials}

These SRM's have been prepared to provide the rubber industry with standard materials for rubber compounding. They are useful for the testing of rubber and rubber-compounding materials in connection with quality control of raw materials and for the standardization of rubber testing.

Each material has been statistically evaluated for uniformity by mixing rubber compounds and vulcanizing them in accordance with ASTM Designation D-15 and determining the stress-strain properties of the resulting vulcanizates. Certificates are issued for the rubbers because the properties of different lots are not the same. Replacement lots of rubber-compounding SRM's impart essentially the same characteristics to rubber vulcanizates so that Certificates are not issued for these SRM'S.

\section{Standard Rubbers}

\begin{tabular}{|c|c|c|c|}
\hline SRM & Type & Wt/Unit (grams) & Pounds \\
\hline $385 \mathrm{c}$ & Natural (IN PREP) & 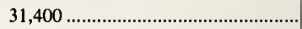 & 69 \\
\hline $386 \mathrm{~h}$ & Styrene-butadiene 1500 & 34,000 & 75 \\
\hline $388 \mathrm{k}$ & Butyl & 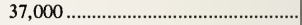 & 81.5 \\
\hline
\end{tabular}

\section{Rubber Compounding Materials}

\begin{tabular}{|c|c|c|c|}
\hline SRM & Type & Wt/Unit (grams) & Pounds \\
\hline $370 \mathbf{e}$ & 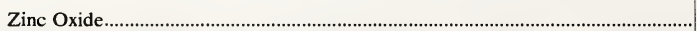 & 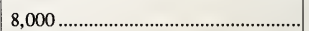 & 17.6 \\
\hline $371 \mathrm{~g}$ & Sulfur & 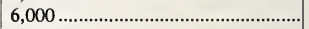 & 13.2 \\
\hline $372 \mathrm{~h}$ & 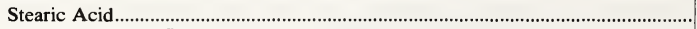 & 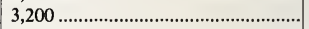 & 7.1 \\
\hline $373 \mathrm{f}$ & Benzothiazyl disulfide & 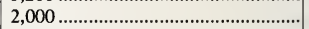 & 4.4 \\
\hline $374 \mathrm{c}$ & Tetramethylthiuram disulfide & 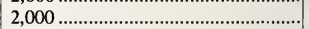 & 4.4 \\
\hline $375 \mathrm{~g}$ & Channel Black & 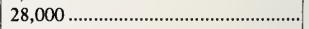 & 61.6 \\
\hline $378 \mathrm{~b}$ & 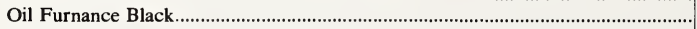 & 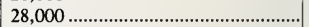 & 61.6 \\
\hline 379 & Conducting Black & 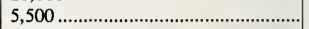 & 12.1 \\
\hline $382 \mathrm{a}$ & Gas Furnace Black & 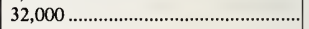 & 70.6 \\
\hline $384 d$ & N-tertiary-Butyl-2-benzothiazolesulfenamide & 3,200 & 7.1 \\
\hline 391 & Acrylonitrile-Butadiene & 25,000 & 5.5 \\
\hline
\end{tabular}

\section{Reference Magnetic Tapes}

These SRM's are intended for use in evaluating the performance of magnetic computer tapes and maintaining control over their production. Each SRM is individually calibrated and certified.

\begin{tabular}{|c|c|c|}
\hline SRM & Type & Unit \\
\hline 1600 & Secondary standard magnetic tape-computer amplitude reference .... & Cassette. \\
\hline 3200 & Secondary standard magnetic tape-computer amplitude reference & Reel/600 ft. \\
\hline 3210 & Secondary standard flexible disk cartridge-computer amplitude reference ............................. & Flexible disk. \\
\hline 3216 & Secondary standard magnetic tape-computer amplitude reference & Cartridge. \\
\hline 6250 & Secondary standard high density magnetic tape-computer amplitude reference .................... & Reel $/ 2400 \mathrm{ft}$. \\
\hline
\end{tabular}




\section{Centerline Drawings for Optical Character Recognition-B Characters}

This SRM is an exact copy of the centerline drawings that uniquely define each printed character shape and size used in constant strokewidth Style B Optical Character Recognition (OCR-B) applications in accordance with one or more of the following standards: American National Standard X3.49-1975, Character Set for Optical Character Recognition (OCR-B); Federal Information Processing Standards Publication 32-1974, Optical Character Recognition Character Sets; European Computer Manufacturers Association Standard ECMA-II for the Alphanumeric Character Set OCR-B for Optical Recognition, 3rd Edition, 1975; and Draft International Standard ISO/DIS 1073/II, Alphanumeric Character Sets for Optical Recognition.

This standard contains information on the nominal size, strokewidth, tolerance, and relative position of characters.

\begin{tabular}{c|c|c}
\hline SRM & Size & OCR-B Characters \\
\hline 1901 & I & 118 \\
\hline
\end{tabular}

Sizing Standards

Glass Spheres for Particle Size

\begin{tabular}{|c|c|c|c|c|}
\hline SRM & Type & Size $(\mu \mathrm{m})$ & Sieve Nos. & $\begin{array}{l}\text { Wt/Unit } \\
\text { (grams) }\end{array}$ \\
\hline 1003 & Calibrated Glass Spheres.... & $5-30$ & & $40-45$ \\
\hline 1004 & 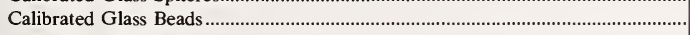 & $34-120$ & $400-140$ & 63 \\
\hline $1017 \mathrm{a}$ & Calibrated Glass Beads & $100-310$ & $140-50$ & 84 \\
\hline $1018 \mathbf{a}$ & Calibrated Glass Beads & $225-780$ & $60-25$ & 74 \\
\hline $1019 \mathrm{a}$ & 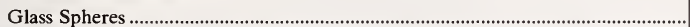 & IN PREP & 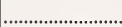 & \\
\hline
\end{tabular}

Turbidimetric and Fineness Standard (Cement)

This SRM is available to calibrate the Blaine fineness meter according to the latest issue of Federal Test Method Standard 158, Method 2101 or ASTM Designation C204; to calibrate the Wagner turbidimeter according to ASTM Designation C115; and to determine sieve residue according to ASTM Designation C430. Each set consists of twenty sealed vials, each containing approximately 10 grams of cement. This SRM is supplied only in sets of twenty vials or multiples thereof.

\begin{tabular}{|c|c|c|c|}
\hline SRM & Type & Certification & Unit \\
\hline $114 \mathrm{~m}$ & Portland Cement & 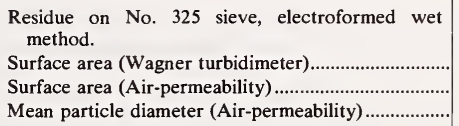 & Set of 20 vials. \\
\hline
\end{tabular}

\section{Color Standards}

These SRM's are available to illustrate a characteristic color for each of the ISCC-NBS color-name blocks in NBS Special Publication 440, COLOR: Universal Language and Dictionary of Names. SRM 2106 consists of 251 color chips on 18 constant-hue centroid color charts, and constitutes a supplement to SP 440. SRM 2107 combines SRM 2106 with SP 440 to form a complete color kit. The centroid colors 
represent a systematic sampling of the whole color solid, each color of which has been carefully measured. Each centroid color has its own specification and can be used as a color standard. (NOTE: SP 440, may be purchased separately from the Superintendent of Documents, U.S. Government Printing Office, Washington, D.C. 20402.)

\begin{tabular}{|c|c|c|}
\hline SRM & Type & Unit of Issue \\
\hline 2106 & 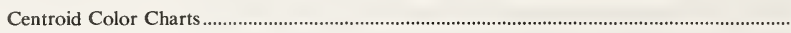 & Set: 18 Charts. \\
\hline 2107 & Color Kit..1. & Set: SRM 2106 and SP 440. \\
\hline
\end{tabular}

\section{Light-Sensitive Papers and Plastic Chips}

Light-Sensitive Papers

Standard light-sensitive paper and booklets of standard faded strips of this paper are available for use in standardizing the dosage of radiant energy when testing textiles for color fastness by exposure in commercial carbon-arc fading lamps. The paper is distributed in units of 100 pieces $2 \frac{5}{8}$ by $3 \frac{1}{4}$ in. The booklet contains six strips of the paper $1 \frac{1 / 4}{4}$ in wide that have been faded by exposure in the NBS master lamp. A copy of NBS Spec. Publ. 260-41, which describes the preparation and use of the materials, is furnished with each booklet.

\begin{tabular}{|c|c|c|}
\hline SRM & Type & Unit of Issue \\
\hline $\begin{array}{l}700 \mathrm{~d} \\
701 \mathrm{~d}\end{array}$ & Light-sensitive paper & $\begin{array}{l}\text { Pkg. of } 100 \text { pieces }-2 \frac{5}{8} \text { in } \times 31 / 4 \text { in. } \\
\text { Booklet }-6 \text { strips } 1 \frac{11 / 4}{\text { in wide. }}\end{array}$ \\
\hline
\end{tabular}

\section{Light-Sensitive Plastic Chips}

Standard light-sensitive plastic chips are available for use in calibration and standardization of artificial weathering and fading apparatus. These chips are distributed in two thicknesses (0.060 and 0.124 in) in units of five plates 2 in by $4 \frac{1}{8}$ in, and have been standardized by the measurement of the change of transmittance as a function of exposure (in standard fading hours) to the NBS master lamps.

\begin{tabular}{|c|c|c|}
\hline SRM & Type & Unit of Issue \\
\hline $\begin{array}{l}702 \\
703\end{array}$ & $\begin{array}{l}\text { Light-sensitive plastic chips } \\
\text { Light-sensitive plastic chips }\end{array}$ & $\begin{array}{l}\text { Package of } 5 \text { chips } 0.124 \text { in thick. } \\
\text { Package of } 5 \text { chips } 0.060 \text { in thick. }\end{array}$ \\
\hline
\end{tabular}

\section{$X-R a y$ and Photographic Standards}

SRM 1001 is a calibrated X-ray film step tablet of 17 steps that cover the optical density range from 0 to 4. SRM 1008 is a calibrated photographic step tablet of 21 steps that cover the optical density range from 0 to 4 .

Both step tablets are individually calibrated and certified for diffuse transmission density in conformance with conditions specified for American National Standard Diffuse Visual Transmission Density, $D_{t}$ (90; $3000 \mathrm{~K}: \leqslant 10^{\circ}$; V), in "ANSI PH2.19-1976, American National Standard for Diffuse and Doubly Diffuse Transmission Measurements (Transmission Density)."

SRM 1010a, Microcopy Resolution Test Charts, is used to test the resolving power of cameras or of whole microcopying systems. SRM 1010a consists of five charts printed photographically on paper, and 
have 26 high-contrast five-line patterns ranging in spatial frequency from one cycle per millimeter to 18 cycles per millimeter. Instructions for the use of the charts are supplied with each order.

\begin{tabular}{|c|c|c|}
\hline SRM & Type & Unit \\
\hline $\begin{array}{l}1001 \\
1008 \\
1010 \mathrm{a}\end{array}$ & $\begin{array}{l}\text { X-ray Flim Step Tablet (0-4) } \\
\text { Photographic Step Tablet (0-4) } \\
\text { Microcopy Resolution Test Chart }\end{array}$ & $\begin{array}{l}1 \text { tablet, } 17 \text { steps. } \\
1 \text { tablet, } 21 \text { steps. } \\
\text { Set of } 5 \text { charts. }\end{array}$ \\
\hline
\end{tabular}

\section{Surface Flammability Standard}

SRM 1002c, Hardboard Sheet, is issued for checking the operation of radiant panel test equipment in accordance with the procedures outlined in ASTM Standard E162-78.

\begin{tabular}{|c|c|c|c|}
\hline SRM & Type & Certification & Unit of Issue \\
\hline $1002 \mathrm{c}$ & Hardboard Sheet ........ & $\begin{array}{l}\text { Flame Spread Index, } \mathrm{I}=153 \\
\text { Heat Evolution Factor, } \mathrm{Q}=36.5\end{array}$ & Set of $4: 6 \times 18 \times 1 / 4$ inch. \\
\hline
\end{tabular}

\section{Smoke Density Chamber Standards}

These SRM's are certified for maximum specific optical density and are issued for performing operational checks of smoke density chambers.

\begin{tabular}{|c|c|c|c|}
\hline SRM & Type & Maximum Specific Optical Density & Unit of Issue \\
\hline $1006 \mathrm{a}$ & 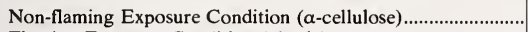 & $\operatorname{Dm}($ corr.$)=161 \ldots \ldots \ldots \ldots \ldots$ & 3 sheets. \\
\hline $1007 \mathrm{a}$ & 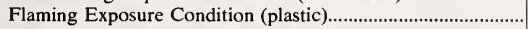 & $\operatorname{Dm}($ corr. $)=17850(t)-132 \ldots \ldots$ & 3 sheets. \\
\hline
\end{tabular}

\section{Tape Adhesion Testing Standard}

This material is intended as a uniform source of linerboard for use under ASTM Designation D2860, Procedure A: Adhesion of Pressure Sensitive Tape to Fiberboard at 90 Degree Angle and Constant Stress.

\begin{tabular}{l|c|c}
\hline SRM & Type & Unit \\
\hline 1810 & $\begin{array}{l}\text { Linerboard for Tape Adhe- } \\
\text { sion Testing. }\end{array}$ & Package of 50 sheets. \\
\hline
\end{tabular}


Research Materials (RM's) are in addition to and distinct from the Standard Reference Materials (SRM's) issued by NBS. The distinctions between Research Materials and Standard Reference Materials are in the information supplied with them and purpose for which they are used. Unlike SRM's, the RM's are not issued with Certificates of Analysis; rather they are accompanied by a "Report of Investigation," the sole authority of which is the author of the report. A Research Material is intended primarily to further scientific or technical research on that particular material. One of the principal considerations in issuing an RM is to provide homogeneous material so that an investigator in one laboratory can be assured that the material he has is the same as that being investigated in a different laboratory.

RM-1C Ultra-purity aluminum single crystal cubes (1 $\mathrm{cm}$ on a side) are intended for use in studies of a variety of solid state phenomena for which both extreme purity and knowledge of crystallographic orientation are required; e.g., in studies of electron spin resonance, De Haas-Van Alphen effect, cyclotron resonance, and in a variety of studies relating to the Fermi surface and the transport properties of aluminum.

RM-1R Ultra-purity aluminum polycrystalline rods $(4.2 \mathrm{~mm}$ in diameter and $25.4 \mathrm{~mm}$ long) are intended for use in research on the mechanical and physical properties of extremely pure aluminum: e.g., in the determination of resistivity as a function of strain at cryogenic temperatures to facilitate the design of cryogenic magnets or superconductor stabilizing elements.

RM 5 Copper Heat Capacity Test Specimen. This Research Material is intended for the comparison of heat capacity results from different laboratories and as a test specimen for heat capacity measurement below $25 \mathrm{~K}$, but it may also be useful at higher temperatures. It is available as a rod of high purity polycrystalline copper $19 \mathrm{~mm}(0.75 \mathrm{in})$ in diameter and $120 \mathrm{~mm}(4.75 \mathrm{in})$ in length.

RM 31 Glass Fibers for Microanalysis (RM 31). This homogeneous vitreous solid contains known, low-concentration additions of several elements which were developed for electron probe microanalysis (EMPA) and secondary ion mass spectrometry (SIMS). This RM contains ten compositions of various oxides: in fiber form.

RM 40 Polystyrene solution in a mixture of tri-cresyl-phosphate and Aroclor. This Research Material can be used to check instruments used in polymer technology and science for the determination of rheological properties of polymer melts or solutions. It was designed so that the limiting viscosity is obtained at $25^{\circ} \mathrm{C}$ at shear rates as high as 1 reciprocal second. Values are given for rate of shear, viscosity, and first and second normal stress difference at $25^{\circ} \mathrm{C}$. Unit of issue: $250 \mathrm{~mL}$.

RM 45b Homogeneous River Sediment. This Research Material should be particularly useful for testing radiochemical procedures for the assay of radioactivity in sediments and soils. Unit of issue: 100 grams.

RM 50 Albacore Tuna. This Research Material is intended to be used in the measurement of elements present at trace concentration. It has been issued as a lyophilized (freeze-dried) marine biological tissue sample in an attempt to satisfy many of the analytical requirements for a base-line marine reference material. The Report of Investigation provides informational data (not certified) on mercury, selenium, zinc, arsenic, lead, and a number of other elements of interest to marine scientists. Unit of issue: two $35-\mathrm{g}$ cans.

\section{Phosphors}

These materials are issued without Certification. NBS Technical Note 417, Spectral Emission Properties of NBS Standard Phosphor Samples Under Photo-Excitation, is issued with these materials, and is 
equivalent to the "Report of Investigation" issued with Research Materials. They are issued so that those interested in developing methods of measurement for phosphor materials can work on a common source of materials. NBS Technical Note 417 may be purchased from Superintendent of Documents, U.S. Government Printing Office, Washington, D.C. 20402, for 25 cents, by SD Catalog No. C13.46:47.

\begin{tabular}{|c|c|c|}
\hline SRM & Type & $\begin{array}{l}\text { Wt/Unit } \\
\text { (grams) }\end{array}$ \\
\hline 1020 & Zine sulfide phosphor & 14 \\
\hline 1021 & 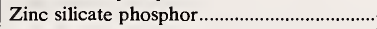 & 28 \\
\hline 1022 & Zinc sulfide phosphor & 14 \\
\hline 1023 & $\begin{array}{l}\text { Zinc-cadmium sulfide phosphor (AG acti- } \\
\text { vator). }\end{array}$ & 14 \\
\hline 1024 & $\begin{array}{l}\text { Zinc-cadmium sulfide phosphor (Cu acti- } \\
\text { vator). }\end{array}$ & 14 \\
\hline 1025 & 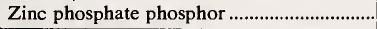 & 28 \\
\hline
\end{tabular}

\begin{tabular}{|c|c|c|}
\hline SRM & Type & $\begin{array}{l}\text { Wt/Unit } \\
\text { (grams) }\end{array}$ \\
\hline 1026 & 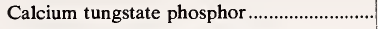 & 28 \\
\hline 1027 & Magnesium tungstate phosphor............... & 28 \\
\hline 1028 & 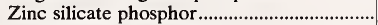 & 28 \\
\hline 1029 & Calcium silicate phosphor................................. & 14 \\
\hline 1030 & Magnesium arsenate phosphor .......................... & 28 \\
\hline 1031 & Calcium halophosphate phosphor .................... & 28 \\
\hline 1032 & Barium silicate phosphor ................................... & 28 \\
\hline 1033 & Calcium phosphate phosphor ........................... & 28 \\
\hline
\end{tabular}




\section{SPECIAL REFERENCE MATERIALS}

Special Reference Materials (GM's) are distributed by NBS to meet industry needs. These materials have been standardized either by some Government agency other than NBS, or by some standards-making body such as the American Society for Testing and Materials (ASTM), the American National Standards Institute (ANSI), and the Organization for International Standardization (ISO). For this class of materials, NBS acts only as a distribution point and does not participate in the standardization of these materials.

GM-1 Hydrogen in Steel Standards were produced and certified by the Welding Institute in Cambridge, England, and are distributed in the United States by NBS. GM-1 is a set of 15 cylinders, 5 each of $\mathrm{H} 1, \mathrm{H} 2$, and $\mathrm{H} 3$, containing nominally $0.05,0.10$, and $0.20 \mathrm{~mL}$ hydrogen, respectively. The cylinders are $6.35 \mathrm{~mm}$ in diameter and about $30 \mathrm{~mm}$ long, weighing approximately 6 grams.

GM-2 Hydrogen in Steel Standards were produced and certified by the Welding Institute in Cambridge, England, and are distributed in the United States by NBS. GM-2 is a set of 15 cylinders, 5 each of $\mathrm{H} 4, \mathrm{H} 5$, and $\mathrm{H} 6$, containing nominally $0.20,0.60$, and $1.10 \mathrm{~mL}$ hydrogen, respectively. The cylinders are $12.7 \mathrm{~mm}$ in diameter and about $30 \mathrm{~mm}$ long, weighing approximately 22 grams.

GM-5 Nickel and Vanadium in Residual Oil was produced and analyzed under the sponsorship of the Western Oil and Gas Association and the American Petroleum Institute, and is distributed by NBS. The assigned values for nickel and vanadium are 93 and $79 \mathrm{ppm}$, respectively. GM-5 is issued in $475 \mathrm{~mL}$ units.

GM-6 This GM, DOT 6M Shipping Container, is manufactured in accordance with the applicable DOT specifications, the Nuclear Regulatory Commission Quality Assurance Program Requirements, and the National Bureau of Standards specifications. This container is to be used in the transport of plutonium or all type $\mathbf{B}$ quantities of fissile or nonfissile radioactive materials in special or non-special form.

When plutonium materials are ordered from NBS, certified containers may be supplied by customers or these GM's may be purchased from NBS. These containers may be reused for additional orders to NBS of subsequent materials.

GM-21 Cellular Plastics. These GM's are to be used in comparison of fire research data. Each of these GM-57 GM's is shipped FOB: Gaithersburg, MD.

\begin{tabular}{|c|c|c|c|c|c|}
\hline GM & Type & $\begin{array}{l}\text { Density } \\
\left(\mathrm{lb} / \mathrm{ft}^{3}\right)\end{array}$ & $\begin{array}{l}\text { Thick- } \\
\text { ness } \\
\text { (inches) }\end{array}$ & $\begin{array}{c}\text { Size } \\
\text { Width } \\
\text { (ft) }\end{array}$ & $\begin{array}{l}\text { Length } \\
\text { (ft) }\end{array}$ \\
\hline 21 & Flexible Polyurethane ................ & 2 & 6 & 2 & 2 \\
\hline 23 & Flexible Polyurethane, FR & 2 & 6 & 2 & 2 \\
\hline 25 & Flexible Polyurethane, HR & 4 & 6 & 2 & 2 \\
\hline 27 & Flexible Polyurethane, HR/FR & 4 & 6 & 2 & 2 \\
\hline 29 & Rigid Polyurethane & 2 & 4 & 2 & 2 \\
\hline 31 & Rigid Polyurethane, FR $>25$ FS & 2 & 4 & 2 & 2 \\
\hline 35 & Rigid Polyurethane, HR & 4 & 6 & 2 & 2 \\
\hline 37 & Rigid Polyurethane, HR/FR & 20 & 6 & 2 & 2 \\
\hline 39 & Rigid Polyurethane sprayed on Asbestos Cement Board, $>25$ FS........................... & 2 & 1.5 & 4 & 4 \\
\hline 40 & Rigid Polyurethane sprayed on Asbestos Cement Board, $<25$ FS & 2 & 1.5 & 4 & 4 \\
\hline 41 & Rigid Trimer & 2 & 4 & 2 & 2 \\
\hline 43 & Rigid Trimer, $\sim 25$ FS & 2 & 4 & 2 & 2 \\
\hline 47 & Polystyrene Expanded & 1 & 4 & 2 & 2 \\
\hline 49 & Polystyrene Expanded, FR & 1 & 4 & 2 & 2 \\
\hline 51 & Polystyrene Extruded & 1 & 3 & 2 & 2 \\
\hline 53 & Polystyrene Extruded, FR & 1 & 3 & 2 & 2 \\
\hline 57 & 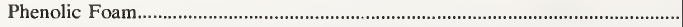 & 2 & 4 & 2 & 2 \\
\hline
\end{tabular}


GM's $754,757,758,759$, and 760 have been issued by NBS in cooperation with the International Confederation of Thermal Analysis as standards for calibrating differential thermal anaylsis and related thermoanalytical equipment under operating conditions.

\begin{tabular}{|c|c|c|c|}
\hline GM & Material & Peak Temp. $\left({ }^{\circ} \mathrm{C}\right)$ & Unit \\
\hline 754 & Polystyrene ............... & 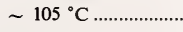 & $10 \mathrm{~g}$. \\
\hline \multirow[t]{5}{*}{757} & 1,2-Dichloroethane .............. & $\sim-32{ }^{\circ} \mathrm{C} \ldots \ldots \ldots \ldots . . . . .$. & $4 \mathrm{~mL}$. \\
\hline & Cyclohexane: & & \\
\hline & (transition) & $\sim-83^{\circ} \mathrm{C} \ldots \ldots \ldots \ldots \ldots . . . . . . . .$. & $4 \mathrm{~mL}$. \\
\hline & (melting) & 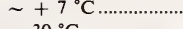 & \\
\hline & $\begin{array}{l}\text { Phenyl Ether } \\
\text { o-Terphenyl }\end{array}$ & 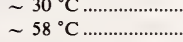 & $\begin{array}{l}4 \mathrm{~mL} \text {. } \\
5 \mathrm{~g} .\end{array}$ \\
\hline \multirow[t]{5}{*}{758} & 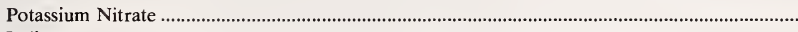 & $\sim 128{ }^{\circ} \mathrm{C} \ldots \ldots .$. & $10 \mathrm{~g}$. \\
\hline & Indium & $\sim 157^{\circ} \mathrm{C}$ & $3 \mathrm{~g}$. \\
\hline & Tin & 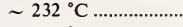 & $3 \mathrm{~g}$. \\
\hline & Potassium Perchlorate & $\sim 300^{\circ} \mathrm{C} \ldots \ldots \ldots \ldots \ldots \ldots$ & $10 \mathrm{~g}$. \\
\hline & Silver Sulfate & $\sim 430^{\circ} \mathrm{C} \ldots \ldots \ldots \ldots \ldots . . . . . . . . .$. & $3 \mathrm{~g}$. \\
\hline \multirow[t]{5}{*}{759} & Potassium Perchlorate & $\sim 300^{\circ} \mathrm{C} \ldots$ & $10 \mathrm{~g}$. \\
\hline & 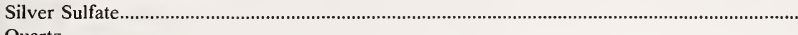 & $\sim 430^{\circ} \mathrm{C} \ldots$. & $3 \mathrm{~g}$. \\
\hline & Quartz & $\sim 573^{\circ} \mathrm{C} \ldots \ldots \ldots \ldots \ldots \ldots$ & $3 \mathrm{~g}$. \\
\hline & Potassium Sulfate & $\sim 583^{\circ} \mathrm{C} . . . \ldots \ldots \ldots \ldots . . . . . . . .$. & $10 \mathrm{~g}$. \\
\hline & 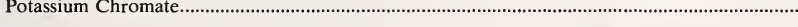 & $\sim 665^{\circ} \mathrm{C}$ & $10 \mathrm{~g}$. \\
\hline \multirow[t]{5}{*}{760} & Quartz & $\sim 573^{\circ} \mathrm{C} \ldots \ldots \ldots \ldots \ldots . . . . . . .$. & $3 \mathrm{~g}$. \\
\hline & Potassium Sulfate & $\sim 583^{\circ} \mathrm{C} \ldots \ldots \ldots \ldots \ldots \ldots . . . . . . . .$. & $10 \mathrm{~g}$. \\
\hline & Potassium Chromate & $\sim 665^{\circ} \mathrm{C}$ & $10 \mathrm{~g}$. \\
\hline & Barium Carbonate & $\sim 810^{\circ} \mathrm{C} \ldots \ldots \ldots \ldots \ldots . . . . . . .$. & $10 \mathrm{~g}$. \\
\hline & Strontium Carbonate & $\sim 925^{\circ} \mathrm{C} \ldots \ldots \ldots \ldots \ldots . . . . . . .$. & $10 \mathrm{~g}$. \\
\hline
\end{tabular}


When the current supplies are exhausted, the SRM's listed below will be discontinued, because of limited user demand or technological obsolescence.

\begin{tabular}{l|l}
\hline SRM & \multicolumn{1}{|c}{ Type } \\
\hline $404 \mathrm{a}$ & Steel, basic electric \\
$405 \mathrm{a}$ & Steel, medium manganese \\
$407 \mathrm{a}$ & Steel, chromium-vanadium \\
$408 \mathrm{a}$ & Steel, chromium-nickel \\
$409 \mathrm{~b}$ & Steel, nickel \\
413 & Steel, A.O.H. 0.4C \\
414 & Steel, Cr-Mo (SAE 4140) \\
$417 \mathrm{a}$ & Steel, B.O.H. 0.4C \\
$418 \mathrm{a}$ & Steel, Cr-Mo (SAE X4130) \\
$420 \mathrm{a}$ & Iron, ingot \\
427 & Steel, Cr-Mo (boron only) (SAE 4150) \\
436 & Steel, special Cr6-Mo3-W10 \\
437 & Steel, special Cr8-Mo2-W3-Co3 \\
438 & Steel, Mo high speed (AISI-SAE-M30) \\
439 & Steel, Mo high speed (AISI-SAE-M36) \\
440 & Steel, special W high speed Cr2-W13-Co12 \\
441 & Steel, W high speed (AISI-SAE-T1) \\
442 & Steel, stainless, Cr16-Ni10 \\
443 & Steel, stainless, Cr18.5-Ni9.5 \\
444 & Steel, stainless, Cr20.5-Ni10 \\
445 & Steel, stainless, Cr13-Mo0.9 (Modified AISI 410) \\
446 & Steel, stainless, Cr18-Ni9 (Modified AISI 321) \\
447 & Steel, stainless, Cr24-Ni13 (Modified AISI 309) \\
448 & Steel, stainless, Cr9-Mo0.3 (Modified AISI 403) \\
449 & Steel, stainless, Cr5.5-Ni6.5 \\
450 & Steel, stainless, Cr3-Ni25 \\
$803 \mathrm{a}$ & Steel, A.O.H. 0.6C \\
D803a & Steel, A.O.H. 0.6C \\
$804 \mathrm{a}$ & Steel, basic electric \\
$805 \mathrm{a}$ & Steel, medium manganese \\
$807 \mathrm{a}$ & Steel, chromium-vanadium \\
D807a & Steel, chromium-vanadium \\
$808 \mathrm{a}$ & Steel, chromium-nickel \\
& \\
\hline & \\
\hline
\end{tabular}

\begin{tabular}{l|l}
\hline \multicolumn{1}{c|}{ SRM } & \multicolumn{1}{|c}{ Type } \\
\hline $817 \mathrm{a}$ & Steel, B.O.H. 0.4C \\
$820 \mathrm{a}$ & Iron, ingot \\
821 & Steel, Cr-W, 0.9C \\
827 & Steel, Cr-Mo (boron only) (SAE 4150) \\
837 & Steel, special (Cr8-Mo2-W3-Co3) \\
D837 & Steel, special (Cr8-Mo2-W3-Co3) \\
840 & Steel, special W high speed (Cr2-W13-Co12) \\
D840 & Steel, special W high speed (Cr2-W13-Co12) \\
D841 & Steel, W high speed (AISI-SAE-T1) \\
849 & Steel, Cr5.5-Ni6.5 \\
D849 & Steel, Cr5.5-Ni6.5 \\
850 & Steel, Cr3-Ni25 \\
D850 & Steel, Cr3-Ni25 \\
1020 & Zinc sulfide phosphor \\
1021 & Zinc silicate phosphor \\
1022 & Zinc sulfide phosphor \\
1023 & Zinc-cadmium sulfide phosphor (Ag activator) \\
1024 & Zinc-cadmium sulfide phosphor (Cu activator) \\
1025 & Zinc phosphate phosphor \\
1026 & Calcium tungstate phosphor \\
1027 & Magnesium tungstate phosphor \\
1028 & Zinc silicate phosphor \\
1029 & Calcium silicate phosphor \\
1030 & Magnesium arsenate phosphor \\
1031 & Calcium halophosphate phosphor \\
1032 & Barium silicate phosphor \\
1033 & Calcium phosphate phosphor \\
1511 & Cyclohexane, dielectric \\
1512 & 1,2 Dichloroethane, dielectric \\
1513 & Nitrobenzene, dielectric \\
1516 & permittivity std., 38 mm x 2.5 mm \\
1517 & Permittivity std., 38 mm x 5 mm \\
1519 & Permittivity std., 51 mm x 5 mm \\
& \\
\hline &
\end{tabular}


The following is a list of some of the services offered by NBS that may be of interest to SRM users. For general information see the entry on Technical Information and Publications.

\section{Calibration and Related Measurement Services of the National Bureau of Standards}

The measurement services of the National Bureau of Standards include the calibration of standards, test of instruments, and certain interlaboratory testing programs. These services are listed in NBS Special Publication 250, Calibration and Related Measurement Services of the National Bureau of Standards. [Available from the Superintendent of Documents, U.S. Government Printing Office, Washington, D.C. 20402 (1980 edition) for $\$ 4.50]$.

These services are performed at the National Bureau of Standards Washington laboratories (Gaithersburg, Md.) or the National Bureau of Standards laboratories in Boulder, Colorado.

An abbreviated list of the services offered under this program is given below. For information concerning services not listed below or in Special Publication 250 contact:

Office of Measurement Services

National Bureau of Standards

Washington, D.C. 20234

Telephone: (301) 921-2805

\section{Washington Services}

Acoustics

Aerodynamics

Angular

Computer Science (general)

Density

Dosimetry in High-Energy Electron Beams

Electrical Instruments (AC)

Electrical Instruments (DC)

Engineering Tests
(301) 921-3607

(301) 921-3684

(301) 921-2216

(301) 921-3723

(301) 921-3681

(301) 921-2361

(301) 921-3121

(301) 921-2715

(301) 921-3471

(301) 921-2216

Flatness.

Flow Rate Meters

Fluid Quantity

Force Measurements.

Gamma-Ray Sources

Humidity.

Hydraulics

Image Optics

Impedance

Interlaboratory Testing Programs

Length.

Mass

Neutron Sources

Photography.

Photometric Calibrations.
(301) 921-3481

(301) 921-3681

(301) 921-3884

(301) 921-2361

(301) 921-2794

(301) 921-2124

(301) 921-3550

(301) 921-2715

(301) 921-2946

(301) 921-2216

(301) 921-3681

(301) 921-2234

(301) 921-3550

(301) 921-3613 
Precision Apparatus

(301) 921-2715

Proving Rings

(301) 921-3884

Radiation Thermometry

(301) 921-3613

Radioactivity

(301) 921-2665

Radiometric Calibrations

(301) 921-3613

Resistance

(301) 921-2715

Resistance Thermometers

(301) 921-2757

Roundness.

(301) 921-2216

Spectrophotometric Standards

(301) 921-2453

Straightness

(301) 921-2216

Surface Texture

(301) 921-2159

Thermocouples and Thermocouple Materials

(301) 921-2069

Thermometers, Laboratory

(301) 921-2087

Ultraviolet Spectral Radiance Standard

(301) 921-2011

Vibration.

(301) 921-3607

Voltage, High

(301) 921-3121

Voltage

(301) 921-2715

Weights and Measure

(301) 921-2401

$\mathrm{X}$ - and Gamma-Ray Measuring Instruments

(301) 921-2361

\section{Boulder Services}

All measurement services available in Boulder should be directed to:

Measurement Services Clerk

National Bureau of Standards

Boulder, Colorado 80303

Telephone: (303) 499-1000, ext. 3753

Cryogenics

Electromagnetics (Radio, Microwave, and Laser Frequencies)

1. Attenuation

2. Fields (Electromagnetic)

3. Impedance

4. Laser Parameters

5. Noise Temperature (Effective)

6. Phase Shift

7. Power

8. Voltage

Time and Frequency Measurements 
The Standards Information Service (SIS) maintains a reference collection of some 240,000 engineering standards issued by U.S. technical societies, professional organizations, and trade associations; State purchasing offices; U.S. civilian government agencies; and the major foreign national and international standardizing bodies. The collection is open to the public Monday through Friday from 8:30 a.m. to 5 p.m.

SIS publishes general and special indices of standards. Information services consist of responding to inquiries by searching Key-Word-In-Context (KWIC) Indices to determine whether there are any published standards, specifications, test methods, or recommended practices for a given item, product, or material. Inquirers are referred to the appropriate source to obtain copies of standards. SIS neither sells nor distributes standards.

Inquiries or requests for additional information should be directed to:

\author{
Standards Information Service \\ Room B162, Technology Building \\ National Bureau of Standards \\ Washington, D.C. 20234 \\ Telephone: (301) 921-2587
}

\title{
Standard Reference Data
}

The National Standard Reference Data System (NSRDS) is a nationwide program established to make critically evaluated data in the physical sciences available to the technical community. It publishes compilations of critically evaluated data, critical reviews and bibliographies. A complete listing of the publications of the NSRDS is available from the Office of Standard Reference Data (OSRD). The OSRD responds in a limited way to queries within the scope of the program by providing references, referrals, documentation, or data, as available. The program's bimonthly newsletter is available on request. Inquiries or requests for further information should be directed to:

\author{
Information Services \\ Office of Standard Reference Data Reference Center \\ Room A320, Physics Building \\ National Bureau of Standards \\ Washington, D.C. 20234 \\ Telephone: (301) 921-2228
}

\section{Technical Information and Publications}

The Technical Information and Publications Division maintains a general correspondence and inquiry service on the technical activities of the National Bureau of Standards. Inquiries of a general nature and not covered by the services listed above should be directed to:

\author{
Technical Information and Publications Division \\ Room A617, Administration Building \\ National Bureau of Standards \\ Washington, D.C. 20234 \\ Telephone: (301) 921-2318
}




\section{GUIDE FOR REQUESTING DEVELOPMENT OF STANDARD REFERENCE MATERIALS}

The National Bureau of Standards has the function to develop, produce, and distribute Standard Reference Materials (SRM's) that provide a basis for comparison of measurements on materials and that aid in the control of production processes. To perform this function, the Office of Standard Reference Materials evaluates the requirements of science, industry, and government for carefully characterized reference materials, and directs their production and distribution.

NBS currently has over 1000 SRM's available, about 100 new ones in preparation, and requests for the preparation of many others.

In developing and NBS-SRM, the candidate material must meet one or more of the criteria listed below.

1. The SRM must permit users to attain more accurate measurements.

2. The production of the SRM elsewhere is not economically or technically feasible.

3. The SRM would be an industry-wide standard for commerce from a neutral source not otherwise available.

4. NBS production of the SRM would provide continued availability from a common source for a highly characterized material that is important to science, industry, or government.

NBS has recognized and responded to the need to enlarge the scope of the program to include all types of well-characterized materials that can be used to calibrate a measurement system or to produce scientific data that can be readily referred to a common base. However, the demand for new SRM's greatly exceeds the Bureau's capacity to produce and certify these materials. Consequently, requests for new SRM's that would have limited use, or for which the need is not very great, are deferred in favor of requests that clearly show a critical need. To determine which requests are to receive top priority, NBS needs and heavily relies upon the information supplied by industry, either through its own representatives or through interested organizations, such as the American National Standards Institute, American Nuclear Society, American Petroleum Institute, American Society for Testing and Materials, etc.

Accordingly, while the Bureau welcomes all requests for the development of new SRM's, both the Bureau and industry would be helped, if such requests are accompanied by information that will permit an objective assessment of the urgency and importance of proposed new reference materials.

Requests for the development of new Standard Reference Materials should include as much of the information listed below as possible.

1. Short title of the proposed Standard Reference Material.

2. Purpose for which the SRM would be used.

3. Reasons why the SRM is needed.

4. Special characteristics and/or requirements for the material. Include additional requirements and reasons, if more than one SRM is necessary for standardization in this area.

5. Your estimate of the possible present and future (6-10 year) demand for such an SRM in your own operations and elsewhere. (National and international estimates are very useful.)

6. Whether such an SRM, or a similar one, can be produced by, or obtained from a source other than NBS. If so, give reasons to justify its preparation by NBS.

7. Miscellaneous pertinent information to aid justification for the SRM, such as: (a) an estimate of the range of application, monetary significance of the measurement affected, and scientific and/or technological significance including, when feasible, estimates of the impact upon industrial productivity or growth, and (b) supporting letters from industry leaders, trade organizations, interested committees, and others. 


\title{
CERTIFIED REFERENCE MATERIALS FROM OTHER SOURCES
}

Sources of certified reference materials (CRM's) are now world wide. Currently, no international catalog exists, but the International Standards Organization (ISO), through its Council Committee on Reference Materials (REMCO), is in the process of preparing such a catalog. Publication of this international catalog is still in preparation; inquiries should be directed to:

\author{
Mr. M. Parkany \\ Secretary for REMCO \\ International Organization for Standardization \\ 1, Rue de Varembe \\ Case Postale 56 \\ 1211 Geneva 20 \\ Suisse, Switzerland
}

In the period 1972-1977, the International Union of Pure and Applied Chemistry (IUPAC) through its Commission on Physicochemical Measurements and Standards prepared and issued a catalog of CRM's that are useful for the realization of physicochemical properties. The national laboratories that responded to the request for such information were:

\section{COUNTRY}

Australia.

Canada

Germany (West)

Hungary

\section{NATIONAL LABORATORY}

Commonwealth Scientific and Industrial Research Organization

National Standards Laboratory

University Grounds, City Road

Chippendale, NSW 2008

Australia

Canadian Certified Reference Materials Project

C/O Mineral Sciences Laboratories, CANMET

Canada Centre for Mineral and Energy Technology

555 Booth Street

Ottawa, Ontario K1A 0G1 Canada

Bundesanstalt für Materialprüfung

Unter den Eichen 87, D1 Berlin 45

Federal Republic of Germany

The Physikalische-Technische Bundesanstalt Bundesalle 100, 33 Braunschweig

Federal Republic of Germany

National Office of Measures

Németölogyi ut 37-39, sz.

Budapest XII

Hungary 
Japan

.

Standards Department

Agency of Industrial Science and Technology

Ministry of International Trade and Industry

3-1, Kasumigaseki 1, Chiyodaku, Tokyo, Japan

(Cable address: Mitijisc Tokyo)

Netherlands

Institute for Physical Chemistry TNO

Utrechtseweg 48, P.O. Box 108

Zeist

The Netherlands

Poland

Division of Physico-Chemical Metrology

National Board for Quality Control and Measures

2, Elektoralna Street, Warsaw

Poland

United Kingdom

National Physical Laboratory

Teddington, Middlesex

England

The current IUPAC edition is: Physicochemical Measurements: Catalogue of Reference Materials from National Laboratories, Revised 1976, Pure \& Appl. Chem., 48, 503-414 (1976).

Domestic (US) sources, of CRM's, including a large number of uncertified reference materials, are listed in, Guide to United States Reference Materials, NBS Spec. Publ. 260-57 (Feb. 1978), U.S. Government Printing Office, Washington D.C. 20402. 
ACIDIMETRIC

Page

Benzoic acid

Potassium phthalate, acid

ALLOYS CHEMICAL COMPOSITION

(See also individual metals)

Aluminum

Chip form

Solid form

Wire (neutron monitor density)

Cobalt

Wire (neutron monitor density)

Copper

Chip form

Microprobe

Solid form

Ferro (steelmaking)

Gold

High-purity

Microprobe wire

High temperature

Solid form

Iron

Chip and granular form.................................................

Microprobe

Solid form

Lead

Chip form

Solid form

Magnesium

Chip form

Molybdenum

Microprobe

Nickel

Chip form

Oxides

Solid form

Platinum

Doped, wire

High-purity, wire

Selenium Granular form

Silver

Microprobe

Solder

Chip form

Solid form

Tin

Chip form

Titanium

Chip form

Solid form.

Gas-in

Tungsten...

Microprobe

Spelter

Zinc

Chip form

High-purity

Solid form

Zirconium

Chip form

Solid form

ALUMINUM

Al-Co alloy (neutron density monitor wire)

Al-Si alloy.

"Benchmark"

Freezing point standard

Metallo-organic compound

Radioactivity

Wrought alloy

AMERICIUM

Radioactivity
AMMONIUM DIHYDROGEN

PHOSPHATE

Fertilizer

ANALYZED GASES

(ENVIRONMENTAL)

Carbon dioxide in nitrogen

Carbon monoxide in air

Carbon monoxide in nitrogen

Methane in air

Methane-propane in air

Nitric oxide in nitrogen

Oxygen in nitrogen

Propane in air

Sulfur dioxide in nitrogen

\section{ANALYZED LIQUIDS AND SOLIDS}

(ENVIRONMENTAL)

Coal, sulfur in

Coal, trace elements in

Coal fly ash, trace elements in

Fuel oil, trace elements in

Mercury in coal

Mercury in water

Permeation tubes

Sulfur in distillate fuel oil

Sulfur in residual fuel oil

Trace elements in water

ARSENIC

Trioxide (oxidimetric value)

ASSAY STANDARDS

(See specific constituent)

BARIUM

Metallo-organic compound

BASIMETRIC

Tris(hydroxymethl)aminomethane

BENZOIC ACID

Acidimetric.

Calorimetric.

Microchemical

BERYLLIUM ON FILTER MEDIA .............

BIOLOGICAL STANDARDS.

Brewers yeast

Citrus leaves

Liver, bovine

Oyster tissue

Pine needles

Rice flour

Tomato leaves

Tuna, Albacore

Wheat flour

BISMUTH

Radioactivity

BORON

Boric acid

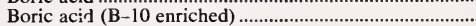

CADMIUM

Metallo-organic compound

Radioactivity

Vapor pressure

CALCIUM

Carbonate (clinical)

Chloride (ion selectivity).

Metallo-organic compound

Molybdate (steelmaking)

CALORIMETRIC .

Combustion calorimetric Heat source 
CANE SUGAR (Sucrose)

CARBIDES

Tungsten carbide.

CARBON

Channel black (rubber compounding)

Conducting black (rubber compounding)

Gas furnace black (rubber compounding)

Oil furnace black (rubber compounding)

Carbon dioxide in nitrogen

Carbon monoxide in nitrogen

CARBON STEELS

CAST IRONS

Blast furnace

Car wheel

Chip form

Ductile

Nodular Solid form

White

CAST STEEL

CELLULAR PLASTIC

CEMENTS

Portland (chemical composition)

Turbidimetric and fineness.

\section{CERAMIC MATERIALS}

\section{Carbides}

Glasses

Minerals

Refractories

CERIUM

Radioactivity

CESIUM

Radioactivity

\section{CHEMICAL STANDARDS}

Primary

Acid potassium phthalate

Arsenic trioxide

Benzoic acid

Boric acid

Potassium chloride

Potassium dichromate

Sodium oxalate

Sucrose

Tris(hydroxymethyl)aminomethane

Uranium oxide

Intermediate purity

Selenium

Zinc....

\section{CHLORINE}

Isotopic reference

Radioactivity

\section{CHROMIUM}

Ferro (steelmaking)

Metallo-organic compound

Isotopic reference

Radioactivity

Refractories

CLAYS

Flint

Plastic

\section{CLINICAL LABORATORY}

STANDARDS

Antiepilepsy drug level assay

Bilirubin

Bovine serum albumin (powder)

Bovine serum albumin (solution)

Calcium carbonate

Cholesterol

Creatinine

Cortisol
Gallium melting point

Glass filters for spectrophotometry

D-Glucose

Iron metal

Human serum

Lead nitrate

Liquid filters for spectrophotometry

Lithium carbonate

Magnesium gluconate

D-Mannitol

Metals on quartz filters

Potassium chloride

Potassium dichromate

Quartz cuvette for spectrophotometry

Quinine sulfate dihydrate

Sodium chloride

Sodium pyruvate

Thermometer, enzymology

Tris(hydroxymethyl)aminomethane

Tris(hydroxymethyl)aminomethane $\mathrm{HCL}$

Urea

Uric acid

VMA

COAL

Fly ash, trace elements in

Mercury in

Sulfur in

Trace elements in

COATING THICKNESS

Copper on steel

Gold on copper

Gold on copper-clad glass-epoxy laminate

Gold on Fe-Ni-Co glass sealing a!loy

Gold on nickel

Nickel on brass

Nickel on steel

Tin on steel

\section{COBALT}

Metallo-organic compound

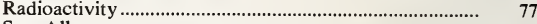

See, Alloys

COLOR STANDARDS

\section{COPPER}

"Benchmarks" (chips).

"Benchmarks" (solids).

Beryllium copper alloys

Brass

Aluminum

Cartridge.......

Free cutting

Naval

Red

Sheet

Bronze

Commercial

Leaded tin

Silicon

Coating thickness

Concentrate.

Cupro-nickel (chips)

Cupro-nickel (solids).

Freezing point

Guilding metal

Isotopic reference

Linear thermal expansion.

Metallo-organic compound

Microprobe (gold-copper wires) ............................................. $\quad 42$

Mill heads

Mill tails

Nickel silver alloy

\section{DENSITY STANDARDS}

Neutron density wire

Photographic.

Smoke density, flaming 
Smoke density, non-flaming.

2,2,4-trimethylpentane

$\mathrm{X}$-ray.......

DEXTROSE (Glucose)

DIELECTRIC

Cyclohexane

1,2-dichloroethane

Nitrobenzene

DIFFERENTIAL THERMAL ANALYSIS.

ENVIRONMENTAL STANDARDS

Gases

Liquids

Radioactivity

Solids.

ELASTICITY

ELECTRICAL RESISTIVITY

FELDSPARS

Soda

Potash

FERRO-(STEELMAKING ALLOYS) ..........

Chromium

Niobium

Phosphorus

Silicon

FERTILIZER

Ammonium dihydrogen phosphate

Phosphate rock

Potassium dihydrogen phosphate

Potassium nitrate.

FILTERS

Glass for spectrophotometry

Liquid for spectrophotometry

Metal-on-Quartz

FISSION TRACK GLASSES

FLAMMABILITY STANDARDS

Flame spread index (hardboard sheet)

Smoke density, flaming

Smoke density, non-flaming

See also, Special Reference Materials

FLUORINE IN URINE

FLUORSPAR

Assay

Geological

FORENSIC STANDARDS

Glass, refractive index

Silicone liquids, refractive index

FREEZE DRIED URINE

Fluorine

Mercury

FREEZING POINT STANDARDS

Defining fixed points

Tin

Zinc

Determined reference points

Aluminum

Copper

Lead

Mercury

Tin

Zinc

FUELS (see, environmental)

Isooctane

n-Heptane

GALLIUM

Melting point.

GAS TRANSMISSION

Polyester plastic film for oxygen

GASES, ANALYZED.

Carbon Dioxide in nitrogen

Carbon monoxide in air
Carbon monoxide in nitrogen

Methane in air

Methane-propane in air

Nitric oxide in nitrogen

Nitrogen dioxide permeation device

Oxygen in nitrogen

Propane in air

Sulfur dioxide in nitrogen

Sulfur dioxide permeation tubes

GASES IN METALS

"GASOLINE"'

Lead in

Fuel ratings.

GEOLOGICAL

Fluorspar

\section{GLASS}

Chemical composition .

Filters for spectrophotometry

Fission track.

Physical properties..

Refractive index

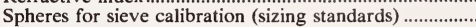

Stress-optical coefficient........................................................

Trace elements in.

GLUCOSE (Dextrose)

Clinical.

Primary chemical.

\section{GOLD}

Coating thickness

on epoxy

on $\mathrm{Fe}-\mathrm{Ni}$-Co glass sealing alloy

on nickel

High-purity

Microprobe.

Vapor pressure...

HEAT STANDARDS

Calometric

Freezing points

Melting points

Superconductive fixed points

Thermal conductivity

Thermal expansion

Thermocouple materials

Vapor pressure

HEAT SOURCE

Zirconium-barium-chromate

HIGH-PURITY METALS

Gold, wire and rod

Platinum, wire

Zinc

HIGH TEMPERATURE ALLOYS ................

HYDROGEN

in steel (GM-1, GM-2)

in titanium...

Radioactivity

INDUSTRIAL HYGIENE

Beryllium/filter

Fluorine in urine

Mercury in urine

Metals/filter

Quartz/filter

ION-ACTIVITY

Ion-selective electrodes

pD standards

pH standards

IRON

Chip form

Clinical.

Electrical resistivity....

Metallo-organic compound.

Ores...

Radioactivity 
Solid form

Thermal conductivity

IODINE

Radioactivity

ISOTOPIC REFERENCE STANDARDS ....

Boron, natural

Boron, enriched

Bromine

Chlorine

Chromium

Copper

Lead, equal atom (206/208)

Lead, natural

Lead, radiogenic, $92 \%$ lead-206

Lead-206, spike

Magnesium

Plutonium

Rhenium

Rubidium

Silicon

Silver

Strontium

Uranium-233 spike

Uranium-235, spike

KRYPTON

Radioactivity

LEAD

Alloys

Bearing metal

Freezing point

Gasoline

Isotopic reference

Nitrate, clinical.

Solder

LIGHT SENSITIVE STANDARDS

Faded paper strips

Light sensitive papers

Light sensitive plastic chips

LIMESTONE.

Argillaceous

Dolomitic

LINERBOARD FOR TAPE ADHESION

TESTING

LIQUIDS, ANALYZED

Lead in gasoline.

Sulfur in residual fuel oil

Sulfur in distillate fuel oil..

LITHIUM

Metallo-organic compound

Ores.........................

LOW ALLOY STEELS

Chip form

Solid form

MAGNESIUM

Alloy

Gluconate, clinical

Isotopic reference

Metallo-organic compound

MAGNETIC

Susceptibility.

Tape, computer amplitude reference

MAGNIFICATION STANDARD ...............

MANGANESE

Fluoride, magnetic susceptibility

Metallo-organic compound

MARAGING STEEL

MERCURY

Freezing point

Radioactivity

Trace element in coal
Trace element in urine

Trace element in water.

MELTING POINTS

Alumina

Gallium

METALLO-ORGANIC COMPOUNDS

Aluminum

Barium

Cadmium

Calcium

Chromium

Cobalt

Copper

Iron

Lithium

Magnesium

Manganese

Nickel

Phosphorus

Silicon

Silver

Sodium

Strontium

Tin

Vanadium

Zinc

METALLURGICAL STANDARDS

Austenite in ferrite

Iron carbide in ferrite

METALS

Alloys-see index entry

Elements-see index entry

Freezing points.

Gas-in.

High-purity

Melting points

Microprobe.

Resistivity, electrical

Thermal conductivity

Vapor pressure

METALS IN OIL, WEAR

METALS ON FILTER MEDIA

Beryllium

Cadmium

Lead

Manganese

Zinc

MICROCHEMICAL STANDARDS

Acetanilide

Anisic acid

Benzoic acid

o-Bromobenzoic acid

m-Chlorobenzoic acid

Cystine

p-Fluorobenzoic acid

Nicotinic acid

Urea

MICROCOPY RESOLUTION TEST

CHARTS

MICROPROBE STANDARDS

Cartridge brass

Glasses for microanalysis

Gold-copper

Gold-silver

Iron-3\% silicon

Tungsten-20\% molybdenum 
MINERALS.

Clays

Flint

Plastic

Feldspar

Potash

Soda

Limestone

Argillaceous

Dolomitic

Ores

Bauxite

Copper

Fluorspar

Iron

Lithium

Molybdenum

Phosphate rock

Tungsten

Zinc

MOLECULAR WEIGHT

Polyethylene

Polystyrene

MOLYBDENUM

Concentrate (ore)

Heat capacity

Microprobe

MOSSBAUER STANDARDS

NEUTRON DENSITY MONITOR WIRE

Cobalt in aluminum

NICKEL

Alloys

Chip form

Solid form

Coating thickness

on brass

on steel

Metallo-organic compound

Oxides

Radioactivity.

NIOBIUM

Radioactivity

NITRIC OXIDE

In nitrogen

NITROGEN IN

Cast iron

Ingot iron

Steel

Titanium

Zirconium

NONFERROUS ALLOYS

NUCLEAR MATERIALS

Neutron density monitor wire

Plutonium assay

Plutonium isotopic

Uranium assay

Uranium isotopic

OIL

Sulfur in.

Trace elements in

ORES

Bauxite

Copper

Fluorspar

Iron

Lithium

Molybdenum

Phosphate rock

Tungsten

Zinc
OXALIC ACID

Radioactivity......

OXIDIMETRIC

Arsenic trioxide

Potassium dichromate

Sodium oxalate

OXIDES

Iron

Nitrogen.

Titanium.

Uranium.

Zinc (rubber compounding).

OXYGEN IN

Ingot iron

Nitrogen

Steel

Titanium

Zirconium

PAINT, LEAD-BASED ………………...... 48

PAPER

Faded strips

Light sensitive

PARTICULATE, URBAN

PERMEATION TUBES

Nitrogen dioxide

Sulfur dioxide

PERMITTIVITY

pD STANDARDS

Disodium hydrogen phosphate

Potassium dihydrogen phosphate

Sodium bicarbonate

Sodium carbonate

pH STANDARDS

Acid potassium phthalate

Borax

Disodium hydrogen phosphate

Potassium dihydrogen phosphate

Potassium hydrogen tartrate

Potassium tetroxalate

Sodium bicarbonate

Sodium carbonate

Tris(hydroxymethyl)aminomethane

Tris(hydroxymethyl)aminomethane $\mathrm{HCl}$

PHOSPHATE

Ammonium dihydrogen

Potassium dihydrogen

See, $\mathrm{pH}$ standards

Rock

PHOSPHORS

PHOSPHORUS

Ferro (steelmaking alloy)

Metallo-organic compound

PHOTOGRAPHIC

Microcopy resolution test chart

Step tablets

PLASTIC (See, Polymer)

90

Polyester film for oxygen transmission..................................

PLATINUM

Doped, wire

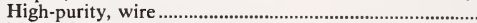

Magnetic susceptibility

Thermoelement

PLUTONIUM

Metal assay

Standard matrix

Sulfate tetrahydrate 
POLYMER

Molecular weight.

Oxygen transmission

Permittivity

POLYSTYRENE

POTASSIUM

Acid phthalate.

Chloride (clinical)

Dichromate (clinical)

Dichromate (oxidimetric)

Iodide (spectrophotometric)

See, Fertilizer

See, Ion activity

POWDER DIFFRACTION STANDARDS

$\alpha$-alumina

Cerium oxide

Chromium oxide

Rutile

Silicon

Zinc oxide

PRIMARY CHEMICALS

Arsenic trioxide

Benzoic acid

Boron

Dextrose

Plutonium metal

Plutonium sulfate tetrahydrate

Potassium chloride

Potassium dichromate

Potassium acid phthalate

Rubidium chloride

Sodium oxalate

Strontium carbonate

Sucrose

Tris(hydroxymethyl)aminomethane

Uranium metal

Uranium oxide

PROMETHIUM

Radioactivity

PROPANE

QUARTZ

$\alpha$ - for HF solution calolimetry

Cuvette for spectrophotometry

Filter media, on...

RADIOACTIVITY STANDARDS

Alpha-particle

Beta-particle and gamma ray gas

Beta-particle, gamma-ray, and electron-capture solutions

Contemporary standard for carbon-14 dating

laboratories

Environmental

Gamma-ray "point-sources"

Mixed radionuclides

Radium gamma-ray solutions

Radium solutions for radon analysis

RADIUM

Radioactivity

RADON

Radioactivity

REFRACTIVE INDEX

Glass

Silicone liquids

2,2,4-Trimethylpentane

Toluene

REFRACTORY MATERIALS

RESEARCH MATERIALS

Aluminum ultra purity

Copper heat capacity

Glass for microanalysis

Phosphors

River sediment

Tuna, Albacore

RESISTIVITY

Electrical

Silicon

RHENIUM

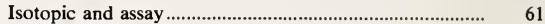

RUBBER ........................................................... 84

RUBBER COMPOUNDING ....................... 84

RUBIDIUM

Isotopic

SAPPHIRE

Enthalpy and heat capacity ........................................... 72

Thermal expansion.............................................................. 73

SEDIMENT

Estuarine

River, environmental

River, radioactivity.

SELENIUM

Metal

Radioactivity

SILICON

Ferro (steelmaking alloy)

Isotopic

Metallo-organic compound

Refractories $\left(\mathrm{SiO}_{2}\right)$

Resistivity.

$\mathrm{X}$-ray diffraction, powder

SILICONE LIQUIDS (refractive index)......... 77

SILVER

Alloys (microprobe)

Isotopic reference.

Metallo-organic compound

SIZING STANDARDS

Calibrated glass spheres

Turbidimetric and fineness (cement)

\section{SMOKE DENSITY CHAMBER}

STANDARDS

SODIUM

Bromide (isotopic) .

Metallo-organic compound

Oxalate

Radioactivity

See, Ion-activity

SOLDER

Chip form

Solid form

SOLIDS, ANALYZED

48

SPECTROPHOTOMETRIC STANDARDS 75

Didymium-oxide glass filters

Glass filters

Liquid filters

Metal on quartz filters

Potassium dichromate

Potassium iodide (stray light)

Quartz cuvette

Quinine sulfate dihyudrate

STAINLESS STEEL

Chip form .

Resistivity

Solid form 
STEELMAKING ALLOYS

Calcium molybdate

Ferrochromium

Ferroniobium

Ferrophosphorus

Ferrosilicon

Silicone, refined

STEEL, THERMAL CONDUCTIVITY.......

STEELS, CAST

STEELS, CHIP FORM

High alloy

Low alloy

Low alloy, special

Plain carbon

Stainless

Tool

STEELS, GAS-IN

STEELS,

GRANULAR FORM.

STEELS, SOLID FORM

Cast

High temperature alloy

Low alloy

Low alloy, special

Maraging Oxygen

Stainless

Toll

STEP TABLETS

Photographic

$\mathrm{X}$-ray

STRONTIUM

Isotopic reference

Metallo-organic compound

Radioactivity

SUCROSE

Primary chemical

SULFUR

Elemental (rubber compounding)

in residual fuel oils.

in coal....

SUPERCONDUCTIVE THERMOMETRIC

FIXED POINT DEVICES

SURFACE FLAMMABILITY

TAPE, MAGNETIC-SECONDARY

REFERENCE

Cartridge

Cassette

Reel

TEMPERATURE (See, Heat

STANDARDS)

Calorimetry

Differential thermal analysis

Heat source.

THERMAL CONDUCTIVITY.

THERMAL EXPANSION

THERMOCOUPLE MATERIALS

THERMOMETERS

THORIUM

Radioactivity

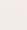

\section{TURBIDIMETRIC AND FINENESS \\ STANDARD}

URANIUM

Isotopic and assay

Metal

Oxide

Depleted

Enriched

Primary standard

\section{VANDIUM}

Metallo-organic compound

VAPOR PRESSURE STANDARD

VISCOSITY STANDARDS

Glass..

WATER, TRACE ELEMENTS IN

WEAR METALS IN OIL

X-RAY STEP TABLET

X-RAY DIFFRACTION

Silicon powder

YTTRIUM

Radioactivity

ZINC

Chip form

Concentrate, ore

71

Freezing point

High purity

Intermediate purity

Metallo-organic compound........................

Oxide (rubber compounding)

Solid form

Spelter modified

ZIRCONIUM

Chip form

Solid form 


\section{NUMERICAL INDEX TO SRM CERTIFICATES}

The Certificate Date listed is the current version of the Certificate. Those dates followed by the letter "P" indicate that it is a Provisional Certificate.

In general, Provisional Certificates are issued for Standard Reference Materials before all of the values have been certified, but after a sufficient number of values are certified so that the material is a valuable standard for the intended purpose. As additional values are certified, the Provisional Certificate may be revised and when and if all of the values are certified, the final Certificate is issued.

When new or revised Certificates are issued for SRM's they are announced in the SRM Price and Availability List which supplements this catalog. SRM purchasers whose Certificate shows an earlier date than listed below may obtain the current version of the Certificate from the Office of Standard Reference Materials, Room B311, Chemistry Building, National Bureau of Standards, Washington, D.C. 20234.

One or more of the following letters apply to materials where no date is listed.
A. Individually Certified.
B. The Material is issued with "Instructions for Use" in lieu of a Certificate.
C. This Material is not certified, refer to page reference for details.
D. Material is in preparation.
E. Research Material: Issued with a "Report of Investigation."
F. Special Reference Material: Information provided, but not certified by NBS.
G. Set of SRM's: Issued with Certificates for the individual SRM's.
$\mathrm{H}$. Not individually issued, part of a set. 


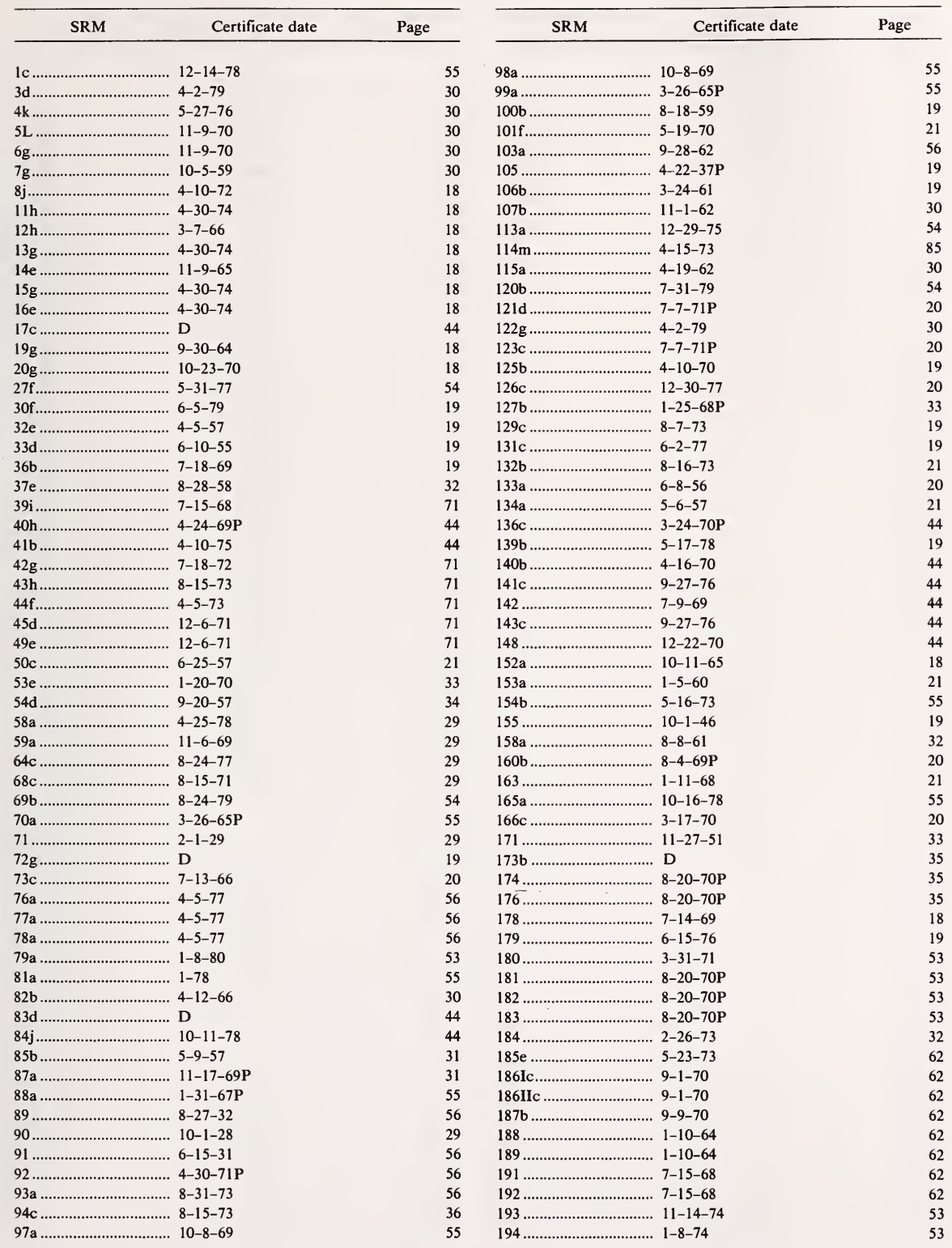




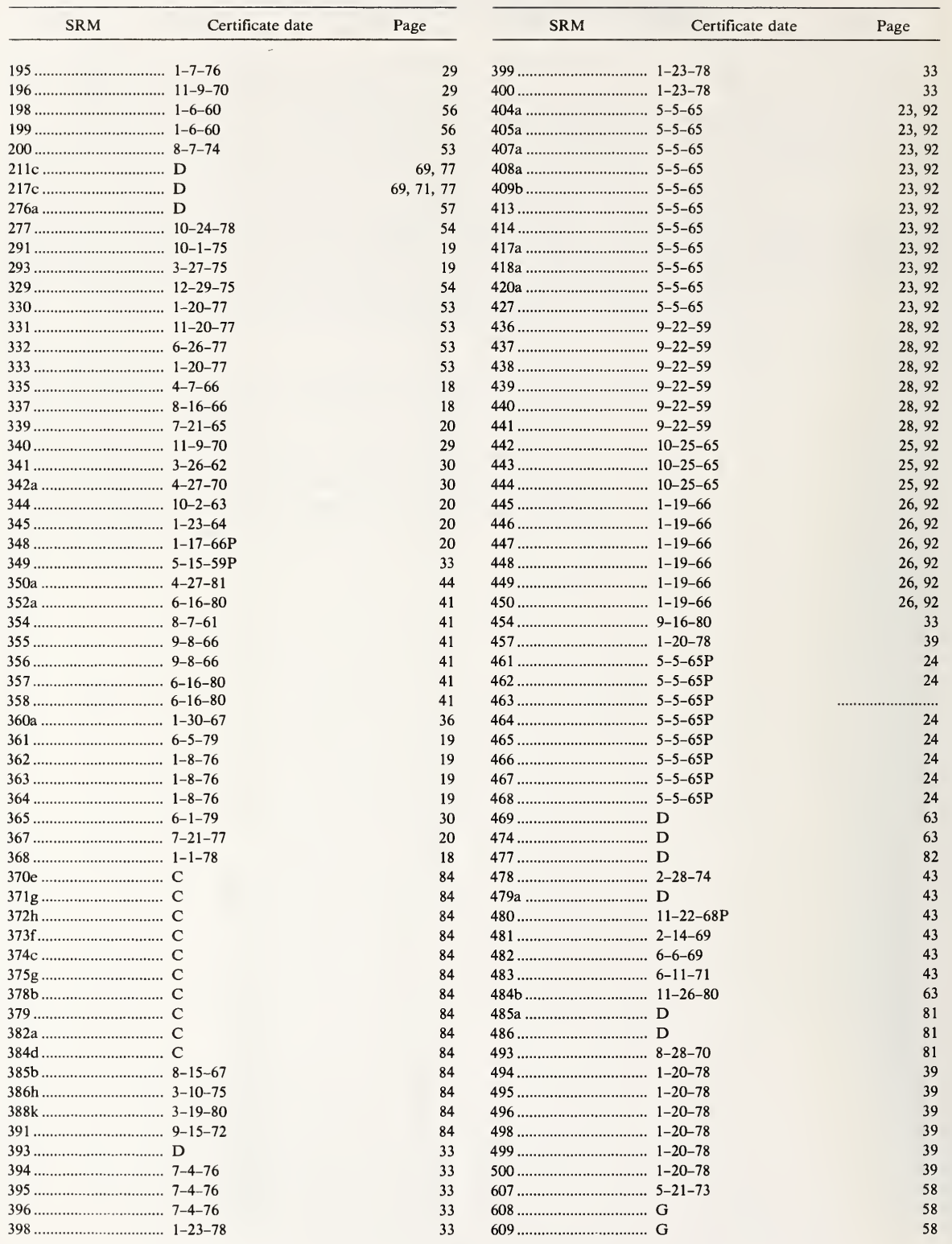




\begin{tabular}{|c|c|c|c|}
\hline Certificate date & Page & Certificate date & Page \\
\hline 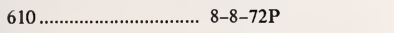 & 58 & 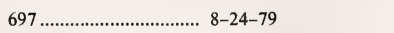 & 54 \\
\hline 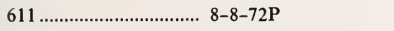 & 58 & 698 & 54 \\
\hline 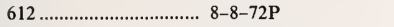 & 58 & $700 \mathrm{~d}$ & 86 \\
\hline $613 \ldots \ldots \ldots-12 \mathrm{P}$ & 58 & 701d ....................... 1-15-77 & 86 \\
\hline 614 & 58 & 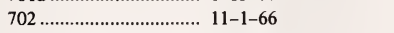 & 86 \\
\hline 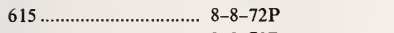 & 58 & 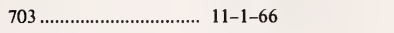 & 86 \\
\hline 616 & 58 & 705 & 69,72 \\
\hline 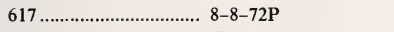 & 58 & 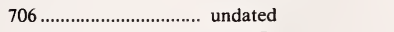 & 69 \\
\hline 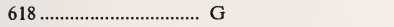 & 58 & 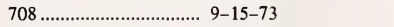 & 67 \\
\hline 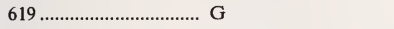 & 58 & 709 & 67,68 \\
\hline 620 & 56 & 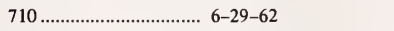 & 67,68 \\
\hline 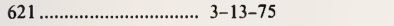 & 56 & 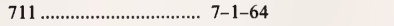 & 67,68 \\
\hline $622 \ldots \ldots \ldots \ldots+3-19-76$ & 67 & 712 & 67,68 \\
\hline 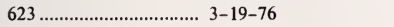 & 67 & 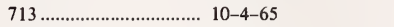 & 67,68 \\
\hline 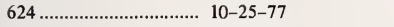 & 67 & 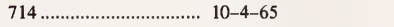 & 67,68 \\
\hline 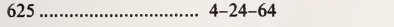 & 40 & $715 \ldots \ldots \ldots \ldots$ & 67,68 \\
\hline 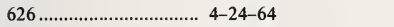 & 40 & 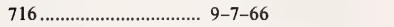 & 67,68 \\
\hline 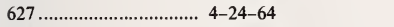 & 40 & 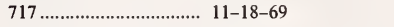 & $67,68,69$ \\
\hline $628 \ldots \ldots \ldots \ldots \ldots \ldots \ldots \ldots \ldots \ldots \ldots \ldots \ldots . . .14-24-64$ & 40 & $718 \ldots \ldots \ldots \ldots \ldots \ldots \ldots \ldots \ldots \ldots \ldots \ldots \ldots \ldots$ & 69 \\
\hline 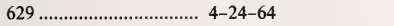 & 40 & 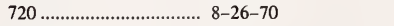 & 72 \\
\hline 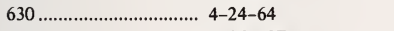 & 40 & $723 \mathrm{a}$ & 44,67 \\
\hline $631 \ldots \ldots \ldots \ldots \ldots \ldots-16-70 \mathrm{P}$ & 40 & $724 a$ & 72 \\
\hline 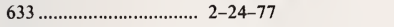 & 57 & 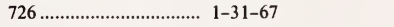 & 34 \\
\hline $634 \ldots \ldots \ldots \ldots \ldots$ & 57 & 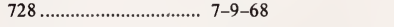 & 36 \\
\hline 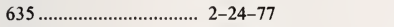 & 57 & 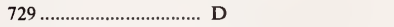 & 72 \\
\hline 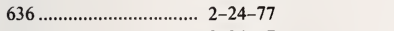 & 57 & 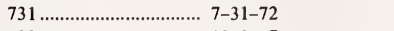 & 73 \\
\hline 637 & 57 & 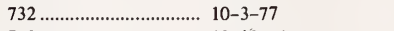 & 73 \\
\hline 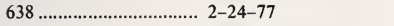 & 57 & 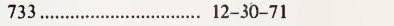 & 74 \\
\hline 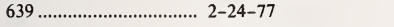 & 57 & 736a ........................... D & 73 \\
\hline 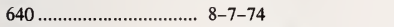 & 82 & 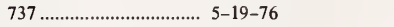 & 73 \\
\hline 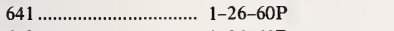 & 40 & 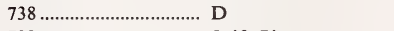 & 73 \\
\hline 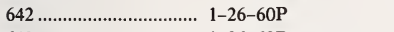 & 40 & 739 & 73 \\
\hline 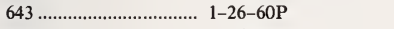 & 40 & $740 \ldots \ldots \ldots \ldots \ldots \ldots \ldots \ldots \ldots \ldots \ldots \ldots \ldots \ldots \ldots . . .2-19-70$ & 71 \\
\hline 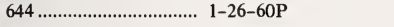 & 40 & 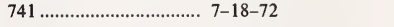 & 71 \\
\hline 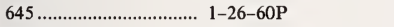 & 40 & 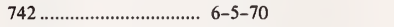 & 71 \\
\hline $646 \ldots \ldots \ldots \cdots \cdots \cdots \cdots \cdots \cdots \cdots \cdots \cdots \cdots \cdots \cdots \cdots \cdots$ & 40 & 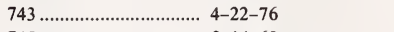 & 71 \\
\hline 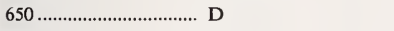 & 35 & 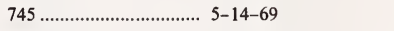 & 72 \\
\hline 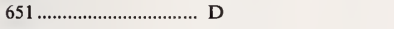 & 35 & 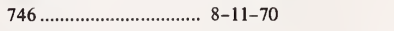 & 72 \\
\hline 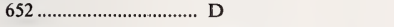 & 35 & 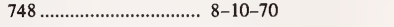 & 72 \\
\hline 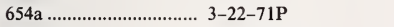 & 40 & 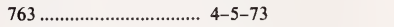 & 74 \\
\hline $661 \ldots \ldots \ldots \ldots \ldots \ldots \ldots \ldots \ldots \ldots+\ldots$ & 24 & 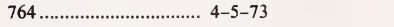 & 74 \\
\hline $662 \ldots \ldots \ldots \ldots \ldots \ldots \ldots+18-72 \mathrm{H})$ & 24 & 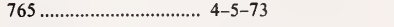 & 74 \\
\hline 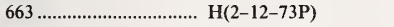 & 24 & 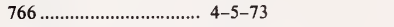 & 74 \\
\hline 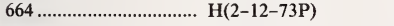 & 24 & 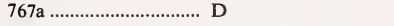 & 70 \\
\hline 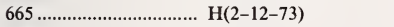 & 24 & 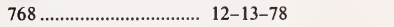 & 70 \\
\hline 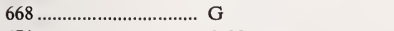 & 24 & 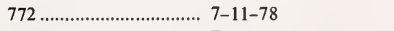 & 74 \\
\hline 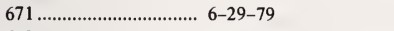 & 34 & 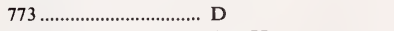 & 68 \\
\hline 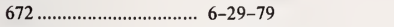 & 34 & 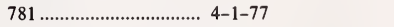 & 72 \\
\hline 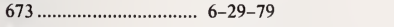 & 34 & $782 \ldots \ldots \ldots \ldots \ldots \ldots \ldots \ldots \ldots \ldots \ldots \ldots \ldots \ldots \ldots \ldots \ldots$ & 72 \\
\hline 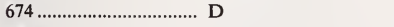 & 82 & 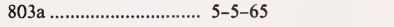 & 92 \\
\hline 680a _......................... 3-1-77 & 42 & D803a ........................... 5-5-65 & 92 \\
\hline 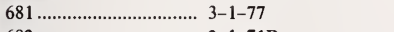 & 42 & 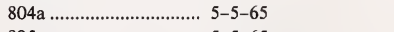 & 92 \\
\hline 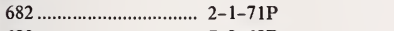 & 42 & $805 a \ldots \ldots \ldots \ldots \ldots \ldots \ldots \ldots \ldots \ldots \ldots \ldots \ldots \ldots \ldots . . .5-5-65$ & 92 \\
\hline 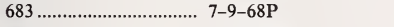 & 42 & 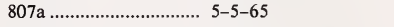 & 92 \\
\hline 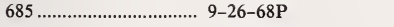 & 42 & D807a ........................... 5-5-65 & 92 \\
\hline $690 \ldots \ldots \ldots \ldots \ldots \ldots \ldots \ldots \ldots \ldots \ldots \ldots \ldots \ldots \ldots$ & 54 & 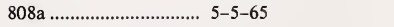 & 92 \\
\hline 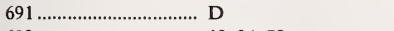 & 54 & 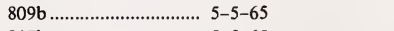 & ....... \\
\hline 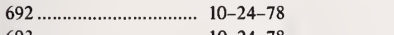 & 54 & 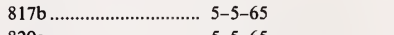 & 92 \\
\hline 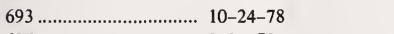 & 54 & 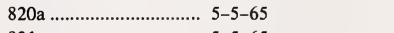 & 92 \\
\hline 696 ………................ 8-24-79 & 54 & 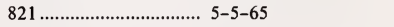 & 92 \\
\hline
\end{tabular}




\begin{tabular}{|c|c|c|c|}
\hline Certificate date & Page & Certificate date & Page \\
\hline 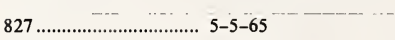 & 92 & 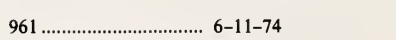 & 60 \\
\hline 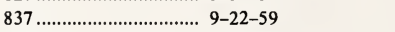 & 28,92 & 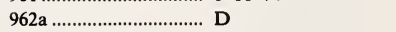 & 60 \\
\hline D837 ........................... 9-22-59 & 28,92 & 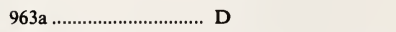 & 60 \\
\hline 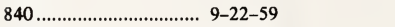 & 28,92 & 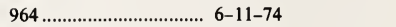 & 60 \\
\hline D840 ........................... 9-22-59 & 28,92 & 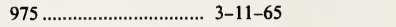 & 61 \\
\hline D841 …................... 9-22-59 & 28,92 & 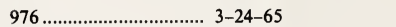 & 61 \\
\hline 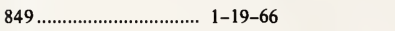 & 92 & 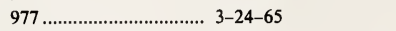 & 61 \\
\hline 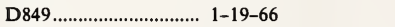 & 92 & 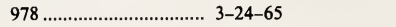 & 61 \\
\hline 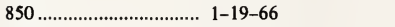 & 92 & 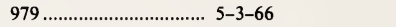 & 61 \\
\hline 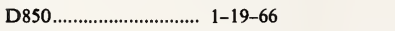 & 92 & 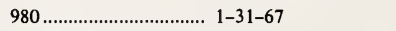 & 61 \\
\hline $871 \ldots$ & 32 & $981-3 \ldots \ldots \ldots \ldots \ldots \ldots \ldots \ldots .10-13$ & 61 \\
\hline 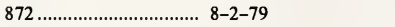 & 32 & 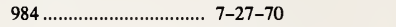 & 44,61 \\
\hline 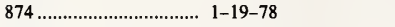 & 32 & 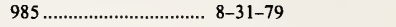 & 44 \\
\hline 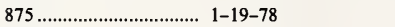 & 32 & $987 \ldots \ldots \ldots \ldots \ldots \ldots \ldots \ldots+3-6-72 P$ & 44 \\
\hline 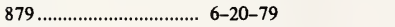 & 32 & 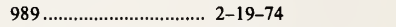 & 61 \\
\hline 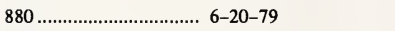 & 32 & 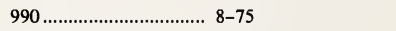 & 61 \\
\hline 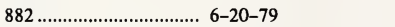 & 33 & 991 & 61 \\
\hline 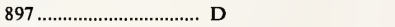 & 34 & 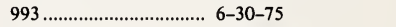 & 59,61 \\
\hline 898 & 34 & 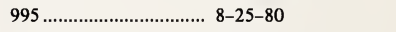 & 59,61 \\
\hline 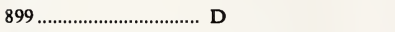 & 34 & 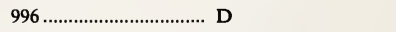 & 59 \\
\hline 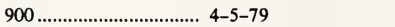 & 45 & $999 \ldots \ldots \ldots \ldots \ldots \ldots \ldots \ldots \ldots \ldots . . . . .19-6-72$ & 44 \\
\hline 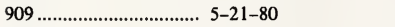 & 45 & $1001 \ldots \ldots \ldots \ldots \ldots \ldots \ldots \ldots \ldots \ldots \ldots \ldots$ & 87 \\
\hline 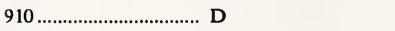 & 45 & 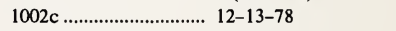 & 87 \\
\hline 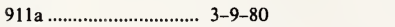 & 45 & 1003 & 85 \\
\hline 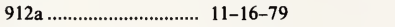 & 45 & 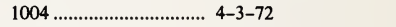 & 85 \\
\hline 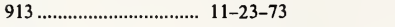 & 45 & $1006 a$ & 87 \\
\hline 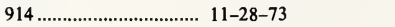 & 45 & 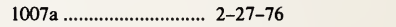 & 87 \\
\hline 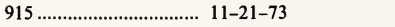 & 45 & 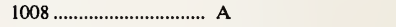 & 87 \\
\hline $916 \ldots \ldots \ldots \ldots \ldots \ldots \ldots \ldots \ldots . . . . .13-10-71$ & 45 & 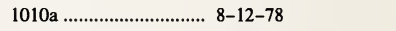 & 87 \\
\hline 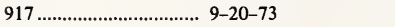 & 45 & 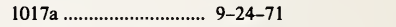 & 85 \\
\hline 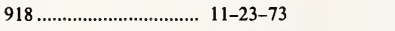 & 45 & 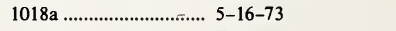 & 85 \\
\hline 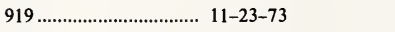 & 45 & 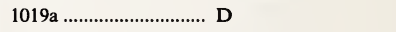 & 85 \\
\hline 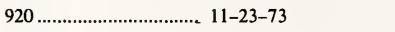 & 45 & 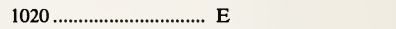 & 90,92 \\
\hline 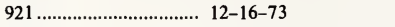 & 45 & $1021 \ldots \ldots \ldots \ldots \ldots \ldots \ldots \ldots \ldots \ldots$ & 90,92 \\
\hline 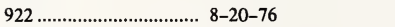 & 45,62 & $1022 \ldots \ldots \ldots \ldots \ldots \ldots \ldots \ldots \ldots \ldots$ & 90,92 \\
\hline 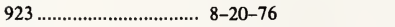 & 45,62 & $1023 \ldots \ldots \ldots \ldots \ldots \ldots \ldots \ldots \ldots \ldots$ & 90,92 \\
\hline 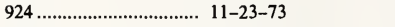 & 45 & $1024 \ldots \ldots \ldots \ldots \ldots \ldots \ldots \ldots \ldots \ldots \ldots$ & 90,92 \\
\hline 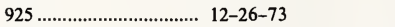 & 45 & 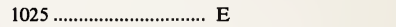 & 90,92 \\
\hline 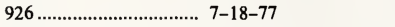 & 45 & $1026 \ldots \ldots \ldots \ldots \ldots \ldots \ldots \ldots \ldots \ldots$ & 90,92 \\
\hline $927 \ldots \ldots \ldots \ldots \ldots \ldots \ldots \ldots \ldots \ldots \ldots . . .18-18$ & 45 & 1027 & 90,92 \\
\hline 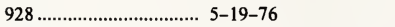 & 45 & 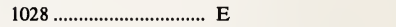 & 90,92 \\
\hline 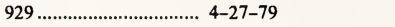 & 45 & $1029 \ldots \ldots \ldots \ldots \ldots \ldots \ldots \ldots$ & 90,92 \\
\hline 930D & 45,76 & $1030 \ldots \ldots \ldots$ & 90,92 \\
\hline 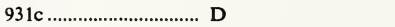 & 45,76 & $1031 \ldots \ldots \ldots \ldots \ldots \ldots \ldots \ldots \ldots$ & 90,92 \\
\hline $932 \ldots \ldots \ldots \ldots \ldots \ldots \ldots \ldots \ldots \ldots \ldots$ & 45,76 & $1032 \ldots \ldots \ldots \ldots \ldots \ldots \ldots \ldots \ldots \ldots$ & 90,92 \\
\hline 934 & 45 & 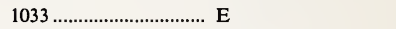 & 90,92 \\
\hline 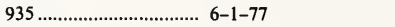 & 45,76 & 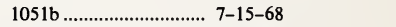 & 52 \\
\hline 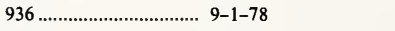 & 45,76 & $1052 \mathrm{~b}$ & 52 \\
\hline 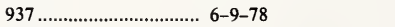 & 45 & 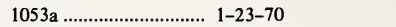 & 52 \\
\hline 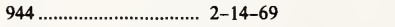 & 44,59 & $1055 b$ & 52 \\
\hline $945 \ldots \ldots \ldots \ldots \ldots \ldots \ldots \ldots \ldots \ldots \ldots \ldots$ & 59 & 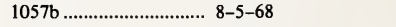 & 52 \\
\hline $946 \ldots \ldots \ldots \ldots \ldots \ldots \ldots \ldots \ldots \ldots$ & 59 & 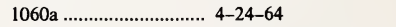 & 52 \\
\hline 947 & 59 & $1061 \mathrm{c} \ldots \ldots \ldots \ldots \ldots \ldots . . .1 . .19-9-70 \mathrm{P}$ & 52 \\
\hline $948 \ldots \ldots \ldots \ldots \ldots \ldots \ldots . . .19-1-72 P$ & 59 & 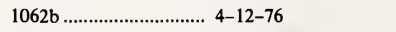 & 52 \\
\hline $949 \mathrm{e}$ & 44,59 & 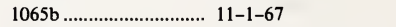 & 52 \\
\hline 950b ........................ 3-1-78 & 44,59 & $1066 a$ & 52 \\
\hline 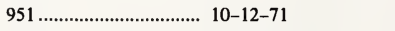 & 44,61 & $1069 \mathrm{~b}$ & 52 \\
\hline 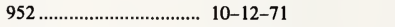 & 61 & 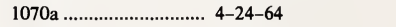 & 52 \\
\hline 953 & 59,60 & 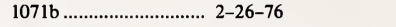 & 52 \\
\hline $960 \ldots \ldots \ldots \ldots \ldots \ldots \ldots \ldots$ & 44 & 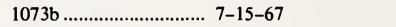 & 52 \\
\hline
\end{tabular}




\begin{tabular}{|c|c|c|c|}
\hline Certificate date & Page & Certificate date & Page \\
\hline 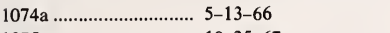 & 52 & 1123 & 37 \\
\hline $1075 \mathrm{a}$ & 52 & $\mathrm{C} 1123 \ldots \ldots+12-19-66 \mathrm{P}$ & 37 \\
\hline 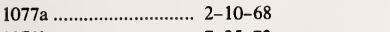 & 52 & 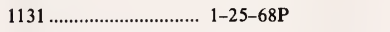 & 39 \\
\hline $1078 \mathrm{~b}$ & 52 & 1132 & 39 \\
\hline 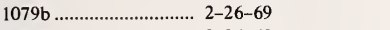 & 52 & 1134 & 24 \\
\hline $1080 a \ldots \ldots \ldots \ldots \ldots \ldots . . .2-26-69$ & 52 & 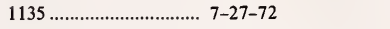 & 24 \\
\hline 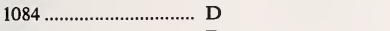 & 53 & 1136 & 24 \\
\hline 1085 & 53 & 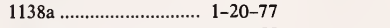 & 31 \\
\hline 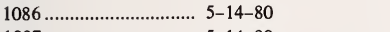 & 41 & 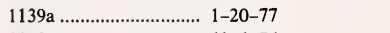 & 31 \\
\hline 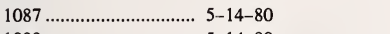 & 41 & 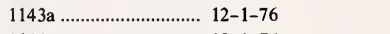 & 31 \\
\hline 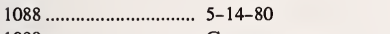 & 41 & 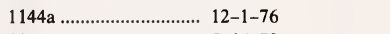 & 31 \\
\hline 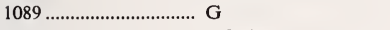 & 41 & 1145 & 31 \\
\hline $1090 \ldots \ldots \ldots \ldots \ldots \ldots \ldots \ldots \ldots \ldots \ldots \ldots \ldots \ldots . . .4-2-69$ & 41 & $1146 \ldots \ldots \ldots \ldots \ldots \ldots \ldots \ldots \ldots \ldots \ldots . . .5-16-78$ & 31 \\
\hline 1091 & 41 & 1150 & 31 \\
\hline $1092 \ldots \ldots \ldots \ldots \ldots \ldots \ldots \ldots \ldots . . .4-2-69$ & 41 & 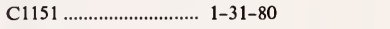 & 26 \\
\hline 1093 & 41 & 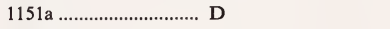 & 26 \\
\hline $1094 \ldots \ldots \ldots \ldots \ldots . .12-69 P$ & 41 & 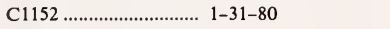 & 26 \\
\hline 1095 & 41 & $1152 \mathrm{a}$ & 26 \\
\hline $1096 \ldots \ldots \ldots \ldots . . .16-13-72)$ & 41 & $\mathrm{C} 1153 \ldots \ldots \ldots \ldots \ldots \ldots \ldots \ldots \ldots . . .1-31-80$ & 26 \\
\hline $1097 \ldots \ldots \ldots \ldots \ldots . . \mathrm{H}(5-26-72 \mathrm{P})$ & 41 & 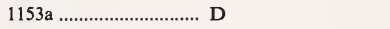 & 26 \\
\hline $1098 \ldots \ldots \ldots \ldots \ldots . . . \mathrm{H}(10-3-73)$ & 41 & $\mathrm{C} 1154 \ldots \ldots \ldots \ldots \ldots \ldots \ldots \ldots . . . .1-31-80$ & 26 \\
\hline 1099 & 41 & 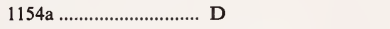 & 26 \\
\hline $\mathrm{C} 1101 \ldots \ldots \ldots \ldots \ldots \ldots \ldots \ldots \ldots \ldots \ldots . . .7-31-64 \mathrm{P}$ & 37 & 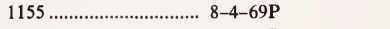 & 26 \\
\hline $1102 \ldots \ldots \ldots \ldots \ldots \ldots \ldots \ldots \ldots . .7-31-64 P$ & 37 & $1156 \ldots \ldots \ldots \ldots \ldots \ldots \ldots . . .4-5-66 \mathrm{P}$ & 27 \\
\hline 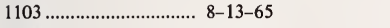 & 37 & 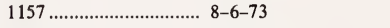 & 28 \\
\hline 1104 & 37 & 1158 & 27 \\
\hline 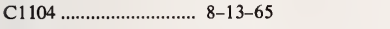 & 37 & 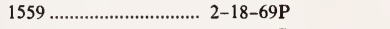 & 40 \\
\hline $\mathrm{C} 1105 \ldots \ldots \ldots \ldots \ldots . . .8-13-65$ & 37 & $1160 \ldots \ldots \ldots \ldots \ldots . .2-18-69 \mathrm{P}$ & 40 \\
\hline $1106 \ldots \ldots \ldots \ldots \ldots \ldots \ldots \ldots \ldots \ldots . . . .11-17-69 \mathrm{P}$ & 37 & 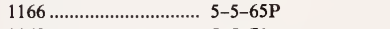 & 24 \\
\hline $\mathrm{C} 1106$ & 37 & 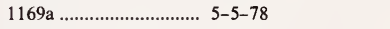 & 24 \\
\hline 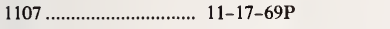 & 37 & $1170 \mathrm{~b} \ldots \ldots \ldots \ldots \ldots \ldots \ldots \ldots \ldots \ldots \ldots \ldots . . . . .1-30-74$ & 26 \\
\hline 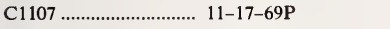 & 37 & $1171 \ldots \ldots \ldots \ldots \ldots \ldots \ldots \ldots \ldots \ldots . .7-71 P$ & 26 \\
\hline $1108 \ldots \ldots \ldots \ldots \ldots \ldots \ldots \ldots \ldots \ldots . . .11-17-69 \mathrm{P}$ & 37 & $1172 \ldots \ldots \ldots \ldots \ldots \ldots \ldots \ldots . .7-7-71 P$ & 26 \\
\hline $\mathrm{C} 1108 \ldots \ldots \ldots \ldots \ldots \ldots \ldots \ldots \ldots . .11-17-69 \mathrm{P}$ & 37 & 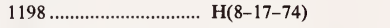 & 28 \\
\hline 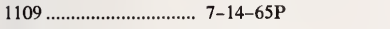 & 37 & 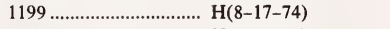 & 28 \\
\hline $\mathrm{C} 1109 \ldots \ldots \ldots \ldots \ldots \ldots \ldots \ldots \ldots \ldots . . .18-18-70 \mathrm{P}$ & 37 & 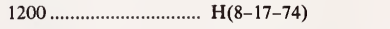 & 28 \\
\hline 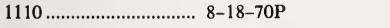 & 37 & 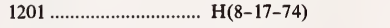 & 28 \\
\hline $\mathrm{C} 1110 \ldots \ldots \ldots \ldots \ldots \ldots \ldots \ldots \ldots \ldots . . .18-18-70 \mathrm{P}$ & 37 & 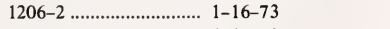 & 28 \\
\hline 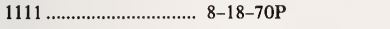 & 37 & 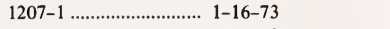 & 28 \\
\hline 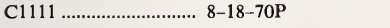 & 37 & 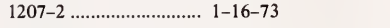 & 28 \\
\hline 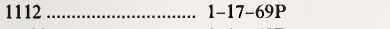 & 37 & 1208-1 ….................... 1-16-73 & 28 \\
\hline $\mathrm{C} 1112 \ldots \ldots \ldots \ldots \ldots \ldots \ldots \ldots \ldots . . .1-17-69 \mathrm{P}$ & 37 & 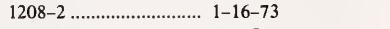 & 28 \\
\hline $1113 \ldots \ldots \ldots \ldots \ldots \ldots \ldots \ldots \ldots \ldots \ldots \ldots . . .1-17-69 P$ & 37 & 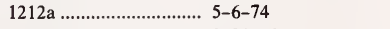 & 41 \\
\hline 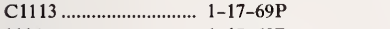 & 37 & 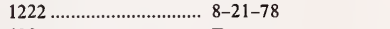 & 24 \\
\hline 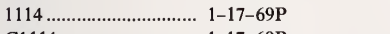 & 37 & 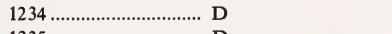 & 41 \\
\hline $\mathrm{C} 1114 \ldots \ldots \ldots \ldots \ldots \ldots \ldots \ldots \ldots \ldots . . .1-17-69 \mathrm{P}$ & 37 & 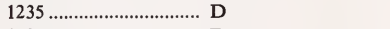 & 41 \\
\hline 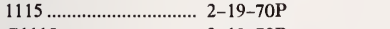 & 37 & 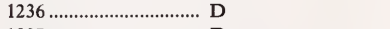 & 41 \\
\hline C1115 & 37 & 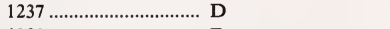 & 41 \\
\hline $1116 \ldots \ldots \ldots \ldots \ldots \ldots \ldots \ldots \ldots . . .2-19-70 P$ & 37 & 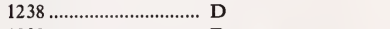 & 41 \\
\hline C1116 ........................ 2-19-70P & 37 & 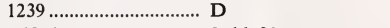 & 41 \\
\hline 1117 & 37 & C1251 ......................... 9-16-80 & 39 \\
\hline 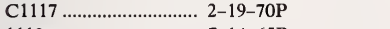 & 37 & 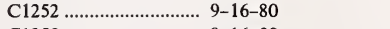 & 39 \\
\hline $1118 \ldots \ldots \ldots \ldots \ldots \ldots \ldots \ldots \ldots \ldots \ldots \ldots \ldots . . .14-65 P$ & 37 & 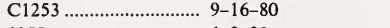 & 39 \\
\hline 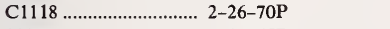 & 37 & 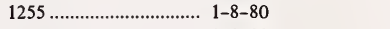 & 36 \\
\hline 1119 & 37 & 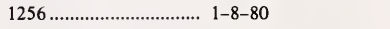 & 36 \\
\hline 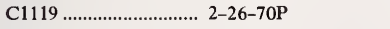 & 37 & 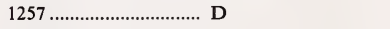 & 36 \\
\hline $\mathrm{C} 1120 \ldots \ldots \ldots \ldots \ldots \ldots . . .2-26-70 \mathrm{P}$ & 37 & 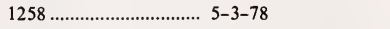 & 36 \\
\hline C1121 & 37 & 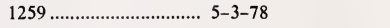 & 36 \\
\hline $1122 \ldots \ldots \ldots \ldots \ldots \ldots .12-19-66 \mathrm{P}$ & 37 & 1261a ........................ 2-24-81 & 24 \\
\hline $\mathrm{C} 1122 \ldots \ldots \ldots \ldots \ldots \ldots . .12-19-66 \mathrm{P}$ & 37 & 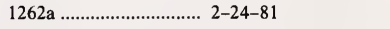 & 24 \\
\hline
\end{tabular}




\begin{tabular}{|c|c|c|c|}
\hline Certificate date & Page & Certificate date & Page \\
\hline $1263 a \ldots \ldots \ldots \ldots \ldots \ldots \ldots \ldots \ldots . . . . .2-24-81$ & 24 & $1521 \ldots \ldots \ldots \ldots \ldots \ldots \ldots \ldots \ldots \ldots \ldots$ & 73,83 \\
\hline $1264 a \ldots \ldots \ldots \ldots \ldots \ldots \ldots \ldots \ldots \ldots \ldots \ldots \ldots \ldots \ldots$ & 24 & $1522 \ldots \ldots \ldots \ldots \ldots \ldots \ldots \ldots \ldots \ldots \ldots \ldots \ldots \ldots \ldots \ldots \ldots$ & 73,83 \\
\hline 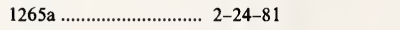 & 24 & 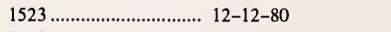 & 73 \\
\hline 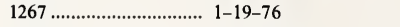 & 26 & 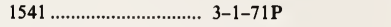 & 81 \\
\hline 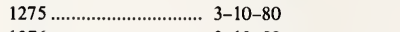 & 37 & 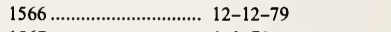 & 46 \\
\hline 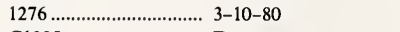 & 37 & 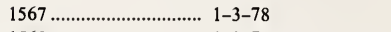 & 46 \\
\hline 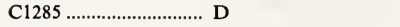 & ………….............. & 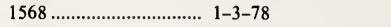 & 46 \\
\hline 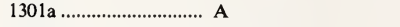 & 64 & 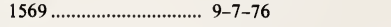 & 46 \\
\hline 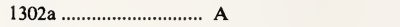 & 64 & 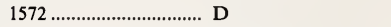 & 60 \\
\hline 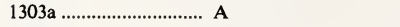 & 64 & 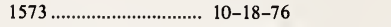 & 46 \\
\hline 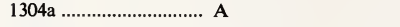 & 64 & $1575 \ldots \ldots \ldots \ldots \ldots+10-18-76$ & 46 \\
\hline $1305 \mathrm{a} \ldots \ldots \ldots \ldots \ldots \ldots \ldots \ldots \ldots \ldots \ldots$ & 64 & 1577a .......................... D & 60 \\
\hline $1306 \mathrm{a} \ldots \ldots \ldots \ldots \ldots \ldots \ldots \ldots \ldots \ldots \ldots$ & 64 & 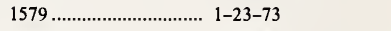 & 48 \\
\hline 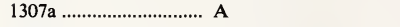 & 64 & 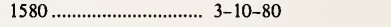 & 50 \\
\hline 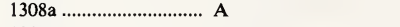 & 64 & 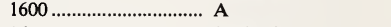 & 84 \\
\hline $1310 \mathrm{a} \ldots \ldots \ldots \ldots \ldots \ldots \ldots \ldots \ldots \ldots$ & 64 & 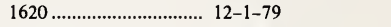 & 48 \\
\hline 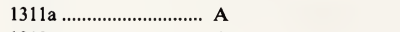 & 64 & 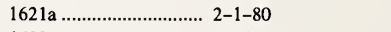 & 48 \\
\hline 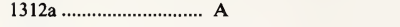 & 64 & $1622 a$ & 48 \\
\hline 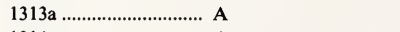 & 64 & 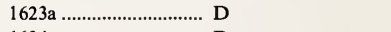 & 48 \\
\hline 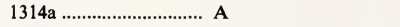 & 64 & 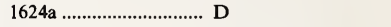 & 48 \\
\hline 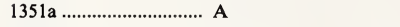 & 64 & $1625 \ldots \ldots \ldots \ldots \ldots \ldots \ldots \ldots$ & 51 \\
\hline $1352 \mathrm{a} \ldots \ldots \ldots \ldots \ldots \ldots \ldots \ldots \ldots \ldots \ldots$ & 65 & $1626 \ldots \ldots \ldots \ldots \ldots \ldots \ldots \ldots \ldots \ldots \ldots \ldots \ldots \ldots \ldots$ & 51 \\
\hline $1353 \mathrm{a} \ldots \ldots \ldots \ldots \ldots \ldots \ldots \ldots \ldots \ldots \ldots \ldots$ & 65 & 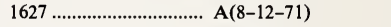 & 51 \\
\hline 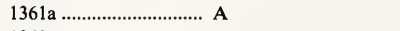 & 64 & 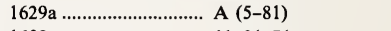 & 51 \\
\hline 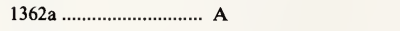 & 64 & 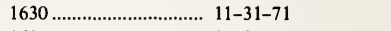 & 48 \\
\hline $1363 \mathrm{a} \ldots \ldots \ldots \ldots \ldots \ldots \ldots \ldots \ldots \ldots \ldots$ & 64 & 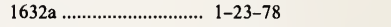 & 49 \\
\hline 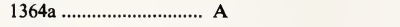 & 65 & $1633 a$ & 49 \\
\hline 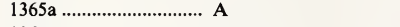 & 65 & $1634 \mathrm{a}$ & 49 \\
\hline 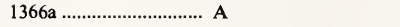 & 65 & 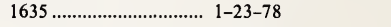 & 49 \\
\hline 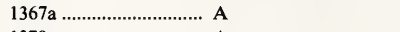 & 65 & 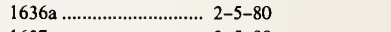 & 48 \\
\hline $1370 \mathrm{a} \ldots \ldots \ldots \ldots \ldots \ldots \ldots \ldots \ldots$ & 65 & 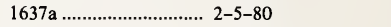 & 48 \\
\hline 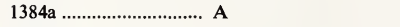 & 66 & 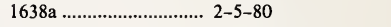 & 48 \\
\hline $1398 \mathrm{a} \ldots \ldots$ & 65 & 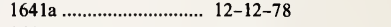 & 48 \\
\hline $1399 \mathrm{a} \ldots \ldots \ldots \ldots \ldots \ldots \ldots \ldots \ldots$ & 66 & 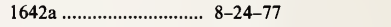 & 48 \\
\hline 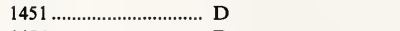 & 74 & 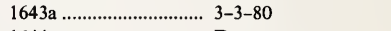 & 49 \\
\hline 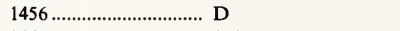 & 72 & 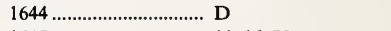 & 50 \\
\hline 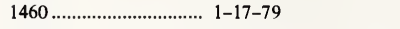 & 73,83 & 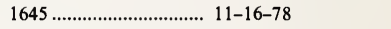 & 49 \\
\hline $1461 \ldots 17-79$ & 73,83 & 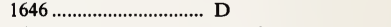 & 49 \\
\hline 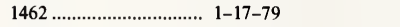 & 73,83 & 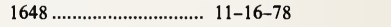 & 49 \\
\hline $1463 \ldots \ldots \ldots \ldots \ldots \ldots$ & 73,83 & 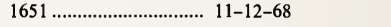 & 72 \\
\hline 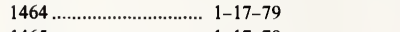 & 73,83 & 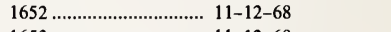 & 72 \\
\hline 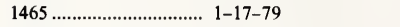 & 73,83 & 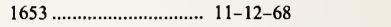 & 72 \\
\hline $1466 \ldots \ldots \ldots \ldots \ldots \ldots \ldots 1-17-79$ & 73,83 & 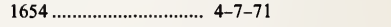 & 72 \\
\hline $1467 \ldots \ldots \ldots$ & 73,83 & 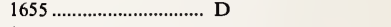 & 72 \\
\hline 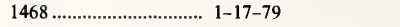 & 73,83 & $1658 \mathrm{a} \ldots \ldots \ldots \ldots \ldots \ldots \ldots \ldots \ldots \ldots$ & 47 \\
\hline 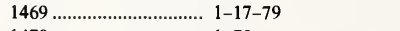 & 73,83 & 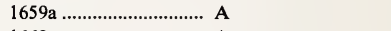 & 47 \\
\hline 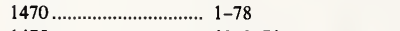 & 82 & $1660 \mathrm{a}$ & 47 \\
\hline 1475 & $69,70,72$ & $1661 \mathrm{a} \ldots \ldots \ldots$ & 47 \\
\hline 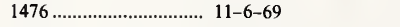 & 69,70 & $1662 a \ldots \ldots \ldots \ldots \ldots$ & 47 \\
\hline 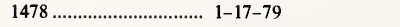 & 69,70 & $1663 a \ldots \ldots \ldots \ldots \ldots \ldots \ldots$ & 47 \\
\hline 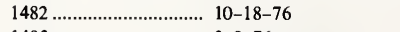 & 69,70 & 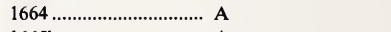 & 47 \\
\hline 1483 & 69,70 & $1665 \mathrm{~b}$ & 47 \\
\hline 1484 & 69,70 & $1666 \mathrm{~b}$ & 47 \\
\hline 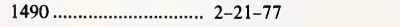 & 70 & $1667 \mathrm{~b} \ldots \ldots \ldots \ldots \ldots \ldots \ldots \ldots \ldots \ldots \ldots \ldots$ & 47 \\
\hline 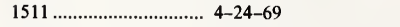 & 83,92 & $1668 \mathrm{~b} \ldots \ldots \ldots \ldots \ldots \ldots \ldots$ & 47 \\
\hline $1512 \ldots \ldots$ & 83,92 & 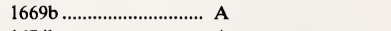 & 47 \\
\hline 1513 & 83,92 & 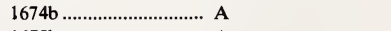 & 47 \\
\hline 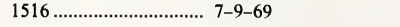 & 83,92 & $1675 \mathrm{~b} \ldots \ldots \ldots \ldots \ldots \ldots$ & 47 \\
\hline $1517 \ldots \ldots \ldots \ldots \ldots \ldots \ldots+1,7-9-69$ & 83,92 & $1677 \mathrm{c} \ldots \ldots \ldots \ldots \ldots$ & 47 \\
\hline 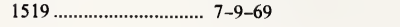 & 83,92 & $1678 \mathrm{c} \ldots \ldots \ldots \ldots \ldots \ldots \ldots \ldots \ldots \ldots$ & 47 \\
\hline
\end{tabular}




\begin{tabular}{|c|c|c|c|}
\hline Certificate date & Page & Certificate date & Page \\
\hline $1679 \mathrm{c}$ & 47 & $2620 \mathrm{a}$ & 47 \\
\hline $1680 \mathrm{~b}$ & 47 & $2621 \mathrm{a} \ldots \ldots \ldots \ldots \ldots \ldots . . . . . . . . \ldots$ & 47 \\
\hline 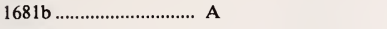 & 47 & $2622 \mathrm{a}$ & 47 \\
\hline 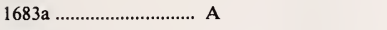 & 47 & 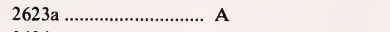 & 47 \\
\hline 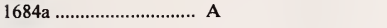 & 47 & $2624 a$ & 47 \\
\hline $1685 a$ & 47 & $2625 \mathrm{a} \ldots \ldots \ldots \ldots \ldots \ldots \ldots \ldots \ldots$ & 47 \\
\hline 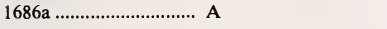 & 47 & $2626 a \ldots \ldots \ldots \ldots \ldots \ldots . . . . . . . \ldots$ & 47 \\
\hline 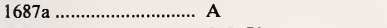 & 47 & 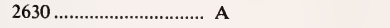 & 48 \\
\hline 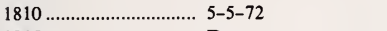 & 87 & 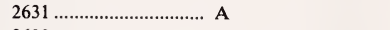 & 48 \\
\hline 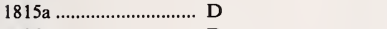 & 83 & $2632 \ldots \ldots \ldots \ldots \ldots \ldots \ldots \ldots$ & 48 \\
\hline 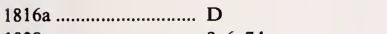 & 83 & $2633 \ldots \ldots \ldots \ldots \ldots \ldots \ldots \ldots$ & 48 \\
\hline 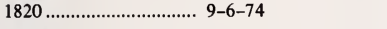 & 52,77 & 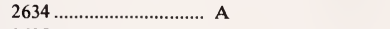 & 48 \\
\hline $1821 \mathrm{a} \ldots \ldots \ldots \ldots \ldots \ldots \ldots . . . \mathrm{D}$ & (n)........................ & 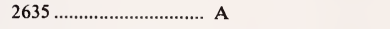 & 48 \\
\hline 1822 & 52,77 & 2637 & 48 \\
\hline $1823 \ldots . .12-9-76$ & 52,77 & 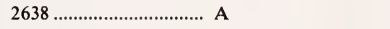 & 48 \\
\hline 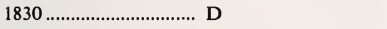 & 56 & 2639 & 48 \\
\hline 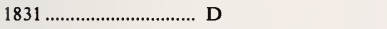 & 56 & 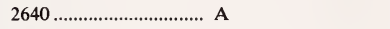 & 48 \\
\hline 1901 & 85 & 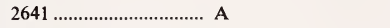 & 48 \\
\hline 1967 & 74 & 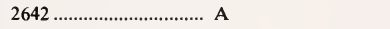 & 48 \\
\hline 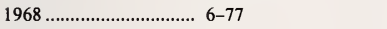 & 45,71 & 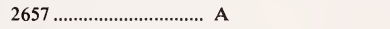 & 48 \\
\hline 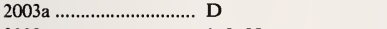 & 76 & $2658 \ldots \ldots \ldots \ldots \ldots \ldots \ldots \ldots \ldots \ldots \ldots \ldots \ldots \ldots \ldots \ldots$ & 48 \\
\hline $2009 \ldots \ldots \ldots \ldots \ldots \ldots \ldots \ldots \ldots \ldots \ldots \ldots . . .1-8-80$ & 76 & 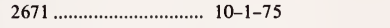 & 51 \\
\hline 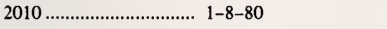 & 76 & 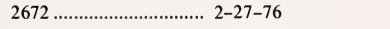 & 51 \\
\hline 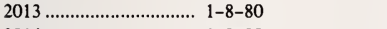 & 76 & 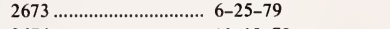 & 51 \\
\hline 2014 & 76 & $2674 \ldots \ldots \ldots \ldots \ldots \ldots \ldots \ldots \ldots \ldots \ldots \ldots \ldots . .12-18-79$ & 51 \\
\hline 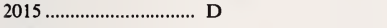 & 77 & $2675 \ldots \ldots \ldots \ldots \ldots \ldots \ldots \ldots \ldots \ldots \ldots \ldots \ldots . . .19-75$ & 51 \\
\hline 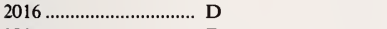 & 77 & 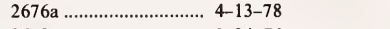 & 51 \\
\hline 2019 & 77 & $2679 \ldots \ldots \ldots \ldots \ldots \ldots \ldots \ldots . .9-24-76$ & 51 \\
\hline 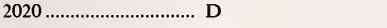 & 77 & 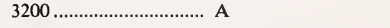 & 84 \\
\hline $2021 \ldots \ldots \ldots \ldots \ldots \ldots \ldots \ldots \ldots . . . . . . \ldots$ & 77 & 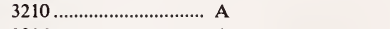 & 84 \\
\hline 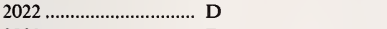 & 77 & 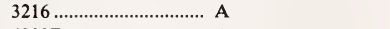 & 84 \\
\hline 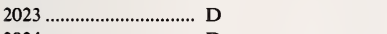 & 76 & 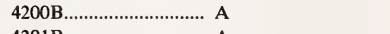 & 80 \\
\hline 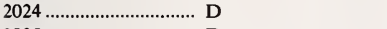 & 76 & 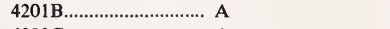 & 80 \\
\hline 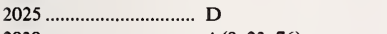 & 76 & 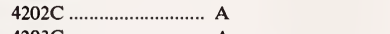 & 80 \\
\hline $2030 \ldots . . .4(9-23-76)$ & 76 & 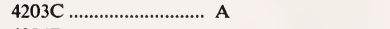 & 80 \\
\hline $2031 \ldots \ldots \ldots \ldots \ldots \ldots \ldots \ldots \ldots \ldots \ldots \ldots$ & 76 & 4206B......................... A & \\
\hline $2032 \ldots \ldots \ldots \ldots \ldots \ldots \ldots \ldots \ldots \ldots . .10-31-79$ & 76 & 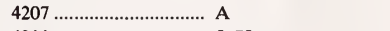 & 80 \\
\hline $2033 \ldots \ldots \ldots \ldots \ldots \ldots \ldots \ldots \ldots \ldots \ldots \ldots \ldots . . .5-90$ & 76 & $4211 \ldots \ldots \ldots \ldots \ldots \ldots \ldots \ldots \ldots \ldots \ldots . . .5-70$ & 80 \\
\hline 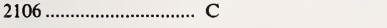 & 86 & 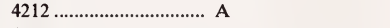 & 80 \\
\hline 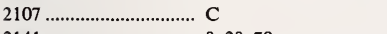 & 86 & 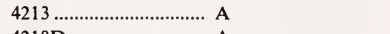 & 80 \\
\hline 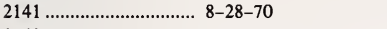 & 44 & $4218 \mathrm{D}$ & 80 \\
\hline $2142 \ldots \ldots \ldots \ldots \ldots \ldots \ldots \ldots \ldots \ldots \ldots \ldots . . .1-70$ & 44 & 4219B........................ 6-74 & 78 \\
\hline $2143 \ldots \ldots \ldots \ldots \ldots \ldots \ldots \ldots \ldots . . .1-23-73$ & 44 & 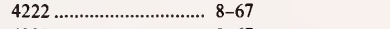 & 78 \\
\hline $2144 \ldots \ldots \ldots \ldots \ldots \ldots \ldots \ldots \ldots \ldots \ldots \ldots . . . .4-5-73$ & 44 & 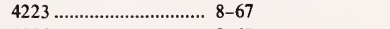 & 78 \\
\hline 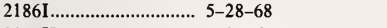 & 62 & 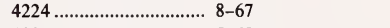 & 78 \\
\hline 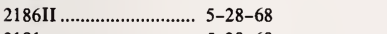 & 62 & 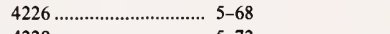 & 78 \\
\hline $2191 \ldots \ldots \ldots \ldots \ldots \ldots \ldots \ldots \ldots \ldots \ldots . . .5-28-68$ & 62 & $4229 \ldots \ldots \ldots \ldots \ldots \ldots \ldots \ldots \ldots \ldots \ldots \ldots \ldots . . .5-72$ & 78 \\
\hline 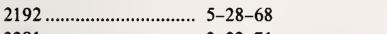 & 62 & 4233В & 78 \\
\hline 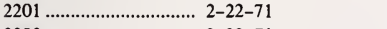 & 62 & 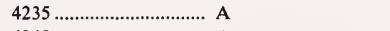 & 79 \\
\hline $2202 \ldots \ldots \ldots \ldots \ldots \ldots \ldots \ldots . . .2-22-71$ & 62 & 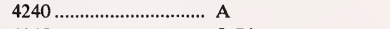 & 80 \\
\hline $2203 \ldots \ldots \ldots \ldots \ldots \ldots \ldots \ldots \ldots . .5-21-73$ & 62 & $4245 \ldots \ldots \ldots \ldots \ldots \ldots \ldots \ldots \ldots \ldots . . . .5-74$ & 78 \\
\hline 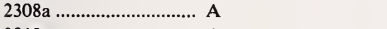 & 66 & 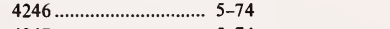 & 78 \\
\hline $2318 \mathrm{a} \ldots \ldots \ldots \ldots \ldots \ldots \ldots \ldots \ldots \ldots$ & 66 & 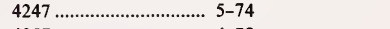 & 78 \\
\hline $2338 \mathrm{a} \ldots \ldots \ldots \ldots \ldots \ldots \ldots$ & 66 & 4257 & 78 \\
\hline 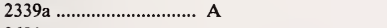 & 66 & 4260B.......................... A & 80 \\
\hline 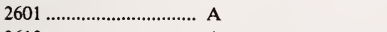 & 75 & $4302 \ldots \ldots \ldots \ldots \ldots \ldots$ & 79 \\
\hline $2612 \mathrm{a} \ldots \ldots \ldots \ldots \ldots \ldots \ldots \ldots \ldots \ldots$ & 47 & 4308 & 79 \\
\hline 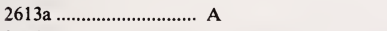 & 47 & 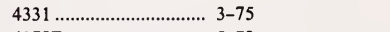 & 78 \\
\hline 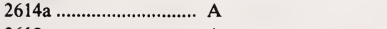 & 47 & 4370B........................... 5-78 & 78 \\
\hline $2619 a \ldots \ldots$ & 47 & 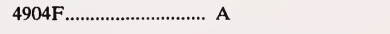 & 79 \\
\hline
\end{tabular}




\begin{tabular}{|c|c|c|c|c|}
\hline SRM & Certificate date & Page & Certificate date & Page \\
\hline 4906B.............. & $5-69$ & 79 & U-500 & 60 \\
\hline 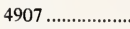 & A & 79 & U-750 & 60 \\
\hline 4919D ................. & $7-75$ & 78 & U-800 & 60 \\
\hline $4922 \mathrm{~L} \ldots \ldots \ldots \ldots$ & $\mathbf{A}$ & 78 & U-850 & 60 \\
\hline $4925 \ldots \ldots \ldots \ldots \ldots . . .$. & $7-58$ & 78 & U-900 & 60 \\
\hline $4926 \mathrm{C} \ldots \ldots \ldots \ldots$ & $9-78$ & 78 & U-930 & 60 \\
\hline 4927B.................. & $9-78$ & 78 & U-970 & 60 \\
\hline $4929 \mathrm{C} \ldots \ldots \ldots \ldots . . . . .$. & $4-70$ & 78 & RM $1 \mathrm{C} \ldots \ldots \ldots \ldots \ldots \ldots \ldots \ldots \ldots \ldots \ldots \ldots \ldots$ & 88 \\
\hline $4935 \mathrm{C} \ldots \ldots \ldots \ldots . . . . .$. & $3-74$ & 79 & RM 1R ......................... E & 88 \\
\hline $4940 \mathrm{~B} \ldots \ldots \ldots \ldots \ldots$ & $2-76$ & 78 & 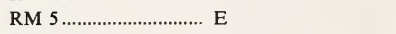 & 88 \\
\hline $4943 \ldots \ldots \ldots \ldots . . . . . .$. & 1962 & 78 & RM $31 \ldots \ldots \ldots \ldots \ldots \ldots \ldots \ldots \ldots$ & 88 \\
\hline 4947 & $9-78$ & 78 & RM $40 \ldots \ldots \ldots \ldots \ldots \ldots \ldots . . . . . . . \ldots$ & 88 \\
\hline $4950 \mathrm{D} \ldots \ldots \ldots \ldots . . . . .$. & A & 80 & RM 45b ........................ E & 88 \\
\hline $4951 \mathrm{C} \ldots \ldots \ldots \ldots . . . . .$. & . 4-78 & 80 & RM 49 & 79 \\
\hline $4952 \mathrm{~B} \ldots \ldots \ldots \ldots \ldots$ & $5-76$ & 80 & RM $50 \ldots \ldots \ldots \ldots \ldots \ldots \ldots \ldots \ldots \ldots \ldots \ldots$ & 88 \\
\hline $4953 \mathrm{C} \ldots \ldots \ldots \ldots . . . . .$. & A & 80 & GM $1 \ldots \ldots \ldots \ldots \ldots \ldots \ldots \ldots \ldots$ & 90 \\
\hline $4956 \ldots \ldots \ldots \ldots \ldots$ & $9-67$ & 81 & GM $2 \ldots \ldots \ldots \ldots \ldots \ldots \ldots \ldots \ldots . . F$ & 90 \\
\hline 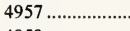 & A & 81 & GM $21 \ldots \ldots \ldots \ldots \ldots \ldots \ldots \ldots \ldots$ & 91 \\
\hline 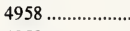 & A & 81 & 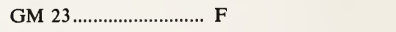 & 91 \\
\hline 4959 & $\mathbf{A}$ & 81 & GM $25 \ldots \ldots \ldots$ & 91 \\
\hline $4960 \ldots \ldots \ldots \ldots . . . .$. & A & 81 & GM $27 \ldots \ldots \ldots \ldots \ldots . . . F$ & 91 \\
\hline 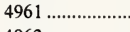 & A & 81 & GM $29 \ldots \ldots \ldots \ldots \ldots \ldots \ldots$ & 91 \\
\hline 4962 ...................... & A & 81 & GM $31 \ldots \ldots \ldots \ldots \ldots$ & 91 \\
\hline 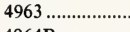 & A & $\begin{array}{l}81 \\
81\end{array}$ & 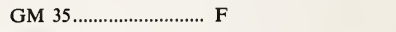 & 91 \\
\hline 4964B.................... & $\begin{array}{l}A \\
A(5-69)\end{array}$ & $\begin{array}{l}81 \\
80\end{array}$ & 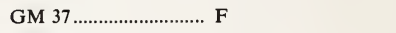 & 91 \\
\hline 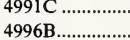 & $\begin{array}{l}A(5-69) \\
A(5-69)\end{array}$ & $\begin{array}{l}80 \\
80\end{array}$ & GM $39 \ldots \ldots \ldots \ldots \ldots \ldots \ldots$ & 91 \\
\hline 4997E & D & 80 & GM $40 \ldots \ldots \ldots \ldots \ldots \ldots \ldots$ & 91 \\
\hline 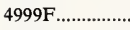 & $\mathrm{D}$ & 78 & 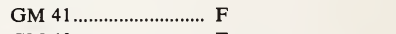 & 91 \\
\hline $6250 \ldots \ldots \ldots \ldots \ldots$ & A & 84 & GM $43 \ldots \ldots \ldots \ldots \ldots \ldots \ldots \ldots$ & 91 \\
\hline $\mathrm{U}-0002 \ldots \ldots \ldots \ldots$ & . 7-30-70P & 59 & GM $47 \ldots \ldots \ldots \ldots \ldots \ldots . . . F$ & 91 \\
\hline $\mathrm{U}-005 \ldots \ldots \ldots \ldots$ & 4-21-69P & 59 & GM $49 \ldots \ldots \ldots \ldots \ldots \ldots . . . F$ & 91 \\
\hline $\mathrm{U}-010 \ldots \ldots \ldots \ldots$ & 4-21-69P & 59 & GM $51 \ldots \ldots \ldots \ldots \ldots \ldots \ldots$ & 91 \\
\hline $\mathrm{U}-015 \ldots \ldots \ldots \ldots$ & 4-21-69P & 59 & GM $53 \ldots \ldots \ldots \ldots \ldots \ldots$ & 91 \\
\hline 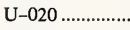 & $4-21-69 P$ & 59 & 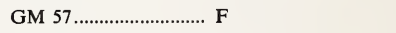 & 91 \\
\hline $\mathrm{U}-030 \ldots \ldots \ldots \ldots$ & 4-21-69P & 60 & 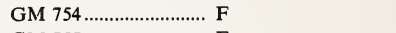 & 88 \\
\hline 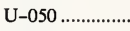 & $4-21-69 P$ & 60 & GM $757 \ldots \ldots \ldots \ldots \ldots . . . F$ & 88 \\
\hline U-100 ................. & $6-23-66 \mathrm{P}$ & 60 & 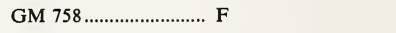 & 88 \\
\hline U-150 .................. & $8-5-66 \mathrm{P}$ & 60 & GM $759 \ldots \ldots \ldots \ldots \ldots \ldots$ & 88 \\
\hline U-200 ................. & $6-1-66 \mathrm{P}$ & 60 & GM $760 \ldots \ldots \ldots \ldots . . . F$ & 88 \\
\hline 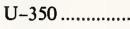 & ........... 5-23-66P & 60 & 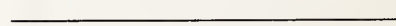 & \\
\hline
\end{tabular}










\section{NBS TECHNICAL PUBLICATIONS}

\section{PERIODICALS}

JOURNAL OF RESEARCH-The Journal of Research of the National Bureau of Standards reports NBS research and development in those disciplines of the physical and engineering sciences in which the Bureau is active. These include physics, chemistry, engineering, mathematics, and computer sciences. Papers cover a broad range of subjects, with major emphasis on measurement methodology and the basic technology underlying standardization. Also included from time to time are survey articles on topics closely related to the Bureau's technical and scientific programs. As a special service to subscribers each issue contains complete citations to all recent Bureau publications in both NBS and nonNBS media. Issued six times a year. Annual subscription: domestic $\$ 13$; foreign $\$ 16.25$. Single copy, \$3 domestic; $\$ 3.75$ foreign.

NOTE: The Journal was formerly published in two sections: Section A "Physics and Chemistry" and Section B "Mathematical Sciences."

DIMENSIONS/NBS-This monthly magazine is published to inform scientists, engineers, business and industry leaders, teachers, students, and consumers of the latest advances in science and technology, with primary emphasis on work at NBS. The magazine highlights and reviews such issues as energy research, fire protection, building technology, metric conversion, pollution abatement, health and safety, and consumer product performance. In addition, it reports the results of Bureau programs in measurement standards and techniques, properties of matter and materials, engineering standards and services, instrumentation, and automatic data processing. Annual subscription: domestic \$11; foreign $\$ 13.75$.

\section{NONPERIODICALS}

Monographs-Major contributions to the technical literature on various subjects related to the Bureau's scientific and technical activities.

Handbooks-Recommended codes of engineering and industrial practice (including safety codes) developed in cosperation with interested industries, professional organizations, and regulatory bodies.

Special Publications-Include proceedings of conferences sponsored by NBS, NBS annual reports, and other special publications appropriate to this grouping such as wall charts, pocket cards, and bibliographies.

Applied Mathematics Series-Mathematical tables, manuals, and studies of special interest to physicists, engineers, chemists, biologists, mathematicians, computer programmers, and others engaged in scientific and technical work.

National Standard Reference Data Series-Provides quantitative data on the physical and chemical properties of materials, compiled from the world's literature and critically evaluated. Developed under a worldwide program coordinated by NBS under the authority of the National Standard Data Act (Public Law 90-396).
NOTE: The principal publication outlet for the foregoing data is the Journal of Physical and Chemical Reference Data (JPCRD) published quarterly for NBS by the American Chemical Society (ACS) and the American Institute of Physics (AIP). Subscriptions, reprints, and supplements available from ACS, 1155 Sixteenth St., NW, Washington, DC 20056.

Building Science Series-Disseminates technical information developed at the Bureau on building materials, components, systems, and whole structures. The series presents research results, test methods, and performance criteria related to the structural and environmental functions and the durability and safety characteristics of building elements and systems.

Technical Notes-Studies or reports which are complete in themselves but restrictive in their treatment of a subject. Analogous to monographs but not so comprehensive in scope or definitive in treatment of the subject area. Often serve as a vehicle for final reports of work performed at NBS under the sponsorship of other government agencies

Voluntary Product Standards-Developed under procedures published by the Department of Commerce in Part 10, Title 15, of the Code of Federal Regulations. The standards establish nationally recognized requirements for products, and provide all concerned interests with a basis for common understanding of the characteristics of the products. NBS administers this program as a supplement to the activities of the private sector standardizing organizations.

Consumer Information Series-Practical information, based on NBS research and experience, covering areas of interest to the consumer. Easily understandable language and illustrations provide useful background knowledge for shopping in today's technological marketplace.

Order the above NBS publications from: Superintendent of Documents, Government Printing Office, Washington, DC 20402.

Order the following NBS publications-FIPS and NBSIR's-from the National Technical Information Services, Springfield, VA 22161.

Federal Information Processing Standards Publications (FIPS PUB)-Publications in this series collectively constitute the Federal Information Processing Standards Register. The Register serves as the official source of information in the Federal Government regarding standards issued by NBS pursuant to the Federal Property and Administrative Services Act of 1949 as amended, Public Law 89-306 (79 Stat. 1127), and as implemented by Executive Order 11717 (38 FR 12315, dated May 11, 1973) and Part 6 of Title 15 CFR (Code of Federal Regulations).

NBS Interagency Reports (NBSIR)-A special series of interim or final reports on work performed by NBS for outside sponsors (both government and non-government). In general, initial distribution is handled by the sponsor; public distribution is by the National Technical Information Services, Springfield, VA 22161, in paper copy or microfiche form. 
U.S. DEPARTMENT OF COMMERCE

National Bureau of Standards

Washington, DC 20234

DFFICIAL BUSINESS

Penalty for Private Use, $\$ 300$ 